Sebastian Hess

Meta-Analyse angewandter

Gleichgewichtsmodelle des internationalen

Agrarhandels 


\section{Meta-Analyse angewandter Gleichgewichtsmodelle des internationalen Agrarhandels}

Quantitative partielle- oder allgemeine Gleichgewichtsmodelle stellen in der angewandten Ökonomie ein wichtiges Instrument zur Politikfolgenabschätzung dar. Aus wissenschaftlicher Sicht wird an diesen Modellen ihre mangelnde Transparenz sowie ihre Abhängigkeit von empirisch wenig gesicherten Parametern und Annahmen kritisiert. Mittels einer Meta-Analyse werden daher Studien untersucht, welche Politikfolgen der laufenden WTO-Verhandlungen (Doha-Runde) simuliert haben. Als zentrales Ergebnis zeigt sich, dass die erhebliche Varianz innerhalb simulierter regionaler Einkommensveränderungen durch vergleichsweise wenige, ermittelbare Variablen zu einem hohen Anteil erklärt werden kann. Die Ergebnisse der Meta-Regression können daher als Orientierungshilfe bei Vergleich und Bewertung existierender sowie zukünftiger Simulationsstudien dienen.

Sebastian Hess, geboren 1975 in Kassel; 1998-2000 Studium an der Universität Göttingen; 2000-2001 Studium an der University of California, Davis (EAPScholarship); 2003 Examen M.Sc. Agricultural Economics; Promotionsstipendium der Friedrich-Naumann-Stiftung; zurzeit Wissenschaftlicher Mitarbeiter am Department für Agrarökonomie und Rurale Entwicklung der Universität Göttingen; 2007 Promotion. 
Meta-Analyse angewandter Gleichgewichtsmodelle des internationalen Agrarhandels 


\title{
cege-Schriften
}

Centrum für Europa-, Governance- und Entwicklungsforschung (cege)

Georg-August-Universität Göttingen

Center for Europeon, Governance and Economic Development Research (cege)

\section{Band 15}

\author{
Herausgegeben von \\ Kilian Bizer, Stephan von Cramon-Taubadel, \\ Andreas Oestreicher, Renate Ohr, \\ Gerhard Rübel, Robert Schwoger, \\ Verantwortlicher Herausgeber für diesen Band: \\ Stephan von Cramon-Taubadel
}

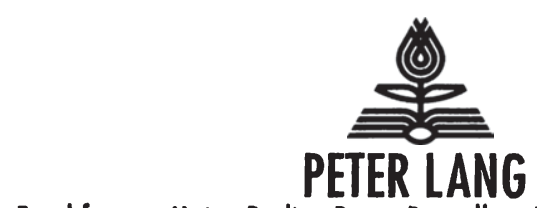

Frankfurt am Main - Berlin - Bern - Bruxelles - New York - Oxford - Wien 


\section{Sebastian Hess}

\section{Meta-Analyse angewandter Gleichgewichtsmodelle des internationalen Agrarhandels}

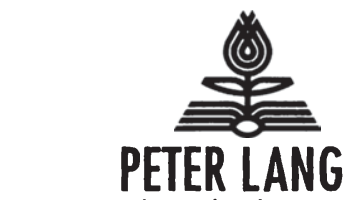

Internationaler Verlog der Wissenschaften 


\title{
Bibliografische Information der Deutschen Nationalbibliothek
}

Die Deutsche Nationalbibliothek verzeichnet diese Publikation in der Deutschen Nationalbibliografie; detaillierte bibliografische Daten sind im Internet über <http://www.d-nb.de> abrufbar.

Open Access: The online version of this publication is published on www.peterlang.com and www.econstor.eu under the international Creative Commons License CC-BY 4.0. Learn more on how you can use and share this work: http://creativecommons. org/licenses/by/4.0.

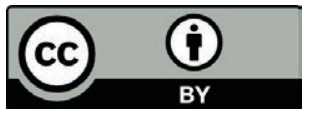

This book is available Open Access thanks to the kind support of ZBW - Leibniz-Informationszentrum Wirtschaft.

Zugl.: Göttingen, Univ., Diss., 2007

Gedruckt auf alterungsbeständigem, säurefreiem Papier.

\author{
D 7
}

ISSN1617-741X

ISBN 978-3-631-57718-9

ISBN 978-3-631-75694-2 (eBook)

(c) Peter Lang GmbH

Internationaler Verlag der Wissenschaften

Frankfurt am Main 2008

Alle Rechte vorbehalten.

Das Werk einschließlich aller seiner Teile ist urheberrechtlich geschützt. Jede Verwertung außerhalb der engen Grenzen des

Urheberrechtsgesetzes ist ohne Zustimmung des Verlages unzulässig und strafbar. Das gilt insbesondere für Vervielfältigungen, Übersetzungen, Mikroverfilmungen und die Einspeicherung und Verarbeitung in elektronischen Systemen.

Printed in Germany 123457

www.peterlang.de 


\section{Vorwort}

Quantitative, berechenbare ökonomische Modelle bilden partielle- oder allgemeine Marktgleichgewichte ab und stellen in der angewandten Ökonomie ein wichtiges und häufig eingesetztes Instrument zur Politikfolgenabschätzung und zur Politikberatung dar. Beispielsweise werden Modellsimulationen häufig zur Beantwortung von Fragen im Zusammenhang mit der laufenden Doha-Runde verwendet. Hierbei geht es etwa um die Frage, ob Entwicklungsländer durch einen erfolgreichen Abschluss der Runde gewinnen oder verlieren würden. Aus wissenschaftlicher Sicht wird an diesen Modellen aber ihre mangelnde Transparenz sowie ihre Abhängigkeit von theoretisch mitunter wenig plausiblen Annahmen kritisiert.

In der vorliegenden Arbeit verwendet Dr. Sebastian Hess meta-analytische Methoden, um die Transparenz von gleichgewichtsmodellbasierten Simulationsergebnissen zu verbessern. Letztlich geht es um die Fragen warum Modelle mitunter so unterschiedliche Simulationsergebnisse für anscheinend gleiche Szenarien (z.B. vollständige Agrarliberalisierung) produzieren, und ob diese Unterschiede von wichtigen Modellkomponenten (z.B. Annahmen über Skalenerträge, das Aggregationsniveau, die verwendete Datenbank, und viele mehr) abhängen. Durch die systematische, quantitative Auswertung von Simulationsergebnissen aus einer repräsentativen Stichprobe von veröffentlichten modelbasierten Simulationsstudien gelingt es Herrn Hess, die Grenzen von einfachen, meist auf wenige Parameter beschränkten Sensitivitätsanalysen einerseits, und subjektiven, partiellen Literaturvergleichen andererseits zu überwinden.

Die in der Arbeit von Herrn Hess dokumentierten Ergebnisse können wie folgt zusammengefasst werden. Erstens, der Vergleich von Simulationsexperimenten erfordert zunächst eine Definition der zu betrachtenden Literaturgrundgesamtheit. Meta-Analyse erhebt den Anspruch, einen bestimmten Bereich der Literatur repråsentativ abzubilden. Hierzu hat Herr Hess eine Verfahrensweise entwickelt, welche der heterogenen Schlagwortstruktur im Bereich eines angewandten ökonomischen Themas Rechnung trägt.

Zweitens, obwohl für die meisten Simulationsmodelle inzwischen ausführliche Handbücher frei verfügbar sind, werden einzelne Szenarien innerhalb einer Publikation nur selten ausfuhrlich dokumentiert und sind anhand der veröffentlichten Beschreibung des experimentellen Aufbaus so gut wie nie exakt reproduzierbar. 
Drittens, als zentrales Ergebnis zeigt Herr Hess, dass die Varianz der simulierten Wohlfahrtseffekte innerhalb des gewonnen Meta-Datensatzes bedeutend ist, dass diese sich aber mittels einer ökonometrischen Meta-Analyse durch vergleichsweise wenige, ermittelbare Variablen zu einem hohen Anteil erklären lässt. Unter anderen zeigt sich, dass die sog. Armington-Annahme sowie Annahmen, die über Skalenerträge, Kapitalakkumulation sowie den technischen Fortschritt in einem Modell getroffen werden, besonders hohe Auswirkungen auf Simulationsergebnisse ausüben. Ferner hat die in einem Modelllauf verwendete Datenbasis (einige neuere Datenbasen enthalten z.B. Informationen über angewandte statt gebundene Zölle) wichtige Folgen für die Höhe der simulierten Wohlfahrtseffekte.

Als Resultat der Arbeit von Herrn Hess, die im Rahmen eines Forschungsprojektes der Deutschen Forschungsgemeinschaft gefördert und von der Fakultät für Agrarwissenschaften der Georg-August-Universität als Dissertation angenommen wurde, konnten zentrale Erkenntnisse gewonnen werden, die den eingeschlagenen methodischen Weg als viel versprechende Pionierarbeit ausweisen. Mit der in diesem Band dokumentierten Forschung ist es Herrn Dr. Hess gelungen, einen wichtigen Beitrag zur Transparenz von quantitativen Simulationsmodellen in der Ökonomie zu leisten. Er erstellt Benchmarks, die Modellierer zukünftig werden berücksichtigen müssen, und die auch zur Glaubwürdigkeit der modellgestützten ökonomischen Politikberatung beitragen können.

Göttingen, im Januar 2008

Prof. Dr. Stephan von Cramon-Taubadel 


\section{Danksagung}

Die vorliegende Dissertation ist das Ergebnis meiner Zeit als Stipendiat sowie meiner Arbeit als wissenschaftlicher Mitarbeiter am Department für Agrarökonomie und Rurale Entwicklung. Vom 1. April 2004 bis 30. September 2006 erhielt ich ein Promotionsstipendium der Friedrich-Naumann-Stiftung sowie einen Reisekostenzuschuss für den Forschungsaufenthalt in Purdue, USA .

Die Deutsche Forschungsgemeinschaft DFG hat das Projekt „Evaluation angewandter Gleichgewichtsmodelle des Agrarsektors: Quantifikation systematischer Zusammenhänge zwischen Modellspezifikationen und Prognoseergebnissen" als Neu- sowie als Fortsetzungsantrag gefördert.

Besonderer Dank gilt meinem Doktorvater Professor Dr. Stephan von Cramon-Taubadel. Er schaffte durch seinen verständnisvollen und fairen Führungsstil, seine fachliche Kompetenz sowie durch die Vermittlung höchster wissen-schaftlicher Ansprüche ein Umfeld, in dem freies Denken und Arbeiten gefördert und eigene Impulse durch konstruktive Hinweise zu neuen Zielen gelenkt wurden!

Ebenso danke ich Professor Dr. Bernhard Brümmer, der mit Professor Dr. Stephan von Cramon-Taubadel das DFG-Projekt leitete, für seinen fachlichen und moralischen Beistand und seine hilfreiche Kritik!

Frau Professor Dr. Martina Brockmeier ermöglichte mir die Diskussion mit Modellexperten der Bundesforschungsanstalt für Landwirtschaft in Braunschweig und hat dankenswerterweise die Zweitbegutachtung übernommen! Ferner danke ich insbesondere Professor Dr. Yves Surry (Uppsala) für wegweisende Ideen sowie für wichtige Hinweise auf relevante Literatur zu MetaAnalysen und angewandten Gleichgewichtsmodellen!

Dieses Projekt hat darüber hinaus maßgeblich von anonymen Gutachtern der DFG, des Journals The World Economy, der EAAE-Konferenz in Kopenhagen, der GeWiSoLa-Konferenz 2005 sowie von den Anregungen im Rahmen der Invited Panel Session während der IAAE-Konferenz in Gold Coast, Australien profitiert. Ich möchte diesen Gutachtern ausdrücklich und sehr herzlich danken!

Die Durchführung des Projektes wäre nicht möglich gewesen, ohne dass viele zeitweilig im Projekt beschäftigte Mitarbeiter ihr Fachwissen, ihre Kreativität und ihren Arbeitseinsatz eingebracht hätten und gemeinsam mit mir bereit gewesen wären, sich den vielen und immer wieder neuen Herausforderungen zu stellen. Ich hoffe, dass die Arbeit ihnen wie mir Spaß gemacht 
hat und viele Einsichten bescheren konnte. Ich danke Euch, Inken, Tinoush, Carsten, Markus, Dirk, Eberhard, Emanuel, Henning, Lorenz und Rico, für die großartige Zusammenarbeit!

Darüber hinaus gab es Menschen, die durch meist kurze, mitunter kritische, für mich aber gerade deshalb häufig wegweisende Kommentare ihr Interesse an diesem Projekt bekundeten und in wichtiger Weise - mutatis mutandis - zum Entstehen dieser Arbeit beigetragen haben. Ich möchte daher insbesondere Holger Bergmann, Martin Banse, Frank von Tongeren, Jesper Kuehl sowie meiner Schwester Cordelia und meinem Onkel Heinrich danken!

Aber es gab auch diejenigen, die in diese Arbeit nicht direkt eingebunden waren, die meine Abwesenheit und Anspannung über Jahre hingenommen und mir dennoch Ihre Geduld, Ihr Verständnis, ihren Rat und ihre Bedenken entgegengebracht haben. Insbesondere Constanze sowie meiner Mutter, meinen Freunden Basil, Bernhard, Henryk, Maike und Paul verdanke ich, dass mich Ausdauer und Zuversicht nie verlassen haben. Danke!

Göttingen, den 26. 9. 2007

Sebastian Hess 


\section{Inhaltsverzeichnis}

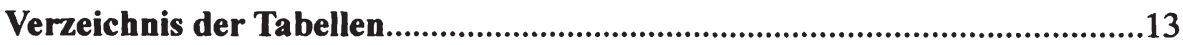

Verzeichnis der Abbildungen......................................................................15

Verzeichnis der Abkürzungen....................................................................17

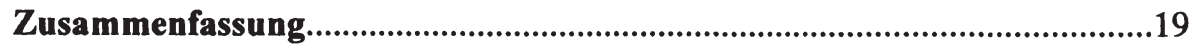

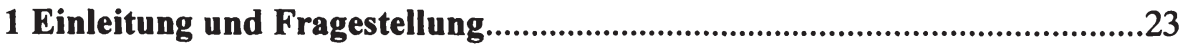

2 Evaluation angewandter Gleichgewichtsmodelle: Stand der Forschung. 29

2.1 Untersuchungsgegenstand: Was sind angewandte Gleichgewichts-

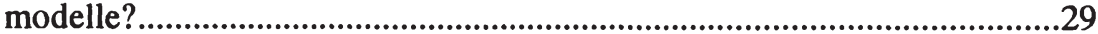

2.2 Stärken angewandter Gleichgewichtsmodelle........................................35

2.3 Kritik an angewandten Gleichgewichtsmodellen........................................37

2.3.1 Was determiniert Simulationsergebnisse innerhalb der Black

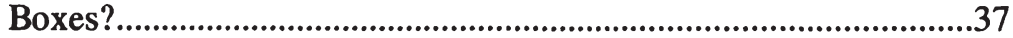

2.3.2 Unzureichende empirische Absicherung der Modelle......................41

2.3.3 Sind Simulationsergebnisse unterschiedlicher Black Boxes vergleichbar?

2.4 Theorie und Wirklichkeit: Die problematische Interpretation von

Simulationsergebnissen..................................................................................46

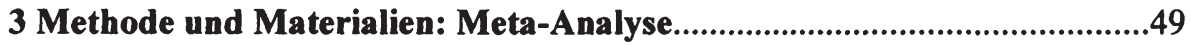

3.1 Meta-Analyse als quantitativer Ergebnisvergleich...................................49

3.2 Ökonometrische Aspekte der Meta-Analyse.........................................52

3.2.1 Meta-Analyse und lineare Regressionsmodelle.............................53

3.2.2 Multikollinearität ......................................................................55

3.2.3 Ansätze zur Varianzreduktion innerhalb der erklärenden Variab-

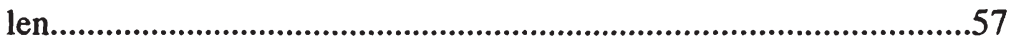

3.2.4 Heteroskedastizität...............................................................60

3.2.5 Serielle Korrelation/ Autokorrelation..........................................63

3.2.6 Fixed-, Random- oder Mixed-Effects............................................64

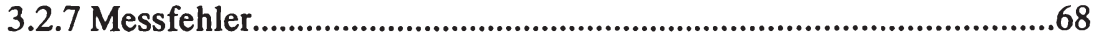

3.2.8 Die Annahme der Linearität......................................................69 
3.2.9 Modellselektion und Vorhersage.................................................69

3.3 Das Literatursample...................................................................................71

3.3.1 Die Literatur-Grundgesamtheit: Simulationen zur Doha-Runde.....72

3.3.2 Generierung einer repräsentativen Literaturauswahl.....................75

3.4 Vorstudien zur Meta-Analyse von Agrarsektormodellen...........................79

3.4.1 Zusammenfassung Hess (2005a)................................................80

3.4.2 Zusammenfassung Hess (2005b) und Hess (2006)..........................81

3.4.3 Zusammenfassung Hess und von Cramon-Taubadel

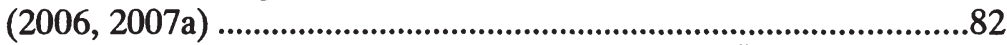

3.4.4 Erhebung und Approximation der Meta-Daten im Überblick..........84

3.5 Meta-Analyse: Definition der Variablen................................................85

3.5.1 Definition der zu erklärenden Variable:.........................................86

3.5.2 Definition der unabhängigen Variablen...........................................88

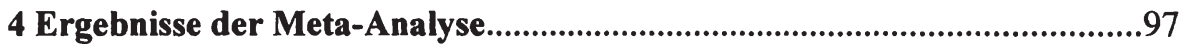

4.1 Deskriptive Auswertung des Meta-Datensatzes........................................97

4.2 Meta-Regression simulierter regionaler Wohlfahrtsänderungen..............109

4.3 Interpretation der Regressionskoeffizienten............................................110

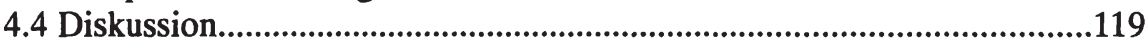

5 Erweiterung: Meta-Analyse und Response-Surface Design....................123

5.1 Response-Surface und Meta-Modellierung.............................................123

5.2 Applikation 1: Ein partiell-allgemeines Meta-Response-Surface............125

5.2.1 Experimentelles Design...........................................................127

5.2.2 Parametrische und Nicht-Parametrische Meta-Modellierung........130

5.3 Applikation 2: Meta-Modellierung bilateraler Zollpräferenzen im

Welt-Zuckermarkt.................................................................................134

6 Weiterer Forschungsbedarf..............................................................137

6.1 Mögliche Weiterentwicklungen im Bereich der Erhebung und

Standardisierung der Meta-Daten:........................................................137

6.2 Weiterentwicklung des ökonometrischen Meta-Modells: ........................138

6.3 Forschungsbedarf im Bereich der Response-Surface-Modellierung:......138

7 Fazit.

Literaturverzeichnis.

Anhang A: Das Literatursample. 
Anhang C: Ein Simulationsexperiment mit GTAP 189 
Sebastian Hess - 978-3-631-75694-2

Downloaded from PubFactory at 01/11/2019 02:52:17AM

via free access 


\section{Verzeichnis der Tabellen}

Tabelle 1: Beschreibung der Modellierungskonzepte und Studien in dem Meta-Datensatz.

Tabelle 2: Deskriptive Statistik der verwendeten Variablen in den Regression für aggregierte Weltwohlfahrtsgewinne.

Tabelle 3: OLS-Regression über simulierte Weltwohlfahrtsänderungen (ungewichtet, robuste Standardfehler).

Tabelle 4: OLS Regression über simulierte Weltwohlfahrtsänderungen (gewichtet, robuste Standardfehler).

Tabelle 5: Deskriptive Statistik der Variablen innerhalb der Meta-Regression für simulierte regionale Wohlfahrtsänderungen.

Tabelle 6: OLS-Regressionsergebnisse (robuste Standardfehler) der MetaRegression (siehe Erläuterung im Text).

Tabelle 7: OLS-Regressionsergebnisse (robuste Standardfehler, ungewichtet) der Meta-Regression; Dummies für jede aggregierte Region....117

Tabelle 8: OLS-Regressionsergebnisse (robuste Standardfehler, gewichtet) der Meta-Regression; Dummies für jede aggregierte Region.

Tabelle 9: Versuchsaufbau für ein partiell-allgemeines Meta-Modell.

Tabelle 10: Zwei Meta-SUR für Wohlfahrtsänderung und ToT. Ein vertikaler Vergleich der Koeffizienten innerhalb der Tabelle zeigt Unterschiede zwi- schen PE und CGE; ein horizontaler Vergleich zeigt die Unterschiede zwi- schen „Harberger" Dreiecken und ToT-Effekten des jeweiligen Modells.

Tabelle 11: PE-CGE-OLS-Meta-Modell mit robusten Standardfehlern. Fehlende Werte in GDP und $\eta D$ wurden durch Mittelwert dieser Variablen ersetzt.

Tabelle 12: Verwendete Literaturdatenbanken. 
Tabelle 13: Matrix der verwendeten Suchworte. 159

Tabelle 14: Koeffizienten regionaler Aggregationen (ungewichtet)................175

Tabelle 15: Koeffizienten regionaler Aggregationen (gewichtet).....................182 


\section{Verzeichnis der Abbildungen}

Abbildung 1: Simulierte Weltwohlfahrtseffekte gemäß einer Literaturzusammenstellung von UNCTAD (2003).

Abbildung 2: Simulierte Weltwohlfahrtseffekte eines repräsentativen Literatursamples. Die schwarz hinterlegten Säulen identifizieren diejenigen Studien, welche auch in Abbildung 1 vorkommen.

Abbildung 3: Das Literatursample. ................................................................77

Abbildung 4: Das System der Datenerhebung im Überblick.............................85

Abbildung 5: Scatterplot regionaler Wohlfahrtsveränderungen je Simulationsexperiment.

Abbildung 6: Armington-Elastizităten und weitere Modellspezifikationen im Vergleich. Erläuterung im Text.

Abbildung 7: Predicted Values der Weltwohlfahrtsregression Subsample „ohne B+L“, gewichtet....................................................................104

Abbildung 8: Simulierte Wohlfahrtseffekte für CGE (links) und PE (rechts)...128 
Sebastian Hess - 978-3-631-75694-2

Downloaded from PubFactory at 01/11/2019 02:52:17AM

via free access 


\section{Verzeichnis der Abkürzungen}

\begin{tabular}{|c|c|}
\hline ABARE & $\begin{array}{l}\text { Australian Bureau of Agricultural and Resource } \\
\text { Economics }\end{array}$ \\
\hline AIC & Akaike's Information Criterion \\
\hline ANOVA & Analysis of Variance \\
\hline a.v. & ad valorem \\
\hline $\mathrm{BIC}$ & Bayesian Information Criterion von Schwarz \\
\hline BLUE & Best Linear Unbiased Estimator \\
\hline BDS & $\begin{array}{l}\text { Brown, Deardorff und Stern Modell; Michigan } \\
\text { Model of Production and Trade }\end{array}$ \\
\hline BSP / BIP & Bruttosozialprodukt / Bruttoinlandsprodukt \\
\hline $\mathrm{B}+\mathrm{L}$ & $\begin{array}{l}\text { Studien innerhalb des Literatursamples mit den } \\
\text { Autoren Brown/Deardorff/Stern und } \\
\text { Lodefalk/Kinnman }\end{array}$ \\
\hline CAP & $\begin{array}{l}\text { Common Agricultural Policy der Europäischen } \\
\text { Union }\end{array}$ \\
\hline CGE & Computable General Equilibrium Model \\
\hline c.p. & ceteris paribus, „wobei die anderen gleich sind“" \\
\hline CES & Constant Elasticity of Substitution \\
\hline CRTS & Constant Returns to Scale, konstante Skalenerträge \\
\hline $\mathrm{CV}$ & Compensating Variation, kompensierende Variation \\
\hline EV & Equivalent Variation, äquivalente Variation \\
\hline FAO & $\begin{array}{l}\text { Food and Agricultural Organization of the United } \\
\text { Nations }\end{array}$ \\
\hline FAPRI & $\begin{array}{l}\text { Food and Agricultural Policy Research Institute } \\
\text { (Iowa State University) }\end{array}$ \\
\hline FDI & Foreign Direct Investment \\
\hline GATT & $\begin{array}{l}\text { General Agreement on Tariffs and Trade. Vorläufer } \\
\text { der World Trade Organization }\end{array}$ \\
\hline GE & General Equilibrium \\
\hline GLS & Generalized Least Squares \\
\hline GDP & Gross Domestic Product \\
\hline GTAP & Global Trade Analysis Project \\
\hline HS6 & Harmonized System Level 6 \\
\hline
\end{tabular}


IAAE

IGLS

IIT

IRTS

I-O

LP

MFN

ML

MLM

MSE

MTRI

NAFTA

NTB

OECD

OLS

PCA

PE

SAM

SQL

SUR

SSE

SST

TRI

ToT

UNCTAD

VBA

VIF

WLS

WTO
International Association of Agricultural

Economists

Iterative Generalized Least Square

Intra Industry Trade

Increasing Returns to Scale

Input-Output

Lineare Programmierung

Most Favoured Nation Tariff

Maximum Likelihood

Multilevel Model

Mean Squared Error

Mercantilist Trade Restrictiveness Index

North American Free Trade Agreement,

Mitgliedsländer USA, Kanada, Mexiko

Non Tariff Barriers, nicht-tarifäre

Handelshemmnisse

Organization for Economic Cooperation and

Development

Ordinary Least Squares, Kleinstquadratschätzer

Principal Component Analysis

Partial Equilibrium, partielles

Gleichgewichtsmodell

Social Account Matrice

Structured Query Language

Seemingly Unrelated Regression

Sum of Squared Errors

Sum of Squared Totals

Trade Restrictiveness Index

Terms-of-Trade

United Nations Conference on Trade and

Development

Visual Basic for Applications (Microsoft)

Varianzinflationsfaktor

Weighted Least Square

World Trade Organization 


\section{Zusammenfassung}

Während der laufenden WTO-Doha-Runde haben sich komplexe computergestützte Simulationsmodelle als verbreitete wissenschaftliche Methode etabliert, um die zu erwartenden Gewinne einer vollständigen oder teilweisen Abschaffung von Marktzugangsbarrieren zu errechnen. Die meisten dieser Marktzugangsbarrieren sind Zölle und werden in den für Entwicklungsländer besonders wichtigen Weltagrarmärkten erhoben. Simulationsrechnungen werden dabei als wichtige Grundlage für politische Verhandlungen z.T. stark instrumentalisiert, wobei bisher keine wissenschaftliche Methode existiert, um die Ergebnisse unterschiedlicher Simulationsmodelle miteinander vergleichen, geschweige denn sie quantitativ evaluieren zu können. Die gegenwärtig üblichen qualitativen Literaturvergleiche bleiben meist Erklärungen für divergierende Simulationsergebnisse $\mathrm{zu}$ anscheinend ähnlichen Fragestellungen schuldig, was bei politischen Entscheidungsträgern das allgemeine Vertrauen in simulierte Ergebnisse als eine objektive Entscheidungsgrundlage herabsetzt.

Die zum Einsatz kommenden Simulationsmodelle sind quantitative, berechenbare, angewandte, ökonomische Modelle. Sie bilden partielle oder allgemeine Marktgleichgewichte ab und stellen in der angewandten Ökonomie ein wichtiges und häufig eingesetztes Instrument zur Politikfolgenabschätzung dar. Aus wissenschaftlicher Sicht wird an diesen Modellen ihre mangelnde Transparenz (Black-Box-Charakter) sowie ihre Abhängigkeit von theoretisch mitunter wenig plausiblen Annahmen und empirisch kaum validierten Parametern kritisiert. In der vorliegenden Arbeit wurde daher auf Basis des methodischen Konzepts der Meta-Analyse eine ökonometrische Methode zur quantitativen Modellevaluation entwickelt, um Antworten auf die folgenden Forschungsfragen zu finden:

Kann das Zustandekommen von Simulationsergebnissen zu Fragen der Wohlfahrtswirkungen multiregionaler Handelsliberalisierung systematisch erklärt und über Studien und Modelle hinweg miteinander verglichen werden? Welche Aussagen können anhand bisher existierender Studien über durchschnittlich zu erwartende, simulierte Einkommens- (bzw. Wohlfahrts-) veränderungen in a) der Welt als geschlossener Volkswirtschaft und b) innerhalb einzelner Länder als Folge von Handelsliberalisierung getroffen werden? Unter welchen Bedingungen werden diese Veränderungen in der Wirklichkeit eintreten? Durch welche Voraussetzungen könnte die allgemeine Akzeptanz an- 
gewandter Gleichgewichtsmodelle und ihrer Ergebnisse als wissenschaftliche Methode zur quantitativen Politikfolgenabschätzung verbessert werden?

Hierzu wurde ein repräsentatives Literatursample mit 230 simulationsmodellbasierten Publikationen während der WTO-Doha-Runde erstellt. Es hat sich aber gezeigt, dass für die meisten Simulationsmodelle inzwischen zwar ausführliche Handbücher frei verfügbar sind, einzelne Simulationsszenarien innerhalb einer Publikation aber kaum ausfuhrlich dokumentiert werden und anhand der Beschreibung des experimentellen Aufbaus innerhalb einer Publikation so gut wie nie exakt reproduzierbar sind. Ein Internetsurvey mit den Autoren dieser Studien konnte diesbezüglich nur eingeschränkt eine bessere Datengrundlage ermöglichen, da die Autoren nicht in allen Fällen auch selbst die Simulationen durchgeführt, sondern hierzu mitunter auf namentlich nicht erwähnte Programmierer zurückgegriffen haben. Vor dem Hintergrund dieser Restriktionen konnten 110 der 230 ausgewählten Studien in die eigentliche Meta-Analyse aufgenommen werden.

Ferner wurde eine Referenzdatenbank erstellt, welche die Höhe der jeweils in einem Sektor und einer Region durchgeführten, simulierten Änderungen der Handelspolitik approximiert.

Auf Basis dieser Meta-Daten wurde ein lineares Regressionsmodell geschätzt, welches den vielfältigen Interaktionseffekten wichtiger Modellspezifikationen innerhalb angewandter Gleichgewichtsmodelle, die gegenwärtig zur globalen Politikfolgenabschätzung eingesetzt werden, Rechnung trägt. Ergebnisse dieser Meta-Analyse haben typische Politikszenarien und zentrale Modelleigenschaften identifiziert, welche einen Großteil der Varianz innerhalb der modellbasierten Literatur zu Politikfolgen der WTO-Doha-Runde DohaRunde systematisch erklären. Der durchschnittliche simulierte Wohlfahrtsgewinn für eine vollständige Abschaffung aller Zölle, Exportsubventionen und preisgebundenen Stützungsmaßnahmen weltweit wird daher je nach verwendeter Modellspezifikation zu einem weltweiten Gewinn zwischen $150 \mathrm{Mrd}$. US-Dollar und 280 Mrd. US-Dollar bei einem Mittelwert von 200 Mrd. USDollar führen; die durchschnittlich simulierte regionale Verteilung dieser Gewinne kann dem umfangreichen Tabellenwerk der Arbeit entnommen werden. Diese simulierten Werte hängen aber unmittelbar mit bestimmten Modellannahmen zusammen und dürfen daher nur in sofern als Messungen interpretiert werden, wie diese Annahmen als realistisches Abbild der Wirklichkeit gelten können - was jedoch auf Basis der theoretischen Diskussion innerhalb der ökonomischen Literatur beispielsweise für die zentrale ArmingtonAnnahme keineswegs gegeben ist.

Die Resultate der Forschungsarbeit können innerhalb der politischen Diskussion helfen, einerseits überhöhte Erwartungen an die Aussagekraft von Simulationsmodellen zu dämpfen und andererseits fundamentale Skepsis zu be- 
seitigen, wodurch die anhand simulierter Ergebnisse gewonnenen Erkenntnisse für politische Verhandlungen effizienter genutzt werden können. Dies weist den eingeschlagenen methodischen Weg als viel versprechende Pionierarbeit aus; die Anwendung der Meta-Analyse zur quantitativen Evaluation von Gleichgewichtsmodellen stößt sowohl bei den Modellentwicklern als auch bei den Modellanwendern auf großes Interesse.

Mit verfügbaren partiellen und allgemeinen Gleichgewichtsmodellen wurden ferner eigene Simulationen erzeugt, um Input-Output-Daten ohne fehlende Werte und ohne Mess- oder Klassifikationsfehler zu generieren. Zukünftige Forschung soll klären, wie die Methode der Meta-Analyse mit Response-Surface-Methoden verbunden werden kann, was einerseits die Erzeugung von detaillierten Response-Surfaces für wichtige Parameter (z.B. Armington-Elastizitäten) ermöglicht und es andererseits erlauben wird, die vorhandenen Lücken aufgrund fehlender Informationen innerhalb des Meta-Datensatzes durch Imputation zu schließen. 
Sebastian Hess - 978-3-631-75694-2

Downloaded from PubFactory at 01/11/2019 02:52:17AM

via free access 


\section{Einleitung und Fragestellung}

Am 27. Juli 2007 wurde das Inselkönigreich Tonga, eine der kleinsten und geographisch abgelegensten Volkswirtschaften der Erde, als 151. Mitglied in die Welthandelsorganisation (WTO) aufgenommen. Hieran wird deutlich, dass sich inzwischen die meisten Nationen dazu bekennen, einen freieren und faireren Welthandel verwirklichen zu wollen. Eine Umsetzung der ökonomischen Forderung nach Freihandel sollte damit grundsätzlich die besten politischen Voraussetzungen haben, welche sich in der Geschichte bisher jemals boten.

Die WTO-Doha-Runde ist gemessen an der Zahl der teilnehmenden Staaten der bislang ambitionierteste Versuch zur Reduktion globaler Handelshemmnisse uberhaupt, wobei die meisten tarifären Handelshemmnisse im Bereich des Weltagrarhandels zu finden sind. Aber diese Doha-Runde ist während ihres Verlaufs auch zu einem Synonym für das sowohl gepriesene als auch gefürchtete allgemeine Phänomen der Globalisierung geworden (Lamy 2007).

Die Ereignisse im Umfeld des G-8 Gipfels in Heiligendamm im Juni 2007, wo zuletzt Rettungsversuche für die Doha-Runde ergebnislos verliefen, zeigen eindringlich, dass Globalisierung und globale Handelsliberalisierung auch dann nicht voneinander zu trennen sind, wenn der Stand der WTO-Verhandlungen unübersehbare Anzeichen schwerer Rückschläge erkennen lässt (Lamy 2007): Ein politisches Scheitern dieser WTO-Runde würde grundsätzlich kaum etwas daran ändern, dass Fragen der globalen Handelsliberalisierung aufgrund der zunehmenden Verflechtung der Weltwirtschaft vermutlich eines der beherrschenden wirtschaftspolitischen Themen des 21. Jahrhunderts bilden werden (Copenhagen Consensus 2007).

Tom Hertel formuliert Konsequenzen dieser Entwicklung aus wissenschaftlicher Sicht in der Einleitung zu dem Benutzerhandbuch des GTAP-Modells folgendermaßen: „As the world economy becomes more integrated, there is an increasing demand for quantitative analyses of policy issues on a global basis“ (Hertel 1997). Ökonomen, sofern sie quantitative Analysen zu Fragen des Welt(agrar)handels veröffentlichen, sind demnach nicht nur Beobachter sondern unmittelbar Teil der politischen und ökonomischen Globalisierungsprozesse.

Könnte dabei der Verlauf der Doha-Runde etwa unter einem Mangel an quantitativen Analysen über die Auswirkungen globaler Handelsliberalisierung gelitten haben? Auf den ersten Blick sicher nicht. Den politischen Protagonisten in Doha, Cancun, Hongkong und auch in Heiligendamm hat sicher kein Verständnis dafür gefehlt, dass für die Welt insgesamt volkswirtschaftich viel auf dem Spiel steht (Lamy 2007). 
Insbesondere zu Beginn der Doha-Runde im Jahr 2001 machten weit über die wissenschaftliche und wissenschaftsnahe ökonomische Literatur hinaus empirische Ergebnisse über die $\mathrm{zu}$ erwartenden Auswirkungen weltweiter Handelsliberalisierung von sich reden. Insbesondere Menschen in den Entwicklungsländern sowie in den am wenigsten entwickelten Ländern sollten, so das große Versprechen der Doha-Development-Agenda, von den Ergebnissen dieser WTO-Runde profitieren: "Abolishing all trade barriers could boost global income by $\$$ US 2.8 trillion and lift 320 million people out of poverty by 2015" (Moore 2002).

In der Tat haben viele internationale Organisationen, nationale Forschungseinrichtungen und Universitäten empirische Analysen mit dem Ziel erstellt, die Einkommens- und Wohlfahrtseffekte unterschiedlicher Politikoptionen während dieser Welthandelsrunde zu errechnen. Das Mittel der Wahl sind dabei stets angewandte Gleichgewichtsmodelle. Sie stellen den Versuch seitens der Wissenschaft dar, Modellvorstellungen der Handelstheorie in Form von Gleichungssystemen $\mathrm{zu}$ formulieren und anhand realer Daten und unter Einbeziehung hypothetischer Politikänderungen $\mathrm{zu}$ lösen. Die grundsätzliche Funktionsweise angewandter Gleichgewichtsmodelle wird in Kapitel 2 eingehend erläutert.

Auch Nicht-Ökonomen dürften dabei während der ersten Jahre der DohaRunde aus der Tagespresse den Eindruck gewonnen haben, dass die globalen Liberalisierungsgewinne lediglich darauf warten, von den verhandlungsfuhrenden Politikern für die Menschheit als reiche Ernte eingefahren zu werden. Im Angesicht geradezu astronomischer Ergebnisse vieler ökonomischer Studien wurde dabei zunächst die Frage nach der regionalen Verteilung dieser Gewinne vernachlässigt: „Significant gains would be derived from implementation of the [Harbinson]draft proposal - $100,000,000,000 \$$ in global welfare gains. ... However, ... some African countries may even experience some losses because of terms of trade effects. Nevertheless, the ample gains, particularly in the developed countries, should suffice to ensure a win-win situation for everybody" (Gersfelt, Lind, Frandsen, Jensen und Yu 2003).

In dieser Atmosphäre politischer und numerischer Euphorie, als errechnete Gewinne zum Greifen nahe schienen und Lösungen für einige der gewaltigsten und dringendsten globalen Probleme versprachen, überraschte es jedoch auch nicht, dass zumindest einige Politiker die Simulationsergebnisse angewandter Gleichgewichtsmodelle verwendeten, um gleichsam im Windschatten globaler Liberalisierungsgewinne höchst nationale Interessen $\mathrm{zu}$ verfolgen. Und es überrascht ebenso wenig, dass den politischen Gegnern solch politischer Interessen auch die Simulationsergebnisse zu einem Dorn im Auge wurden: „Drusilla Brown, Alan Deardorff, and Robert Stern ... estimate that complete elimination of all trade barriers in the world would add $\$ 1.9$ trillion (about 5\%) to the 
world's gross economic product by $2005 .$. leaving the casual reader with the sense that free trade benefits are pretty much in the bank - ... if only we would adopt the correct trade policies. But a closer look reveals that ... BrownDeardorff-Stern never rebut a single criticism of global liberalization; instead, they simply assume that each criticism is false or irrelevant to begin with. ... In spite of this, supporters of the Bush Administration's fasttrack proposal ... cite the Brown-Deardorff-Stern estimate to justify the rapid trade liberalization that fast track would promote“" (Dorman 2001).

Aufgrund der offensichtlich hohen politischen Brisanz empirischer Politikfolgenabschätzungen auf Basis angewandter Gleichgewichtsmodelle wurde die politische Kritik an bestimmten politischen Vorschlägen zur multilateralen Handelsliberalisierung während des Verlaufs der Doha-Runde auch unmittelbar mit Kritik an angewandten Gleichgewichtsmodellen als der ökonomischen State-of-the-art-Methode vermischt: „By pretending to see into the future and putting numbers on the 'welfare gains', ... findings of models, presented as fact, put ... those advocating a more cautious approach to liberalisation on the defensive ... there is no reason to take seriously the exact numbers about gains from liberalisation that they churn out, ... their order of magnitude could be estimated without resorting to the computer in any case" (Taylor und von Arnim 2006).

Aber nicht nur für politische Entscheidungsträger dürften die zu erwartenden Wohlfahrtseffekte multinationaler Handelsliberalisierung von großem Interesse sein, denn Handelsliberalisierung betrifft nicht in erster Linie abstrakte Größenordnungen der Volksvermögensrechnung, sondern vor allem zunächst die Arbeitsplätze und Einkommen vieler einzelner Menschen in allen Erdteilen. Es erscheint daher legitim, von den Protagonisten der Welthandelsrunden eine ausgewogene und verantwortungsvolle Vertretung der Interessen von Produzenten und Konsumenten, sprich Menschen, in ihren Ländern zu verlangen. Es erscheint aber ebenso legitim, von Ökonomen zu erwarten, sofern sie empirische Analysen zur Auswirkung multinationaler Handelsliberalisierung veröffentlichen, dass sie bei Erstellung dieser Analysen die höchsten wissenschaftlichen Standards anlegen, welche auf Basis der existierenden Erkenntnisse und technischen Möglichkeiten zu einem bestimmten Zeitpunkt umsetzbar sind: „BrownDeardorff-Stern have indeed done nothing more than drop a rabbit into a hat and then pull it out. Old tricks cannot serve as a serious basis for policy analysis. Moreover, the stakes are too high for theatrics: the well-being of billions of workers and entire economies are at stake. All citizens deserve a critical analysis of the costs and benefits of globalization, an analysis that holds up to honest scrutiny" (Dorman 2001).

Im Angesicht der sich als extrem schwierig und kontrovers gestaltenden WTO Verhandlungen muss konstatiert werden, dass es Ökonomen weltweit 
während des Verlaufs der Doha-Runde nicht gelungen ist, angewandte Gleichgewichtsmodelle als eine wenn nicht fehlerfreie, so doch weitgehend objektive Methode zur quantitativen Politikfolgenabschätzung zu etablieren, deren allgemeine Aussagekraft von handelspolitischen Einzelinteressen nicht jeder Zeit grundsätzlich in Frage gestellt werden kann.

Einige der bedeutendsten Denker der Ökonomie würden dabei moderne angewandte Gleichgewichtsmodelle möglicherweise sogar für das tollkühne Resultat eines grundsätzlichen Fehlverständnisses ökonomischer Theorie halten: „Es scheint [dem Physiker und Chemiker] meist unverständlich, dass etwa der Nationalökonom seine Zeit darauf verwendet, den physikalischen äußerlich ähnliche Theorien zum Beispiel in der Form von Gleichungssystemen auszuarbeiten, obwohl dieser zugibt, dass er die Daten, die er in diese Gleichungen einsetzen müsste, um sie zu lösen, nie feststellen kann" (von Hayek 1963, S. 15).

Dabei konnte von Hayek die gewaltigen Fortschritte im Bereich der Datenerhebung und Informationsverarbeitung nicht kennen, welche mit dem Siegeszug von Computern und Datennetzwerken möglich wurden. Jedoch bleibt andererseits fraglich, ob ihn dieses angesichts der tatsächlichen Komplexität realer Wirtschaftsbeziehungen zu einer Revision dessen, was Ökonomie seiner Auffassung nach nicht sein sollte, bewogen hätte, oder ob er den technischen Aufwand zur Erstellung von Simulationsmodellen vielmehr als Bestätigung seiner Argumentation gewertet hätte.

Ähnlich formulierte Keynes seine berühmte Definition von Ökonomie: „The theory of economics does not furnish a body of settled conclusions immediately applicable to policy. It is a method rather than a doctrine, an apparatus of the mind, a technique of thinking which helps its processors to draw correct conclusions" (Keynes 1922).

Diese Aussagen lassen erahnen, dass ökonomische Theorie ursprünglich nicht entwickelt wurde, um sie in Form von Simulationsmodellen zusammenzufügen und zur Errechnung globaler Wohlfahrtseffekte zu nutzen. Moderne angewandte Gleichgewichtsmodelle sind dennoch zweifelsohne auch solche apparati of the mind und sie sind anders als die meisten menschlichen Gehirne in der Lage, diesen Apparat unter Einbeziehung von Millionen einzelner Beobachtungen zu betreiben. Auch Keynes und von Hayek würden sicher zugestehen, dass diese technischen Möglichkeiten grundsätzlich gewaltige Chancen zur Lösung von globalen wirtschaftspolitischen Fragen bieten, deren Komplexität selbst die analytischen Fähigkeiten von Nobelpreisträgern übersteigt.

Aber genau hier ist Vorsicht geboten: Kein Apparat kann (bisher!) eigenständig korrekte Schlüsse ziehen. Ein Apparat kann aber eventuell denjenigen, die ihn betreiben, dabei helfen, über komplexe wirtschaftspolitische Probleme 
eben diese korrekten Schlüsse zu entwickeln - vorausgesetzt, die Bedienung dieses Apparats wird beherrscht!

Experten im Bereich der Entwicklung angewandter Gleichgewichtsmodelle sind sich dieser Problematik sehr wohl bewusst: „When economists model trade policy for the policy community... they face two critical challenges. The first involves developing a reasonable, though stylized representation of complex ... relationships. ... The second challenge involves presentation and explanation of the results" (Francois 2004, S. 75f.).

Es verwundert daher nur wenig, dass die mit steigender Rechnerkapazität zunehmende Komplexität der verwendeten „Apparate“ im Bereich der simulationsmodell-basierten ökonomischen Literatur seit jeher den Vorwurf chronischer Intransparenz provoziert hat und inzwischen für zeitgenössische Simulationsexperimente unter der Doha-Runde selbst deskriptive Vergleiche einander ähnlicher Studien nur sehr eingeschränkt durchführbar sind (Sumner und Tangermann 2002).

Die vorliegende Meta-Analyse möchte genau an dieser Stelle zur modellgestützten quantitativen Politikanalyse beitragen. Dies soll jedoch nicht geschehen, indem den bestehenden Modellen ein weiteres hinzugefugt wird. Es soll ebenso wenig versucht werden, an den normativen Aussagen vieler Studien inhaltlich zu zweifeln oder bestehende Modellkonzepte qualitativ gegeneinander abzugrenzen, geschweige denn das theoretische Gebäude der ökonomischen Handelstheorie anzutasten. Das Ziel der vorliegenden Analyse besteht vielmehr darin, die bereits vorhandenen empirischen Ergebnisse existierender angewandter Gleichgewichtsmodelle während der Doha-Runde als den Stand der Forschung auf diesem Gebiet $\mathrm{zu}$ betrachten und sie einer eingehenden empirischen Evaluation zu unterziehen. Dadurch sollen Antworten auf die folgenden, inhaltlich eng miteinander verwobenen Forschungsfragen zu modellgestützter Politikfolgenabschätzung im Spannungsfeld zwischen Wissenschaft und politischem Diskurs gefunden werden:

- Transparenz: Kann das Zustandekommen von Simulationsergebnissen angewandter Gleichgewichtsmodelle zu Fragen der Wohlfahrtswirkungen multiregionaler Handelsliberalisierung systematisch erklärt und über Studien und Modelle hinweg miteinander verglichen werden?

- Empirie: Welche Aussagen können anhand bisher existierender Studien über durchschnittlich zu erwartende, simulierte Einkommens- (bzw. Wohlfahrts-)veränderungen in

a) der Welt als geschlossener Volkswirtschaft und

b) innerhalb einzelner Länder als Folge von Handelsliberalisierung getroffen werden? 
- Interpretation: Unter welchen Bedingungen werden diese Veränderungen in der Wirklichkeit eintreten?

- Methodische Weiterentwicklung: Durch welche Voraussetzungen könnte die allgemeine Akzeptanz angewandter Gleichgewichtsmodelle und ihrer Ergebnisse als wissenschaftliche Methode zur quantitativen Politikfolgenabschätzung verbessert werden?

Zur Beantwortung dieser Fragen ist es zunächst notwendig, einen Überblick über den Aufbau und die Funktionsweise angewandter Gleichgewichtsmodelle zu erlangen und den Stand der Forschung hinsichtlich bekannter methodischer Stärken und Schwierigkeiten im Zusammenhang mit der Erstellung simulierter Politikfolgenabschätzungen auf Basis dieser Modelle zu erarbeiten (Kapitel 2).

Die Methode der Meta-Analyse', gemäß Stanley (2001) die beste und gegenwärtig wohl einzige empirische Möglichkeit im Bereich der ökonomischen Literatur, um „die Spreu vom Weizen zu trennen“, sprich, quantitative Ergebnisse über Studien hinweg zu vergleichen und für Unterschiede zwischen den Ergebnissen multivariat zu kontrollieren, soll wie in Kapitel 3 erläutert auf angewandte Gleichgewichtsmodelle und ihre simulierten Ergebnisse während der Doha-Runde übertragen werden, um Antworten auf die genannten Forschungsfragen zu finden.

Kapitel 4 präsentiert und diskutiert Ergebnisse dieser Meta-Analyse. Kapitel 5 stellt die Ergebnisse der Meta-Analyse in den Kontext existierender und neuer Erfahrungen mit Sensitivitätsanalyse und Response-Surface-Design und illustriert anhand einfacher Gleichgewichtsmodelle, wie ökonometrische MetaModellierung genutzt werden kann, um auf Basis einer Meta-Analyse bestehende Modellansätze zusammenzuführen, robuste empirische Aussagen über Modellansätze hinweg zu gewinnen und simulierte Ergebnisse leichter interpretierbar zu machen. Kapitel 6 blickt auf zukünftige Forschungsfragen, Kapitel 7 schließt die Analyse zusammenfassend ab.

1 Die etymologische Bedeutung des griechischen Wortes „meta“ umfasst so viel wie „hinter, zwischen, oberhalb“ und bildet u.a. die Wurzel für das deutsche Wort „mit" (gemäß Etymologie- Duden). 


\section{Evaluation angewandter Gleichgewichtsmodelle: Stand der Forschung}

Im vorangegangenen Kapitel wurde die große Bedeutung angewandter Gleichgewichtsmodelle und der durch sie simulierten Ergebnisse für politische Diskussionen und evtl. für politische Entscheidungen deutlich. Gleichzeitig scheint die Antizipation dieser Ergebnisse innerhalb des politischen Diskurses von unterschiedlich hoher durchschnittlicher Akzeptanz begleitet zu sein. Dieses Kapitel fasst die bestehenden wissenschaftlichen Erkenntnisse hinsichtlich folgender Fragen zusammen: 1.) Wodurch sind zur Politikfolgenabschätzung eingesetzte angewandte Gleichgewichtsmodelle gekennzeichnet? 2.) Was können sie aufgrund ihres allgemeinen Aufbaus gegenwärtig leisten und 3.) worin liegen aus wissenschaftlicher Sicht gegenwärtig ihre größten methodischen Herausforderungen?

\subsection{Untersuchungsgegenstand: Was sind angewandte Gleich- gewichtsmodelle?}

Angewandte allgemeine und partielle Gleichgewichtsmodelle stellen gegenwärtig den umfassendsten Versuch seitens der Wissenschaft dar, die Weltwirtschaft modellhaft abzubilden und auf Basis dieser Modelle quantitative Prognosen zu erstellen. Typische Elemente dieser Modelle sind erstens ein Gleichungssystem, welches typische funktionale Zusammenhänge der Handelstheorie abbildet; zweitens eine Datenbasis, welche relevante Daten über Produktions- und Handelsmengen, Finanzströme, Faktorausstattung, Zölle, Quoten, etc. enthält und meist ein Basisjahr oder einen Basiszeitraum abbildet; drittens stellen eine mehr oder weniger große Zahl exogener Parameter eine wichtige Komponente angewandter Gleichgewichtsmodelle dar. Für angewandte allgemeine Gleichgewichtsmodelle (nachfolgend synonym zu Computable General Equilibrium, CGE) sind dies meist Substitutions- und Transformationselastizitäten; für partielle Gleichgewichtsmodelle (nachfolgend synonym zu Partial Equilibrium, PE) sind diese exogenen Parameter insbesondere Angebots- und Nachfrageelastizitäten. Ausfuhrliche formale Darstellungen dieses Grundprinzips angewandter Gleichgewichtsmodell finden sich bei u.a. bei Shoven und Whalley (1984), Robinson (1989), Ginsburgh und Keyzer (1997) sowie Francois und Reinert (1997). 
Die zum Einsatz kommenden Modelle bezeichnet man aufgrund ihrer Verbindung aus theoretischer Struktur und empirisch fundierter Datengrundlage auch als „angewandte“ Modelle. Van Tongeren, van Meijl und Surry (2001) definieren typische Charakteristika angewandter Gleichgewichtsmodelle, wie sie in die vorliegende Meta-Analyse eingehen sollen: multiregional im Fokus (d.h. mindestens ein Land/Region handelt mit mindestens einer weiteren Region), relevant für den Agrarsektor, den Agrarhandel oder auf natürlichen Ressourcen beruhenden Aktivitäten, Gleichgewichtscharakter, partiell oder allgemein; angewandte Ausrichtung, starke Orientierung an (Handels-)Politikevaluation (van Tongeren, van Meijl und Surry 2001).

Da aufgrund der weltweit besonders stark protektionierten Agrarmärkte in den meisten Simulationsstudien zur Doha-Runde ein starker Bezug zum Agrarsektor vorliegt, werden angewandte Gleichgewichtsmodelle mitunter auch als „Agrarsektormodelle“ bezeichnet (Heckelei, Witzke und Henrichsmeyer 2000). Partielle Gleichgewichtsmodelle, welche zur Analyse von Handelspolitik verwendet werden und ausschließlich auf den Agrarsektor fokussieren, werden hiernach folglich ebenfalls als ,angewandte Gleichgewichtsmodelle“ bezeichnet und in die Analyse eingeschlossen.

Die Bezeichnung „Agrarsektormodelle“ ist jedoch weiter gefasst: Auf Betriebs- und Regionalebene finden unter dieser Bezeichnung überwiegend Ansätze aus dem Bereich der linearen Optimierung sowie der Multi-Agentenmodelle Anwendung. Keiner dieser beiden Ansätze ist zur Modellierung von Handelspolitik verbreitet. Nationale und internationale Politikänderungen mit dem Ziel einer wirklichkeitsgetreuen Folgenabschätzung werden hingegen fast ausschließlich durch partielle oder allgemeine Gleichgewichtsmodelle abgebildet (Anderson und van Wincoop 2001) - mit einer wichtigen und ebenfalls innerhalb der Literatur zahlreich vertretenen Ausnahme: den GravityModellen. Gravity-Modelle werden ökonometrisch anhand realer Daten geschätzt, bilden Handelsströme zwischen Ländern ab und ermöglichen Aussagen über Veränderungen dieser Handelsströme. Sie weisen ebenfalls angewandten Charakter auf, aber Gravity-Modelle sind fur ex ante Simulationen geplanter Politikänderungen und insbesondere für die Errechnung von Wohlfahrtseffekten ungeeignet, da sie kaum Interaktionen von Handelsströmen mit anderen volkswirtschaftlichen Schlüsselvariablen abbilden können (Anderson und van Wincoop 2001). Gravity-Modelle sind mit der ökonomischen Handelstheorie darüber hinaus nicht unmittelbar verwandt und in ihrem Aufbau zu Gleichgewichtsmodellen grundverschieden, weshalb sie von der hier durchgeführten Meta-Analyse ausgenommen sind; eine Meta-Analyse von Gravity-modellbasierten Studien wird gegenwärtig von Cipollina und Salvatici (2006) durchgeführt. 
Hinsichtlich der durch van Tongeren, van Meijl und Surry (2001) eingegrenzten, angewandten Gleichgewichtsmodelle müssen verschiedene Betrachtungsebenen begrifflich unterschieden werden, wofür sich jedoch innerhalb der Literatur keine einheitliche Nomenklatur herausgebildet hat. Die nachfolgenden Definitionen sind am Bedarf der vorliegenden Meta-Analyse ausgerichtet und daher evtl. vom Gebrauch in anderen Bereichen der Literatur zu unterscheiden.

Modelle und Modellkonzepte: Grundsätzlich bildet jedes Simulationsexperiment mit seiner spezifischen Versuchsanordnung von Gleichungen, Daten und spezifizierten Politikänderungen ein eigenes Modell, da durch den jeweiligen experimentellen Aufbau ein ganz bestimmter Aspekt der Wirklichkeit (z.B. Entkopplung der Direktzahlungen der CAP) abgebildet werden soll. Innerhalb der Literatur zu angewandten Gleichgewichtsmodellen wird jedoch unter einem „Modell“" meist das auf eine bestimmte Forschergruppe zurückgehende und unter Akronymen bekannte, allgemeine Konzept aus Basisdaten, Parametern und Gleichungen verstanden (z.B. „das GTAP-Modell“, „ESIM“", „MIRAGE“, „WATSIM“", „Aglink“, „ATPSM“, „Linkage“, „FAPRI“, etc.). Diese Modellkonzepte werden meist ständig weiterentwickelt (z.B. GTAP, FAPRI) und an neue Fragestellungen angepasst. Mitunter lässt man bestimmte Modellkonzepte aber auch in anderen aufgehen. Ferner haben einige Modellkonzepte nach ihrer Entwicklung nie wieder eine Anwendung erfahren, was typischerweise solchen Modellkonzepten droht, die als Insellösungen entstanden sind (Doktorarbeiten) und nicht durch ressourcenstarke Forscherteams und politiknahe Netzwerke neuen Anwendungen zugeführt werden (Bureau 2006).

Im Falle des sehr weit verbreiteten Standard-GTAP-Modells (Hertel 1997) haben sich im Zeitverlauf viele spezialisierte Versionen gebildet, welche teilweise unter eigenen Akronymen bekannt sind (GTAP-Agr, GTAP-E, GTAPdyn) oder andererseits sehr tiefgreifende Modifikationen des Standardmodells vornehmen, ohne dies durch ein eigenes Akronym zu kennzeichnen (z.B. das Modell von Francois, van Meijl und van Tongeren 2005 mit steigenden Skalenerträgen und unvollkommenem Wettbewerb in Industrie- und Dienstleistungssektoren sowie Akkumulation des Kapitalstocks). Darüber hinaus können sehr unterschiedliche Simulationsexperimente durchgeführt werden, indem das jeweilige Politikszenario beispielsweise unter Veränderung der Modell-Closure (vgl. Hertel 1997) durchgeführt wird.

Aus diesen Gründen wird nachfolgend der Begriff "Modellkonzept“ verwendet, sofern geläufige Modellakronyme allgemein bezeichnet werden sollen und grundsätzlich alle anhand dieser Modellkonzepte durchführbaren Simulationsexperimente eingeschlossen sind; der Begriff „Modell“" wird hingegen nur dann verwendet, wenn eine Aussage auch allgemein für ökonomische bzw. wissenschaftliche Modelle gelten kann, wobei dann Modellkonzepte und Simulationsexperimente mit eingeschlossen sind. 
Modellansätze: Bezieht sich die Betrachtungsebene nicht auf Modellkonzepte, sondern auf wichtige Grundannahmen innerhalb von Modellkonzepten, so wird nachfolgend der Begriff des „Modellansatzes“" verwendet: Die Modellkonzepte ATPSM, ESIM und AGLINK folgen beispielsweise alle dem Modellansatz des partiellen Gleichgewichtsmodells. GTAP-dyn und MIRAGE folgen dem dynamischen Modellansatz. Die Kriterien hinsichtlich dessen, was einen Modellansatz bildet, sind dabei nicht klar festgelegt, sondern werden durch Annahmen gebildet, welche innerhalb der Literatur als besonders wichtig beschrieben werden oder innerhalb der ökonomischen Theorie zentrale Modellvorstellungen charakterisieren (beispielsweise IRTS versus CRTS). Die Auswahl dieser Annahmen vor Durchführung eines Simulationsexperiments (sog. ,model pre-selection“, Hertel 1999) determiniert möglicherweise bereits die späteren Simulationsergebnisse entscheidend, wobei jedoch bisher kaum über Modellkonzepte hinweg verallgemeinerbare empirische Erkenntnisse vorliegen (Hertel 1999). Die Überprüfung der Hypothese, dass ein bestimmter Modellansatz auch über sehr verschiedene Modellkonzepte hinweg einen statistisch signifikanten Einfluss auf die Simulationsergebnisse hat, ist ein Untersuchungsgegenstand dieser Meta-Analyse.

Wichtige Modellansätze sind dynamische versus komparativ-statische Modellierung, die Annahme vollkommenen versus unvollkommenen Wettbewerbs (Robinson 1989, Hertel 1999) sowie die Annahme perfekter Substitution gehandelter Güter versus der sog. Armington-Annahme (Armington 1969). Diese Armington-Annahme kommt in den meisten angewandten Gleichgewichtsmodellen vor, spielt aber innerhalb der übrigen ökonomischen Literatur nur eine untergeordnete Rolle (Xu 2002), weshalb sie nachfolgend kurz erläutert werden soll:

Ausgangspunkt theoretischer Betrachtungen zum Welthandel ist meist das Modell von Ricardo (1821), welches später insbesondere durch Heckscher und Ohlin (siehe z.B. Ohlin 1933) formalisiert wurde. Gemäß diesem Modell führt Handel zu einer Spezialisierung der handeltreibenden Länder entlang ihrer unterschiedlichen relativen Faktorausstattung. Dies bedeutet, dass ein relativ kapitalreiches Land seine Ressourcen besser nutzen kann, indem es ein relativ kapitalintensives Gut exportiert und ein relativ arbeitsintensives weiteres Gut aus einem Land mit relativ reichhaltiger Ausstattung an Arbeitskrätten importiert (Ricardo 1821).

In diesem Modell stehen politische Handelshemmnisse (Zölle) einer weitergeführten Spezialisierung beider (bzw. aller beteiligten) Länder im Wege und ein Abbau dieser Zölle fuhrt zu steigender Spezialisierung und damit zu einer Realisierung von Spezialisierungs- und Handelsgewinnen in den beteiligten Ländern. 
Dieses Modell, obwohl Grundlage fur die weitere Entwicklung der Handelstheorie, ist nur sehr bedingt in der Lage, tatsächlich beobachtete Handelsströme empirisch zu erklären (Feenstra 2003). Ein wichtiger Grund hierfür ist die Tatsache, dass einige Grundannahmen dieses Modells sehr restriktiv sind: Entgegen den Annahmen dieses Modells sind die Produktionstechnologien nicht in allen Ländern gleich, Ressourcen sind nicht vollkommen immobil und Konsumenten haben möglicherweise nicht in allen Ländern die gleichen Präferenzen (Corden 1974). Hinzu kommt, dass man innerhalb internationaler Handelsstatistiken zumindest bei aggregierten Daten (Sarker und Surry 2006) beobachtet, dass offenbar gleiche Produkte zwischen miteinander handelnden Ländern sowohl exportiert als auch importiert werden (Intra-Industry-Handel, IIT).

Die Armington-Annahme unterstellt, dass sich Produkte des gleichen Sektors bzw. der gleichen Industrie anhand ihres Ursprungslandes unterscheiden und deshalb in den Augen von Konsumenten einander nur unvollkommen substituieren sowie gegenüber inländisch produzierten Gütern des gleichen Sektors ebenfalls nur unvollkommene Substitute darstellen.

Die sog. Armington-Annahme wird in diesem Zusammenhang seit jeher kritisch diskutiert (Brown 1987, Alston und Hurd 1990, Hertel 1997, Sarker und Surry 2006).

Die Armington-Annahme ist nicht die einzige Möglichkeit zur Abbildung von IIT innerhalb angewandter Modelle, aber sie stellt insbesondere für multiregionale, multisektorale Modelle (z.B. GTAP) eine besonders praktikable Lösung dar, da es nicht notwendig ist, Daten über den Handel mit Produkten auf Firmenebene zu erheben. Vielmehr wird unterstellt, dass sich „deutsche“ Autos von ,japanischen" hinreichend unterscheiden, während die Unterschiede zwischen einzelnen Automarken ignoriert werden, um nicht Export- und Importdaten von Volkswagen, Toyota, etc. verwenden zu müssen. Außerhalb angewandter Gleichgewichtsmodelle sind, insbesondere im Rahmen theoretischer Analysen, sog. Love of Variety (LOV) Modelle zur Modellierung von IIT verbreitet (Xu 2002). LOV-Modelle müssen sorgfältig von Modellen mit Armington-Annahme getrennt werden: Während die Armington-Annahme die Zahl der Product Varieties je Land fixiert und je Sektor auf ein Produkt begrenzt, wächst bei LOV-Modellen die Zahl der Product Varieties proportional mit der Größe eines Landes. Xu (2002) stellt heraus, dass der wichtigste Unterschied zwischen beiden Modellansätzen in der Höhe der Substitutionselastizität liegt und dass ein Armington-Modell mit unendlicher Substitutionselastizität hinsichtlich simulierter Ergebnisse der Verhaltensweise eines LOV-Modells nahe kommt. Ardelean (2007) weist darauf hin, dass im LOV-Modell keine Terms-of-Trade (ToT) Effekte vorkommen, während diese im ArmingtonModell besondere Bedeutung besitzen. Ardelean (2007) entwickelt ein Handels- 
modell mit einer CES-Präferenzstruktur, welche das LOV-Modell und das Armington-Modell verbindet.

Die Armington-Annahme ist typischer Weise auf der Nachfrageseite eines Modells angesiedelt und wird meist anhand von genesteten CES-Funktionen implementiert (Francois und Hall 1997). Dabei werden während der Budgetallokationsentscheidung der (des repräsentativen) Konsumenten innerhalb eines Simulationsdurchlaufs zunächst importierte Güter aus unterschiedlichen Ländern anhand einer Substitutionselastizität miteinander kombiniert und nachfolgend wird dieses importierte Güterbündel mit dem inländischen Produkt des gleichen Sektors kombiniert, sodass ein inländisch nachgefragtes Güterbündel für ein aggregiertes Armington-Produkt entsteht.

Insgesamt ergibt sich für verarbeitete Produkte durch die Armington-Annahme in angewandten Gleichgewichtsmodellen ein operationalisierbares und im Vergleich zum Heckscher-Ohlin-Modell maßvoll realistisches Abbild tatsächlich beobachteter Handelsströme, während die Armington-Annahme im Falle einiger primärer Agrarprodukte als Hypothese empirisch abgelehnt wurde (Alston und Hurd 1990). Viele Autoren haben nachfolgend ArmingtonSubstitutionselastizitäten empirisch geschätzt oder anhand realer Daten kalibriert (z.B. Gehlhar 1997).

Eine unendliche Substitutionselastizität bedeutet dabei, dass die importierten Produkte eines Sektors das entsprechende heimische Produkt perfekt substituieren. Dies bedeutet auch, dass eine größere Zahl an Produkten aus mehreren Ländern die Bedeutung jedes einzelnen Produktes mit regionaler Herkunft erodiert. Durch diesen Zusammenhang wird bereits intuitiv klar, dass die Armington-Annahme eine entscheidende Abweichung von dem durch Ricardo begründeten Ansatz der Spezialisierung entlang relativer Produktionsvorteile darstellt: Die Armington-Annahme verleiht jedem Land in jedem seiner Sektoren Marktmacht für seine Im- und Exporte. Dadurch wird die Annahme eines „kleinen Landes“ (= keine ToT-Effekte) innerhalb eines Modells faktisch unmöglich und für jedes Land müssen diese ToT-Effekte theoretisch und empirisch berücksichtigt werden (Brown 1987).

Als „Modellansätze“ werden somit insgesamt solche Modellannahmen bezeichnet, deren starker Einfluss auf den Ausgang eines Simulationsexperiments bereits bekannt ist oder aufgrund theoretischer Zusammenhänge erwartet werden kann.

Simulationsexperimente (nachfolgend synonym verwendet zu „Szenarien“, „Simulationen“, „Versuchsaufbau“): Zum Zweck der Politikanalyse werden modellexogene Variablen eines bestehenden Modellkonzepts vor Durchführung einer Simulation modifiziert, um sie an eine bestimmte politische Fragestellung anzupassen. Im Falle einer Zollreduktion heißt dies beispielsweise, dass die Variable "Zollsatz" in einer komparativ-statischen Simulation von ihrem 
Ausgangswert (zum Zeitpunkt $t_{l}$; gelegentlich auch als Baseline bezeichnet) auf einen politisch vorgegebenen Zielwert gesetzt wird, bevor das Gleichungssystem erneut gelöst wird $\left(t_{2}\right)$. Die Differenz der Werte endogener Modellvariablen zwischen $t_{1}$ und $t_{2}$ wird dann als Simulationsergebnis interpretiert.

Über Modifikationen modellexogener Variablen (z.B. Zölle) hinaus können jedoch noch wesentlich umfangreichere Modifikationen an einem Modellkonzept vorgenommen werden, wie beispielsweise Modifikationen des Gleichungssystems. Sofern diese Modifikationen die Spezifikation exogener und endogener Variablen berühren, spricht man von Veränderungen der ModellClosure; berühren diese Veränderungen der Closure wichtige makroökonomische Funktionsweisen des Modells, spricht man von einer Macro-Closure (vgl. Rattsø 1982, Dewatripont und Michel 1987). Das GTAP-Modellkonzept (Hertel 1997) erlaubt beispielsweise die Verwendung einer allgemeinen Closure (für den Modellansatz eines allgemeinen Gleichgewichtsmodells) sowie eine partielle Closure (Modellansatz partielles Gleichgewicht). Die individuelle Anordnung dieser Modellansätze in Kombination mit den verwendeten Daten und Parametern sowie den zu untersuchenden Politikänderungen bilden das durchzuführende Simulationsexperiment.

Der folgende Abschnitt fasst zusammen, zu welcher Art von Politikanalyse sich angewandte Gleichgewichtsmodelle besonders eignen und warum sie wahrscheinlich so große Bedeutung zur ex ante Politikfolgenabschätzung erlangt haben.

\subsection{Stärken angewandter Gleichgewichtsmodelle}

Angewandte Gleichgewichtsmodelle stellen gegenwärtig die einzige ökonomische Methode dar, anhand welcher man im größeren Umfang quantitative Analysen hypothetischer, handelspolitischer Ereignisse durchfüren kann. Keine andere Methode ermöglicht einen theoretisch konsistenten Blick auf so vielfältige wirtschaftliche Zusammenhänge gleichzeitig. Dabei liegt die besondere Stärke solch hypothetischer, szenarienhafter Betrachtungen in der Tatsache, dass keine Beschränkung auf ceteris paribus Betrachtungen existiert, sondern die Interaktionen mehrerer hypothetischer Annahmen mit der übrigen modellhaft abgebildeten Volkswirtschaft simuliert werden können. Solche hypothetischen Simulationen sind jedoch in ihrem Anspruch nicht unbedingt gleichzusetzen mit quantitativen Prognosen einzelner politischer Zielvariablen, es sei denn, ein bestimmtes Simulationsexperiment zielt explizit hierauf ab.

In wie weit sich angewandte Gleichgewichtsmodelle nicht nur zur Simulation hypothetischer Szenarien sondern auch zur Erstellung spezifischer 
Prognosen über in der Realität $\mathrm{zu}$ erwartende Veränderungen bestimmter Messgrößen eignen, ist Gegenstand eines vergleichsweise kleinen Forschungsbereichs, welcher ex post Validierungen bestehender Simulationen vornimmt, d.h. rückwirkende Vergleiche simulierter Prognosen mit in der Realität tatsächlich eingetretenen Effekten durchführt. Diese Frage nach der Prognosequalität der besten Modelle „von gestern“ kann allerdings nur sinnvoll sein, wenn die betreffenden Simulationen auch tatsächlich den Anspruch einer quantitativen Prognose und nicht lediglich eines hypothetischen Szenarios erhoben haben, was aber insbesondere im Bereich von Studien durchaus zu erwarten ist, die im unmittelbaren Dialog mit politischen Entscheidungsträgern entstanden sind.

Vergleichsweise wenige Studien haben allerdings diesen Versuch unternommen, ehemals simulierte Ergebnisse mit den in der Realität später tatsächlich eingetretenen Effekten zu vergleichen (Gehlhar 1997, Nölle und Banse 2002, Kehoe 2002). Diese im Vergleich zu Sensitivitätsanalysen spärlich betriebene Form der Modellevaluation birgt aus methodischer Sicht die große Schwierigkeit, innerhalb der Realität einen Referenzzeitraum ausweisen zu müssen. Dies bedeutet, dass zunächst ein Wirtschaftsbereich identifiziert werden muss, in welchem während einer bestimmten Zeit Bedingungen geherrscht haben, welche den Bedingungen innerhalb eines bestimmten, vor Beginn dieser Referenzperiode durchgeführten Simulationsexperiments vergleichbar sind. Gegen diese wirtschaftspolitische Referenzperiode (z.B. die ersten 5 Jahre nach der EUOsterweiterung, die ersten 10 Jahre nach der Gründung der NAFTA) könnten die ehemals erstellten Simulationsergebnisse ein ex post Benchmarking erfahren, aber es ist offenkundig, dass globale Schocks (internationale Krisen, Naturkatastrophen, Terroranschläge, etc.) von keinem gegenwärtig vorstellbaren Simulationsmodell antizipiert werden können und in Folge dessen auftretende Politik- und Markteffekte die Wirkung gezielt umgesetzter Politikmaßnahmen innerhalb verfügbarer Daten häufig überlagern. Die wenigen existierenden Studien zur ex post Validierung kommen vielmehr zu der vorsichtigen Einschätzung, dass eine um solche unvorhersehbaren Schocks bereinigte Baseline, also ein wie auch immer "geglättetes" Abbild real beobachteter Entwicklungen, durchaus in Einklang mit simulierten Szenarien gebracht werden kann (Nölle und Banse 2002, Panagariya und Duttagupta 2001, Kehoe 2002, Feenstra 2006) und zwar insbesondere dann, wenn Schlüsselparameter (Elastizitäten) empirisch validiert wurden (Gehlhar 1997). Jedoch zeigen sich auch gegenteilige Stimmen, die angewandten Gleichgewichtsmodellen generell mangelnde Prognosequalität attestieren und hierfür auch empirische Belege, wie etwa ältere Simulationsergebnisse und die später in Wirklichkeit beobachteten Effekte nach Gründung der NAFTA anführen (Panagariya und Duttagupta 2001, Feenstra 2006). 
Trotz dieser offenbar eingeschränkten Prognosequalität hat sich quantitative Politikfolgenabschätzung mit Hilfe von Simulationsmodellen zu einem bedeutenden ökonomischen Forschungsgebiet für die ex ante Analyse geplanter Politikmaßnahmen entwickelt, was auf die relative Vorzüglichkeit dieser Methode gegenüber weiteren ökonomischen Ansätzen hindeutet (van Tongeren, van Meijl und Surry 2001, Devarajan und Robinson 2002).

\subsection{Kritik an angewandten Gleichgewichtsmodellen}

Angewandte Gleichgewichtsmodelle stellen eine bedeutende Methode zur ex ante Abschätzung von Politikfolgen dar, und haben innerhalb der Ökonomie eine sehr heterogene methodenkritische Literatur provoziert, welche von unterschiedlichen Ansatzpunkten ausgeht, um Schwachstellen an diesen Modellen aufzuzeigen und methodische Weiterentwicklungen vorzustellen. Hinsichtlich der eingangs formulierten Forschungsfragen können diese Ansätze jedoch unter der allgemeinen und besonders häufig geäußerten, nahezu „klassischen“ Kritik an angewandten Gleichgewichtsmodellen zusammengefasst werden: Angewandte Gleichgewichtsmodelle des internationalen (Agrar-)Handels haben zumindest für Nicht-Modellkundige den Charakter intransparenter Black-Box-Modelle. Diese Kritik berührt verschiedene wichtige Aspekte: das Problem der asymmetrischen Information (Akerlof 1970) zwischen Anbietern und Nachfragern nach Simulationsergebnissen, das Problem der schwierigen Vergleichbarkeit simulierter Ergebnisse aus unterschiedlichen Studien sowie das Problem der schwierigen Interpretierbarkeit von Simulationsergebnissen hinsichtlich ihrer empirischen Aussagen über die wirtschaftspolitische Realität. Diese Probleme werden nachfolgend erläutert.

\subsubsection{Was determiniert Simulationsergebnisse innerhalb der Black Boxes?}

Während angewandte Gleichgewichtsmodelle in der Vergangenheit aufgrund ihrer technischen Komplexität und ihren zeit- und kostenintensiven Anforderungen hinsichtlich der zu Grunde liegende Daten und Parameter nur von verhältnismäßig wenigen Forschergruppen weiterentwickelt und betrieben werden konnten, haben in jüngerer Zeit benutzerfreundliche Innovationen besonders die Anwendung existierender Modellansätze erheblich vereinfacht (Hertel 1997). Benutzerfreundliche Bedienung, größere Rechnerkapazität und die in vielen Modellen verwendete GTAP-Datenbank haben nicht nur zu einer ausgesprochen großen Zahl an Publikationen geführt, welche sich mit Fragen des Welt(agrar)handels auseinandersetzen, sondern rufen auch immer neue Ko- 
operationsmöglichkeiten mit Modellierungsforschern aus dem Bereich der Natur- und Geowissenschaften sowie alternativer Agrarsektormodellansätze (Lineare Programmierung, Multi-Agentensysteme) auf den Plan (Bertelsmeier, Kleinhanss und Offermann 2003).

Die Durchführung von Simulationsexperimenten erfordert dabei noch immer Spezialkenntnisse, welche weder als Standardrepertoire einer ökonomischen noch irgendeiner anderen akademischen Ausbildung angesehen werden können. Bevor Simulationsexperimente anhand angewandter Gleichgewichtsmodelle erstellt und veröffentlicht werden können, ergeben sich demzufolge zunächst Eintrittskosten in Form einer notwendigen Spezialisierung. „Modellierer" (synonym zu „Anbietern von Simulationsergebnissen“, „Modellexperten“, „Modellierungsforschern") haben diese Spezialkenntnisse erworben. Im Gegenzug bildet sich eine Informationsasymmetrie gegenüber all denjenigen, die diese Spezialisierung nicht geleistet haben, aber grundsätzlich auf Ergebnisse angewandter Handelsmodelle zurückgreifen möchten. Im Falle geplanter Änderungen der Handelspolitik sind dies insbesondere Mitglieder der Handelsadministrationen und politische Entscheidungsträger, aber evtl. auch Wissenschaftler, die Simulationsergebnisse als Input für weitere Forschungen verwenden. Diese heterogene Gruppe Nicht-Modellkundiger bildet somit die „Nachfrager nach Simulationsergebnissen“ (nachfolgend synonym zu „NichtModellierern").

Innerhalb der (agrar)ökonomischen Literatur haben sich verschiedene Ansätze zur teilweisen Öffnung der Black Boxes und zur Beseitigung von Informationsasymmetrien zwischen Anbietern von und Nachfragern nach Simulationsergebnissen etabliert: vergleichende Simulationen mit ausgewählten Modellspezifikationen ( $=$ Sensitivitätsanalysen im allgemeinen Sinne) und Simulation eines bestimmten Politikszenarios unter Verwendung alternativer Parameter und Politikvariablen (= Sensitivitätsanalyse gemäß gegenwärtig meist üblicher Praxis).

Dabei sind vergleichende Simulationen mit unterschiedlichen Modellspezifikationen grundsätzlich nicht von Sensitivitätsanalysen zu trennen. Im Bereich der Literatur angewandter Gleichgewichtsmodelle wird der Begriff der Sensitivitätsanalyse aber in erster Linie auf Variationen von Parametern und Politikvariablen bezogen, während beispielsweise die Simulationsexperimente von Gohin und Moschini (2005) zur Auswirkung unterschiedlich restriktiver Closures innerhalb des GTAP-Modells nicht als Sensitivitätsanalyse bezeichnet werden.

Beide Vorgehensweisen, um die Sensitivität von Simulationsergebnissen hinsichtlich bestimmter Spezifikationen des Simulationsaufbaus zu untersuchen, sind innerhalb der gleichgewichtsmodellbasierten Literatur weit verbreitet. 
Im Hinblick auf Szenarien, welche Veränderungen der Handelspolitik untersuchen, ist es inzwischen sogar üblich, Sensitivitätsanalysen der simulierten Politikänderungen sowie verwendeter exogener Parameter durchzuführen und die Ergebnisse dieser Sensitivitätsanalysen gemeinsam mit den simulierten Effekten zu veröffentlichen. Solche Sensitivitätsanalysen können für das jeweilige Modellkonzept zeigen, durch welche Annahmen Simulationsergebnisse tatsächlich am stärksten beeinflusst werden bzw. welche Parameter eventuell für die Größenordnung der Ergebnisse in ihrer Ausprägung vergleichsweise unerheblich sind. Sensitivitätsanalysen erfordern es, eine Auswahl an $k$ Modellkomponenten $(k=1,2, \ldots, K ; K=$ Zahl exogener Variablen innerhalb des Modells) zu treffen und für diese $k$ Komponenten jeweils zu untersuchende Intervalle zu spezifizieren, innerhalb welcher die Robustheit der Simulationsergebnisse evaluiert werden soll (Arndt 1996, Banks 1998, Kleijnen, Sanchez, Lucas und Cioppa 2005).

Im Falle globaler allgemeiner Gleichgewichtsmodelle stellt die simultane Variation mehrerer Parameter innerhalb ausgewählter Intervalle z.T. erhebliche Anforderungen an die Rechnerkapazität, da das gleiche Modell in der Regel hunderte oder tausende Male gelöst werden müsste, um über alle möglichen Kombinationen der zu untersuchenden Parameter Informationen zu generieren. Das Standard-GTAP-Modell bietet eine Funktion zur systematischen Sensitivitätsanalyse (Arndt 1996), welche auf einer approximativen Simulation des Modellverhaltens basiert. Es wird dabei unterstellt, dass das Verhalten des Modells durch ein Polynom dritten Grades näherungsweise abgebildet werden kann, sodass es für eine Sensitivitätsanalyse nicht notwendig ist, das Modell sehr oft lösen zu müssen. Arndt (1996) macht aber deutlich, dass dieses Verfahren nicht in allen Fällen eine hinreichend genaue Approximation für das Verhalten des GTAP-Modells bedeutet und ferner restriktive Annahmen über die Form der Verteilungsfunktion der exogenen Parameter getroffen werden müssen (Arndt 1996). Eine ausführliche Darstellung der Durchführung und Auswertung einer solchen Sensitivitätsanalyse mit dem Systematic Sensitivity Analysis Tool von GTAP findet sich bei Brockmeier (2003).

Sensitivitätsanalysen kommt eine wichtige Bedeutung hinsichtlich der Validierung simulierter Ergebnisse und hinsichtlich eines besseren Verständnisses der Interaktionseffekte innerhalb eines bestimmten Modellkonzepts und Simulationsexperiments zu. Herkömmliche Sensitivitätsanalysen sind jedoch kaum in der Lage, zu einem besseren Verständnis simulierter Ergebnisse über Modellansätze hinweg beizutragen, da sie auf vergleichsweise wenige, meist willkürlich für eine Sensitivitätsanalyse ausgewählte Parameter beschränkt bleiben.

Hinzu kommt, dass es keinen einheitlichen Standard für die Durchführung von Sensitivitätsanalysen gibt und demzufolge kaum Anreize für Modellierer 
bestehen dürften, kritische Sensitivitätsbereiche explizit auszuweisen, sofern diese einer evtl. erwünschten inhaltlichen Aussage entgegen stehen (Hess und von Cramon-Taubadel 2006).

Sensitivitätsanalysen im allgemeinen Sinn weisen diesbezüglich keine grundsätzlich anderen Eigenschaften auf: Viele Autoren zeigen anhand eines bestimmten Modellansatzes, wie sich alternative Modellspezifikationen (beispielsweise konstante Skalenerträge, CRTS, versus steigende Skalenerträge, IRTS) auf die zugehörigen Simulationsergebnisse auswirken (z.B.: Rattsø 1982, Decaluwé, Martens und Monette 1988, Banse und Tangermann 1996, Harrison, Rutherford und Tarr 1997, Xu 2002, Frohberg und Winter 2004, Gohin und Moschini 2005). Aspekte, die gewöhnlich zwischen Simulationsexperimenten unterschiedlicher Studien variieren, wie etwa Datengrundlage, Parameter, etc. werden im Falle solcher vergleichender Experimente meist konstant gehalten.

Hinsichtlich ihrer Eigenschaft, Transparenz zu verbessern, können vergleichende Simulationen und Sensitivitätsanalysen Einblicke in die Funktionsweise und den grundsätzlichen Aufbau alternativer Modellansätze innerhalb eines Modellkonzepts gewähren. Solange solche vergleichenden Experimente jedoch nur innerhalb eines bestimmten Modellierungskonzepts bleiben, sind der Übertragbarkeit der Ergebnisse auf andere Modelle meist enge Grenzen gesetzt.

Der qualitative Vergleich unterschiedlicher Modellierungskonzepte (van Tongeren, van Meijl und Surry 2001) oder unterschiedlicher Modellierungsansätze (Robinson 1989) stellt zwar keine Sensitivitätsanalyse im eigentlichen Sinne dar, geht aber meist auf Erfahrungen der Autoren mit unterschiedlichen Modellierungskonzepten und Modellspezifikationen zurück. Solche überblickartigen Zusammenstellungen sind meist besonders hilfreich für Modellierer, etwa um Modellkonzepte mit alternativen Modellspezifikationen ausfindig zu machen. Anhand einer allgemeinen Beschreibung typischer Modellspezifikationen ist es allerdings meist nicht möglich, verlässliche Rückschlüsse auf das Zustandekommen von Simulationsergebnissen innerhalb einer bestimmten Studie ziehen oder Ergebnisse über Studien hinweg miteinander vergleichen zu können, weshalb qualitative Modellvergleiche für Nicht-Modellierer nur eingeschränkt Transparenz schaffen können.

Hinsichtlich einer generellen Öffnung der Black Boxes über Modellkonzepte hinweg sind Sensitivitätsanalysen und qualitative Modellvergleiche insgesamt somit nur eingeschränkt in der Lage, Transparenz für die Nachfrageseite nach Simulationsergebnissen allgemein zu verbessern.

Die Black-Box-Kritik verweist aber nicht nur auf das allgemein niedrige Vertrauen der Nachfrager nach Simulationsergebnissen in quantitative Politikfolgenabschätzungen, sondern verlangt aufgrund der kostspieligen Entwicklung angewandter Gleichgewichtsmodelle von einem Public Economist (Giersch 1990) auch, nach dem Grad der Effizienz des Einsatzes wissenschaftlicher 
Ressourcen zu fragen, welche in die Konstruktion und den Betrieb von Modellen mit Black-Box-Charakter einfließen. Der in Bezug auf globale Wohlfahrtsszenarien mitunter geäußerte Vorwurf „... their order of magnitude could be estimated without resorting to the computer in any case" (Taylor und von Arnim 2006) kann nur durch die Öffnung der Black Boxes überprüft werden: Indem die Frage beantwortet wird, welche Modellkomponenten die Simulationsergebnisse maßgeblich beeinflussen, könnten evtl. kostengünstigere Verfahren identifiziert werden, welche hinreichende Approximationen simulierter Ergebnisse ermöglichen. Insbesondere die notwendige exogene Parametrisierung angewandter Gleichgewichtsmodelle steht in diesem Zusammenhang in dem Ruf, Simulationsergebnisse maßgeblich zu determinieren und gleichzeitig inner-halb der meisten Modelle nur bedingt empirisch validiert zu sein (McKitrick 1998). Der folgende Abschnitt geht auf diese Kritik an angewandten Gleichgewichtsmodellen ein.

\subsubsection{Unzureichende empirische Absicherung der Modelle}

Der Versuch, einen bestimmten Aspekt der Wirklichkeit realitätsgetreu abzubilden, erfordert im Falle komplexer Systeme wie das des internationalen Handels mit Agrarprodukten fast zwangsläufig eine immense Fülle an Informationen, d.h. komplexe Systeme sind gekennzeichnet durch eine hohe Zahl an Freiheitsgraden (vgl. z.B. Schulz 2003). Beschreibt man ein komplexes System durch Gleichungen, die für bestimmte Variablen des Interesses gelöst werden, müssen diese Gleichungen identifiziert sein, sodass angewandte Gleichgewichtsmodelle - ebenso wenig wie sonstige ökonomische Simulationsmodelle - nicht ohne empirisch gewonnene und innerhalb des Gleichungssystems exogen spezifizierte Parameter, z.B. Elastizitäten, auskommen (Alston und Hurd 1990, McKitrick 1998, Hertel 1999, Hertel, Hummels, Ivanic und Keeney 2003). Zweifellos determiniert die Qualität solcher exogener Parameter die Qualität der späteren Simulationsergebnisse, wenn auch in einem a priori unbekannten Maß. Dies impliziert, dass die methodischen Herausforderungen bei der ökonometrischen Quantifizierung kausaler Zusammenhänge innerhalb der Sozial- und Wirtschaftswissenschaften auch einen begrenzenden Faktor für die empirische Validierung angewandter Gleichgewichtsmodelle darstellen (Sumner und Tangermann 2002, S. 2039).

Ein weiteres wichtiges Problem stellt die Verwendung aggregierter Protektionsdaten (Zölle) dar. Der Aggregations-Bias ist seit langem bekannt, aber es existieren gegenwärtig kaum Möglichkeiten, diesen innerhalb angewandter Gleichgewichtsmodelle zu kontrollieren. Martin, van der Mensbrugghe und Manole (2003) trennen diesen Aggregations-Bias in ein Durchschnittsproblem und ein Gewichtungsproblem und verweisen damit auf zwei unter- 
schiedliche praktische Probleme bei der Aggregierung von Zöllen als Ursache furr den Aggregations-Bias. Anderson und Neary (2003) sowie Anderson (2006) entwickeln konsistente Aggregationsschemata für Zölle. Pelikan und Brockmeier (2007) vergleichen diese Indizes sowie weitere Aggregationsschemata innerhalb globaler Liberalisierungsszenarien; demnach sind simulierte Wohlfahrtseffekte globaler Liberalisierungsszenarien sehr stark abhängig von der verwendeten Aggregationsmethode. Pelikan und Brockmeier (2007) stellen fest, dass der Mercantilist Trade Restrictiveness Index (MTRI) anteilig höhere Wohlfahrtsgewinne in Agrarsektoren und der Trade Restrictiveness Index (TRI) anteilig höhere Wohlfahrtsgewinne in Industriesektoren ausweist.

Pelikan und Brockmeier (2007) kommen ferner zu dem Schluss, dass der theoretisch konsistente TRI-Aggregationsindex gegenüber dem weithin verwendeten, um Handelsmengen gewichteten Durchschnitt deutlich höhere Simulationsergebnisse ausweist, was evtl. darauf hindeutet, dass simulierte Liberalisierunsgewinne insbesondere durch nicht adäquate Zollaggregationsmethoden unterschätzt werden.

Eine weitere wichtige, wenn auch allgemeine Erkenntnis der ökonomischen Theorie, die insbesondere den Bereich exogen spezifizierter Parameter betrifft, aber selten Eingang in angewandte Gleichgewichtsmodelle findet, ist die sog. „Lucas-Kritik“ (Lucas 1976), wonach ökonomische Subjekte zu erwartende Veränderungen (beispielsweise Veränderungen der Handelspolitik) antizipieren und ihre Verhaltensweisen anpassen. Für Gleichgewichtsmodelle bedeutet dies, dass sich Parameter im Zeitablauf verändern können. Solche dynamischen Prozesse können von den meisten Gleichgewichtsmodellen, wenn überhaupt, nur schwer erfasst werden.

Im Rahmen der praktischen Modellierungsarbeit werden exogene Parameter innerhalb angewandter Gleichgewichtsmodelle vielmehr tendenziell über viele Studien und längere Zeiträume hinweg weitgehend beibehalten und entwickeln sich mitunter im Zeitverlauf $\mathrm{zu}$ modellierungstechnischen Konventionen (beispielsweise die Standardparameter im GTAP-Modell), sodass im Durchschnitt vieler Studien die Abhängigkeit von diesen exogenen Parametern evtl. geringer als gemeinhin angenommen ausfallen könnte.

Vergleicht man jedoch die Ergebnisse identisch erscheinender Simulationsszenarien, fällt meist die große Varianz auf, mit welcher beispielsweise simulierte Wohlfahrtseffekte voneinander abweichen können. Dies könnte ein Hinweis darauf sein, dass exogene Parameter sowie weitere Modellspezifikationen tatsächlich im Durchschnitt vieler Studien einen starken Einfluss auf Simulationsergebnisse ausüben. Der folgende Abschnitt untersucht daher, ob bereits anhand qualitativer Vergleiche von Simulationsergebnissen Aussagen über die Wirkungsweise von Modellkonzepten, Modellansätzen und Modellannahmen auf Simulationsergebnisse getroffen werden können. 


\subsubsection{Sind Simulationsergebnisse unterschiedlicher Black Boxes vergleichbar?}

Einige Publikationen präsentieren vermeintlich vergleichbare Szenarien einer bestimmten Politikoption (,Harbinson Proposal“, „Schweizer Formel“, „Agenda 2000 “, etc.) in Form von Tabellen oder Grafiken (Scollay und Gilbert 2000, Francois 2004, Charlton und Stiglitz 2005, Piermartini und Teh 2005, Bouët und Krasniqi 2006) und nicht selten streuen die publizierten Wohlfahrts- und Einkommenseffekte über Modellansätze hinweg mit einer großen Varianz.

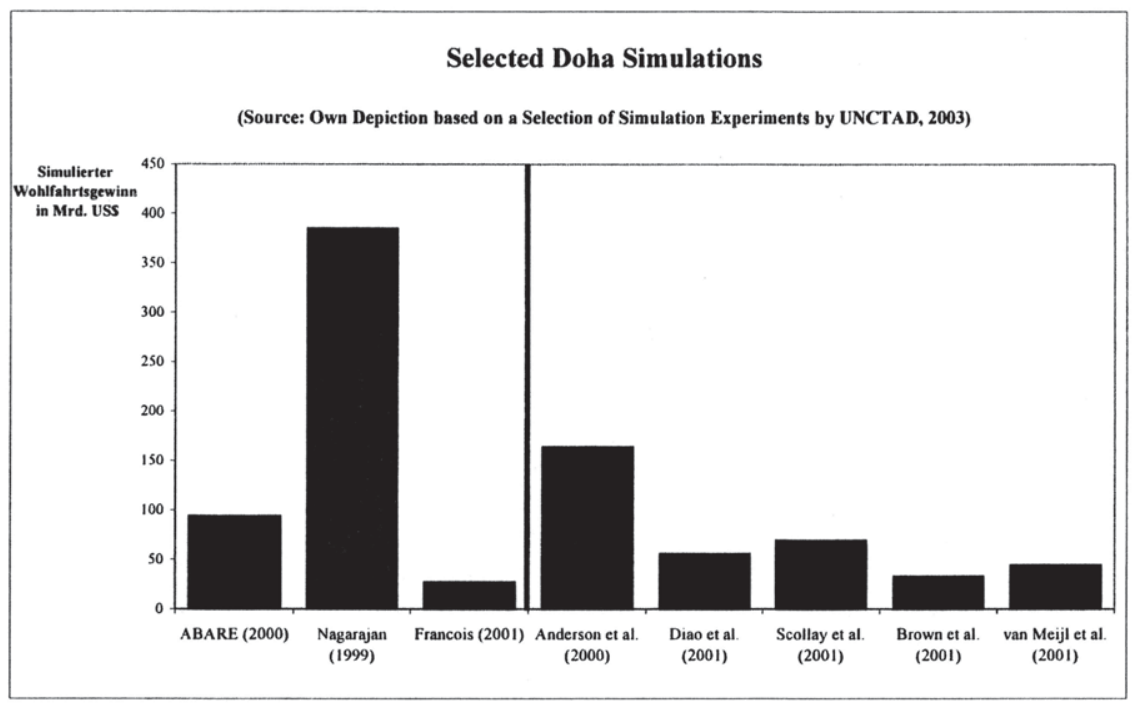

Abbildung 1: Simulierte Weltwohlfahrtseffekte gemäß einer Literaturzusammenstellung von UNCTAD (2003).

Abbildung 1 stellt die in der Übersicht von UNCTAD (2003) erwähnten Simulationsergebnisse verschiedener Studien grafisch dar (vgl. Hess 2005b); Abbildung 1 suggeriert, dass simulierte Effekte einer Veränderung der Weltwohlfahrt sowohl für Simulationsexperimente, die eine 100-prozentige Liberalisierung der Weltagrarmärkte vornehmen, als auch für Experimente mit einer 50prozentigen Liberalisierung stark schwanken. Darüber hinaus ist nicht eindeutig zu erkennen, dass die simulierten Wohlfahrtseffekte einer 100-prozentigen Liberalisierung größer ausfallen als diejenigen einer 50-prozentigen Reduktion aller Agrarzölle weltweit. Insbesondere Nachfrager nach Simulationsmodellen werden durch solche Ergebnisvergleiche stark verunsichert, was den antizipier- 
ten Wert der durchschnittlichen Simulationsstudie in ineffizienter Weise herabsetzt (Asymmetrische Information, Akerlof 1970).

Die Tatsache, dass solche Ergebnisüberblicke während der Doha-Runde wiederholt (Francois 2004, Charlton und Stiglitz 2005, Piermartini und Teh 2005, Bouët und Krasniqi 2006) erstellt wurden, unterstreicht, wie weit der Anspruch und auch die Notwendigkeit im Bereich der quantitativen Politikfolgenabschätzung gegeben sind, numerische Ergebnisse von Simulationsmodellen als quantitative Prognosen zu betrachten und in den Rang von Quasi-Messungen zu erheben. Unabhängig davon, ob man von ausgewiesenen Simulationsergebnissen unterschiedlicher Modellansätze überhaupt erwarten kann, dass sie einem quantitativen Vergleich untereinander standhalten sollten, wird dieser Anspruch offenbar häufig an sie herangetragen.

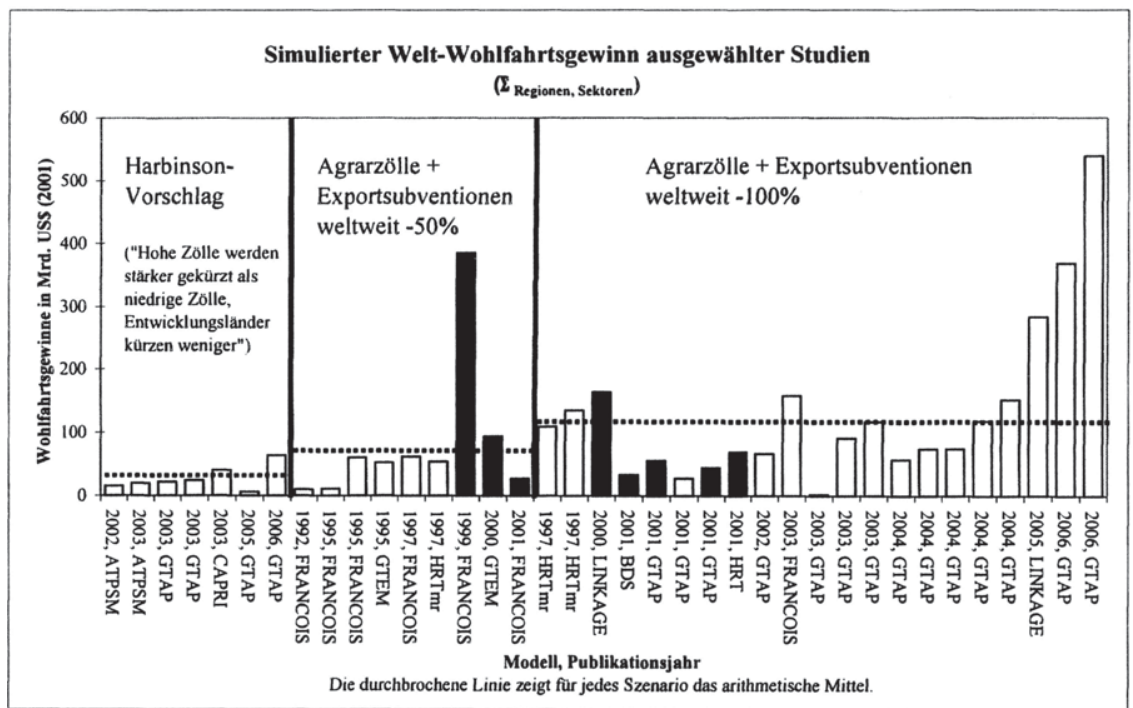

Abbildung 2: Simulierte Weltwohlfahrtseffekte eines repräsentativen Literatursamples. Die schwarz hinterlegten Säulen identifizieren diejenigen Studien, welche auch in Abbildung 1 vorkommen.

Quelle: Eigene Zusammenstellung. Eine Liste der abgebildeten Publikationen ist auf Anfrage erhältlich.

Tabellarische oder grafische Vergleiche simulierter Ergebnisse (vgl. Abbildungen 1 und 2) setzen an dieser Stelle an und behandeln die den Simulationen zu Grunde liegenden Modelle zunächst als Black Boxes. Darstellungen wie in den Abbildungen 1 und 2 verleiten jedoch aufgrund der überwiegend unerklärten Varianz zu letztlich empirisch unbewiesenen Erklärungsansätzen 
hinsichtlich der Wirkungsweise einzelner Modellansätze. Die folgende Anmerkung von Harrison, Rutherford und Tarr (1997) verdeutlicht, dass auch unter Modellierern die durchschnittliche Korrelation bestimmter Modellansätze mit simulierten Ergebnissen über Modellkonzepte hinweg keineswegs immer unstrittig ist, weshalb plausible und korrekte Erklärungen für divergierende Ergebnisse nicht vorausgesetzt werden können:,,The most striking feature of these results [from our model] is, how similar they [= the IRTS, increasing returns to scale] are to the CRTS [= constant returns to scale] version, contrary to the folklore in the CGE literature that IRTS always generates much larger gains due to rationalization gains" (Harrison, Rutherford und Tarr 1997, S. 1420).

Eine Abbildung ähnlich Abbildung 1 birgt also die Gefahr, die tatsächliche Komplexität eines Simulationsexperiments zu sehr zu vereinfachen. Dies liegt teilweise auch an der heterogenen Darstellung von Simulationsergebnissen und den zugehörigen Simulationsexperimenten innerhalb der Literatur: ,....Researchers seem to have found no standard way to report projections in ways that allow for convenient cross-mode comparisons" (Sumner und Tangermann 2002, S. 2037; vgl. auch Hess 2005a).

Abbildung 2 macht deutlich, dass eine breitere und möglicherweise repräsentativere Auswahl von eventuell vergleichbaren Simulationsergebnissen eine von Abbildung 1 stark abweichende Aussage beinhaltet. Anhand einer grafischen Darstellung ähnlich Abbildung 1, die ebenfalls auf einem nicht repräsentativen Literatursample beruht, identifizieren Bouët und Krasniqi (2006) vermeintlich einen Zusammenhang zwischen dem Publikationsjahr einer Studie und im Zeitverlauf fallenden simulierten Ergebnissen für weltweite Wohlfahrtsänderungen (,Trade Pessimism", vgl. auch Ackerman 2005). Dieser Zusammenhang könnte anhand Abbildung 1 eventuell gestützt werden, müsste anhand von Abbildung 2 aber eher als "Trade Optimism " bezeichnet werden.

Aufgrund dieser Unstimmigkeiten werden Abbildungen ähnlich den Abbildungen 1 und 2 insgesamt wahrscheinlich kaum zu aufschlussreichen Vergleichen unterschiedlicher Studien beitragen können. Gleichzeitig wird anhand solcher Darstellungen nicht deutlich, welche Annahmen letztlich zum Zustandekommen simulierter Ergebnisse entscheidend beigetragen haben. Selbst wenn die Varianz innerhalb simulierter Wohlfahrtseffekte über Studien hinweg insgesamt toleriert werden könnte, stellt sich dennoch die Frage, welche Aussage solche simulierten Wohlfahrtseffekte für eine politische Handlungsoption im Durchschnitt vieler Studien beinhalten. Das folgende Kapitel 2.3.4 geht dieser Frage nach. 


\subsection{Theorie und Wirklichkeit: Die problematische Interpretation von Simulationsergebnissen}

Angewandte Gleichgewichtsmodelle weisen die Resultate von Simulationsexperimenten in Form numerischer Ergebnisse (z.B. Wohlfahrtsveränderungen) aus, die sich als Folge einer bestimmten simulierten Politikänderung ergeben. Dabei muss wie in jedem wissenschaftlichen Modell davon ausgegangen werden, dass erstens die abgebildete Politik lediglich eine unvollkommene Repräsentation der Wirklichkeit darstellt und zweitens der Modellkontext, innerhalb dessen diese Politikänderung simuliert wird, nur eine unvollkommene Abbildung der Realität darstellt: „Theory is vital, but it is not enough. Theory does not 'say' - as is often asserted by the ill-informed or the badly taught - that 'free trade is best'. It says that, given certain assumptions, it is 'best"' (Corden, 1974; S. 8 Hervorhebung im Original).

Sowohl bei der Abbildung einer Politik als auch bei der Repräsentation des ökonomischen Kontextes, innerhalb dessen diese Politik simuliert werden soll, müssen Annahmen getroffen werden. Um Simulationsergebnisse als Informationsquelle für politische bzw. ökonomische Prozesse verwenden zu können, ist demzufolge ein Verständnis der Abweichungen dieser Modellannahmen von den Gegebenheiten der Realität unerlässlich (Sumner und Tangermann 2002).

Idealerweise stellen Modellannahmen kein oder nur ein geringfügig verzerrtes Abbild der Realität dar, was jedoch im Falle angewandter Gleichgewichtsmodelle nicht immer der Fall ist, da bereits die Handelstheorie einige vergleichsweise restriktive Annahmen enthält, die eine Übertragung auf politische Gegebenheiten der Realität nur selten unmittelbar erlauben (Corden 1974). Eine Bewertung der Wirklichkeit anhand dieser Ergebnisse kann nur unter Einbeziehung des experimentellen Aufbaus geschehen. Erst die Kenntnis dieser Kausalität erlaubt eine sinnvolle Interpretation von Simulationsergebnissen.

Zhang (2006) betont beispielsweise, dass durch die Armington-Annahme in angewandten Handelsmodellen Interaktionseffekte zwischen allokativen Wohlfahrtseffekten und ToT-Effekten entstehen, welche schwer vorhersagbar sind und in manchen Fällen zu schwer interpretieren Ergebnissen führen, d.h. die Kausalität beim Zustandekommen der simulierten Wohlfahrtseffekte ist sehr komplex und je nach Konstellation von Nettohandelsposition, relativem Protektionsniveau eines Landes und Struktur der Absatzmärkte verschieden. Zhang (2006) präsentiert eine schematische Interpretation des Effektes der ArmingtonAnnahme in einem multiregionalen Kontext. Er nimmt Bezug auf die Arbeit von Brown (1987) und merkt an, dass die Interaktionseffekte, welche durch die 
Armington-Annahme in ein Modell eingeführt werden, bislang keineswegs erschöpfend verstanden sind.

Durch meist nichtlineare funktionale Zusammenhänge innerhalb der Modelle interagieren die vielen endogenen und exogenen Variablen innerhalb angewandter Gleichgewichtsmodelle insgesamt offenbar häufig in einer Weise, die eine eindeutige Interpretation der Simulationsergebnisse selbst bei profunder Kenntnis der Handelstheorie erschwert: „The models... are ... complex enough to defy comprehension of what drives the results..." (Sumner und Tangermann 2002, S. 2039). Eine korrekte Interpretation simulierter Ergebnisse ist jedoch Voraussetzung für die eigentliche Evaluation und Bewertung einer geplanten Politikänderung anhand eines Simulationsexperiments (Devarajan und Robinson 2002, Sumner und Tangermann 2002).

Die Entwicklung von Methoden, welche dieses Verständnis der allgemeinen Kausalität zwischen Simulationsergebnissen und Modellspezifikationen innerhalb angewandter Gleichgewichtsmodelle verbessern, kann somit möglicherweise dazu beitragen, dass innerhalb politischer Entscheidungsfindungsprozesse anhand von Simulationsergebnissen leichter Rückschlüsse auf die wirtschaftspolitische Realität angestellt werden können. Durch Fokussierung auf die Rolle besonders wichtiger Annahmen innerhalb von angewandten Gleichgewichtsmodellen könnten zudem Diskussionen zwischen Modellierern und Nicht-Modellierern hinsichtlich einer Bewertung der Größenordnung simulierter Ergebnisse vereinfacht werden.

Im folgenden Kapitel wird die Methode der Meta-Analyse auf angewandte Gleichgewichtsmodelle übertragen, um solche über Modellansätze hinweg besonders wichtigen Modellannahmen empirisch identifizieren und deren Rolle für die Ergebnisse simulationsmodellbasierter Studien während der Doha-Runde quantifizieren zu können. 
Sebastian Hess - 978-3-631-75694-2

Downloaded from PubFactory at 01/11/2019 02:52:17AM

via free access 


\section{Methode und Materialien: Meta-Analyse}

Divergierende Ergebnisse zu gleichen oder ähnlichen Fragestellungen sind in der Wissenschaft nicht ungewöhnlich; die Abhängigkeit komplexer Simulationsmodelle von exogenen Daten sowie die Dominanz a priori nicht antizipierter Interaktionseffekte zwischen Experiment und Modellkontext ist keineswegs auf angewandte Gleichgewichtsmodelle beschränkt, sondern stellt eine zentrale Herausforderung für die meisten angewandten Simulationsmodelle im ökonomischen, naturwissenschaftlichen und technischen Bereich dar (Banks 1998). Für beide Probleme haben sich methodische Ansätze etabliert, die allerdings bisher kaum Anwendung im Bereich angewandter Gleichgewichtsmodelle erfahren haben: Meta-Analysen und Meta-Modellierung. Letztere kann als MetaAnalyse auf Basis generierter Simulationsdaten über ein oder mehrere Modelle hinweg verstanden werden, weshalb Meta-Modellierung auch als eine besondere Form der Meta-Analyse angesehen werden kann. In diesem Kapitel wird die Methode der Meta-Analyse erläutert und Möglichkeiten, diese auf angewandte Gleichgewichtsmodelle zu übertragen, werden erörtert.

\subsection{Meta-Analyse als quantitativer Ergebnisvergleich}

Grundsätzlich werden bei Meta-Analysen nicht die Forschungsgegenstände selbst (z.B. Wohlfahrtswirkung von Agrarpolitik), sondern die bereits publizierten empirischen Effekte aus mehreren wissenschaftlichen Studien zu einem bestimmten Thema einer eingehenden, quantitativen Analyse unterzogen. Ein meta-analytischer Ansatz unterstellt, dass die Varianz innerhalb voneinander abweichender Ergebnisse aus Studien zu einer sehr ähnlichen oder sogar identischen Forschungsfrage nur teilweise zufälliger Natur ist. Vielmehr sucht eine Meta-Analyse nach Informationen, welche einen Teil dieser Varianz zwischen den Ergebnissen erklären können (Stanley 2001).

Ursprünglich wurde Meta-Analyse zum Zweck der Forschungssynthese im Bereich der Naturwissenschaften entwickelt (eine Geschichte der Methode findet sich z.B. bei Cooper 1998). Ziel ist es, quantitative Informationen unterschiedlicher Studien zu einer ähnlichen oder gleichen Fragestellung effektiver nutzen zu können (Cooper 1998). Diese Methode betrachtet Studien zu einem bestimmten Thema dabei als einzelne Observation und versucht, den durchschnittlich gemessenen Effekt beispielsweise zur Korrelation zwischen Pestizidrückständen in Nahrungsmitteln und Gesundheitsschäden bei Verbrauchern 
zu ermitteln. Dabei müssen verwendbare Studien meist das Kriterium einer Vergleichsgruppe erfüllen, d.h. alle Studien müssen einen Effekt im Vergleich zu einer Referenzgruppe (z.B. Placebo-Patienten) enthalten, um Vergleichbarkeit untereinander zu ermöglichen (Button und Jongma 1995).

Viele solcher Meta-Analysen verwenden die folgende (Stanley und Jarrell 1989) oder eine ähnliche Definitionen des in einer Studie ausgewiesenen Effekts, beispielsweise als Ergebnis eines Versuchs:

$$
g=\left(\mu_{e}-\mu_{k}\right) / \sigma
$$

Bei $\mu_{e}$ und handelt es sich um den Mittelwert einer Versuchsgruppe, welche einem bestimmten Versuchseinfluss ausgesetzt wurde; $\mu_{k}$ ist der Mittelwert der zugehörigen Kontrollgruppe. Die Differenz dieser Werte wird durch die Standardabweichung der Kontrollgruppe $(\sigma)$ geteilt. Stanley und Jarrell (1989) betonen in diesem Zusammenhang die Bedeutung eines gemeinsamen und über Studien hinweg vergleichbaren Maßes für die Durchführbarkeit einer MetaAnalyse; dieses Maß besteht häufig in dieser Standardabweichung $(\sigma)$ der Kontrollgruppe.

Meta-Analyse zur Forschungssynthese hat während der vergangenen zwei Jahrzehnte vornehmlich im Bereich der Medizin, Pharmazie, Psychologie und Pädagogik weite Verbreitung gefunden (Cooper 1998). Über den Aspekt der Ergebnissynthese hinaus werden aber auch in vielen Meta-Analysen verfuggare Informationen aus den jeweiligen Studien und über die jeweiligen Studien zusammengeführt, um Erkenntnisse darüber zu gewinnen, wie Ergebnisse durch bestimmte Methoden beeinflusst werden. Dieser evaluatorische Ansatz gewinnt besonders in der Ökonomie und verwandten Disziplinen zunehmend an Bedeutung (Stanley und Jarrell 1989) und erweitert die ursprüngliche MetaAnalyse mit ihrer starken Fokussierung auf der Ergebnissynthese um den Aspekt der Erklärung von Unterschieden zwischen den einzelnen Studien.

Kritiker der Meta-Analyse bringen besonders folgende Argumente vor, die zur Orientierung darüber dienen können, ob die Anwendung der Methode überhaupt im jeweiligen Fall sinnvoll erscheint:

- „Junk in- junk out": Werden Ergebnisse „richtiger“ und „falscher“ methodischer Durchführung zu einer Fragestellung kombiniert, verwischt die Meta-Analyse lediglich die gemessenen Effekte der besseren Studien zu Gunsten von Effekten, welche eigentlich keine Berücksichtigung erfahren sollten (Wachter 1988, Eysenck 1995, van den Bergh, Button, Nijkamp und Pepping 1997).

- „Comparing Apples and Oranges“: Sind die kombiniert betrachteten Studien in ihrem methodischen Ansatz sehr unterschiedlich, kann eine Meta-Regression über die gemessenen Effekte kaum sinnvolle Infor- 
mationen enthalten, da viele wissenschaftliche Methoden ihre optimale Wirkungsweise nur unter sehr spezifischen Umständen entfalten (Wachter 1988, Eysenck 1995).

- „Publication Bias“: In einigen wissenschaftlichen Disziplinen, z.B. der Ökonomie, tendieren Journale zur Veröffentlichung von Studien mit signifikanten Ergebnissen oder bevorzugen Studien, welche etablierte Paradigmen affirmieren (Button und Jongma 1995, Stanley 2005). Nicht veröffentlichte Ergebnisse können für eine Meta-Analyse daher sehr wertvolle Informationen enthalten, sind aber kaum zugänglich (Button und Jongma 1995, Stanley 2005). Ebenfalls könnte eine Pionierstudie zu einem bestimmten Thema ,verzerrt" sein und eine Reihe anderer Studien dieser Art nach sich ziehen, was die quantitativen Effekte innerhalb einer Meta-Analyse ebenfalls verzerren würde (van den Bergh et al. 1997, Stanley 2005).

Um die Methode der Meta-Analyse sinnvoll anwenden zu können, müssen die verwendeten Studien zu einem bestimmten Effekt methodisch einem vergleichbaren Ansatz folgen, wissenschaftlich von vergleichbarer Qualität sein und innerhalb der publizierten Literatur darüber hinaus so zahlreich sein, dass verzerrende Effekte sich möglichst stochastisch aufheben, d.h. Meta-Analysen sind allgemein nicht sinnvoll im Bereich junger Forschungsgebiete mit einem hohen Anteil von Studien, welche methodische Innovationen $\mathrm{zu}$ einer bestimmten Fragestellung präsentieren. Stattdessen kennzeichnet das Erscheinen von MetaAnalysen häufig, dass eine Forschungsfrage bereits ausgiebig, wenn auch noch nicht erschöpfend, untersucht wurde (Florax 2002).

Für eine Meta-Analyse angewandter Gleichgewichtsmodelle ist der „Junk in- junk out"-Vorwurf wahrscheinlich von untergeordneter Bedeutung, da quantitative Simulationen auf Basis berechenbarer Gleichgewichtsmodelle grundsätzlich hypothetische Szenarien erstellen. Die Qualität dieser Prognosen hinsichtlich eines in der Realität beobachteten Effektes ist aber nicht Gegenstand dieser Meta-Analyse. "Schlechte“ Simulationsstudien können sich von „guten“ Studien also lediglich durch technisch fehlerhafte Verwendung des Modells unterscheiden. Die mögliche Existenz solcher Studien kann aber a priori keinen Grund darstellen, eine solche Analyse nicht durchzufuhren; vielmehr können fehlerhafte Studien, sofern sie unbemerkt Eingang in die Literatur gefunden haben, evtl. im Rahmen der Durchfuhrung einer Meta-Analyse identifiziert werden. Sollten viele Studien kleinere Fehler enthalten, kann uberdies angenommen werden, dass sich die Fehler im Mittel vieler Studien gegenseitig stochastisch aufheben.

Bei gleichgewichtsmodell-basierten Simulationen handelt es sich zudem um Simulationsexperimente aus vergleichsweise ähnlich aufgebauten Modellierungskonzepten (van Tongeren, van Meijl und Surry 2001). Es werden im 
Rahmen einer Meta-Analyse also nicht „Äpfel mit Birnen“, sondern „Äpfel unterschiedlicher Sorten" miteinander verglichen.

Ein wesentlich größeres Problem als eventuell mangelnde konzeptionelle und qualitative Homogenität stellt für das geplante Forschungsvorhaben die quantitative Vergleichbarkeit der in die Meta-Analyse einbezogenen Studien dar, also beispielsweise die Vergleichbarkeit einer Effektmessung als Veränderung des Bruttoinlandsproduktes $\triangle \mathrm{BIP} /$ Einwohner gegenüber einer Messung als $\triangle B I P$. In vielen Fällen kann jedoch eine näherungsweise Transformation der Ergebnisse auf ein gemeinsames Vergleichsmaß vorgenommen werden (Bsp. $\Delta \mathrm{BIP} /$ Einwohner ${ }^{*}$ Einwohner im Simulationszeitraum $=\Delta B I P$, usw.).

In vielen Meta-Analysen werden anhand eines detaillierten Fragebogens relevante Informationen aus den jeweiligen Studien (im Falle von angewandten Gleichgewichtsmodellen wären dies simulierte Wohlfahrts- und Einkommenseffekte, Politikmaßnahmen, etc.) und über die jeweiligen Studien (Charakteristik der Modelle) extrahiert und in einem Meta-Datensatz zusammengefasst. Die erfassten Variablen sollen im Falle der vorliegenden Meta-Analyse durch ein multivariates Regressionsmodell und nicht durch eine Kombination von gemessenen Effekten auf systematische Beziehungen hin untersucht werden (Stanley 2001, Hedges und Olkin 1985, vgl. auch die Verfahrensweise von Thiam, Bravo-Ureta und Rivas 2001). Die geschätzten Koeffizienten werden Aufschluss darüber geben, wie simulierte Ergebnisse auf Basis von Gleichgewichtsmodellen mit Annahmen innerhalb von Gleichgewichtsmodellen korreliert sind, was wiederum Rückschlüsse darüber zulässt, welche Modellkomponenten für welche Ergebnisse entscheidend verantwortlich sind.

\section{2 Ökonometrische Aspekte der Meta-Analyse}

Die von Stanley und Jarrell (1989) für den Bereich ökonomischer Studien eingeführte Meta-Analyse verwendet als Meta-Modell ein lineares Regressionsmodell (Standard Regressionsmodell mit Kleinstquadratschätzer, OLS). Dies bedeutet, dass die Ergebnisse (ökonometrischer) Studien innerhalb des Meta-Datensatzes durch ein solches lineares Meta-Regressionsmodell approximiert und die Varianz innerhalb der abhängigen Variable über Studien hinweg erklärt wird. Genau wie angewandte Gleichgewichtsmodelle beinhalten jedoch auch lineare Regressionsmodelle Annahmen, durch welche ihre Aussagekraft entscheidend determiniert wird. Dieses Kapitel diskutiert die Relevanz dieser Annahmen sowie mögliche Abweichungen von ihnen hinsichtlich der ökonometrischen Umsetzung einer Meta-Analyse angewandter Gleichgewichtsmodelle und fasst 
die wichtigsten Verletzungen und Erweiterungen der Grundannahmen des linearen Regressionsmodells zusammen.

\subsubsection{Meta-Analyse und lineare Regressionsmodelle}

Im Fall der vorliegenden Meta-Analyse von Simulationsergebnissen angewandter Gleichgewichtsmodelle existiert, wie für ökonomische Studien meist zu erwarten, keine Vergleichsgruppe (siehe Gleichung 3.1). Stanley und Jarrell (1989) fassen zusammen, welche Besonderheiten sich in solchen Fällen für Meta-Analysen ergeben: Eine Meta-Analyse ökonomischer Literatur untersucht demnach meist Regressionsergebnisse (geschätzte Koeffizienten) als zu erklärende Variablen, da empirische Ergebnisse in der Ökonomie häufig - nicht immer - anhand von Regressionsmodellen untersucht werden. Das StandardRegressionsmodell (OLS) hat dabei die bekannte Form:

$$
Y=X \beta+\epsilon,
$$

wobei Y ein $(n \times 1)$-Vektor mit Beobachtungen der zu erklärenden Variable darstellt und $\mathrm{X}$ eine $(\mathrm{n} \times \mathrm{m})$-Matrix der erklärenden Variablen bildet. Die zu schätzenden Regressionskoeffizienten sind $\operatorname{der}(\mathrm{m} \times 1)$-Vektor $\beta, \epsilon$ ist der stochastische Fehlerterm, für den angenommen wird, dass $\epsilon$ den Erwartungswert $E(\epsilon)=0$ hat sowie dass die Zufallsvariablen stochastisch unabhängig von einander sind und eine homogene Varianz aufweisen. Geht man davon aus, dass die verwendeten Daten die Struktur $\left(y_{i}, x_{\mathrm{i}}\right)$ mit $i=1,2, \ldots, N ; N=$ Zahl der Observationen haben, dann bedeutet dies für die Struktur des Fehlerterms (I bezeichnet die Einheitsmatrix):

$$
\operatorname{Cov}(\epsilon)=\sigma^{2} I_{n}
$$

Entsprechend fuhren Stanley und Jarrell (1989) das folgende allgemeine MetaRegressionsmodell für den qualitativen Vergleich empirischer, ökonomischer Literatur ein:

$$
b_{i}=\beta+\sum \alpha_{k} Z_{i k}+e_{i} \quad i=1,2, \ldots, L
$$

Die abhängige Variable dieser Regression $\left(b_{j}\right)$ ist dabei der ausgewiesene Koeffizient einer über $j$ Studien hinweg zu vergleichenden Variable der $j$-ten Publikation innerhalb des Literatursamples mit $L$ Publikationen. Von Interesse ist dabei insbesondere der Wert des Parameters $\beta$ in diesem Modell, welcher als der über Studien hinweg gemessene durchschnittliche Effekte von $(b$,$) inter-$ pretiert wird. $Z_{k}$ ist eine unabhängige Meta-Variable für $k=1,2, \ldots, K$ Meta- 
Variablen, wobei $K=$ Anzahl der erklärenden Meta-Variablen in einem MetaRegressionsmodell ist. Die Variablen $Z$ bilden relevante Charakteristika empirischer Studien innerhalb der $\mathrm{zu}$ untersuchenden Literatur ab und kontrollieren für ihre systematische Variation; $\alpha$ ist der Meta-Regressionskoeffizient, welcher den Effekt einer bestimmten Charakteristik $Z$ auf die Ergebnisse quantifiziert, und $e$ ist der stochastische Fehlerterm innerhalb der Meta-Regression. Da es sich in den meisten ökonomischen Meta-Analysen, wie bereits erwähnt, um eine Untersuchung geschätzter Regressionskoeffizienten handelt, gehen Stanley und Jarrell (1989) davon aus, dass auch der Fehlerterm $e$ mit Ausnahme nicht konstanter Varianz der Fehlerterme die im Zusammenhang mit dem OLS-Schätzer angenommenen Eigenschaften (Normalverteilung, homogene Varianz, Unabhängigkeit) haben wird.

Im Fall einer Meta-Analyse angewandter Gleichgewichtsmodelle sind weder Voraussetzungen für eine Meta-Analyse gemäß Gleichung (3.1) (Suche nach dem durchschnittlichen Effekt) noch Voraussetzungen typischer ökonometrischer Meta-Analysen (Regressionskoeffizienten bzw. Standardabweichung als vergleichbare Größe über Publikationen hinweg) gegeben. Simulierte Änderungen endogener Modellvariablen als Folge von Politikänderungen tragen zwar durchaus Merkmale eines gemessenen Effektes, da sie stets als Änderungen gegenüber einer Baseline oder gegenüber einem Referenzszenario (z.B. Freihandel) ausgewiesen werden. Die Ausgestaltung dieser Referenzszenarios ist aber bei multiregionalen und multisektoralen Modellen, welche z.T. mehrere simultane Politikänderungen gleichzeitig untersuchen und sich üblicherweise auf Daten aus unterschiedlichen Quellen beziehen, so unterschiedlich, dass eine Identifizierung eines gemeinsamen Effektes wie beispielsweise der Standardabweichung der Vergleichsgruppe nicht möglich ist, sofern nicht für viele verschiedene Einflussgrößen gleichzeitig (multivariat) kontrolliert wird. Die Vorgehensweise zur Erhebung erklärender Variablen im Rahmen einer MetaAnalyse angewandter Gleichgewichtsmodelle muss daher diesen Bedingungen Rechnung tragen.

Hinsichtlich der Umsetzung einer ökonometrischen Meta-Analyse nach Stanley und Jarrell (1989) kann ebenfalls zunächst keine einheitliche Größe $b_{\mathrm{j}}$ innerhalb einer abhängigen Variable identifiziert werden. Andererseits weisen Simulationsexperimente auf Basis angewandter Gleichgewichtsmodelle wie in Kapitel 2.1 erläutert stets eine typische Struktur auf, welche durch folgenden Zusammenhang charakterisiert ist:

$$
\Delta Y=f(\Delta P+M+D),
$$

Mit: $Y=$ endogene Modellvariable, z.B. Einkommen; dies ist häufig die Zielgröße der (simulierten) Politikänderung $\Delta P ; M=$ Vektor von Modellcharak- 
teristika; $D=$ Ausgangsdaten und Parameter. Dies bedeutet, dass sich simulierte Änderungen endogener Zielgrößen stets als Funktion spezifizierter (,geschockter") Änderungen bestimmter exogener Modellvariablen (z.B. Zölle) sowie eines Vektors relativ zum Referenzszenario konstant gehaltener exogener Modellcharakteristika (Funktionsformen, Daten, etc.) ergeben. Es sollte daher möglich sein, für Unterschiede hinsichtlich der Spezifikation von $Y, \triangle P, M$ und $D$ zumindest approximativ zu kontrollieren, wodurch der Grundgedanke einer Meta-Analyse erfüllt ist.

Das Standard-Regressionsmodell stellt in vielen Meta-Analysen den Ausgangspunkt der ökonometrischen Meta-Modellierung dar. Ein besonderer Vorteil der linearen Regression für Meta-Analysen liegt in der Tatsache, dass dieses Modell in vielen wissenschaftlichen Disziplinen verbreitet ist und daher besonders viele Adressaten einer Meta-Analyse mit einem solchen Modell grundsätzlich vertraut sind. Im Falle angewandter Gleichgewichtsmodelle, die für ihren Black-Box-Charakter berüchtigt sind, ist daher die Wahl eines besonders übersichtlich strukturierten ökonometrischen Meta-Modells vorteilhaft. Dies schränkt jedoch möglicherweise a priori die Möglichkeiten zur ökonometrischen Meta-Modellierung ein. Es muss daher sorgfältig überprüft werden, ob und unter welchen Umständen die ökonometrische Umsetzung eines linearen Regressionsmodells für eine Meta-Analyse angewandter Gleichgewichtsmodelle in Frage kommt. Nachfolgend werden daher typische Erweiterungen und Grenzen des Standard-Regressionsmodells hinsichtlich ihrer möglichen Relevanz für die durchzuführende Meta-Analyse diskutiert. Da es sich bei diesen Vorüberlegungen um verbreitete ökonometrische Phänomene handelt, wird auf die komprimierten Darstellungen von Standardwerken (Rawlings, Pantula und Dickey 1998, Goldberger 1998, Wooldridge 2002, Greene 2003, Gujarati 2003, Kutner, Nachtsheim, Neter und Li 2005) und nur in Einzelfällen auf Originalbeiträge Bezug genommen.

Hinsichtlich der Deklaration von Variablen gilt nachfolgend - soweit nicht anders erläutert, dass $Y$ auch als zu erklärende Meta-Variable interpretiert werden kann und Eigenschaften einer erklärenden Variable $X$ auch für eine MetaVariable $Z$ gelten.

\subsubsection{Multikollinearität}

Die Tatsache, dass angewandte Gleichgewichtsmodelle meist auf Basis eines geschlossenen theoretischen Konzeptes formuliert werden, impliziert bereits, dass viele Modellspezifikationen einander bedingen, was im ökonometrischen Sinne eine mögliche Kollinearität erklärender Variablen bedeutet. Dieses Phänomen wird gemeinhin als „Multikollinearität" bezeichnet, wobei üblicherweise der Grad der vorliegenden Korrelation nicht vollkommen sein muss, um das 
Kriterium der Multikollinearität zu erfüllen. Andererseits existiert außerhalb vollkommener Multikollinearität keine eindeutige Abgrenzung hinsichtlich der Tatsache, wann man von "starker" oder "schwacher" Multikollinearität spricht (Wooldridge 2002, Kutner et al. 2005). Gleichung 3.5 (Gujarati 2003) verdeutlicht das Problem unvollständiger Multikollinearität:

$$
\lambda_{1} X_{1}+\lambda_{2} X_{2}+\ldots+\lambda_{k} X_{k}+v_{i}=0
$$

Mit: $\mathrm{X}=$ erklärende Variablen in einer Regression; $\lambda=$ Kostanten; $\mathrm{v}=$ stochastischer Fehlerterm für $i=1,2, \ldots, \mathrm{n}$ Observationen. Gleichung (3.5) zeigt unvollständige Multikollinearität, da sich die $X_{k}$ Variablen aufgrund des stochastischen Fehlerterms $v_{i}$ nicht vollständig in einander umwandeln lassen, aber einen starken linearen Zusammenhang aufweisen. Die Tatsache, dass erklärende Variablen miteinander korreliert sind, wird, sofern diese Korrelation nicht perfekt ist (d.h. Korrelationskoeffizient $=1$ und $v_{i}=0$ ), innerhalb der Literatur grundsätzlich nicht als Problem für die OLS-Schätzung angesehen; der OLSSchätzer bleibt ein BLUE (Best Linear Unbiased Estimator). Als Konsequenz starker Korrelation der Kovariate ergeben sich jedoch große Konfidenzintervalle (und folglich niedrige t-Werte der zu schätzenden Koeffizienten), was eine präzise Schätzung einzelner Einflussgrößen auf die abhängige Variable schwierig oder unmöglich macht (vgl. z.B. Goldberger 1998, Wooldridge 2002, Kutner et al. 2005).

Im Falle starker Multikollinearität können ferner viele t-Statistiken zwar insignifikant sein, obwohl das Regressionsmodell gleichzeitig einen sehr hohen Determinationskoeffizienten $\left(\mathbf{R}^{2}\right)$ aufweist. Ferner können die OLS-Schätzer und ihre Standardfehler sehr empfindlich für kleinere Veränderungen innerhalb der Daten werden (Gujarati 2003).

Die praktischen Konsequenzen von Multikollinearität werden häufig als äquivalent $\mathrm{zu}$ den praktischen Konsequenzen einer Arbeit mit (zu) wenigen Beobachtungen angesehen (vgl. Diskussionen in Wooldridge 2002, Gujarati 2003, Goldberger 1998). Es existieren einige mehr oder weniger formale Methoden, den Grad der Multikollinearität innerhalb erklärender Variablen zu quantifizieren: Wie bereits erwähnt, ist ein hohes $R^{2}$ bei insgesamt niedrigen tWerten ein augenscheinlicher Hinweis für starke Multikollinearität innerhalb der erklärenden Variablen. Dies kann beispielsweise auch auftreten, wenn viele Interaktionsterme geschätzt werden, was ebenfalls als eine Form der Multikollinearität interpretiert werden kann (Bernhardt und Jung 1979). Im Fall vieler Interaktionsterme verlieren die einzelnen t-Statistiken ebenfalls ihre Aussagekraft und vielmehr sollte die Signifikanz einer Gruppe von Koeffizienten anhand eines F-Tests überprüft werden (Bernhardt und Jung 1979). 
Auch die partielle Korrelation zwischen erklärenden Variablen kann Auskunft über den Grad der Multikollinearität geben. Gujarati (2003) weist darauf hin, dass dies zwar eine hinreichende, aber keine notwendige Bedingung für das Vorhandensein von starker Multikollinearität darstellt. Ein verbreitetes Testverfahren für Multikollinearität ist die Eigenwert-basierte Ermittlung des Konditionsindexes bzw. der Konditionszahl $\kappa$, welche wie folgt definiert ist:

$$
\kappa=\operatorname{Maximum}(\text { Eigenwert }) / \operatorname{Minimum}(\text { Eigenwert })
$$

Als Faustregel gilt, dass ein $\kappa$ zwischen 100 und 1000 mittlere bis starke MultiKollinearität anzeigt und $\kappa$ größer 1000 auf schwere Multikollinearität hinweist (Gujarati 2003).

Mitunter wird auch der Varianz-Inflationsfaktor (VIF) herangezogen. Hier gilt als Faustregel, dass ein VIF größer 10 (tritt auf ab $R^{2}>0.9$ ) auf starke Kollinearität hindeutet (Gujarati 2003). Der VIF ist für zwei erklärende Variablen $\mathrm{X}_{2}$ und $\mathrm{X}_{3}$ definiert als

$$
V I F=1 /\left(1-\left(\text { Korrelationskoeffizient } X_{2}, X_{3}\right)\right)
$$

Das Phänomen der Multikollinearität wird aufgrund der deterministischen Struktur angewandter Gleichgewichtsmodelle bei der praktischen Umsetzung der Meta-Analyse zu erwarten sein. Es muss anhand der genannten Verfahren statistisch überprüft werden, stellt aber kein grundsätzliches Problem für die Verwendung des Ansatzes dar, sofern ein ausreichend „großes“ Sample ermittelt werden kann, welches hinreichend viele Informationen über unterschiedliche Ausprägungen der Kovariate $Z_{\mathrm{k}}$ erfasst (Wooldrige 2002, Gujarati 2003, Goldberger 1998). Hierzu ist die Formulierung voneinander unabhängiger Kategorien dieser erklärenden Meta-Variablen $Z_{\mathrm{k}}$ unerlässlich. Hinweise auf eine zu erwartende Kollinearität können dabei sowohl Modelldokumentationen als auch Zusammenhänge innerhalb der ökonomischen Theorie geben.

\subsubsection{Ansätze zur Varianzreduktion innerhalb der erklärenden Variablen}

Es kann erwartet werden, dass eine Meta-Analyse angewandter Gleichgewichtsmodelle aufgrund der Komplexität dieser Modelle eine Vielzahl an Beobachtungen hervorbringen wird, welche möglicherweise Änderungen der abhängigen Variable erklären können, von welchen aber viele auch einen hohen Grad an Kollinearität aufweisen werden. Sogenannte „dimensionsreduzierende Verfahren" wie beispielsweise die Faktor(en)analyse (unter diesem Oberbegriff ist auch die bekannte Hauptkomponentenanalyse, PCA, einzuordnen) werden üblicherweise verwendet, um mehrere erklärende Variablen anhand ihrer ge- 
meinsamen Varianz zu gruppieren und durch sogenannte „Faktoren“ $\mathrm{zu}$ beschreiben. Für diese Faktoren werden z.B. eigenwertbasierte „Ladungen“ ausgewiesen, welche die Korrelation zwischen einer abhängigen Variable und den zu Faktoren verdichteten erklärenden Variablen angeben. Einen anwendungsorientierten Überblick über dimensionsreduzierende Verfahren geben Backhaus, Erichson, Plinke und Weiber (2005). Faktorenanalytische Verfahren unterstellen, dass ein gemeinsamer, unbeobachteter Faktor für die Ausprägung mehrerer beobachteter Variablen verantwortlich ist (beispielsweise „familiäres Umfeld" für Alkoholismus oder die Wahrscheinlichkeit, eine Dissertation zu schreiben, etc.).

Der Gedanke, anhand dimensionsreduzierender Verfahren einen MetaDatensatz mit Informationen über angewandte Gleichgewichtsmodelle auf wenige, statistisch voneinander möglichst unabhängige und mit der abhängigen Variable stark korrelierte Faktoren zu reduzieren, erscheint zunächst viel versprechend. Allen faktoranalytischen Verfahren ist jedoch gemein, dass sie eine Interpretation der ermittelten Faktoren notwendig machen. Dies bedeutet, dass sich am Beispiel eines angewandten Gleichgewichtsmodells eventuell Faktoren nicht mehr eindeutig auf die ihnen innerhalb eines Modells zu Grunde liegenden Funktionen zurückführen lassen: Hohe Armington-Elastizitäten sind möglicherweise ceteris paribus positiv mit simulierten Wohlfahrtsänderungen in Region $r$ (für $r=1,2, \ldots R$ geografische Regionen in einem Simulationsexperiment) korreliert; die Modellierung steigender Skalenerträge innerhalb einiger produzierender Sektoren würde diese positive Korrelation womöglich ebenfalls aufweisen. Nach Reduzierung auf einen gemeinsamen Faktor, den man beispielsweise als „wohlfahrtssteigernde Einflüsse“ bezeichnen könnte, hätte man lediglich zwei eindeutig identifizierte Modellspezifikationen durch eine neue, möglicherweise intransparentere Kategorie ersetzt und möglicherweise auch Interaktionseffekte solcher Modellspezifikationen ignoriert.

Im Falle angewandter Gleichgewichtsmodelle können theoretisch alle Modellspezifikationen eindeutig beobachtet werden. Die Suche nach einem gemeinsamen, zu Grunde liegenden und unbeobachteten Faktor erübrigt sich also. Vielmehr würde die Zusammenfassung statistisch ähnlich wirkender, aber theoretisch voneinander verschiedener Modellkomponenten eine zusätzliche $\mathrm{Be}$ trachtungsebene einführen, welche ein Verständnis der Simulationsergebnisse in ihrem modelltechnischen Kontext noch um die Ebene der jeweils identifizierten „Faktoren“ ergänzt und wahrscheinlich verkompliziert. Da ein wichtiges Ziel des Projektes in der Verbesserung der Transparenz angewandter Gleichgewichtsmodelle besteht, erscheint eine Anwendung faktoranalytischer Methoden folglich nicht sinnvoll, da ein intersubjektiv wenig reproduzierbarer methodischer Schritt in die Meta-Analyse eingeführt würde. 
Ein ähnliches Verfahren zur Identifizierung gemeinsamer Strukturen innerhalb der Daten ist die Clusteranalyse. Clusteranalyse ist ähnlich wie Faktorenanalyse nur ein Oberbegriff für eine Vielzahl strukturerhellender Verfahren (Backhaus et al. 2005). Typisch für diese Verfahren ist die Anordnung der zu untersuchenden Daten innerhalb eines Koordinatensystems und nachfolgend die anhand eines Distanzmaßes erfolgende Ermittlung der relativen Abstände der Daten zueinander. Die Ergebnisse von Clusteranalysen werden häufig in Form von Dendrogrammen dargestellt. Bei diesen Verfahren bleiben die ursprünglichen Variablen erhalten und gemeinsame Strukturen (Kovarianzen) können erkannt werden.

Der Nachteil clusteranalytischer Verfahren hinsichtlich einer Verwendung dieser Verfahren in einer Meta-Analyse angewandter Gleichgewichtsmodelle besteht darin, dass Clusteranalysen zwar gemeinsame Strukturen innerhalb der Daten aufdecken können, aber nicht die Kausalität zwischen diesen Strukturen und einer abhängigen Variable beschreiben, geschweige denn quantifizieren können. Nichtsdestotrotz können Clusteranalysen eventuell helfen, neue Hypothesen hinsichtlich der Zugehörigkeit unterschiedlicher Modellannahmen zu einem gemeinsamen Modellansatz zu entwickeln. Beispielsweise könnten Annahmen über eine besonders große intersektorale und interregionale Faktormobilität innerhalb von allgemeinen Gleichgewichtsmodellen durch eine Clusteranalyse in unmittelbare Nachbarschaft von partiellen Gleichgewichtsmodellen mit der Spezifikation von besonders großen (long run) Angebotselastizitäten gerückt werden, woran deutlich würde, dass diese beiden grundsätzlich verschiedenen Modellannahmen beispielsweise mit simulierten Wohlfahrtsänderungen c.p. in ähnlicher Weise korreliert sind.

Für eine explorative Analyse eines Meta-Datensatzes kommen ferner sogenannte „Regressionsbäume“ (d.h. die abhängige Variable ist stochastisch verteilt) beziehungsweise „Klassifikationsbäume“ (d.h. die abhängige Variable ist diskret verteilt) in Frage (Breiman, Friedman, Olshen und Stone 1984). Diese nicht parametrische statistische Schätzmethode kombiniert die Stärken der kausalanalytischen Regressionsanalyse und der strukturerhellenden Cluster-Verfahren (Maindonald und Braun 2003, Kutner et al. 2005). Das Grundprinzip dieser Verfahren sieht eine Aufteilung des Datensatzes anhand von Aufteilungskriterien vor, welche so auf den Datensatz angewendet werden, dass ein bestimmtes Fehlermaß wie etwa die Summe der quadrierten Fehlerterme (SSE) minimiert wird. Maindonald und Braun (2003) erläutern eingehend praktische Aspekte bei der Erstellung von Regressions- und Klassifikationsbäumen und diskutieren deren Vor- und Nachteile im Vergleich zu linearen Regressionsmodellen:

Regressionsbäume können mit fehlenden Werten innerhalb der erklärenden Variablen umgehen, ohne entsprechende Observationen vollständig ignorieren 
zu müssen. Interaktionsterme sind in Regressionsbäumen automatisch berücksichtigt und die Interpretation der entstehenden Baumdiagramme ist meist besonders anschaulich. Im Gegenzug sind Regressionsbäume gezwungen, kontinuierliche, erklärende Variablen als Kategorien zu werten; Regressionsbäume erreichen eventuell keinen maximalen Erklärungsgehalt über einen Datensatz, sondern nur lokale Optima; kausale Wirkungszusammenhänge zwischen einer abhängigen und einer erklärenden Variable werden evtl. verschleiert und sehr komplexe Regressionsbäume können unübersichtlich und schwer zu interpretieren sein (Maindonald und Braun 2003, S. 261).

Im Falle angewandter Gleichgewichtsmodelle können Regressions- und Klassifikationsbäume insbesondere hilfreich sein, um spezifische Interaktionseffekte $\mathrm{zu}$ identifizieren, welche nur innerhalb eines bestimmten Modellkontextes einen wichtigen Einfluss auf die abhängige Variable entfalten und im Durchschnitt vieler Studien sowie im Kontext weiterer Modellspezifikation nicht signifikant sind. Regressionsbäume stellen im Falle einer Meta-Analyse angewandter Gleichgewichtsmodelle daher eventuell eine anschauliche, strukturerhellende und kausalanalytische, nicht parametrische Alternative $\mathrm{zu}$ einem klassischen Meta-Regressionsmodell dar.

Insgesamt scheinen die etablierten statistischen Methoden zur Dimensionsreduktion innerhalb großer Datensätze von begrenzter Zweckmäßigkeit hinsichtlich einer Verwendung in der Meta-Analyse. Eventuell können jedoch strukturerhellende Verfahren, insbesondere Regressions- und Klassifikationsbäume, zur Hypothesengenerierung verwendet werden. Zur Analyse und Quantifizierung der Kausalität zwischen simulierten Änderungen bestimmter endogener Variablen innerhalb angewandter Gleichgewichtsmodelle und den sie determinierenden Modellspezifikationen ist jedoch die Schätzung eines parametrischen Meta-Regressionsmodells notwendig.

\subsubsection{Heteroskedastizität}

Eine wichtige Einnahme des Standard-Regressionsmodells besteht in der homogenen Varianz der Fehlerterme $\epsilon$. Im Falle von Heteroskedastizität haben die Fehlerterme jedoch unterschiedliche Varianzen.

Gründe für Heteroskedastizität im Zusammenhang mit einer Meta-Analyse können sehr unterschiedlich sein. Stanley und Jarrell (1989) erwarten Heteroskedastizität aufgrund der Tatsache, dass die Beobachtungen innerhalb eines Meta-Datensatzes aus unterschiedlichen Studien stammen. Im Hinblick auf eine Meta-Analyse angewandter Gleichgewichtsmodelle lassen sich einige weitere Gründe identifizieren, die unter Umständen zu dem Phänomen von Heteroskedastizität führen können: 
Werden in einem Modellkonzept für unterschiedliche Simulationsexperimente beispielsweise verschiedene Datengrundlagen verwendet, so könnten simulierte Wohlfahrtseffekte je nach Datenquelle (Basisjahr, etc.) eine andere Varianz aufweisen. Ferner könnten simulierte Politikänderungen mit vergleichsweise großem Liberalisierungsschritt aufgrund von Interaktionseffekten auch eine größere Variabilität aufweisen als simulierte Politikänderungen mit kleinerem simulierten Liberalisierungsschritt. Im Zeitverlauf könnten angewandte Gleichgewichtsmodelle einem technischen Fortschritt unterliegen, welcher dazu fuhrt, dass über Modelle hinweg die Varianz innerhalb der simulierten Ergebnisse abnimmt. Für jüngere Publikationen würde sich demzufolge eine Konvergenz simulierter Ergebnisse für ein bestimmtes Politik-szenario zu einem bestimmten Mittelwert ausmachen lassen. Einige Autoren (vgl. Bouët 2006) wollen eine solche Konvergenz identifiziert haben und führen dies auf die gestiegene Verbreitung des GTAP-Modells zurück. Zunehmende Dominanz eines bestimmten Modellierungskonzeptes gegenüber vielen isolierten Einzellösungen könnte durchaus ein Indiz für heteroskedastische Meta-Daten sein.

Ein auf den ersten Blick nicht immer leicht von Heteroskedastizität zu trennendes Phänomen sind sog. „Ausreißer“ innerhalb der Daten (Gujarati 2003). Sofern einzelne Simulationsexperimente extrem weit von den meisten übrigen Beobachtungen entfernt liegende Simulationsergebnisse produzieren, kann dies ein Hinweis auf Heteroskedastizität sein, welche durch eine bestimmte, möglicherweise sehr spezielle Modellspezifikation zu Stande kommt (dies könnte beispielsweise eine ansonsten in keinem Modell vorhandene Spezifizierung der Macro-Closure, der Ausgabenfunktion oder einer Produktionsfunktion sein). Sollte das Vorliegen von Ausreißern innerhalb der Daten darauf zurückzufuhren sein, dass diese „Ausreißer“ einem vollkommen anderen Typ von Modellen zuzuordnen sind, so gehören diese in Wirklichkeit einer anderen als der durch die übrigen Beobachtungen abgebildeten Grundgesamtheit an (Gujarati 2003) und es muss sorgfältig fur die relevanten Unterschiede zwischen diesen Grundgesamtheiten kontrolliert werden, da sonst als vermeintliche Heteroskedastizität erscheint, was in Wirklichkeit auf ein Problem fehlender erklärender Variablen zurückzufuihren ist (Rawlings, Pantula und Dickey 1998; Gujarati 2003).

Eine eingehende Darstellung statistischer Tests und Identifikationsverfahren für Ausreißer und Beobachtungen, welche einen besonders starken Einfluss auf eine Regression ausüben, geben Kutner et al. (2005). Eines der am häufigsten in diesem Zusammenhang verwendeten Maße im Rahmen der Validierung eines Regressionsmodells ist Cook's Distance $\mathrm{D}_{i}$ (mit $i=1,2, \ldots N ; N=$ Zahl der Observationen in einem Datensatz): 


$$
D_{i}=\frac{\sum_{j=1}^{n}\left(\hat{Y}_{j}-\hat{Y}_{j(i)}\right)^{2}}{p M S E}
$$

Dabei bezeichnet $\mathrm{D}_{\mathrm{i}}=$ Cook's Distance für Observation $i$, pMSE $=$ Mean Squared Error für alle 1,2,..P; $P=$ Zahl der Regressionskoeffizienten. $\hat{Y}_{j}=$ angepasster Wert für alle Observationen $n$ und $\hat{Y}_{j(i)}=$ angepasster Wert für alle Observationen ohne Observation i. Durch Cook's Distance kann somit der Einfluss einer einzelnen Observation auf die gesamte Regression bestimmt werden (Kutner et al. 1995).

Weitere Gründe fưr Heteroskedastizität können in einer ungleichmäßigen Verteilung bestimmter erklärender Variablen oder in fehlerhaften Formen der Datentransformationen liegen (Rawlings, Pantula und Dickey 1998; Gujarati 2003; Kutner et al. 2005).

Hinsichtlich der ökonometrischen Schätzung eines linearen Regressionsmodells für eine Meta-Analyse angewandter Gleichgewichtsmodelle kann vermutet werden, dass die Meta-Daten Heteroskedastizität aufweisen werden. Die Hypothese der Homoskedastizität ist also anhand geeigneter Tests zu prüfen. In Frage kommen u.a. der verbreitete Breusch-Pagan-Godfrey Test sowie der White Test; der Goldfeld-Quant Test eignet sich aufgrund seiner Begrenzung auf nur eine erklärende Variable wahrscheinlich weniger (Kutner et al. 2005). Es existiert darüber hinaus eine Reihe weiterer statistischer Tests, welche je nach Art der vorliegenden Heteroskedastizität geeignet sind, deren Präsenz innerhalb der Daten zu testen. Funktionsweise und Interpretation dieser Tests unterscheiden sich z.T. deutlich voneinander; eine allgemeine Darstellung erscheint hier dennoch nicht notwendig; vielmehr sei auf entsprechende Standardwerke verwiesen (Greene 2003, Wooldridge 2002).

Unter Heteroskedastizität sind die OLS-Schätzer weiterhin unverzerrt, aber nicht BLUE. Um einen effizienteren Schätzer zu erreichen, muss versucht werden, die Heteroskedastizität zu korrigieren. Hierfür gibt es verschiedene Standardverfahren: Sofern die Varianz der heteroskedastischen Fehlerterme bekannt ist, können verschiedene Gewichtungsschemata auf die abhängige Variable angewendet werden, was bedeutet, dass statt des OLS-Schätzers ein WLS bzw. GLS (weighted least square bzw. generalized least square) Schätzer verwendet wird (Rawlings, Pantula und Dickey 1998, Greene 2003).

Ferner kann versucht werden, die Struktur der Heteroskedastizität innerhalb der Daten abzuschätzen und nicht die Fehlerterme durch Gewichtung zu normieren, sondern die abhängigen und/oder erklärenden Ausgangsbeobachtungen so zu transformieren, dass Heteroskedastizität auf diese Art beseitigt wird (Rawlings, Pantula und Dickey 1998). Im Falle eines größeren Samples, 
was bei einer Meta-Analyse angewandter Gleichgewichtsmodelle wahrscheinlich zu erwarten ist, kann Heteroskedastizität auch bei unbekannter Fehlervarianz begegnet werden, indem sog. „robuste“ (oder auch „Huber-White“-, „White“-, etc.) Standardfehler verwendet werden. Diese sog. robusten Schätzverfahren basieren auf einer geschätzten Normierung der Fehlertermvarianz und können auch erweitert werden, um Cluster-Strukturen innerhalb der Daten zu berücksichtigen (vgl. z.B. Wooldridge 2002, Kutner et al. 1995, S. 437ff.). Im Hinblick auf eine Meta-Analyse angewandter Gleichgewichtsmodelle stellen diese robusten Schätzverfahren eine verbreitete Möglichkeit dar, BLUE-Schätzungen zu erzielen; im Falle von Autokorrelation zusätzlich zur Heteroskedastizität können evtl. sog. "Newey-West" Standardfehler eingesetzt werden (Greene 2003, Wooldridge 2002).

\subsubsection{Serielle Korrelation/Autokorrelation}

Autokorrelation bedeutet allgemein, dass die Fehlerterme $e$ bzw. $\mathrm{u}_{\mathrm{i}}$ nicht unabhängig voneinander sind, d.h. die folgende Annahme ist verletzt:

$$
\operatorname{Cov}\left(u_{m}, u_{n}\right)=0, \forall m \neq n
$$

Dies wird meist auf Zeitreihen bezogen, da diese Verletzung einer Grundannahme des Standard-Regressionsmodells hier besonders häufig beobachtet werden kann. Hinsichtlich einer Meta-Analyse angewandter Gleichgewichtsmodelle müssen grundsätzlich mehrere Zeitachsen und damit mehrere mögliche Ursachen für Autokorrelation unterschieden werden:

Die Meta-Analyse betrachtet zunächst ein Literatursample mit Publikationen aus verschiedenen Publikationsjahren. Innerhalb dieser Publikationen werden Modelle verwendet, welche auf Basisdaten zurückgreifen. Diese Basisdaten sind meist einige Jahre älter als das jeweilige Publikationsjahr. Die weithin verwendeten GTAP-Datenbanken erscheinen ca. alle drei Jahre, aber die darin verwendeten Basisdaten sind jeweils ca. 5 Jahre älter als das entsprechende Erscheinungsjahr der Datenbank. Typische Zeitreihenphänomene wie beispielsweise eine systematische Korrelation von $u_{t}$ mit $u_{t-1}$ erscheinen im Zusammenhang mit den Basisdatenjahren zunächst nicht als vordringliches Problem, da keine GTAP Datenbasis von ihrer unmittelbaren Vorgängerin stärker beeinflusst sein sollte als von allen anderen Vorgänger-GTAP-Datenbanken.

Das Phänomen der Autokorrelation kommt jedoch evtl. auf der Zeitachse des Publikationsjahres zum Tragen, wenn man annimmt, dass nachfolgende Autoren sich an den Ergebnissen ihrer Vorgänger orientieren, d.h. die erste Publikation zu einem Politikszenario (z.B. Agenda 2000 der Common Agricultural Policy CAP) etabliert einen Ausgangswert, an welchem sich nachfolgende 
Publikationen orientieren. Es gibt allerdings keinen eindeutigen Hinweis darauf, ob eine solche Orientierung nachfolgender Autoren an vorangegangenen Studien generell einen fallenden oder einen steigenden Trend der Simulationsergebnisse (etwa durch die subjektive Auswahl bestimmter Modellspezifikationen durch die Modellierer) induzieren wird, sodass eine stochastische Verteilung dieses Effektes angenommen werden kann. Rawlings, Pantula und Dickey (1998) empfehlen, zunächst jeden Datensatz, der wiederholte Messungen aufweist, als Zeitreihe zu betrachten; Autokorrelation wird allgemein jedoch wahrscheinlich von untergeordneter Bedeutung innerhalb des Meta-Datensatzes sein, was aber ebenfalls statistisch überprüft werden muss (z.B. Durbin-Watson Test, vgl. Rawlings, Pantula und Dickey 1998). Man unterscheidet darüber hinaus verschiedene Arten von serieller Fehlerterm-Korrelationen, welche hier nicht näher ausgeführt werden sollen (vgl. dazu Greene 2003, Kutner et al. 2005).

\subsubsection{Fixed-, Random- oder Mixed-Effects}

Die im Fall von serieller Korrelation verletzte Unabhängigkeit der Fehler $e$ untereinander kann auch aus anderen als den in Kapitel 3.2.5 genannten Gründen innerhalb eines Datensatzes vorliegen: $\mathrm{Da}$ jede Studie innerhalb des MetaDatensatzes auf ein bestimmtes Modellkonzept zurückgreift, welches normalerweise bestimmte studienspezifische Modifikationen (Closure, Datenbankprojektion, Datenaggregation, etc.) aufweist und mit welchem ein oder mehrere Simulationsexperimente (z.B. alternative Zollkürzungsformeln) durchgeführt werden, sind einzelne Observationen (z.B. simulierte regionale Wohlfahrtsveränderungen) möglicherweise miteinander korreliert.

$\mathrm{Da}$ jede Studie und jedes Simulationsexperiment durch eine individuelle $\mathrm{Zu}$ sammenstellung des experimentellen Aufbaus gekennzeichnet sind, welcher normalerweise nicht über Studien hinweg wiederholt wird, spricht man bei dieser Abhängigkeitsstruktur der Fehlerterme von hierarchischen oder genesteten Strukturen. Dabei wird eine solche Datenstruktur insbesondere im Bereich psychologischer und soziologischer Studien als hierarchisch bezeichnet (Goldstein 1995); beispielsweise im Bereich der Biometrie wird hingegen von genesteten Daten gesprochen (Kutner et al. 2005). Eine ökonometrische Modellierung solcher Strukturen ist in Meta-Analysen häufig notwendig (Bateman und Jones 2003). Dabei kommen meist lineare Modelle zum Einsatz. Je nach Struktur der Fehlertermabhängigkeit werden zusätzlich zu dem stets auch im linearen Standardregressionsmodell (Gleichung 3.2 a) vorhandenen Random Effect $\epsilon$ mitunter auch weitere Random Effects eingeführt. Ein solches lineares Regressionsmodell, welches neben Fixed Effects mehrere Random Effects enthält, wird innerhalb der Literatur meist als Linear Mixed Model bezeichnet (auch die Bezeichnungen hierarchische-, oder multilevel Modelle sind gebräuchlich). 
Dabei ist zu beachten, dass unter Fixed Effects im Zusammenhang mit Linear Mixed Models alle Kovariate bezeichnet werden, für die Koeffizienten über alle hierarchischen Strukturen (d.h. Datengruppen) hinweg geschätzt und interpretiert werden - unabhängig davon, wie diese Variablen verteilt sind, was einen Unterschied zur Terminologie im Bereich von Panel-Modellen darstellt (vgl. Goldstein 1995, Pinheiro und Bates 2000 einerseits und Wooldridge 2002, Hsiao 2003 andererseits). Generell unterscheidet man Random Intercept und Random Coefficients, d.h. der Random Effect kann sowohl als gruppenspezifischer Achsenabschnitt als auch als gruppenspezifische Regressionsgerade spezifiziert werden. In diesem Fall werden innerhalb der spezifizierten Random Effects ebenfalls Kovariate eingesetzt, aber die hierfür geschätzten Koeffizienten sind datengruppenspezifisch und daher Teil einer (normal)Verteilung, d.h diese Random Effects Coefficients werden wie Fehlerterme behandelt. Gleichung (3.9) verdeutlicht diesen Zusammenhang für das Beispiel einer ökonometrischen Meta-Analyse angewandter Gleichgewichtsmodelle. Dabei wird das lineare Meta-Modell von Stanley und Jarrell (1989, vgl. Gleichung 3.3) durch Einführung eines Random Effects zu einem linearen Mixed Effects Meta-Modell erweitert.

Um die Zahl der Datengruppen dabei übersichtlich zu gestalten, wird nachfolgend zunächst unterstellt, dass jede Studie $j(j=1,2, \ldots, L ; L=Z$ Zahl der Studien im Meta-Datensatz) nur ein Simulationsexperiment durchführt, wodurch eine zusätzliche Datengruppierung auf Ebene der Simulationsexperimente innerhalb jeder Studie entfällt:

$$
b_{r j}=\beta+\sum_{k=1}^{K} \alpha_{k} Z_{r j k}+\sum_{m=1}^{M} \gamma_{j m} X_{r j m}+e_{r j}
$$

Mit: $b_{\mathrm{rj}}$ bzw. nachfolgend $Y_{\mathrm{rj}}=$ simulierter Wohlfahrtsänderungen auf regionaler Ebene $r$ in Studie $j ; r=1,2, \ldots, R$ für $R$ aggregierte Regionen innerhalb des Simulationsexperiments in Studie $j(j=1,2, \ldots, L$ für $L$ Studien); da es sich im Falle angewandter Gleichgewichtsmodelle bei $b_{i j}$ nicht um geschätzte Koeffizienten innerhalb eines Meta-Datensatzes wie in Gleichung (3.3) handelt, wird $b_{\mathrm{rj}}$ nachfolgend durch die Bezeichnung $Y_{\mathrm{r}}$ ersetzt;

$Z_{r j}=$ erklärende Meta-Variable $(\mathrm{k}=1,2, \ldots, K$ für $K$ erklärende Meta-Variablen);

$\alpha=$ zu schätzende Koeffizienten für $k=1,2, \ldots, K$ erklärende Meta-Variablen über Studien und Regionen hinweg (gemäß der Mixed Model Terminologie sind dies die Fixed Effects);

$\gamma_{j m}=$ Random Coefficients auf Ebene von Studie $j$ für $m=1,2, \ldots, M$ erklärende Variablen,

$X_{\text {rjm }}=$ Random Coefficients Kovariate, für $m=1,2, \ldots, M$ erklärende Variablen.

$E_{r j}=$ Fehler,

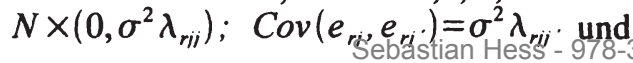




$$
\gamma_{j m} \wedge N\left(0, \Psi_{m}^{2}\right), \operatorname{Cov}\left(\gamma_{m}, \gamma_{m}\right)=\Psi_{m m} .
$$

Sofern für die $b_{r}$ Observationen gilt, dass sie innerhalb jeder Studie $j$ stochastisch unabhängig voneinander sind und konstante Fehlertermvarianzen aufweisen, ist

$$
\lambda_{r i j}=\sigma^{2} \text { und } \lambda_{r i j}=0 \text { für } j \neq j^{\prime}
$$

Aufbau und Funktionsweise eines solchen Linearen Mixed Effects Modells im Rahmen einer Meta-Analyse angewandter Gleichgewichtsmodelle soll anhand des folgenden Beispiels nachvollzogen werden und ist an Beispiele für einfache Mixed Effects Modelle aus Pinheiro und Bates (2000) sowie Fox (2002) angelehnt. Soweit nicht anders erwähnt, finden dabei die gleichen im Zusammenhang mit Gleichung (3.9) erläuterten Variablen und Koeffizienten Verwendung:

$$
Y_{r j}=\alpha_{0 \mathrm{j}}+\alpha_{1 \mathrm{j}} \Delta P_{r j}+e_{r j}
$$

Gleichung (3.10 a) erklärt simulierten regionalen Wohlfahrtsgewinn $Y_{r j}$ dabei zunächst als Funktion einer simulierten Politikänderung $\Delta P_{r j}$, beispielsweise einer Kürzung des MFN Importzolls in Region $r$. Dabei wird unterstellt, dass sowohl die durchschnittliche Wohlfahrtsänderung je Studie $\left(\alpha_{0 j}\right)$ als auch die marginale Änderung der regionalen Wohlfahrt als Folge von $\Delta P$ in Region $r$ und Studie $j$ eine Funktion des Basisjahres der verwendeten Basisdaten sowie des allgemeinen Modellkonzepts (GTAP, ATPSM, etc.) ist:

$$
\begin{aligned}
& \alpha_{0 j}=\alpha_{00}+\alpha_{01} \text { Datenjahr }_{j}+\alpha_{02} \text { Modellkonzept }_{j}+\gamma_{0 j} \\
& \alpha_{1 j}=\alpha_{10}+\alpha_{11} \text { Datenjahr }_{j}+\alpha_{12} \text { Modellkonzept }_{j}+\gamma_{1 j}
\end{aligned}
$$

Dabei stellen $\gamma_{0 j}$ und $\gamma_{l j}$ zusätzlich zum Fehlerterm $e_{r j}$ eingeführte Random Effects auf Ebene der Studie j dar (gemäß Annahme ist dies auch die Ebene des jeweils einzigen Simulationsexperiments in Studie j). Es wird somit unterstellt, dass innerhalb jeder Studie eigene Fehlerterme existieren, welche bei Nichtbeachtung Teil des Fehlerterms $e_{r j}$ wären und dessen angenommene Unabhängigkeit evtl. verletzen würden.

Durch einfaches Einsetzen der Zusammenhänge in den Gleichungen (3.10 b) in die Gleichung ( 3.10 a) ergibt sich:

$$
\begin{gathered}
Y_{r j}=\alpha_{00}+\alpha_{01} \text { Datenjahr }_{j}+\alpha_{02} \text { Modellkonzept }_{j}+\gamma_{0 j} \\
+\left(\alpha_{01}+\alpha_{11} \text { Datenjahr }_{j}+\alpha_{12} \text { Modellkonzept }_{j}+\gamma_{1 \mathrm{j}}\right) \Delta P_{r j}+e_{r j}
\end{gathered}
$$


In Anlehnung an Gleichung (3.9) kann diese Gleichung (3.11 a) umgeformt werden, wobei $\alpha_{00}$ die durchschnittliche Wohlfahrtsveränderung im Mittel des Meta-Datensatzes über Studien hinweg ausweist:

$$
\begin{gathered}
Y_{r j}=\beta_{0}+\alpha_{1} \text { Datenjahr }_{j}+\alpha_{2} \text { Modellkonzept }_{j}+\alpha_{3} \Delta P_{r j}+\alpha_{4} \text { Datenjahr }_{j} \Delta P_{r j} \\
+\alpha_{5} \text { Modellkonzept }_{j} \Delta P_{r j}+\gamma_{0 j}+\gamma_{1 j} \Delta P_{r j}+e_{r j}
\end{gathered}
$$

Die Terme $\gamma_{0 j}$ und $\gamma_{l j} \Delta P_{r j}$ stellen dabei Random Intercept und Random Coefficient für $\Delta P_{r j}$ dar, d.h. es wird unterstellt, dass innerhalb jeder Studie die simulierte Politikänderung $\Delta P_{r j}$ eine eigene Regressionsgerade aufweist, deren Steigung und Achsenabschnitt über Studien hinweg verschieden und Teil einer Zufallsverteilung möglicher Wirkungen simulierter Politikänderungen innerhalb von Gleichgewichtsmodellen ist. Die Signifikanz eines solchen Random Effects kann durch Auslassen innerhalb des Mixed Effects Modells und Vergleich der alternativen Modelle mit und ohne Random Effect anhand ihrer AIC/BIC Werte getestet werden; sofern ein Mixed Effects Modell keinen signifikant besseren AIC/BIC Wert aufweist als das entsprechende Modell ohne einen bestimmten Random Effect, wird dieser als nicht signifikant angesehen (Pinheiro und Bates 2000, für die Signifikanz eines Random Effects in Panel Modellen vgl. Hausman Test, Wooldridge 2002). Die Signifikanz eines solchen Random Effects innerhalb eines Meta-Modells angewandter Gleichgewichtsmodelle würde daher möglicherweise darauf hindeuten, dass wichtige erklärende Variablen bzw. wichtige Interaktionseffekte erklärender Variablen innerhalb des Modells (z.B. im Zusammenhang mit der Messung von $\left.\Delta P_{r j}\right)$ nicht erfasst werden konnten.

An diesem Beispiel wird deutlich, dass die Einfuhrung eines Random Effects im Zusammenhang mit einer Meta-Analyse angewandter Gleichgewichtsmodelle wahrscheinlich nur begrenzt zur Formulierung eines transparenzverbessernden ökonometrischen Meta-Modells beitragen wird: Da es sich um deterministische Modelle handelt, deren einzelne Komponenten bekannt sein sollten und individuell benannt werden können, ist zunächst nicht einleuchtend, warum beispielsweise die simulierte Politikänderung $\Delta P_{r}$ oder eine anderweitige Komponente des experimentellen Aufbaus je nach Studie $j$ (d.h. datengruppenspezifisch) einen eigenen Achsenabschnitt und eigene Steigung aufweisen sollte, ohne dass modellimmanente Einflussgrößen identifizierbar sein wären, welche für diese unterschiedlichen datengruppeninternen Effekte von $\Delta P_{r j}$ verantwortlich sind und innerhalb der Regressionsgleichung ebenfalls als erklärende Variablen aufgenommen werden könnten.

Lineare Mixed Effects Modelle werden üblicherweise mit dem Restricted Maximum Likelihood Schätzer (REML) geschätzt, was einer um Freiheitsgrade 
korrigierten Schätzung mit Maximum Likelihood Schätzer gleichkommt (Pinheiro und Bates 2000, Fox 2002).

\subsubsection{Messfehler}

Messfehler können sowohl in der abhängigen als auch in den erklärenden Variablen auftreten. Ein (systematisch) Messfehler in der abhängigen Variable ist Teil des Fehlerterms und stellt daher meist keine grundsätzlichen Schwierigkeiten für eine Regression dar. Dies gilt nicht für Messfehler in den erklärenden Variablen. Ein solcher Messfehler $\tau$ in einer erklärenden Variablen wird allgemein definiert als:

$$
\tau_{i}=X_{i}^{*}-X_{i}
$$

wobei $X_{i}$ die wahre und $X_{i}^{*}$ die unvollkommen gemessene Variable für Observation i, $i=1,2, \ldots, N ; N=$ Zahl der Beobachtungen im (Meta-)Datensatz darstellt. Durch Einsetzen in die allgemeine Meta-Regression (Gleichung 3.3) ergibt sich entsprechend für $\tau_{i}=Z_{i}^{*}-Z_{i}$, sofern $Z$ eine Variable der erklärenden Vektoren $M, P$ und $D$ aus Gleichung (3.4) ist:

$$
Y_{i}=\beta_{0}+\beta_{1} Z_{i}^{*}+\left(e_{i}-\alpha_{i} T_{i}\right) \quad i=1,2, \ldots, N .
$$

Für die Schätzung des Koeffizienten $\alpha$ ergibt sich, dass $\alpha_{i}^{*} \leq \alpha$, d.h. der geschätzte Koeffizient wird durch den Messfehler in $Z_{i}$ gedämpft sein („Attenuation Bias"). Das Ausmaß dieser Dämpfung hängt von der relativen Größe der Varianz der abhängigen Variable $\left(\sigma_{y}^{2}\right)$ zu der erklärenden Variable ( $\sigma_{Z}^{2}$ ) ab: Ist $\sigma_{Y}^{2}$ relativ zu $\sigma_{Z}^{2}$ klein, wird auch die Dämpfung gering ausfallen (Kutner et al. 2005).

Dem Dämpfungsbias kann evtl. begegnet werden, indem die Varianzen $\sigma_{Y}^{2}$ und $\sigma_{z}^{2}$ geschätzt werden und so Erkenntnisse über seine Höhe gewonnen werden können, oder indem Proxyvariablen für $X_{i}$ (bzw. $Z_{i}$ ) gefunden werden, welche nicht mit $\tau_{\mathrm{i}}$ korreliert sind (Kutner et al. 2005, Wooldridge 2002).

Übertragen auf eine Meta-Analyse angewandter Gleichgewichtsmodelle bedeutet dies, dass Messfehler in den erklärenden Variablen evtl. leicht vermieden werden können, indem die kausale Struktur innerhalb eines Gleichgewichtsmodells möglichst genau eruiert wird. Dies ermöglicht es, Variablen zu identifizieren, welche fehlerfrei messbar sind und weniger gut quantifizierbare Effekte mit einschließen oder approximieren. 


\subsubsection{Die Annahme der Linearität}

Angewandte Gleichgewichtsmodelle bestehen meist aus nicht-linearen Gleichungssystemen. Arndt (1996) argumentiert beispielsweise, dass das Verhalten des GTAP-Modells durch ein Polynom dritten Grades approximiert werden kann. Ein wichtiger Zusammenhang zwischen allokativen Wohlfahrtseffekten und Zöllen besagt, dass die Höhe des Wohlfahrtsverlustes in einem Sektor eine quadratische Funktion des Zolles ist (Nicholson 1998). Es ist daher zunächst nicht einleuchtend, weshalb ein lineares Regressionsmodell eine sinnvolle Abbildung simulierter Wohlfahrtseffekte ermöglichen könnte.

Andererseits ist die Verwendung linearer Regressionsmodelle meist der Ausgangspunkt für Meta-Modellierung im Bereich anderer wissenschaftlicher Simulationsmodelle (Kleijnen et al. 2005), die ebenfalls häufig durch nichtlineare Zusammenhänge gekennzeichnet sind (Banks 1998). Kleijnen et al. (2005) empfehlen den Einsatz nicht-parametrischer Schätzer zur Identifizierung funktionaler Zusammenhänge innerhalb von Meta-Modellen, wobei die Autoren darauf verweisen, dass meist ein parametrisches Regressionsmodell linearer oder quadratischer Ordnung am Ende eines Meta-Modellierungsprozesses stehen sollte, um die Verhaltensweise des eigentlichen Modells approximativ und in leicht zugänglicher Form darstellen zu können. Mathematisch handelt es sich also bei einem linearen Meta-Modell angewandter Gleichgewichtsmodelle um eine Taylor-Approximation erster Ordnung. Hierbei ist nicht die Frage nach dem generellen Funktionsverlauf innerhalb des Simulationsmodells entscheidend, sondern nur die Frage, ob diese lineare Approximation den durch sie abgebildeten Bereich des Simulationsmodells hinreichend genau approximiert. Kleijnen et al. (2005) verweisen darauf, dass dies selbst bei insgesamt hochgradig nicht-linearen Simulationsmodellen in entsprechend ausgewiesenen Bereichen der Modelloberfläche möglich ist.

Im Falle angewandter Gleichgewichtsmodelle kann dies bedeuten, dass evtl. ein Subsample von Modellen und Simulationsexperimenten in hinreichend engen Intervallen wichtiger erklärender Variablen ausgewählt werden muss, um eine lineare bzw. quadratische Spezifikation des „Meta-Modells“ zu gewährleisten.

\subsubsection{Modellselektion und Vorhersage}

Aus den Betrachtungen in Kapitel 3.1 ergibt sich die Frage, wie die „Validität“ bzw. „Güte“ eines Meta-Modells determiniert werden kann, und welche Vorhersagen anhand eines Meta-Regressionsmodells über durchschnittlich zu erwartende simulierte Wohlfahrtseffekte allgemein getroffen werden können. Maindonald und Braun (2003) stellen heraus, dass ein "gutes“ statistisches 
Modell insbesondere in der Lage sein muss, die zu Grunde liegenden Beobachtungen zu replizieren und Vorhersagen auf Basis ähnlicher Beobachtungen zu ermöglichen. Je niedriger die Abweichungen der prognostizierten Werte $\left(\hat{Y}_{i}\right)$ von den tatsächlichen Beobachtungen $\left(Y_{i}\right)$, desto besser ist das Modell.

Welche Aussagen können anhand von Meta-Regressionen über durchschnittlich von Simulationsmodellen innerhalb des Literatursamples ausgewiesene Wohlfahrtsveränderungen getroffen werden?

Die predicted values $\left(\hat{Y}_{i}\right)$ eines linearen Regressionsmodells können durch Einsetzen bekannter oder innerhalb des Datenbereichs der bekannten Observationen angesiedelter Beobachtungen in die Regressionsgleichung errechnet werden. Dabei kann das Problem der Hidden Extrapolations auftreten, wobei neu hinzugezogene, erklärende Observationen zunächst dem Anschein nach im Datenbreich des Regressionsmodells liegen, aber in Wirklichkeit eine möglicherweise unzulässige Extrapolation des Regressionsmodells darstellen, da diese Observationen durch den gemeinsam erfassten Bereich der Kovariate nicht abgedeckt werden. Kutner et al. (2005) empfehlen durch Vergleich der leverage Werte $h_{\text {ii }}$ (für $i=1,2, \ldots, N$ Observationen im Datensatz) mit den leverage Werten der neuen Observationen $h_{\text {neu,neu }}$ auf Hidden Extrapolation zu testen. Diese leverage Werte $\mathrm{h}$ sind dabei Elemente auf der Diagonalen der Hat-Matrix (Kutner et al. 2005, S. 400); liegen die Werte für $h_{\text {neu,neu }}$ weit außerhalb des Bereichs der Werte für $h_{\mathrm{i}}$, muss eine möglicher Weise unzulässige Extrapolation über den Bereich des Regressionsmodells hinaus antizipiert werden. Ansonsten können die predicted values $\hat{Y}_{i}$ durch Einsetzen neu hinzugezogener Observationen in die geschätzte Regressionsgleichung errechnet werden.

Die Errechnung von Standardfehlern für diese mittleren errechneten Werte $\hat{Y}_{i}$ der abhängigen Variable (predicted values) ist jedoch eine Funktion der Varianzen der einzelnen Regressionskoeffizienten $b_{k}(k=1,2, \ldots, K$; für $K$ geschätzte Koeffizienten innerhalb der Regression) sowie der Kovarianzen zwischen diesen Koeffizienten $b_{k}$. Der Standardfehler $s$ der predicted values $s\left(\hat{Y}_{\text {neu }}\right)$ für datensatzfremde Oberservationen (siehe Kutner et al. 2005, S. 229) kann jedoch mit den meisten Statistikprogrammen problemlos errechnet werden.

Predictions sind allerdings nur sinnvoll, wenn das zu Grunde liegende Regressionsmodell eine hinreichend genaue Schätzung kausaler Zusammenhänge innerhalb der Daten liefert. Als verbreitetes $\mathrm{Maß}$ wird in diesem $\mathrm{Zu}-$ sammenhang die Summe der quadrierten Fehlerterme (SSE) betrachtet:

$$
S S E=\sum_{i=1}^{n}\left(Y_{i}-\hat{Y}\right)^{2}
$$

Entsprechend ist ist die Summe aller quadrierten Abweichungen zwischen den Beobachtungen $i$ und dem Mittelwert eines Samples: 


$$
S S T=\sum_{i=1}^{n}\left(Y_{i}-\bar{Y}\right)
$$

Ein gebräuchliches, hieraus abgeleitetes $\mathrm{Maß}$ zur Validierung eines ökonometrischen Modells ist der Determinationskoeffizient $R^{2}=1-$ SSE/SST. Da $R^{2}$ bei zusätzlich aufgenommenen erklärenden Variablen nicht kleiner werden kann, empfiehlt sich für Modellvergleiche das angepasst $\mathbf{R}^{2}$ welches die Zahl der Freiheitsgrade mit einbezieht (Kutner et al. 2005).

Goldberger (1998) stellt heraus, dass es im Bereich ökonomischer Fragestellungen nicht notwendigerweise entscheidend sein muss, besonders hohe $\mathrm{R}^{2}$ Werte anzustreben, da im Hinblick auf die Analyse komplexer politischer oder sozialer Phänomene diejenigen Variablen mit dem höchsten Erklärungsgehalt (gemessen in erklärter Varianz in Y) evtl. durch politische Entscheidungen gar nicht beeinflussbar sind. Die ausschließliche Maximierung von $\mathrm{R}^{2}$ kann folglich eventuell an der Identifizierung eines politisch relevanten Modells vorbeifuhren, oder umgekehrt auch trotz niedriger $\mathrm{R}^{2}$ Werte kann ein ökonometrisches Modell relevante Aussagen enthalten (Goldberger 1998). Einen ausführlichen Überblick über weitere Kriterien zur Modellselektion auf Basis des Maßes der quadrierten Fehlerterme (SSE), beispielsweise das ebenfalls weithin verwendete Akaike's Information Criterion (AIC); unter Standardannahmen ist AIC $=n \ln (\mathrm{SSE})-n \ln n$ $+2 k$; ( $n=Z$ Zahl der Observationen, $k=Z$ Zahl der Parameter in einem Regressionsmodell), sowie alternativer Ansätze zur Modellselektion bieten Kutner et al. (2005).

\subsection{Das Literatursample}

Aufgrund der insbesondere im Falle transdisziplinärer Fragestellungen schwer zu überschauenden Fülle wissenschaftlicher Publikationen hat sich die $\mathrm{Zu}$ sammenfassung und Aufbereitung von Forschungsergebnissen als eigene methodische Disziplin etabliert. Diese Disziplin wird sowohl qualitativ im Rahmen von Literaturüberblicken (Surveys) als auch quantitativ im Rahmen von Vote Countings und Meta-Analysen betrieben (Cooper 1998).

Angewandte ökonomische Fragestellungen (Agrarökonomie, Forstökonomie, Umweltökonomie, etc.) sind hierbei keine Ausnahme: vielmehr macht die Vielfalt inhaltlicher Fragestellungen, methodischer Applikationen und transdisziplinärer Kooperationen die Meta-Synthese von Forschungsergebnissen notwendig und stellt gleichzeitig eine besondere methodische Herausforderung dar (Button und Jongma 1995, Stanley 2001, Florax 2002). 
Wesentliches Element dieser methodischen Herausforderung ist der sogenannte Publication Bias (Stanley 2005). Publication Bias bedeutet, dass grundsätzlich jede spezifische Auswahl von Literatur, gleichgültig ob im Rahmen der Bibliographierung eines Forschungsstandes oder als Grundlage für eine MetaAnalyse, von einem möglichen Sample Selection Bias bedroht sein kann. Dieser Bias kann vielfältige Ursachen haben (Stanley 2005). In der Ökonomie ist beispielsweise Publication Bias zu Gunsten signifikanter Ergebnisse gegenüber nicht signifikanten hinlänglich bekannt: Liegt Publication Bias vor, so liefern publizierte Studien zu einer bestimmten Fragestellung häufig kein repräsentatives Abbild der tatsächlich zu dieser Fragestellung existierenden Empirie.

Eine agrarökonomische Fragestellung stellt häufig eine Verbindung aus methodischen und thematischen Aspekten dar. Interdisziplinäre Literaturrecherche zur effektiven Synthese relevanter Literaturbereiche ist damit eine Schlüsselmethode, welche den Erfolg agrarökonomischer Forschung maßgeblich bestimmt, wobei allerdings bisher keine allgemein verbreiteten Recherchemethoden existieren, welche der interdisziplinären Struktur angewandt-ökonomischer Literatur Rechnung tragen. Dieser Abschnitt stellt ein systematisches Verfahren zur Literaturrecherche im Rahmen agrarökonomischer Fragestellungen vor. Das Ergebnis einer solchen Recherche wird nachstehend als „Literatursample“ bezeichnet, da es beansprucht, die relevante Literatur repräsentativ abzubilden, ohne die Such- und Informationsverarbeitungskosten einer Vollerhebung tragen zu müssen. Ein solches „Literatursample“ ermöglicht repräsentative und bei geringem Dokumentationsaufwand intersubjektiv zumindest asymptotisch reproduzierbare Literaturlisten. Das Verfahren wird anhand der Erstellung eines Samples zu simulationsmodell-basierten Analysen im Rahmen der WTO-Agrarverhandlungen während der Doha-Runde illustriert. Das Verfahren wurde als Grundlage für die Meta-Analyse angewandter Gleichgewichtsmodelle im Bereich angewandter, interdisziplinärer Literatur entwickelt, kann aber grundsätzlich als Leitfaden für jede wissenschaftliche Recherche innerhalb der Agrarökonomie dienen, sofern diese den Anspruch erhebt, zu einer bestimmten Fragestellung die relevante Literatur vollständig zu erfassen oder repräsentativ abzubilden.

\subsubsection{Die Literatur-Grundgesamtheit: Simulationen zur Doha-Runde}

Durch die globale Vernetzung von Informationsquellen und die weltweit stark gestiegene Forschungstätigkeit sowie den technischen Fortschritt im Bereich der Textverarbeitung und Kommunikation ist für viele wissenschaftliche Fragestellungen die Menge der potenziell relevanten Literatur aus Sicht eines einzelnen Forschers oft schwierig zu überschauen. Die Ermittlung eines Forschungsstandes zu einer spezifischen Fragestellung erfordert meist die Synthese von 
Erkenntnissen aus mehreren unterschiedlichen Disziplinen, welche jeweils ihre spezifischen Fachtermini entwickelt haben.

Da technischer Fortschritt die Recherche im globalen Literaturbestand ermöglicht, stellt die grundsätzliche Verfügbarkeit von Informationen nur noch selten einen begrenzenden Faktor für Forschung dar; vielmehr steht das Problem der Eingrenzung relevanter Literatur für eine spezifische Fragestellung im Vordergrund (Heckman 2001). Weniger Quantität, sondern vielmehr Qualität und Relevanz der zu berücksichtigenden Literatur stellt also die zentrale Herausforderung für wissenschaftliche Recherche dar. Sofern in die Ermittlung eines Forschungsstandes nicht alle relevanten Informationen eingehen, wird die Generierung neuer Hypothesen fast zwangsläufig die wissenschaftliche Frontier auf ihrem Gebiet verfehlen.

Ferner kommen die erwähnten Kosten der Informationsverarbeitung ins Spiel: wie viel Literatur kann bewältigt werden, d.h. wie präzise muss eine Suche wirken? Für die meisten Literaturrecherchen können in diesem Zusammenhang im Bereich angewandter ökonomischer Forschung folgende Grundsätze angenommen werden:

Die Forschungsfrage besteht aus einer methodischen und aus einer thematischen Komponente, beispielsweise der gleichgewichtsmodell-basierten Modellierung von WTO-Szenarien des Weltagrarhandels.

Es existieren terminologische Begriffe, welche einzeln oder in Kombination die angestrebte Literatur-Grundgesamtheit möglichst eindeutig identifizieren.

Es ist wahrscheinlich, dass unterschiedliche Schulen und Disziplinen Termini entwickelt haben, welche im Sinne der angestrebten Grundgesamtheit synonyme Suchwort-Funktion besitzen.

Die gesamte Ebene relevanter Literatur um eine Fragestellung kann also nur erfasst werden, wenn alle Termini Technici samt ihrer Synonyme sorgfältig ermittelt wurden. Sofern diese Annahmen für die zu recherchierende Literatur zutreffen, kann die Identifizierung eines Literatursamples anhand folgender Arbeitsschritte vorgenommen werden:

1. Genaue inhaltliche Definition der zu erfassenden Literatur.

2. Genaue Definition der zu erfassenden Publikationsformen (z.B. nur PeerReview oder auch Einschluss „grauer“ Literatur?)

3. Auswahl von Datenbanken, welche diesen Bestand repräsentieren.

4. Erstellung einer Suchwortmatrix, welche aus diesen Datenbanken die relevanten Treffer filtert. Diese Tätigkeit erfordert einige Fachkenntnis. Sollte diese nicht vorhanden sein, muss eine explorative Suche bzw. die Konsultation von Experten (,informal colleges“) vorangehen, um die relevanten Fachtermini präzise zu ermitteln.

5. Vollständige Erfassung aller Treffer zu dieser Suchwortmatrix aus allen Datenbanken, Bereinigung um Duplikate. 
6. Stichprobenvergleich mit den Literaturlisten thematisch verwandter Surveys; als Folge evtl. Anpassung der Datenbankauswahl und Suchwortmatrix.

7. Verarbeitung der Literatur innerhalb des Literatursamples durch vollständige Verarbeitung oder Ziehung von Zufallsstichproben.

Vor Beginn der eigentlichen Recherche müssen also relevante Informationsquellen, d.h. Datenbanken (= Bibliothek, Internetportal, Suchmaschine, Fachdatenbank etc.) festgelegt werden (Enneking 1997). Dieser Schritt bildet das Fundament des zu erstellenden Literatursamples: Da jede Datenbank durch ihr Sammelgebiet und ihre Abfragemaske eingegebene Suchanfragen auf eine bestimmte, von anderen Datenbanken verschiedene Weise interpretieren wird, muss jede Datenbank im Sinne eines Literatursamples als eigenes Stratum behandelt werden. Eine Auswahl repräsentativer Strata determiniert also die Repräsentativität des späteren Samples.

Die Menge der für Literaturrecherchen zur Verfügung stehenden Ressourcen (Suchmaschinen, Datenbanken) ist auf den ersten Blick unübersichtlich und verwirrend - u.a. da die Bestände vieler Datenbanken Überschneidungen aufweisen, was sich - je nach Suchanfrage - in unterschiedlich großen Schnittmengen der Ergebnisse widerspiegelt. So können Treffer, die man über den Katalog einer Universitätsbibliothek erhält, ebenso von Fachdatenbanken geliefert werden, die ihre Informationen zum Teil aus Universitätsbibliotheken beziehen. In anderen Fällen sind einzelne Datenbanken sogar gänzlich in anderen, übergeordneten Datenbanken integriert. Zu letzteren zählen zum Beispiel Verbunddatenbanken, die die Bestände einzelner Bibliotheken unter einer gemeinsamen Oberfläche vereinen. Ein weiteres Beispiel sind Meta-Suchmaschinen, die die Bestände anderer Datenbanken zwar durchsuchen, selbst aber keine Datensätze haben.

Manche Datenbanken bieten lediglich eine Suche nach Titeln, während andere zusätzlich Abstracts oder sogar Volltexte durchsuchen. Mitunter sind Bestände auch verschlagwortet. Da aber keine international einheitlichen Richtlinien für Verschlagwortung existieren, kommt es bei einer Schlagwortrecherche zwangsläufig zu einer unterschiedlichen Interpretation ein- und derselben Suchanfrage durch unterschiedliche Datenbanken.

Datenbanken für die Literaturrecherche lassen sich wie folgt klassifizieren:

- Bibliotheksverbünde und ihre Verbunddatenbanken

- Meta-Suchmaschinen über Verbunddatenbanken und Bibliothekskataloge

- Wirtschaftswissenschaftliche Fachdatenbanken und spezielle Internetportale

- Zeitschriftendatenbanken

- Digitale Zeitschriftenarchive

- Datenbanken für Arbeitspapiere

- Wissenschaftliche und generelle Suchmaschinen im Internet 
Sind geeignete Datenbanken zur repräsentativen Stratifizierung des Literatursamples ausgewählt, beginnt die eigentliche Recherche, d.h. die Filterung relevanter Publikationen. Hierzu wird ein Suchwortkatalog definiert und nachfolgend auf die ausgewählten Datenbanken angewendet. Entscheidender Unterschied zu allen anderen Suchverfahren ist die Tatsache, dass zunächst alle Treffer zu jeder Suchwortanfrage grundsätzlich als relevant eingestuft und zur Literaturliste hinzugefugt werden. Eine subjektive Auswahl anhand meist wenig nachvollziehbarer Kriterien unterbleibt also.

\subsubsection{Generierung einer repräsentativen Literaturauswahl}

Ziel des zu erstellenden Literatursamples ist die repräsentative Abbildung der Literatur gleichgewichtsmodell-basierter Analysen des Weltagrarhandels im Rahmen der WTO Doha-Runde. Um die methodische Komponente dieser Fragestellung zu definieren, werden die Auswahlkriterien eines qualitativen Literaturüberblicks zu zeitgenössischen Simulationsmodellen (van Tongeren, van Meijl und Surry 2001) verwendet. Die thematische Komponente wird durch Schlüsselwörter aus $\$ 13$ der Ministererklärung im Rahmen der Doha Handelsrunde gebildet (WTO 2005), da diese Erklärung zu Beginn der Doha-Runde sowohl die seit Gründung der WTO wichtigsten Themen des Agrarhandels vereint, als auch die wichtigsten Stichworte für die wissenschaftliche Diskussion seit der Doha Konferenz geliefert hat. Anhang A (dort Tabelle 13) listet diese Suchworte auf. Wie unschwer zu erkennen ist, würden die meisten dieser Suchworte nicht explizit auf Publikationen im Bereich der angestrebten Literatur-Grundgesamtheit verweisen. Erst die Kombination der Suchbegriffe aus Tabelle 13 grenzt die angestrebte Literatur ein. Zusätzlich wird jeder Suchbegriff um das Kürzel „WTO“ ergänzt. Die 16 methodischen (Vektor N) und 10 thematischen (Vektor M) Such-worte bilden eine Matrix aus 160 möglichen Suchwortkombinationen $(\mathrm{N} \times \mathrm{M} \times$,wto“ $\times$,model“ $)$.

All diese Suchwortkombinationen wurden an die in Anhang A (dort Tabelle 12) genannten Datenbanken gesendet. Für jede dieser Datenbanken wurden alle zurückgegebe-nen Literaturverweise in ein Spreadsheet importiert und um Duplikate bereinigt. Das Ergebnis der Anwendung dieser Suchprozedur auf simulationsmodell-basierte Literatur im Rahmen der Doha-Runde ist ein Literatursample mit über 1200 Studien. Der Begriff „Studien“ drückt dabei aus, dass es sich nicht aus-schließlich um Publikationen auf dem Hintergrund eines wissenschaftlichen Begutachtungsprozesses handelt, sondern um Veröffentlichungen aus dem wis-senschaftlichen Umfeld allgemein.

Bei weitem nicht alle dieser 1200 Studien enthalten Ergebnisse eigener Simulationsexperimente, sondern beziehen sich vielmehr auf Simulationsergebnisse anderer Studien. Sowohl solche Sekundärpublikationen im Hinblick auf 
die Verwendung von Simulationsergebnissen als auch Duplikate, bei welchen identische Simulationsexperimente als Arbeitspapier, Konferenzbeitrag, etc. erscheinen, werden aus dem Datensatz eliminiert. Darüber hinaus existieren einige Studien, welche keine Wohlfahrtseffekte (bzw. Einkommensänderungen) ausweisen, sondern auf die Änderung von Handelsbilanzen, Preisen oder Produktionsmengen fokussieren. Diese Studien wurden zunächst nicht berücksichtigt, könnten aber eventuell Grundlage für weitere Meta-Analysen bilden, bei denen diese Variablen jeweils als abhängige Variablen betrachtet werden.

Ferner müssen Studien eliminiert werden, welche kaum oder keinerlei Hinweise auf das einer Simulation zu Grunde liegende Modell enthalten (beispielsweise beziehen sich manche Studien allgemein auf "Weltbank-Schätzungen“ oder auf „GTAP“). Eine letzte Gruppe ausgeschlossener Studien präsentiert Simulationsergebnisse nicht in numerischer Form sondern beispielsweise durch Grafiken, was eine präzise Quantifizierung der simulierten Wohlfahrtseffekte unmöglich macht.

Das Resultat dieses Filterns ist ein Literatursample von 230 Studien, welche über die Jahre 1994 bis 2006 publiziert wurden. All diese Studien präsentieren eigene Simulationsergebnisse, in deren Mittelpunkt die Ausweisung von Wohlfahrtsänderungen steht, welche in numerischer Form präsentiert werden. Ferner findet sich eine konkrete Beschreibung des experimentellen Aufbaus sowie des verwendeten Modells. Der mitunter geäußerte Vorwurf an Meta-Analysen, „Äpfel mit Birnen“ vergleichen zu wollen, kann an dieser Stelle ausgeschlossen werden, da alle 230 Studien innerhalb dieses Literatursamples quantitative Schätzungen über die Auswirkung von Handelsliberalisierung, gemessen in der einen oder anderen Spezifikation von Wohlfahrtsänderungen, ausweisen und alle Studien auf einem hinsichtlich seiner theoretischen Wurzeln identischen Modellierungsansatz beruhen. Folglich ist nicht ersichtlich, warum diese Studien keinem quantitativen Vergleich unterzogen werden sollten.

Die Repräsentativität des Literatursamples wurde stichprobenartig überprüft, indem die Literaturlisten qualitativer Reviews zu verwandten Themenbereichen mit dem Literatursample abgeglichen wurden. Charlton und Stiglitz (2005) sowie UNCTAD (2003) haben solche Überblicke vorgenommen; beide Studien wurden durch das Literatursample erfasst. Im Falle der Literaturliste von Charlton und Stiglitz (2005) zeigt sich, dass alle dort einem Vergleich unterzogenen Studien auch durch die hier verwendete Suchstrategie erfasst wurdenausgenommen Studien, deren Titel bereits $\mathrm{zu}$ erkennen geben, dass andere als Agrar- oder allgemeine Doha Themen im Mittelpunkt der Simulationen stehen; eine Ausgrenzung solch spezieller Studien war mit der Suchstrategie beabsichtigt, um die Informationsverarbeitungskosten zu verringern.

Dieser stichprobenartige Abgleich des Literatursamples mit der in einem begutachteten Journal veröffentlichten Literaturliste eines thematisch eng ver- 
wandten Surveys von Charlton und Stiglitz (2005) gibt folglich keinerlei Anlass, an Repräsentativität und Effektivität des Verfahrens zu zweifeln. Ein weiterer Vergleich mit der Liste von simulationsmodell-basierten Publikationen, welche die Autoren von UNCTAD (2003) vergleichend diskutieren, zeigt, dass zwei Studien (Anderson, Hoekman und Strutt 1999, ABARE 2000) gemessen an ihren Titeln einen hohen Grad an Relevanz für die vorliegende Fragestellung aufweisen, durch die Samplingstrategie aber nicht erfassten wurden.

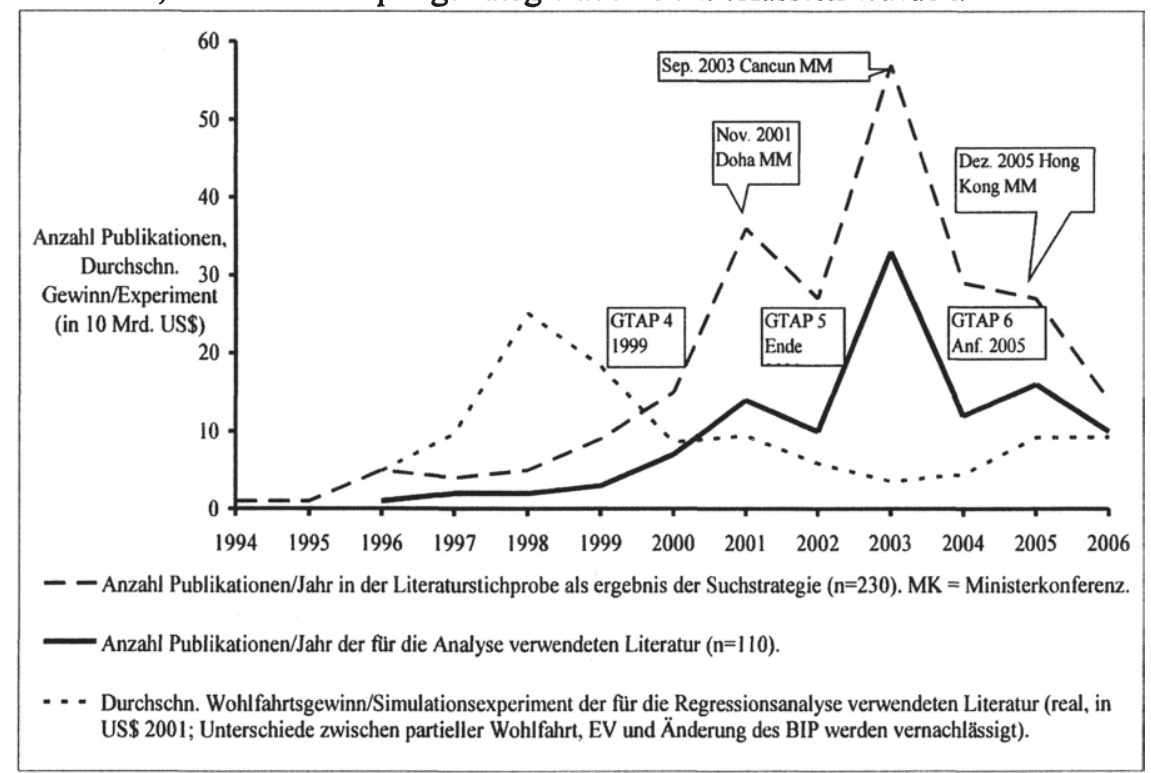

Abbildung 3: Das Literatursample.

Quelle: Eigene Zusammenstellung.

Insgesamt wurden 14 Publikationen mit eindeutig hoher Relevanz für die Fragestellung abgeglichen, wovon 2 durch das Literatursample nicht erfasst wurden. Eine dieser beiden Studien (Anderson, Hoekman und Strutt 1999) ist in einem im Internet nicht frei zugänglichen Journal erschienen und hätte im Rahmen der durchgefürten Suchanfragen z.B. durch EconLit oder OCLC Eco erfasst werden müssen. Es wird deutlich, dass sich trotz inhaltlicher Relevanz weder in Titel noch Abstract eine der verwendeten Suchwortkombinationen (siehe Anhang A) findet, was eine Erklärung dafür liefert, dass diese Studie, obwohl inhaltlich relevant, durch die verwendete Suchstrategie nicht erfasst wird. Ein solcher Informationsverlust innerhalb des Samples muss in Kauf genommen werden, um die Kosten der Informationsverarbeitung begrenzen zu können. Die Publikation des Australian Buro of Agricultural and Ressource Economics 
(ABARE 2000) ist ebenfalls themenrelevant, weist alle erforderlichen Schlüsselworte auf, ist im Gegensatz zu Anders.on, Hoekman und Strutt (1999) frei im Internet verfügbar und wurde ebenfalls durch die Suchstrategie nicht erfasst. Da es sich um einen Report handelt, ist diese Studie in keiner der abgefragten Datenbanken zu finden. Die Volltextsuche von Google hätte allerdings diese Publikation erfassen müssen; eine mögliche Erklärung kann darin bestehen, dass die Suchstrategie von Google evtl. den Bereich des Internets, in welchem ABARE (2000) angesiedelt ist, aufgrund seltener Aufrufe oder fehlender Verlinkung zu häufig aufgerufenen Seiten unberücksichtigt lässt (sog. „Deep Web“).

Das erstellte Literatursample konnte offensichtlich nicht alle relevanten Studien erfassen. Für die Repräsentativität des Samples ist dies unerheblich, so lange die Auslassungen zufällig sind. Wird allerdings deutlich, dass bestimmte Datenbanken oder Suchwortsynonyme bisher nicht erfasst wurden, und damit nicht nur einzelne Studien sondern ganze Bereiche der Literatur ignoriert werden, so kann das Sample unter Verwendung der gleichen Suchwortmatrix um eben diese Datenbanken problemlos ergänzt werden, was gegenüber den für Meta-Analysen in der Ökonomie meist ublichen explorativen Suchen einen entscheidenden Vorteil darstellt:

Während explorative Suche für Meta-Analysen im Bereich der Ökonomie bisher üblich ist (Stanley und Jarrell 1989, Alston, Chang-Kang, Marra, Pardey und Wyatt 2000, Stanley 2001), kann die vorgenommene Strategie genau dokumentieren, welche Literatur aus welchen Informationsquellen Eingang in die Analyse gefunden hat. Meta-Analysen oder Surveys mit explorativer Strategie hingegen präsentieren meist Positivlisten der eingeschlossenen Literatur, geben aber kaum nachvollziehbare Auskunft darüber, welche Informationsquellen unberücksichtigt blieben. Dieser Mangel an Transparenz bezüglich der Repräsentativität meta-analysierter Publikationen kann leicht zu einem Verlust an Glaubwürdigkeit von ökonomischen Meta-Analysen führen: Aufgrund der vielfältigen Überschneidungen aus Themen und Methoden im Bereich angewandter Ökonomie können „relevante“ von „nicht relevanten“ Studien nicht immer eindeutig separiert werden, wodurch sich Meta-Analysen unter Umständen gegen den Vorwurf empirischer Beliebigkeit wehren müssen (Wachter 1988).

Die hier vorgestellte Methode zur Generierung eines Literatursamples bietet ein Konzept zur Durchfuhrung einer solchen ex ante definierten Recherche. Durch eine Auswahl von Datenbanken, welche für unterschiedliche Publikationsformen repräsentativ sind oder besondere Themenbereiche abdecken, kann eine Repräsentativität der Literaturauswahl erreicht werden. Durch eine definierte Liste von Suchwortkombinationen und anschließende Vollerhebung aller Treffer je Datenbank wird ein Sample geschaffen, welches bei vertretbarem Aufwand, methodischer Transparenz und intersubjektiver Reproduzierbarkeit der tatsächlichen Grundgesamtheit an relevanter Literatur sehr nahe kommen 
kann. Außerhalb von Methoden zur Forschungssynthese kann das vorgestellte methodische Konzept in beliebiger Weise mit anderen Suchstrategien kombiniert werden und als Leitfaden zur systematischen Recherche angewandt-ökonomischer Literatur dienen.

Abbildung 3 zeigt das Literatursample im Überblick: Von den 230 Studien, wel-che als relevant identifiziert wurden, finden 110 Eingang in die MetaAnalyse. Diese Reduktion aufgrund fehlender Informationen in den betreffenden Studien erscheint jedoch im Hinblick auf das Publikationsjahr zufällig, sodass die Reprä-sentativität des Literatursamples nicht eingeschränkt wird. Der durchschnittliche Wohlfahrtsgewinn je Studie ist ebenfalls abgebildet, da einige Studien innerhalb der Literatur die Veränderung simulierter Wohlfahrtseffekte im Zeitablauf in ähnlicher Weise abgetragen und diskutiert haben („Trade Pessimism", vgl. z.B. Bouët und Krasniqi, 2006). Bei Ausweisung dieses Durchschnitts in Abbildung 3 ist jedoch weder für unterschiedliche Preisniveaus in den jeweiligen Studien noch für unterschiedliche Arten der Wohlfahrtsmessung (EV, Änderung des BIP, partielle Renten und Staatseinnahmen, etc.) kontrolliert, weshalb der Graph in Abbildung 3 nur eingeschränkte Aussagekraft besitzt.

\subsection{Vorstudien zur Meta-Analyse von Agrarsektormodellen}

Aufgrund der möglichen praktischen Schwierigkeiten sowie eventuell steigender Skalenerträge bei Datenerhebung und Umsetzung einer Meta-Analyse angewandter Gleichgewichtsmodelle wurden verschiedene Vorstudien anhand des Literatursamples durchgeführt, welche bei reduziertem Ressourceneinsatz Klarheit über Fragen des praktischen Vorgehens im Rahmen der eigentlichen Analyse schaffen und eine Konkretisierung der in Kapitel 1 formulierten Forschungsfragen erreichen sollten. Diese Vorstudien hatten folgende Ziele:

Vorstudie 1, Hess (2005a): Determination der typischerweise verfügbaren Informationen innerhalb der Studien des Literatursamples: Welche Ergebnisse werden ausgewiesen und welche Informationen über Politikexperiment, Modellannahmen sowie über verwendete Daten und Parameter werden typischer Weise dokumentiert?

Vorstudie 2, Hess (2005b) und Hess (2006): Möglichkeiten und Grenzen der ökonometrischen Meta-Modellierung werden anhand eines Subsamples ausgesuchter Studien überprüft. Zur Anwendung kommt sowohl ein lineares Re- 
gressionsmodell (OLS Schätzer) sowie alternative ökonometrische Repräsentationen durch ein Mixed Effects Modell (Maximum Likelihood Schätzer).

Vorstudie 3, Hess und von Cramon-Taubadel (2006, 2007a): In dieser dritten Pilotstudie werden die Ergebnisse aus Pilotstudie 1 und 2 zusammengefasst und es wird auf Erfahrungen aus diesen Vorstudien aufgebaut: Die bisher durchgeführten Analysen konnten nur anhand ausgewählter, besonders homogener Studien durchgeführt werden. Insbesondere für die abhängige Variable und für die sehr heterogenen Politikexperimente mussten einheitliche Bezugsgrößen als Approximation entwickelt werden, welche in dieser Vorstudie angewendet wurden. Ferner sollte überprüft werden, ob eine Ausweitung des gewählten Ansatzes auf die im Literatursample ebenfalls vorhandenen, partiellen Gleichgewichtsmodelle ausgedehnt werden kann. Zur Approximation der unterschiedlichen Politikexperimente wurde eine Referenzdatenbank entwickelt; zur Ergänzung fehlender Dokumentation der Simulationsexperimente wurde ein Internetsurvey mit Autoren durchgeführt.

\subsubsection{Zusammenfassung Hess (2005a)}

Insbesondere das GTAP-Netzwerk hat Offenheit und Transparenz zu einem seiner wichtigsten Prinzipien erhoben. Nicht zuletzt dadurch wurde der Kreis von CGE-Modellanwendern entscheidend vergrößert. Die meisten CGE-Modelle weltweit verwenden inzwischen die GTAP-Datenbank. Allerdings gibt es keine verbindlichen Standards, anhand derer Modellierer ihren Simulationsaufbau dokumentieren könnten. Die Vorstudie 1 nimmt eine explorative Erfassung der innerhalb des durchschnittlichen Papiers im Literatursample zur Verfügung stehenden Informationen über Modell, Daten und Simulationsaufbau vor. Aus dem Literatursample wurde eine Zufallsstichprobe von 50 Studien gezogen, anhand derer diese Informationen soweit sie zur Verfügung standen erfasst wurden. Die Regressionsanalyse führte im Folgenden dazu, dass die Hypothese, dass Dokumentation von Simulationsexperimenten über Studien hinweg uneinheitlich und lückenhaft ist, nicht verworfen werden konnte, was systematische Vergleiche von Simulationsexperimenten möglicherweise erschwert. Andererseits waren die zur Verfügung stehenden Informationen bereits ausreichend, um einen gewissen Anteil der Varianz innerhalb der abhängigen Variable systematisch erklären zu können, weshalb die Hypothese, dass die zur Verfügung stehende Information für einen systematischen quantitativen Vergleich ausreichen würde, ebenfalls nicht verworfen werden konnte. Aus den Ergebnissen wurde geschlossen, dass eine vollständige Dokumentation von Simulationsergebnissen eventuell nicht in allen Fällen notwendig ist, sofern bestimmte Schlüsselinformationen für jede Studie zur Verfugung gestellt werden. Vor- 
schläge für solche Schlüsselinformationen wurden in Vorstudie 1 anhand von Koeffizienten abgeleitet, welche sich in der verwendeten Regression als signifikant erwiesen.

Für den weiteren Projektverlauf wurde aus den Ergebnissen dieser Studie abgeleitet, dass die durchschnittlich zur Verfügung stehenden Informationen insgesamt lückenhaft sind. Um dem aus unzureichender Dokumentation für die Meta-Analyse möglicherweise entstehenden Messfehler $\mathrm{zu}$ begegnen, wurde daher als Erkenntnis aus Pilotstudie 1 die Durchfuhrung eines Internetsurveys mit Autoren von Studien innerhalb des Literatursamples beschlossen. Die technische Implementierung dieses Internetsurveys folgte Erkenntnissen aus Pilotstudie 1 und 2. Eine besondere Schwierigkeit lag in der Quantifizierung von Simulationsexperimenten, da nahezu jede Studie ihre eigene Aggregation von Ländern und Sektoren vornimmt, was zu unterschiedlichen Niveaus der Ausgangszölle in jedem Sektor führt.

\subsubsection{Zusammenfassung Hess (2005b) und Hess (2006)}

In Pilotstudie 2 und 3 standen Fragen der ökonometrischen Modellierung im Rahmen einer Meta-Analyse angewandter Gleichgewichtsmodelle im Vordergrund. Hierzu wurde auf einen ausgewählten Datensatz CGE-basierter Simulationsexperimente zurückgegriffen. Alle CGE-Studien in diesem Datensatz verwenden die GTAP-4- oder GTAP-5-Datenbank. Da noch keine Möglichkeit bestand, kompliziertere Politikexperimente in vergleichbarer Weise darzustellen, wurde die Analyse auf Simulationsexperimente reduziert, welche uniforme Reduktionen von entweder 50 Prozent oder 100 Prozent der Ausgangszölle vornehmen.

Der auf diese Art entstandene Datensatz war im Vergleich zu dem eigentlichen Literatursample und auch im Vergleich zu dem in Pilotstudie 1 verwendeten Sample hinsichtlich der Kriterien der verwendeten Ausgangsdaten, Modellansätze und Politikexperimente sehr homogen. Es gelang im Folgenden, einen sehr großen Teil der Varianz innerhalb der abhängigen Variable dieses Datensatzes (simulierte Änderung in äquivalenter Variation EV) durch die zur Verfugung stehenden Informationen statistisch zu erklären. Dabei wurde ein lineares Regressionsmodell (OLS-Schätzer) verwendet. Die Multikollinearität zwischen den erklärenden Variablen trat als Problem hervor (Test durch Konditionsindex $\kappa$ ) und machte deutlich, dass es notwendig ist, die in einer MetaAnalyse extrahierten Daten gemäß ihrer theoretischen Funktion innerhalb eines jeden Modells zu kategorisieren, wobei die gewählten Kategorien sta-tistisch so unabhängig voneinander wie möglich sein sollten. Heteroskedastizität zeigte sich ebenfalls innerhalb des Datensatzes (Diagnose durch Breusch-Pagan Test); es wurden robuste Standardfehler appliziert, um hierfür zu korrigieren. Ferner 
wurde alternativ zu dem linearen Modell mit fixen Effekten für den je-weils beteiligten Modellansatz eine Modellspezifikationen gewählt, welche der hierarchischen Struktur der Fehlerterme innerhalb des Meta-Datensatzes besser Rechnung trägt. Für dieses Mixt Effects Modell (ML-Schätzer) wurden ferner Kriterien der Modellselektion eruiert, welche den Vergleich der Schätzgüte von zwei Meta-Regressionsmodellen ermöglichen.

\subsubsection{Zusammenfassung Hess und von Cramon-Taubadel (2006, 2007a)}

Die dritte Pilotstudie fasst die Ergebnisse aus den beiden ersten Pilotstudien zusammen. Ziel war die Abbildung eines weitgehend repräsentativen Ausschnittes des Literatursamples in einer Meta-Analyse, weshalb der approximativen Standardisierung der Simulationsexperimente innerhalb des gesamten Samples Vorrang vor einer sehr präzisen Abbildung einzelner, aber evtl. nicht repräsentativ ausgewählter Simulationsexperimente gegeben wurde. Die für diesen Zweck entwickelte Referenzdatenbank konnte für wichtige Unterschiede zwischen nahezu allen Politikszenarien approximativ korrigiert werden.

Im Rahmen der dritten Pilotstudie wurde folgende Meta-Regression geschätzt: (Lineare Regression, OLS-Schätzer mit robusten Standardfehlern):

$$
Y=f(P, M, D)+\text { Fehlerterm }
$$

Die Vektoren $Y, P, M$ und $D$ wurden dabei wie folgt spezifiziert:

$Y=$ Outputvektor: Einbezogen wurden simulierte partielle Wohlfahrtsänderungen, simulierte Änderungen von GDP, sowie Äquivalente/Kompensierende Variation; Unterschiede wurden in der Regression durch Dummies abgebildet.

$\mathbf{P}=$ Politikvektor: Bei bivariaten Ergebnisvergleichen (vgl. Abbildung 1 und 2) wird häufig ignoriert, dass eine simulierte Reduktion des Zollsatzes $t$ um den Betrag $s$ mit $0<s \leq 1$ je nach Ausgangshöhe von $t$ eine sehr unterschiedliche absolute Reduktion darstellt. Der Inlandspreis eines Gutes $i$ in Region $r$ als Folge einer Zollreduktion sei:

$$
p_{r i}^{\text {Inland }}=p_{r i}^{\text {Grenze }}\left[1+t_{r i}\left(1-s_{r i}\right)\right]
$$

Mit: $p_{r i}=$ Preis Gut $i$ in Region $r ; t_{r i}=$ ad valorem Ausgangszoll auf Importe aus i; und $s_{r i}=$ Reduktion in $t_{r i}$.

Wie leicht zu erkennen ist, müssen für einen Vergleich von zwei Simulationen nicht nur die Zollveränderungen $s$ in allen Experimenten identisch sein („minus 50\% Zollabbau weltweit“, vgl. Abbildung 1 und 2), sondern auch die 
Ausgangszölle $t_{r i}$, um hinsichtlich eines Vergleichs weiterer Modellkomponenten von einem vergleichbaren Zollkürzungsexperiment sprechen zu können.

Um diesen Vektor der Politikänderungen über Simulationsstudien hinweg quantifizieren zu können, wurde eine Referenzdatenbank erstellt, durch welche die regionalen und sektoralen Aggregationen einer Simulation sowie die zugeordneten, angewandten MFN-Zölle für jede Simulation approximativ rekonstruiert werden können und einen Vergleich der tatsächlichen Höhe des Politikschocks ermöglichen.

Die genannte Referenzdatenbank erlaubt es ferner, jeder in einer Simulationsstudie vorkommenden, aggregierten Region zahlreiche makroökonomische Kenngrößen (GDP, Bevölkerungszahl, etc.) zuzuordnen und deren Zusammenhang mit simulierten Effekten ökonometrisch zu testen. Um dem Problem fehlender Daten zu begegnen, wurde ein Internet-Fragebogen an Autoren der Studie innerhalb des Samples versendet. Die Rücklaufquote dieses Surveys blieb jedoch unter 20 Prozent. Es stellte sich als Problem heraus, dass die Autoren einer Simulationstudie nicht notwendigerweise auch selbst die Simulation durchgefuihrt haben.

$M=$ Vektor der Modellspezifikationen:

Die große Abhängigkeit der Modellergebnisse von bestimmten, innerhalb der Literatur häufig vertretenen Modellspezifikationen konnte empirisch bestätigt und teilweise quantifiziert werden. Die wichtigsten Ergebnisse der Pilotstudie lassen sich wie folgt zusammenfassen:

Partielle Gleichgewichtsmodelle erreichen c.p. höhere Simulationsergebnisse als standardisierte CGEs (wie etwa das GTAP-Modell). CGEs mit höheren Armington-Elastizitäten als in GTAP verwendet weisen ebenfalls höhere simulierte Wohlfahrtsgewinne auf, in einer ähnlichen Größenordnung wie bei den partiellen Modellen, d.h. sehr hohe Armington-Elastizitäten nähern das Verhalten von Modellen ohne Armington-Annahme an.

Annahmen über steigende Skalenerträge und unvollkommenen Wettbewerb führen zu höheren simulierten Effekten als die in den meisten Modellen vorherrschende Annahme über vollkommenen Wettbewerb und konstante Skalenerträge auf allen Märkten.

Starken Einfluss besitzt der Grad der modellierten Kapitalakkumulation innerhalb von Handelsmodellen, also die Frage, ob der Kapitalstock eines Landes nach Liberalisierung wächst oder konstant bleibt (wachsender Kapitalstock = deutlich höhere Ergebnisse); dieser Effekt scheint auch der Hauptgrund für die höheren Simulationsergebnisse dynamischer Modelle zu sein, d.h. viele der betrachteten dynamischen Modelle weisen auch gleichzeitig einen im Zeitverlauf wachsenden Kapitalstock auf, während dynamische Modelle ohne diese Annahme keine höheren Gewinne ausweisen, sofern für unterschiedlich lange Simulationszeiträume korrigiert wird. 
Selten vorkommende Macro-Closures wie etwa die Johansen Macro-Closure (vgl. Rattsø 1982) sowie Annahmen über liberalisierte ausländische Direktinvestitionen sind mit weit überdurchschnittlich hohen Simulationsergebnissen assoziiert.

Die Modellierung von nicht-tarifären Handelshemmnissen (NTB) ist innerhalb der Literatur methodisch sehr uneinheitlich; die Ergebnisse solcher Studien streuen mit einer hohen Varianz.

D = Vektor der verwendeten Basisdaten:

Die große Bedeutung unterschiedlicher Basisdaten wurde bestätigt; insbesondere die GTAP-Datenbanken ermöglichen eine eindeutige Klassifizierung des verwendeten Datenausgangsmaterials. Im Falle partieller Gleichgewichtsmodelle wurde nur selten dokumentiert, aus welcher Quelle die verwendete Basisdaten stammen; für single country CGEs waren dies meist nationale InputOutput-Tabellen und Social Account Matrices (SAMs), welche auch häufig Eingang in die GTAP-Datenbank gefunden haben. Aufgrund dieser Teilweisen Heterogenität wurden GTAP-Datenbanken durch Dummy-Variablen unterschieden; weiter Datenquellen mussten aber anhand des Basisjahres als einzigem gemeinhin verfügbaren Merkmal gruppiert werden; innerhalb des Regressionsmodells zeigten sich für diese nicht-GTAP-Gruppierungen jedoch keine einheitlichen Effekte.

\subsubsection{Erhebung und Approximation der Meta-Daten im Überblick}

Dieser Abschnitt erläutert den technischen Ablauf bei der Datenerhebung. Eine genaue Definition der erhobenen Variablen erfolgt in Kapitel 3.6. Die Erhebung der Daten für den Meta-Datensatz wurde anhand eines in Abbildung 4 zusammengefassten Workflows durchgeführt. Der Meta-Datensatz besteht aus drei Hauptkomponenten: einer Tabelle mit Informationen zur abhängigen Variable, einer Tabelle mit Informationen über Modell-Charakteristika, Studienkontext und Datengrundlage und einer dritten Tabelle mit Proxy Variablen für die Höhe der durchgeführten Änderung politikrelevanter Variablen und Informationen über Bruttosozialprodukt, Bevölkerungsgröße, etc., welche den jeweiligen Observationen im Datensatz zugeordnet werden.

Aufgrund des in Gleichung (3.15) verdeutlichten Zusammenhangs können Simulationsergebnisse nicht sinnvoll miteinander verglichen werden, ohne dass sowohl für das Ausgangsniveau der in einer Studie verwendeten Protektion sowie für die simulierten Politikänderungen dieser Protektion kontrolliert wird. $\mathrm{Zu}$ diesem Zweck wurde eine Referenzdatenbank konstruiert (Ausführung in der open-source Software MySQL mit einem MS-Access Benutzerinterface), welche Informationen über angewandte und gebundene Zölle, Produktionswerte 
und Handelsströme enthält. Quellen für diese Datenbank sind GTAP 5 und GTAP 6, FAO, ATPSM und MacMap.

\begin{tabular}{|c|c|c|}
\hline \multicolumn{3}{|c|}{$\begin{array}{l}\qquad \text { Suchwortmatrix: } \\
\text { [Schlusselworte fur Modelle] x [Schlusselworte furr Doha Runde] }\end{array}$} \\
\hline \multicolumn{3}{|c|}{ Anwendung auf ausgewăhlte Literaturdatenbanken } \\
\hline \multicolumn{3}{|c|}{$\begin{array}{l}\text { Nach Anwendung der Filterkriterien: } \\
\text { Literatursample aus relevanten Publikationen }\end{array}$} \\
\hline $\begin{array}{l}\text { Abh. Variable: } \\
\text { Simulations- } \\
\text { ergebnisse auf } \\
\text { regionaler Ebene } \\
(\Delta, \text { Wohlfahrt') }\end{array}$ & $\begin{array}{l}\text { Fragebogen/Internetsurvey: } \\
\text { Extraktion der Informationen über } \\
\text { Modellcharakteristika, Datenbasis, } \\
\text { verwendete Parameter, Autoren, ... }\end{array}$ & $\begin{array}{c}\text { Referenzdatenbank: } \\
\text { Regionale/Sektorale } \\
\text { Aggregation jeder Studie; } \\
\text { simulierte Politikänderung } \\
\text { wird approximiert. }\end{array}$ \\
\hline$Z$ & ung der drei Datenbanken: Meta- & atz zur Analyse \\
\hline
\end{tabular}

Abbildung 4: Das System der Datenerhebung im Überblick. Quelle: Eigene Darstellung.

Hieraus wurden die regionalen und sektoralen Anordnungen jeder Pu-blikation nachaggregiert und den jeweils aggregierten volkswirtschaftlichen Da-ten (GDP, Bevölkerungszahl, etc.) der PENN World Tables (Heston, Summers, Aten 2006) zugeordnet.

\subsection{Meta-Analyse: Definition der Variablen}

Das verwendete Literatursample enthält wie in Kapitel 3.3.2 beschrieben 230 simulationsmodell-basierte Studien. Aus diesen 230 Studien konnten 110 Studien in den endgültigen Meta-Datensatz aufgenommen werden. Ca. 60 Studien wurden eliminiert, weil sie bei näherem Hinsehen nicht auf allgemeine, multilaterale Doha-Szenarien mit Schwerpunkt auf internationalen Agrarmärkten abzielen, sondern vielmehr verwandte Themen wie beispielsweise regionale Freihandelsabkommen, globale Auswirkungen von Technologieadap- 
tion, länderspezifische Aspekte eines WTO-Beitritts, sowie die technischen Aspekte der Modellierung von armutsreduzierenden Effekten der HandelsLiberalisierung auf Haushaltsebene analysieren. Weitere 60 Studien wurden nicht in das endgültige Sample aufgenommen, da die Dokumentation des experimentellen Aufbaus innerhalb dieser Studien zu lückenhaft war, um die Charakteristika der jeweiligen Simulationsexperimente hinreichend abbilden zu können.

Publikationen, welche einem wissenschaftlichen Begutachtungsprozess unterzogen wurden, sind meist transparenter und umfassender dokumentiert als andere Studien; im Fall nicht wissenschaftlicher Publikationen fällt ferner auf, dass in einigen Fällen selbst fundamentale Charakteristika des Experiments, wie beispielsweise der Umfang simulierter Politikänderungen oder fundamentale Charakteristika des verwendeten Modells keine Erwähnung finden.

Heterogene Dokumentationsstandards bedeuten für eine Meta-Analyse einen Trade-Off zwischen der Zahl der Studien, die in ein Literatursample aufgenommen werden kann einerseits, und der Zahl erklärender Variablen, welche quantifiziert und in der ökonometrischen Schätzgleichung repräsentiert werden können andererseits. Eine größere Zahl erklärender Variablen verringert zwangsläufig die Zahl der Studien, welche berücksichtigt werden können.

Ziel der hier durchgeführten Meta-Analyse ist die Identifikation von Modellcharakteristika und weiteren Faktoren, welche Simulationsergebnisse systematisch beeinflussen. Da jede Studie eine verhältnismäßig große Zahl an Observationen (durchschnittlich 50 je Studie) zum Datensatz beiträgt, kann durch Ausschluss der am schlechtesten dokumentierten Studien ein möglicher Messfehler innerhalb der erklärenden Variablen deutlich verringert werden.

\subsubsection{Definition der zu erklärenden Variable:}

Die abhängige Variable $\mathrm{Y}$ ist definiert als die simulierte Wohlfahrtsänderung $\Delta W$ innerhalb einer aggregierten Region $\mathrm{r}$ für ein Simulationsexperiment $\mathrm{e}$ innerhalb einer Studie $\mathrm{j}: Y_{r e j}=\Delta W_{r e j} ;$ mit $\mathrm{e}=1,2, \ldots, \mathrm{E} ; \mathrm{E}=$ Zahl der Simulationsexperimente im Meta-Datensatz, $r=1,2, \ldots, \mathrm{R}$ als Zahl der aggregierten Regionen und $s=1,2, \ldots, S$ als Zahl der Sektoren in jeder aggregierten Region r. Dabei gilt:

$$
\Delta W_{r e j}=\sum_{s=1}^{S} \Delta W_{s r e j}
$$

Jede Studie innerhalb des Literatursamples präsentiert demnach die Ergebnisse eines oder mehrerer Politikexperimente (Szenarien). Jedes dieser Experimente wiederum simuliert Wohlfahrtsänderungen $\Delta W$ in einem oder mehreren geo- 
graphischen Regionen $r$. Diese geografischen Regionen wurden als Bezugsebene gewählt, obwohl auch innerhalb jeder Region Simulationsergebnisse auf Sektorebene anfallen, welche jedoch für globale Politikszenarien nur in seltenen Fällen vollständig ausgewiesen werden. Stattdessen werden diese regionalen Wohlfahrtseffekte auf Ebene eines jeden Simulationsexperiments $e$ summiert, sofern diese Änderung der Weltwohlfahrt eines Simulationsexperiments nicht explizit ausgewiesen ist:

$$
\Delta W_{e j}=\sum_{r=1}^{R} \Delta W_{r e j}
$$

Öffentliche Diskussionen fokussieren typischerweise auf simulierte Wohlfahrtsänderungen als Indikator zu erwartender Folgen von Liberalisierungsschritten, aber bei näherem Hinsehen werden sehr unterschiedliche Messgrößen für diese Wohlfahrtsänderungen verwendet. Innerhalb des Literatursamples sind sowohl äquivalente und kompensierende Variation (EV, CV), simulierte Änderungen der absoluten Höhe des Bruttosozialproduktes, prozentuale Änderungen des Bruttosozialproduktes, sowie partielle Wohlfahrtsänderungen beispielsweise als Summierung von Renten vertreten.

All diese simulierten Änderungen der Bezugsgröße „Wohlfahrtsänderung“ wurden in Millionen US-Dollar umgewandelt und anhand entsprechender Wechselkurse und Deflationierungsfaktoren auf das Basisjahr 2001 umgerechnet. Für die verbleibenden Unterschiede zwischen diesen Wohlfahrtsmaßen wurden Dummy-Variablen eingeführt. Dabei wird unterstellt, dass die Unterschiede zwischen diesen Messgrößen einem linearen Zusammenhang folgen.

Dynamische Modelle geben Aufschluss über den Entwicklungspfad von simulierten Wohlfahrtsänderungen innerhalb einer Volkswirtschaft während des betrachteten Simulationszeitraums. Diese Informationen konnten nicht nutzbar qualifiziert werden. Stattdessen wurde verglichen, ob Simulationsergebnisse dynamischer Szenarien für das letzte Jahr des Simulationszeitraums, als Durchschnitt des Simulationszeitraums oder als Integral unter dem betrachteten Simulationszeitraum ausgewiesen waren. Sofern dynamische Gewinne ausgewiesen waren und dies relativ zu einer sich entwickelnden BSP-Baseline geschah, wurde diese Baseline durch angenommene 2 Prozent BSP-Wachstum in jedem Land approximiert. Tabelle 2 und Tabelle 5 fassen deskriptive Statistiken und Definitionen der verwendeten Variablen zusammen. Diese Approximation ist möglicherweise zu ungenau; eine Implementierung häufig verwendeter BSP-Wachstumsraten, (meist Projektionen der Weltbank) in die Referenzdatenbank konnte jedoch bisher nicht realisiert werden.

Die Definition einer Beobachtung von $Y_{i}=Y_{\text {rej }}$ innerhalb des Meta-Datensatzes (mit $i=1,2, \ldots, N ; N=$ Zahl der Beobachtungen im Meta-Datensatz) hat 
zwei wichtige Konsequenzen: Da die durchschnittliche Studie mehr als nur ein Liberalisierungsexperiment $e$ durchführt und durchschnittliche Modelle mehr als nur eine Region $r$ bzw. ein Land abbilden, ergeben sich aus 110 verwendeten Studien weit mehr als 110 Observationen (Tabelle 1). Aus diesem Grund erschien die Einfuhrung von Gewichtungsfaktoren notwendig: Da das erklärte Ziel in einer Quantifizierung wichtiger Einflussgrößen auf simulierte Ergebnisse besteht, könnten besonders einflussreiche Spezifikationen, wie beispielsweise unterschiedliche Macro-Closures, nicht adäquat gemessen werden, da sie mit einer niedrigen Zahl an Observationen im Datensatz repräsentiert sind. Aus diesem Grund wird die Meta-Regression (Gleichung 3.14) in Tabelle 3 und in Tabelle 4 in Form einer gewichteten sowie einer ungewichteten Variante abgebildet.

\subsubsection{Definition der unabhängigen Variablen}

Als Resultat des Datenerhebungsprozesses anhand des Workflows (Abbildung 4) wurden ca. 150 unterschiedliche Variablen erfasst, wovon viele kollinear sind. Daraus ergab sich das Problem, ein übersichtliches und theoretisch konsistentes Set unabhängiger Variablen für die Schätzgleichung selektieren zu müssen. Um für diesen Prozess Ausgangspunkte zu identifizieren, wurde ein Literaturüberblick von Modellierungsmethoden und Modellvergleichen erstellt, um Schlüsselvariablen und Einflussgrößen zu identifizieren, welchen Experten starken Einfluss auf die Simulationsergebnisse zuschreiben und welche aus handelstheoretischer Sicht von besonderem Interesse sind (vgl. Hertel 1999; Hertel spricht von "model pre-selection").

Neben dieser explorativen Analyse wurde auch Stepwise Regression und Regression Trees (Breiman et al. 1984) verwendet. Diese Analyse hat wichtige unabhängige Variablen identifiziert, welche in der Literatur diskutierte Modelleinflusse repräsentieren und welche einen signifikanten Einfluss auf die abhängigen Variablen in der Schätzgleichung haben.

Modellcharakteristika (Vektor $M$ in Gleichung 3.14) - Diese allgemeine Kategorien umfasst folgende Variablen:

Handelt es sich bei dem Modell um ein partielles oder ein allgemeines Gleichgewichtsmodell? PE-Modelle (Dummy $=1$ für PE, andernfalls 0) unterstellen, dass die betrachteten Märkte nicht durch Wechselwirkungen mit anderen Märkten einer Volkswirtschaft beeinflusst werden und diese Wechselwirkungen folglich unberücksichtigt bleiben können. Aus diesem Grund kann erwartet werden, dass der PE-Dummy ein positives Vorzeichen aufweisen wird, da viele Wechselwirkungen innerhalb des Modells sich tendenziell aufheben werden.

Gleichzeitig sind viele PE-Wohlfahrtseffekte aber auch in Form summierter Renten (Produzentenrente, Konsumentenrente, Staatsausgaben) gemessen und betreffen daher nur einen Ausschnitt einer Volkswirtschaft. Eine Diskussion 
der theoretischen Zusammenhänge zwischen Veränderung der Konsumentenrente und EV findet sich bei Mas-Colell, Whinston und Green (1995). Unterschiedliche Wohlfahrtsmaße im Angesicht von Ausgangsprotektion sind bei Martin (1997) dargestellt. Diese theoretischen Analysen legen insgesamt nahe, keine signifikanten Unterschiede zwischen partiellen Wohlfahrtsmaßen und EV zu erwarten. Diese Variablen sind innerhalb des Datensatzes jedoch mit dem Dummy für partielle Gleichgewichtsmodelle korreliert. Einige partielle Modelle weisen jedoch statt partieller Wohlfahrtsmaße EV aus, weshalb eine Aufnahme dieser Variable in die Regression notwendig wurde.

Macro-Closures sind notwendig, um der Tatsache $\mathrm{zu}$ begegnen, dass Modelle nicht mehr Variablen als Gleichungen enthalten können; im Rahmen einer Closure müssen daher einzelne Variablen exogen gehalten werden. Alle Modelle innerhalb des endgültigen Literatursamples nehmen entweder an, dass Ersparnisse gleich Investitionen sind (neoklassische Macro-Closure) oder dass Investitionen konstant sind und der Konsum endogen variiert. Die Literatur über Macro-Closures und ihren Einfluss auf Simulationsergebnisse weist keine generellen Effekte hinsichtlich einer Wirkung der Johansen-Macro-Closure im Vergleich zu anderen Macro-Closures außer in sehr modell-spezifischem Kontext aus.

In einigen Studien wird angenommen, dass die Handelsbilanz eines Landes oder mehrerer Länder fixiert ist. Wird diese Annahme getroffen, kann in ein Land als Folge von Liberalisierung kein zusätzliches ausländisches Kapital einfließen, um eine ausgeglichene Handelsbilanz zu gewährleisten. Folglich sollte der Dummy für eine fixierte Handelsbilanz (Dummy $=1$ wenn ja) auf niedrigere Wohlfahrtseffekte verweisen (vgl. McDonald und van Tongeren 2003).

Manche Modelle weisen zusätzlich zur Annahme eines fixierten Angebots an Land und Arbeit weitere Restriktionen von Primärfaktoren auf. Hertel (1989) betont, wie wichtig es ist, zwischen unterschiedlichen Arten der Modellierung des Angebotsverhaltens zu unterscheiden. Fehlende Informationen einiger Studien und die sehr heterogene Spezifikationen der Angebotsseite innerhalb des Literatursamples machen es allerdings schwierig, eine diesem Zusammenhang angemessene Variable zu definieren. Die hier verwendete DummyVariable ist eine sehr allgemeine Kategorien für Spezifikationen, welche das Angebotsverhalten über den oben erwähnten Standard (Arbeit und Kapital in jeder Region fix) hinaus begrenzen. Solche erweiterten Annahmen beziehen sich auf die sektorale Faktormobilität, Spezifikationen des Arbeitsangebots sowie zusätzliche Politikrestriktionen (Quoten).

Zehn Dummy-Variablen wurden eingesetzt, um die Effekte von vier zentralen Modellcharakteristika sowie ihrer Interaktionsterme abzubilden. Diese vier Charakteristika sind folgendermaßen spezifiziert, wobei das jeweils zweitge- 
nannte Modellcharakteristikum als Dummy in der Regression den Wert eins annimmt:

1. Die Annahme von vollkommenem Wettbewerb gegenüber einem Modell mit unvollkommenem Wettbewerb.

2. Die Annahme von konstanten gegenüber steigenden Skalenerträgen: In einigen Modellen sind bestimmte Sektoren, meist Industrie und evtl. auch Dienstleistungen, niemals primäre Agrarsektoren, durch steigende Skalenerträge (einhergehend mit der Annahme von unvollkommenem Wettbewerb) abgebildet. Diese Simulationen sollten mit höherem Wohlfahrtsgewinn assoziiert sein.

3. Konstanter gegenüber wachsendem Kapitalstock: In einigen Simulationsexperimenten (dynamische Modelle, aber auch komperativ-statische Modelle mit besonderer Closure) nimmt der vorhandene Kapitalstock während eines Simulationsexperiments $\mathrm{zu}$; in anderen Simulationsexperimenten wird er konstant gehalten.

4. „Niedrige“ gegenüber „hohen“ Armington-Elastizitäten: Die Höhe der verwendeten Armington-Elastizitäten wird mangels detaillierterer Informationen hier als „niedrig“ klassifiziert, sofern Standard-GTAP-Werte oder sogar niedrigere Armingtons verwendet werden. Ein Simulationsexperiment weist „hohe“ Armington-Elastizitäten auf, sofern die verwendeten Werte größer als die Standard-GTAP-Werte sind (beispielsweise werden diese Standard-Werte in einigen Studien verdoppelt, vgl. Gehlhar, 1997).

Sechzehn Kombinationen dieser vier Variablen sind möglich, aber einige tauchen nur selten oder überhaupt nicht innerhalb des Literatursamples auf, sodass nur zehn Interaktionsdummies in der Regressionsgleichung erscheinen.

Dynamische Modelle: Die Länge des Simulationszeitraums in Jahren wurde als erklärende Variable aufgenommen und zwar sowohl in linearer als auch in quadratischer Spezifikation. Dynamische Studien innerhalb des Samples bilden Zeiträume von wenigen Jahren bis zu einem Vierteljahrhundert ab. Man kann erwarten, dass längere Simulationszeiträume c.p. auch größere Wohlfahrtsgewinne aufweisen, aber es ist sehr wohl möglich, dass dieser Zusammenhang nicht-linear ist, weshalb der quadratische Term aufgenommen wurde; die statischen Modelle weisen für diese Variablen den Wert Null auf.

Im Falle einiger Simulationen wird die Datenbasis vor Beginn des eigentlichen Simulationsexperiments in ein neues Basisjahr projiziert. Die Länge dieses Projektionzeitraumes (Jahre) wurde ebenfalls in die Schätzgleichung aufgenommen. Man kann davon ausgehen, dass solche pre-simulativen Projektionen mit niedrigeren simulierten Wohlfahrtseffekten einhergehen, da solche Projektionen häufig bereits einige Liberalisierungsschritte im Vorfeld einer Simulation inkorporieren und nicht mehr dem eigentlichen Simulationsexperiment zuordnen. Eine Doha-Simulation im Jahr 2000 könnte beispielsweise ins 
Jahr 2006 projiziert werden und antizipieren, dass die Agenda 2000 Reform in der EU bereits implementiert wurde. Je mehr Liberalisierungsschritte bereits vor der eigentlichen Simulation durchgeführt worden sind, um so niedriger wird als Folge der Simulationsgewinn ausfallen.

\section{Daten und Parameter: (Vektor $D$ in Gleichung 3.14)}

Die Aggregierung der im jeweiligen Modell verwendeten Datenbasis in Regionen, Länder und Produkte spielt eine entscheidende Rolle. Drei Variablen wurden aufgenommen: Einerseits die Zahl der Regionen und Zahl der Sektoren innerhalb einer Simulation (nebst ihrem Interaktionseffekt) sowie die Zahl der explizit ausgewiesenen Agrarprodukte insgesamt. Die Agrarprodukte werden hier insbesondere betrachtet, da sie eine zentrale Rolle im Rahmen der DohaAgenda spielen und viele Studien innerhalb des Literatursamples, insbesondere PE-Gleichgewichtsmodelle, explizit auf Agrarsektoren fokussieren. Die Aggregation von Zöllen und Produkten ist ein wichtiges und fundamentales methodisches Problem im Bereich der angewandten Handelsmodellierung. Martin, van der Mensbrugghe und Manole (2003) trennen diesen Aggregations-Bias in ein Durchschnittsproblem und ein Gewichtungsproblem. Das Durchschnittsproblem resultiert aus der Tatsache, dass die Summe der Wohlfahrtsverluste als Resultat eines hohen $\left(t_{1}\right)$ und eines niedrigen $\left(t_{2}\right)$ Zolles in ansonsten identischen Märkten größer als die Summe der Wohlfahrtsverluste als Resultat eines Durchschnitts dieser beiden Zölle $\left(1 / 2\left[t_{1}+t_{2}\right]\right)$ ist. Das Gewichtungsproblem resultiert aus der Tatsache, dass die gemeinhin verwendeten Gewichtungsschemata anhand von Handelsmengen die Bedeutung hoher Zölle und im Extremfall prohibitiver Zölle unterschätzen. Aus diesem Grund ist zu erwarten, dass zunehmende Disaggregation zu höheren simulierten Wohlfahrtseffekten führt. Brockmeier und Pelikan (2006) verweisen in diesem Zusammenhang auf die methodischen Schwierigkeiten bei der Modellierung bestimmter formelbasierter Zollkürzungsszenarien (z.B. ,Schweizer Formel“).

Die verwendeten Parameter sind nicht für jede Simulation zweifelsfrei quantifizierbar. Vielmehr müssen vergleichsweise allgemeine Kategorien durch Dummy-Variablen abgebildet werden. Ein Dummy erfasst, wenn Autoren insbesondere darauf verweisen, dass sie bestimmte Parameter gemäß eigenem Dafürhalten modifiziert haben; ein anderer Dummy nimmt den Wert 1 an, wenn Autoren angeben, dass sie einige oder alle Parameter innerhalb ihres Modells ökonometrisch selbst geschätzt haben.

Die eigentlichen Liberalisierungsexperimente können nur mit Schwierigkeiten quantifiziert werden. Ein Teil der Verwirrung hinsichtlich der Unterschiede zwischen Simulationsexperimenten mag entstehen, weil wichtige Unterschiede zwischen dem, was zwei identische Liberalisierungsexperimente zu sein scheinen, übersehen werden (Gleichung 3.15). Während zwei Experimente, die beispielsweise beide einen 50-prozentigen Zollabbau auf Agrarprodukte in 
OECD-Ländern simulieren, identisch erscheinen mögen, können diese sich jedoch hinsichtlich der Art der verwendeten Zölle (gebundene gegenüber angewandten, präferentielle Handelsabkommen versus MFN-Zölle, etc.) gravierend voneinander unterscheiden (siehe Ergebnisse der Vorstudie 3).

Die Liberalisierungsexperimente wurden anhand dieser Referenzdatenbank auf folgende Weise quantifiziert:

Zollkürzungsexperimente wurden approximiert, indem regionale und sektorale Aggregationen für jede einzelne Simulationsstudie und jedes Simulationsexperiment innerhalb der Referenzdatenbank nachaggregiert und MFN ad valorem Ausgangszölle jedem Sektor zugeordnet wurden. Jeder ad valorem Zoll wurde dann mit dem Produktionswert des betreffenden Sektors in dem betreffenden Land (ausgedrückt in Millionen US-Dollar 2001) sowie den simulierten proportionalen Zollkürzungen multipliziert; das Ergebnis wurde für alle Produkte in allen Regionen aufsummiert. Auf diese Weise wurde für jedes der aufgrund unterschiedlicher Sektorgrößen und Ausgangszollsätze eigentlich nicht vergleichbaren Simulationexperimente in jeder aggregierten Region eine standardisierte Größe in Geldeinheiten ermittelt, welche Unterschiede zwischen dem Umfang des jeweiligen Liberalisierungsexperiments abbildet. Auf ähnliche Weise wurden simulierte Änderungen von Exportsubventionen und 'Amber' Box Agrarsubventionen über den zu Grunde liegenden Produktionswert approximiert. Negative Werte dieser Proxy-Variablen weisen auf eine Reduktion der Produktion hin, sodass die entsprechenden Regressionskoeffizienten ebenfalls ein negatives Vorzeichen haben sollten, sofern mit ihnen ein steigender Wohlfahrtsgewinn assoziiert ist. Diese Variable wurde ebenfalls in quadratischer Form in die geschätzte Regressionsgleichung (3.14) eingefügt, um für den nicht linearen Zusammenhang zwischen Wohlfahrtseffekten und zollbedingten Preisdifferenzen (meist zwischen Inlandspreis und Weltmarktpreis) zu korrigieren. Es muss darauf hingewiesen werden, dass diese Variable nicht in der Lage ist, sehr disaggregierte Handelsliberalisierungsszenarien und ausdifferenzierte Reformen der inländischen Agrarpolitiken abzubilden, wie dies in einzelnen Studien auf HS6 Ebene geschieht.

Wenn Autoren darauf verweisen, dass ein Liberalisierungsexperiment Daten über Non-Tariff-Barriers (NTBs) enthält, wurde die entsprechende ProxyVariable als [Produktionswert * 2 * MFN-Zoll * simulierte proportionale Änderung der NTBs] vorgenommen. Diese zweifelsohne sehr ungenaue Approximation wurde gewählt, weil nur wenige Studien eine NTB-Reduktion analysieren und kaum ausreichende Information darüber zur Verfügung stellen, wie die NTB-Ausgangsniveaus quantifiziert und in a.v. Beträge umgerechnet (,tariffiziert") wurden, sodass eine genauere Quantifizierung über Studien hinweg unmöglich ist. Die wenigen Studien, welche detaillierte Informationen darüber enthalten, wie NTBs geschätzt werden, verweisen darauf, dass die ermittelten 
Protektionsniveaus um einiges höher ausfallen als die korrespondieren MFNZölle. Dazu kommt, dass viele Experten der Meinung sind, dass die Wich-tigkeit von NTBs systematisch unterschätzt wird, da bisherige GATT- und WTOVerhandlungen sich vornehmlich auf eine Reduzierung konventioneller Zölle beschränkt haben. Es wird daher angenommen, dass die NTB-Daten in ei-nem Simulationsexperiment doppelt so hoch sind wie die vorliegenden MFNZollsätze. Folglich werden negative Werte dieser Variable darauf hinweisen, dass auch der $\mathrm{zu}$ erwartende Koeffizient ein negatives Vorzeichen aufweisen wird.

Änderungen in ,Blue ${ }^{6}$ und ,Green' Box Politiken der Landwirtschaft werden durch [Produktionswert * proportionale Änderung der Politik] approximiert. Dies wird einer detaillierten Repräsentation von ,Blue“ und ,Green“ Box Politiken in einigen PE Modellen nicht vollkommen gerecht, aber es ist eine realitätsnahe Approximation der Darstellung dieser Politiken in vielen CGE Modellen. ,Blue' Box Politiken sind c.p. weniger wohlfahrtsreduzierend als ,Amber' Box Politiken. Die ,Green' Box Politiken sind entweder wohlfahrtsneutral oder wohlfahrtssteigernd durch Produktivitätssteigerungen der Investitionen in Forschung und Entwicklung, obwohl die wohlfahrtsverringernden Auswirkungen einer Steuer berücksichtigt werden müssen, welche erhoben wird, um ,Green Box' Maßnahmen zu finanzieren. Folglich könnte der Effekt einer solchen Variable größer oder kleiner als 1 sein und es zeigt sich außerdem ein gewisses $\mathrm{Ma} ß$ an Kollinearität zwischen diesen Variablen und den reinen Änderungen von a.v. Zöllen, da simulierte Veränderungen von ,Blue‘ Box und ,Green' Box Politiken häufig einhergehend mit Zolländerungen simuliert werden (z.B. bei einer simulierten Umwandlung von Agrarzöllen in ,Green' Box Maßnahmen).

Zusätzlich zu den Variablen, welche das Liberalisierungsexperiment quantifizieren, gibt es einige Simulationen, welche einen Schock der Technologie oder ähnlicher Parameter, welche die Produktivität innerhalb eines Sektors beeinflussen, simulieren. Dieser technologische Fortschritt wird als proportionale Änderung der Technologie multipliziert mit dem Produktionswert eines Sektors gemessen. Solche Schocks sollten selbstverständlich mit steigenden Wohlfahrtsgewinnen assoziiert sein.

Das gesamte Handelsvolumen eines Landes oder einer Region für alle Güter und Dienstleistungen ist als erklärende Variable aufgenommen, soweit es im Modell und nicht nur innerhalb des Politikexperiments abgebildet wurde. Auf diese Weise wird für mögliche ToT-Effekte kontrolliert, welche in Sektoren entstehen können, die nicht unmittelbar simulierte Politikänderungen erfahren. Ferner können steigende Handelsvolumina mit größerer Marktmacht im internationalen Handel und womöglich auch mit größerer Verhandlungsmacht innerhalb von Handelsrunden einhergehen, wodurch größere Handelsnationen sich 
bessere Verhandlungspositionen sichern könnten, sodass die diskutierten Liberalisierungsszenarien möglicherweise größere Länder begünstigen. Die Variable kann einige, aber nicht alle Charakteristika einer geographischen Region erfassen, welche ihre ToT-Veränderungen in unterschiedlichen Simulationsexperimenten abbilden. Aus diesem Grund werden alternativ zu dieser Variable auch Dummies für jede aggregierte Region eingesetzt.

Die verwendeten Basisdaten sind in der Schätzgleichung durch vier Dummy-Variablen repräsentiert. GTAP 3 mit Basisjahr 1992 ist die Referenzdatenbasis, relativ zu welcher der Einfluss aller anderen Basisdaten gemessen wird. Dummy-Variablen für GTAP 4, GTAP 5 und GTAP 6 sowie alle anderen Basisdaten, beispielsweise nationale I-O Statistiken oder Produktions- und Handelsstatistiken, werden durch einen gemeinsamen Dummy abgebildet. Im Zeitverlauf enthalten jüngere Basisdaten niedrigere Protektionsraten und eine verbesserte Abbildung angewandter Zölle, insbesondere durch die MacMapDatenbank. Aus diesem Grund kann man erwarten, dass jüngere Basisdaten c.p. niedrigere Wohlfahrtseffekte ausweisen als Simulationsergebnisse auf Grundlage von GTAP 3.

Um Einflüsse des institutionellen Kontextes abzubilden, innerhalb dessen eine Simulationsstudie erstellt wurde, verwenden einige Meta-Analysten bibliografische Informationen über Studien (Publikationsjahr, Zahl der Autoren, etc.) sowie Informationen über das institutionellen Umfeld innerhalb dessen eine Studien entstanden ist. Im Falle der hier durchgeführten Meta-Analyse können viele dieser Variablen nicht zuverlässig quantifiziert werden. Die 25 innerhalb des Literatursamples am häufigsten genannten Autoren sind in 176 der 230 Publikationen involviert (entspricht 76 Prozent), was auf die Stärke von Netzwerken in der angewandten Handelsmodellierung hindeutet. Dadurch ergibt sich gleichzeitig die Schwierigkeit, das institutionelle Umfeld nicht eindeutig quantifizieren zu können: Der gleiche Professor kann beispielsweise einer Universität angehören, mag andernorts Working Papers veröffentlicht haben und veröffentlicht im Auftrag nationaler oder internationaler Institutionen Beratungspapiere und Gutachten. Es zeigte sich, dass die Variablen des wissenschaftlichen Kontextes, welche quantifiziert werden konnten (Publikationsjahr, Zahl der Autoren, Peer Review ja oder nein) keinen signifikanten Einfluss auf die Schätzung der abhängigen Variablen haben.

Im Fall des Publikationsjahres ist dies mit hoher Wahrscheinlichkeit auf Kollinearität mit den Basisdaten-Dummys zurückzuführen. Ferner ist es wahrscheinlich, dass der institutionelle Kontext durch die Variablen des Modellkontextes beeinflusst wird. Wenn beispielsweise Autoren für eine bestimmte Institution arbeiten und größere oder kleinere Wohlfahrtsschätzungen ausweisen möchten, so wird dies in einer Auswahl von Modellvariablen reflektiert sein und 
weniger ein unmittelbares Resultat der Zugehörigkeit zu einer bestimmten Forschungseinrichtung darstellen.

Eine weitere exogene Variable, die in die Schätzgleichung aufgenommen wurde, ist die Erfahrung der Autoren im Bereich angewandter Modellierung (gemessen in Jahren), da geringe Erfahrung für eine Reihe von Ausreißern innerhalb des Datensatzes verantwortlich sein könnte. Unglücklicherweise gibt es keine Möglichkeit, diese Informationen aus den Studien innerhalb unseres Samples zu isolieren und das verwendete Internetsurvey brachte diesbezüglich keinen ausreichenden Rücklauf an Antworten.

Zusammenfassend kann festgestellt werden, dass 34 erklärende Variablen ausgewählt wurden, welche wichtige Modellcharakteristika, typische Aspekte von Liberalisierungsexperimenten sowie häufig verwendete Basisdaten quantifizieren. Die Einführung weiterer erklärender Variablen führt nur begrenzt zu zusätzlichem Erkenntnisgewinn aufgrund von Kollinearitäten und uneinheitlicher Dokumentation in vielen Studien. Viele der hier verwendeten erklärenden Variablen sind zwangsläufig Approximationen einer größeren Zahl komplexer Modellcharakteristika, wovon einige nur ungenau abgebildet werden können. Die Analyse könnte auf Studien beschränkt werden, welche vollständig dokumentierte und weniger fragwürdig miteinander vergleichbare Simulationsexperimente enthalten, aber dies würde die Zahl verwendbarer Studien in dem verbleibenden Sample extrem einschränken und auf ein wahrscheinlich nicht mehr repräsentatives $\mathrm{Maß}$ reduzieren.

Der folgende Abschnitt zeigt, dass trotz dieser Bedenken das gewählte Set erklärender Variablen plausible und informative Schätzungen produziert. 
Sebastian Hess - 978-3-631-75694-2

Downloaded from PubFactory at 01/11/2019 02:52:17AM

via free access 


\section{Ergebnisse der Meta-Analyse}

In diesem Kapitel werden Ergebnisse der durchgeführten Meta-Analyse auf Basis des in Appendix A dokumentierten Literatursamples (vgl. auch Abbildung 3) vorgestellt und diskutiert. Die zuvor in Kapitel 3.6.1 definierte abhängige Variable wird im Rahmen eines linearen, multivariaten Regressionsmodells durch die in Kapitel 3.6.2 erläuterten Kovariate erklärt. Hinsichtlich der in Kapitel 3.2 beschriebenen möglichen Komplikationen bei der ökonometrischen Schätzung eines solchen Meta-Modells wird statistisch getestet und sofern notwendig korrigiert. Das ökonometrische Meta-Modell wird verwendet, um 1.) die innerhalb eines Simulationsexperiments insgesamt errechneten Gewinne und Verluste eines Szenarios zu erklären, also beispielsweise die regionalen Wohlfahrtsänderungen als Resultat des Harbinson-Proposals summiert für alle Regionen (nachfolgend „Weltwohlfahrtsänderung") und 2.) sollen die regionalen Wohlfahrtsänderungen in den verschiedenen geographischen Regionen innerhalb des Literatursamples erklärt werden (nachfolgend ,regionale Wohlfahrtsänderung"), beispielsweise die als Folge von Politikänderungen simulierten Wohlfahrtseffekte in den USA, der EU, Mauritius, etc.

\subsection{Deskriptive Auswertung des Meta-Datensatzes}

Abbildung 5 zeigt die simulierten regionalen Wohlfahrtseffekte (Mio. US-Dollar zu Preisen von 2001) innerhalb des Meta-Datensatzes in Region $r$. Die Abszisse zeigt die anhand einer Identifikationsnummer (ID) benannten Simulationsexperimente, d.h. die Lage eines Simulationsexperiments auf der Abszisse ist rein nominal skaliert.

Auffällig an Abbildung 5 ist die sehr unterschiedliche Varianz simulierter Wohlfahrts-veränderungen innerhalb der einzelnen Simulationsexperimente, wobei wenige einzelne Werte sehr weit vom Mittelwert des Samples entfernt sind, was nahe legt, sowohl Ausreißer als auch Heteroskedastizität zu vermuten und hierauf zu testen (siehe Kapitel 3).

Anhand dieses Meta-Datensatzes wurde zunächst eine Regressionsschätzung von Gleichung 3.14 vorgenommen. Unter Verwendung von Cook's Distance $\mathrm{Maß}$ (siehe Kapitel 3.2.4) weisen bestimmte Beobachtungen simulierter Wohlfahrtseffekte in einzelnen Regionen sehr hohe Werte für Cook's Distance auf; diese Beobachtungen entstammen überwiegend dem Michigan Model of Trade Modell (BDS), mit Ausnahme von zwei Simulationsexperimenten einer modifi- 
zierten Version des GTAP-Modells (Studie Lodefalk und Kinnman 2006). Da regionale simulierte Wohlfahrtsveränderungen stets einem bestimmten Simulationsexperiment entstammen, werden alle Einzelbeobachtungen mit besonders starkem Einfluss auf die Regression (hoher Wert für das Cook's Distance Maß) im Meta-Datensatz belassen; stattdessen werden jeweils die gesamten Simulationsexperimente als „Ausreißer“ behandelt.

Es werden daher zwei Subsamples („mit B+L“ sowie „ohne B+L“) des verwendeten Meta-Datensatzes vorgestellt und zwar sowohl für die Regression über simulierte Änderungen der Weltwohlfahrt als auch für die Regression über simulierte regionale Wohlfahrtsänderungen. Diese Subsamples sind einerseits das endgültige Literatursample mit 110 Studien (Gründe für die Reduktion des Samples von 230 auf 110 Studien sind in Kapitel 3.6 beschrieben) einschließlich aller Simulationsexperimente ("mit B+L") sowie ein kleineres Sample mit 107 Studien (,ohne B+L“), welches die Papiere und Simulationen basierend auf BDS (verantwortlich für insgesamt 408 Observation) sowie 32 weitere Observationen aus zwei spezifischen Simulationsexperimenten einer ansonsten im Sample verbleibenden Studie (Lodefalk und Kinnman 2006) enthält.

Diejenigen Observationen, welche nicht in den „ohne B+L“ Ergebnissen enthalten sind, weisen bis zu viermal größere simulierte Wohlfahrtseffekte als alle übrigen Studien auf und die simulierten Zollreduktionen, gemessen anhand der zuvor beschriebenen Proxys, sind bis zu fünfmal größer. Das BDS-Modell ist gekennzeichnet durch eine spezielle Kombination von Annahmen (steigende Skalenerträge mit fixem Kapitalstock, hohen Armington-Elastizitäten, eigenen Schätzungen über NTB-Daten und exogene Ströme von Foreign Direct Investment FDI). Die zwei Experimente bei Lodefalk und Kinnman (2006) welche ebenfalls im „ohne B+L" Sample fehlen, basieren auf einen Modellierungsansatz mit monopolistischem Wettbewerb, welcher gemäß Hertel, Keeney, Ivanic und Winters (2006, S. 10) das Modell weniger stabil werden lässt.

Tabelle 2 (Weltwohlfahrtsänderung) und Tabelle 5 (regionale Wohlfahrtsänderungen) zeigen deskriptive Statistiken der verwendeten Variablen; die Maximal- , Minimal- und Mittelwerte in Tabelle 2 beziehen sich auf die Summe der jeweiligen Variablen innerhalb eines Simulationsexperiments über alle regionalen Beobachtungen; die entsprechenden Werte in Tabelle 5 repräsentieren die jeweiligen Beobachtungen auf Ebene der Region $(r, e) \quad(r=1,2, \ldots, R$ mit $R=$ Zahl der Regionen im jeweiligen Datensatz; $e=1,2, \ldots, E$ mit $E=$ Zahl der Simulationsexperimente im jeweiligen Subsample).

Tabelle 1 gruppiert die im Datensatz vorkommenden Modellierungskonzepte anhand gängiger Modellspezifikationen. Im Zusammenhang mit Tabelle 1 ist jedoch zu berücksichtigen, dass die Zuordnung bestimmter Modellierungskonzepte zu den Einzelobservationen lediglich eine Orientierung für diejenigen Leser darstellen soll, die mit der modellbasierten Literatur vertraut sind; aus Tabelle 1 
ergibt sich keine eindeutige Klassifikation hinsichtlich bestimmter Modellansätze, welche bestimmten Ergebnissen eindeutig zugeordnet werden könnten.

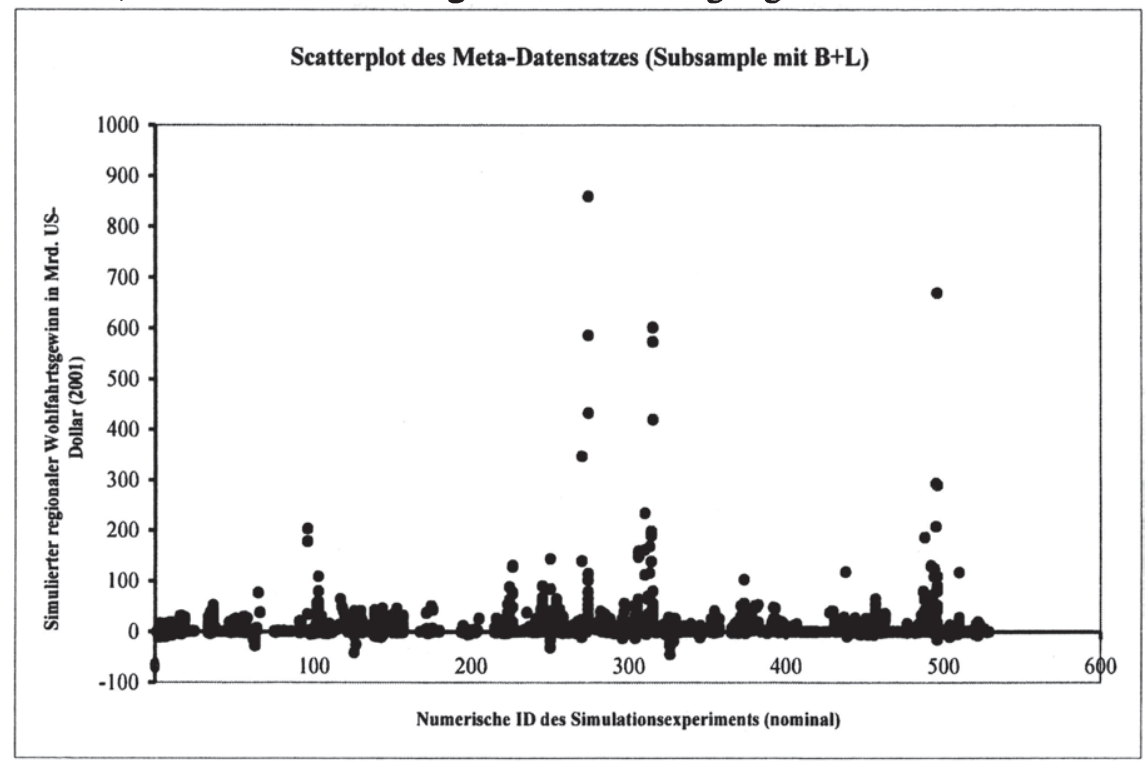

Abbildung 5: Scatterplot regionaler Wohlfahrtsveränderungen je Simulationsexperiment.

Quelle: Eigene Darstellung.

Es ist in diesem Zusammenhang keineswegs eindeutig, worin die Grenzen zwischen zwei Modellierungskonzepten genau bestehen. Hinzu kommt, dass sich verschiedene Modellkonzepte mitunter aus einem gemeinsamen Ursprungsprojekt entwickeln. Gleichzeitig sind die meisten Modellierungskonzepte sehr flexibel, weshalb Modellierer ihre Modelle fur spezifische Fragestellungen in nicht unerheblichem Maße anpassen. Das folgende und das übernächste Kapitel stellen daher Ergebnisse der Meta-Regressionen über Weltwohlfahrtseffekte und regionale Wohlfahrtseffekte vor und erläutern, für welche Modellansätze und Annahmen innerhalb der in Tabelle 1 aufgefuhrten Modellkonzepte ein Einfluss auf simulierte Ergebnisse festgestellt werden kann. 
Tabelle 1: Beschreibung der Modellierungskonzepte und Studien in dem Meta-Datensatz.

\begin{tabular}{|c|c|c|c|c|c|c|}
\hline Modell & $\begin{array}{l}\text { Modell- } \\
\text { ansatz }\end{array}$ & $\begin{array}{c}\text { Annahmen hin- } \\
\text { sichtlich Wett- } \\
\text { bewerb und Ska- } \\
\text { lenertrăge }\end{array}$ & $\begin{array}{l}\text { Komparativ- } \\
\text { statisch oder } \\
\text { dynamisch* }\end{array}$ & $\begin{array}{l}\text { Armington- } \\
\text { Annahme }\end{array}$ & $\begin{array}{c}\text { Anzahl } \\
\text { der Stu- } \\
\text { dien }\end{array}$ & $\begin{array}{l}\text { Beob. } \\
\text { im Da- } \\
\text { ten-satz }\end{array}$ \\
\hline ATPSM & PE & perfekt, CRTS & komp. stat. & nein & 4 & 38 \\
\hline ATPSM & $\mathrm{PE}$ & perfekt, CRTS & komp. stat. & ja & 3 & 495 \\
\hline BDS & GE & imperfekt, IRTS & komp. stat. & ja & 3 & 408 \\
\hline CAPRI & PE & perfekt, CRTS & dynamisch & ja & 1 & 3 \\
\hline CAPSIM & PE & perfekt, CRTS & komp. stat. & nein & 1 & 2 \\
\hline China WTO & $\mathrm{GE}$ & perfekt, CRTS & komp. stat. & $\mathrm{ja}$ & 2 & 52 \\
\hline DRC Council Beijing & $\mathrm{GE}$ & perfekt, CRTS & dynamisch & ja & 1 & 5 \\
\hline Exter & GE & perfekt, CRTS & komp. stat. & $\mathrm{ja}$ & 1 & 4 \\
\hline Francois & GE & perfekt, CRTS & komp. stat. & ja & 1 & 45 \\
\hline Francois & GE & imperfekt, IRTS & komp. stat. & ja & 7 & 462 \\
\hline FSM & PE & perfekt, CRTS & komp. stat. & nein & 1 & 3 \\
\hline FTAP & GE & imperfekt, IRTS & dynamisch & ja & 1 & 18 \\
\hline GROUNDNUT & $\mathrm{PE}$ & perfekt, CRTS & komp. stat. & nein & 3 & 115 \\
\hline GTAP & $\mathrm{GE}$ & perfekt, CRTS & komp. stat. & $\mathrm{ja}$ & 44 & 2340 \\
\hline GTAP & GE & imperfekt, IRTS & komp. stat. & ja & 2 & 99 \\
\hline GTAP-Agr & GE & perfekt, CRTS & komp. stat. & ja & 1 & 51 \\
\hline GTAP-dyn & GE & perfekt, CRTS & dynamisch & ja & 1 & 38 \\
\hline GTEM & GE & perfekt, CRTS & dynamisch & ja & 1 & 48 \\
\hline HRTmr & GE & perfekt, CRTS & komp. stat. & ja & 2 & 222 \\
\hline HRTmr & GE & imperfekt, IRTS & dynamisch & ja & 1 & 15 \\
\hline LINKAGE & GE & perfekt, CRTS & dynamisch & ja & 7 & 450 \\
\hline LINKAGEoecd & $\mathrm{GE}$ & perfekt, CRTS & dynamisch & ja & 1 & 15 \\
\hline LTEM & PE & perfekt, CRTS & dynamisch & nein & 1 & 8 \\
\hline MEGABARE & GE & perfekt, CRTS & komp. stat. & ja & $i$ & 13 \\
\hline MIRAGE & $\mathrm{GE}$ & perfekt, CRTS & komp. stat. & ja & 1 & 187 \\
\hline MIRAGE & GE & perfekt, CRTS & dynamisch & ja & 1 & 24 \\
\hline MIRAGE & $\mathrm{GE}$ & imperfekt, IRTS & komp. stat. & ja & 1 & 5 \\
\hline MIRAGE & GE & imperfekt, IRTS & dynamisch & ja & 1 & 150 \\
\hline MRT-SS & GE & perfekt, CRTS & dynamisch & ja & 1 & 117 \\
\hline ORANImodified & GE & perfekt, CRTS & komp. stat. & nein & $i$ & 1 \\
\hline Hoekman et al. & PE & perfekt, CRTS & komp. stat. & nein & 1 & 242 \\
\hline Tumbarello & PE & perfekt, CRTS & komp. stat. & ja & 1 & 14 \\
\hline Hosoe et al. & GE & perfekt, CRTS & komp. stat. & ja & 3 & 24 \\
\hline Shantong-Zhai et al. & GE & perfekt, CRTS & dynamisch & ja & 3 & 28 \\
\hline PerroniWhalley & GE & perfekt, CRTS & komp. stat. & ja & 1 & 1 \\
\hline PRCGEM & GE & perfekt, CRTS & komp. stat. & ja & 1 & 2 \\
\hline PRCGEM & GE & perfekt, CRTS & dynamisch & ja & 1 & 2 \\
\hline RATSIM & PE & perfekt, CRTS & komp. stat. & ja & $i$ & 4 \\
\hline Rutherford & GE & perfekt, CRTS & dynamisch & ja & 1 & 70 \\
\hline Summe & & & & & 110 & 5835 \\
\hline
\end{tabular}

Bemerkung: * $P E=$ partial equilibrium, partielles Gleichgewicht; $G E=$ General Equilibrium, allgemeines Gleichgewicht. * Als "dynamisch" sind alle dynamischen Spezifikationen außer komparativ-statischen Modellen basierend auf der Baldwin-Closure (was einige Autoren ebenfalls als 'dynamisch'bezeichnen) klassifiziert. Quelle: Eigene Zusammenstellung.

Gleichung (3.14) wird als multivariates Regressionsmodell für beide Subsamples („mit B+L“ sowie „ohne B+L“) geschätzt, und zwar zunächst auf Ebene simulierter Weltwohlfahrtsänderungen. Diese aggregierte Summe regionaler Wohlfahrtseffekte bezieht sich zunächst immer auf die gesamte Weltvolkswirtschaft, da multiregionale CGE-Modelle stets alle Länder mit einschließen. 
In einigen Fällen bilden single country CGEs sowie insbesondere partielle Gleichgewichtsmodelle jedoch nicht die gesamte Weltvolkswirtschaft ab. Dennoch kann auch bei diesen Simulationsexperimenten von „Weltwohlfahrtsänderungen" gesprochen werden, da ein partielles Gleichgewichtsmodell unterstellt, dass die Geschehnisse innerhalb des nicht explizit modellierten Teils der Volkswirtschaft durch die simulierten Politikänderungen unberührt bleiben und Interaktionen der modellierten Sektoren und Regionen mit der übrigen Weltvolkswirtschaft vernachlässigt werden können.

Wie in Kapitel 3.6.1 erläutert, kommen für alle Meta-Regressionen auf Basis von Gleichung (3.14) zwei unterschiedliche Gewichtungsschemata in Frage: Die ungewichtete Regression betrachtet das Literatursample und erklärt anhand der Kovariate die Varianz innerhalb der abhängigen Variable „Weltwohlfahrtsänderung". Die anhand des Schemas [1/Zahl der Observationen aus einer Studie] gewichtete Regression misst die durchschnittliche Höhe des Einflusses einer erklärenden Variable auf die abhängige Variable. Aussagen über die relative und absolute Bedeutung der durch die erklärenden Variablen abgebildeten Modellannahmen sollten anhand dieser gewichteten Regression getroffen werden, da die Koeffizienten der ungewichteten Regression aufgrund unterschiedlich häufig innerhalb des Datensatzes vorkommender Modellansätze (vgl. Tabelle 1) die tatsächliche Wirkungsweise selten vorkommender Modellannahmen, wie beispielsweise der Johansen-Macro-Closure, evtl. verzerrt wiedergeben (vergleiche die Koeffizienten dieser Variable in Tabelle 3 und 4).

Ein Vergleich der Tabellen 3, 4, 6, 7 und 8 zeigt, dass das geschätzte Regressionsmodell (Gleichung 3.14) hinsichtlich der ausgewiesenen Koeffizienten, Signifikanzniveaus und Determinationskoeffizienten eine große Übereinstimmung zwischen den jeweiligen Subsamples und Gewichtungsschemata aufweist. Der Determinationskoeffizient der ungewichteten Regression (Tabelle 3 und Tabelle 4) zeigt, dass 61 Prozent der Variation simulierter Weltwohlfahrtsänderungen des „ohne $B+L$ “ Datensatzes durch die gewählten Variablen erklärt werden (40 Prozent des „mit B+L“ Samples). Bedenkt man die approximative Natur vieler erklärender Variablen innerhalb der Regression, erscheint dieses Ergebnis überraschend hoch. Das $\mathrm{R}^{2}$ in der gewichteten Regression enthält eine transformierte abhängige Variable und kann nicht auf die gleiche Art interpretiert werden.

Die geschätzten Koeffizienten der Weltwohlfahrtsregression (Tabellen 3 und 4) sind dabei ihrem Betrag nach erwartungsgemäß höher als die Koeffizienten für regionale Wohlfahrtseffekte. Aufgrund der hohen Übereinstimmung der Vor-zeichen und Signifikanzniveaus aller Koeffizienten erfolgt eine eingehende Interpretation der ausgewiesenen Effekte in Kapitel 4.3.1 im Zusammenhang mit der Meta-Regression regionaler Wohlfahrtsänderungen. 
Abbildung 6 zeigt die jeweils errechneten predicted values der MetaRegression in Tabelle 4 (gewichtet, Sample „ohne B+L“), d.h. die isoliert liegenden Datenpunkte bezeichnen Werte für Weltwohlfahrtsänderungen, welche im Mittel des Literatursamples von 107 Publikationen für unterschiedliche Modellspezifikationen ausgewiesen werden, sofern man folgendes Politikszenario unterstellt und die entsprechenden Werte in die geschätzte Regressionsgleichung einsetzt: Anhand eines allgemeinen Gleichgewichtsmodells mit einer zu 10 Regionen und 10 Sektoren aggregierten GTAP-6-Datenbank, wobei 8 der 10 Sektoren Agrarprodukte bzw. verarbeitete Lebensmittel darstellen, wird eine vollständige Abschaffung aller angewandten Zölle in allen Sektoren und Regionen simuliert, wobei die Datenbasis vor der eigentlichen Simulation keine Projektion erfährt. Alle übrigen Werte für die erklärenden Variablen in Tabelle 4 nehmen hierbei den Wert Null an.

Die Spezifikationen dieses Zollkürzungsexperiments wurden zudem in die unter Kapitel 3 beschriebene Referenzdatenbank (vgl. Abbildung 4) eingegeben, um für die jeweiligen Variablen Proxywerte zu erhalten, welche mit den geschätzten Koeffizienten der Meta-Regression (Tabelle 4, "ohne B+L“, gewichtet) vergleichbar sind. Anhand dieser Werte wurden mit den in Tabelle 4, Spalte "ohne $\mathrm{B}+\mathrm{L}$ “ ausgewiesenen Koeffizienten predicted values errechnet (vgl. Kapitel 3.2.9) und zwar unter Hinzuziehung der geschätzten Koeffizienten für alternative Modellspezifikationen dynamischer und komparativ-statischer Modelle (Tabelle 4). Für die Länge der Simulationsdauer dynamischer Modelle wurde ein Jahr unterstellt und in die geschätzte Regressionsgleichung eingesetzt, um Vergleiche mit komparativ-statischen Modellen innerhalb von Abbildung 6 zu ermöglichen. Dynamische Modelle würden innerhalb der Literatur deutlich andere absolute Werte aufweisen als die abgebildeten predicted values ausweisen, da sich Simulationszeiträume gewöhnlich über mehrere Perioden erstrecken (vgl. die Diskussion der Koeffizienten für dynamische Modellansätze in Kapitel 4.3.1).

Abbildung 6 zeigt ferner die so ermittelten predicted values der „ohne $\mathrm{B}+\mathrm{L}$ “ Regression in Tabelle 4 im Vergleich mit simulierten Welt-Wohlfahrtseffekten des Standard GTAP-Modells. Hierzu wurde das GTAP-Modell (RunGTAP Version 3.4) in Verbindung mit der GTAP Datenbank 6.01 verwendet, um das oben beschriebene Zollkürzungsszenario (Abschaffung aller angewandten Zölle) zu simulieren. Die GTAP Datenbank wurde auf 10 Länder und 10 Sektoren aggregiert, wobei 8 der 10 Sektoren Agrarprodukte bzw. verarbeitete Lebensmittel darstellen. In den übrigen beiden Sektoren wurden "Services“ sowie „Manufacturing \& Natural Ressources“ zusammengefasst. Die geografische Aggregation der Regionen ist Anhang $C$ zu entnehmen. Anhang $C$ enthält sowohl eine Kopie der .cmf Datei zur Reproduktion der Simulationsergebnisse (enthält u.a. Modell-Closure und Politikschock), als auch die Datei zur Aggregation der 
Datenbank. Dieses Simulationsexperiment wurde mehrfach unter alternativen Spezifikationen der GTAP Armington-Elastizitäten ESUBD und ESUBM wiederholt; die dabei errechneten Weltwohlfahrtsänderungen sind entlang der diagonalen Trendlinie in Abbildung 6 abgetragen. Die Abszisse in Abbildung 6 weist ein aggregiertes $\mathrm{Maß}$ für die Höhe der verwendeten Armington-Elastizitäten aus. Das Standard GTAP Modell spezifiziert für alle Regionen jeweils die gleichen Werte für ESUBD und ESUBM; diese Werte unterscheiden sich jedoch für die aggregierten Sektoren. Bildet man jeweils den Durchschnitt der Standardwerte für ESUBD und ESUBM und addiert diese beiden Durchschnitte, erhält man die in Abbildung 6 abgetragenen Werte der Abszisse. Zur Simulation mit alternativen Armington-Elastizitäten wurden die sektoralen Ausgangswerte für ESUBD und ESUBM schrittweise jeweils um den Wert 2 erhöht.

Da innerhalb des Meta-Datensatzes nur zwischen „niedrigen“ und „hohen“ Armington-Elastizitäten unterschieden werden konnte, müssen die errechneten predicted values der Meta-Regression in Abbildung 6 diesbezüglich den Werten im Standard GTAP Modell zugeordnet werden. Gemäß der Definition im MetaDatensatz für ,niedrige“ Armington-Elastizitäten entspricht dies näherungsweise der Höhe der Ausgangs-Armington-Elastizitäten im Standard GTAP Modell.

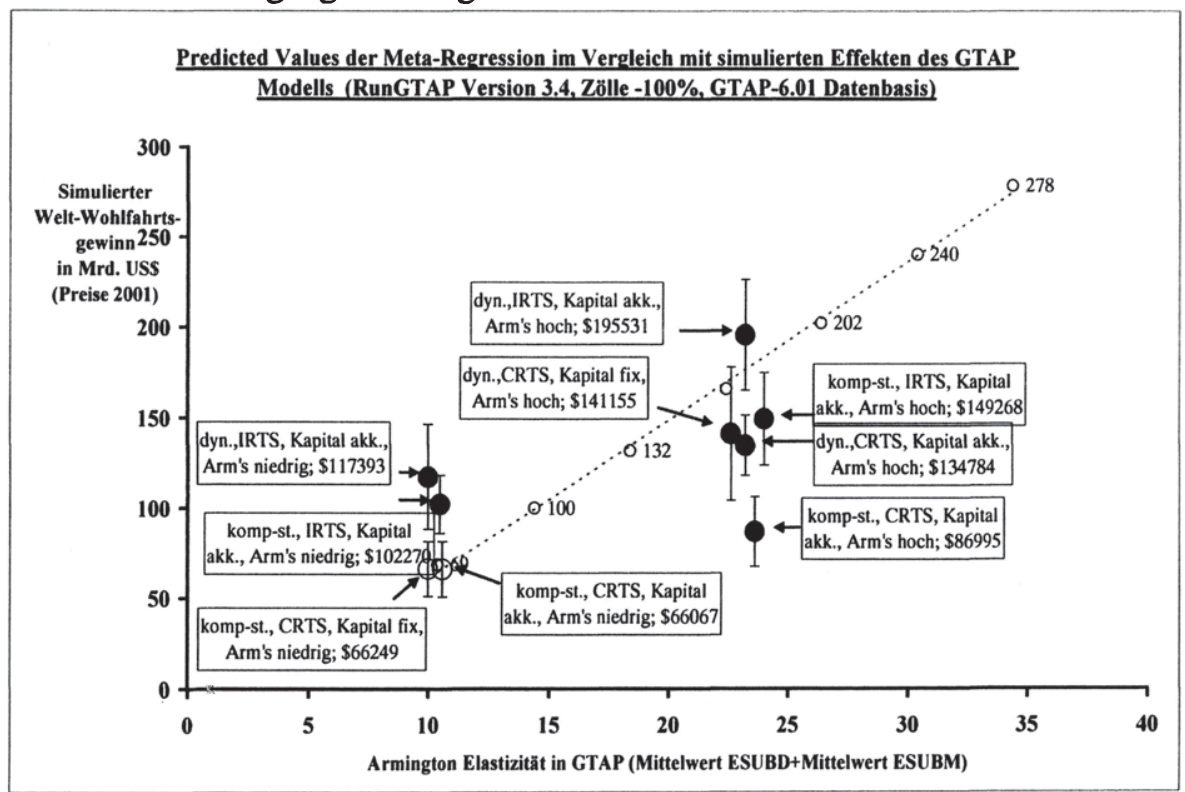

Abbildung 6: Armington-Elastizitäten und weitere Modellspezifikationen im Vergleich.

Erläuterung im Text.

Quelle: Eigene Darstellung. 
Die abgetragenen Durchschnittswerte für „hohe“ Armington-Elastizitäten wurden bei ca. der zweifachen Höhe der Standardwerte zwischen den Armington-Summen 22,5 und 25 mit geringer Variation zwecks verbesserter Übersicht abgetragen (die genaue Position der predicted values innerhalb dieses Intervalls ist dabei willkürlich gewählt). Der tatsächliche Durchschnitt der innerhalb des Literatursamples verwendeten „hohen“ Armington-Elastizitäten ist dabei unbekannt und könnte deutlich höher liegen, da viele Autoren die Höhe ihrer Armington-Elastizitäten nur qualitativ einordnen (,sehr hoch“, „doppelt so hoch wie GTAP“, etc.). Aufgrund der eingeschränkten Vergleichbarkeit dieser Werte im Rahmen einer bivariaten Darstellung muss betont werden, dass durch Abbildung 6 lediglich eine Einschätzung der relativen Größenordnungen geschätzter Koeffizienten im Vergleich zu einer typischen, wenn auch einfachen, Simulation anhand des Standard GTAP Modells erreicht werden soll.

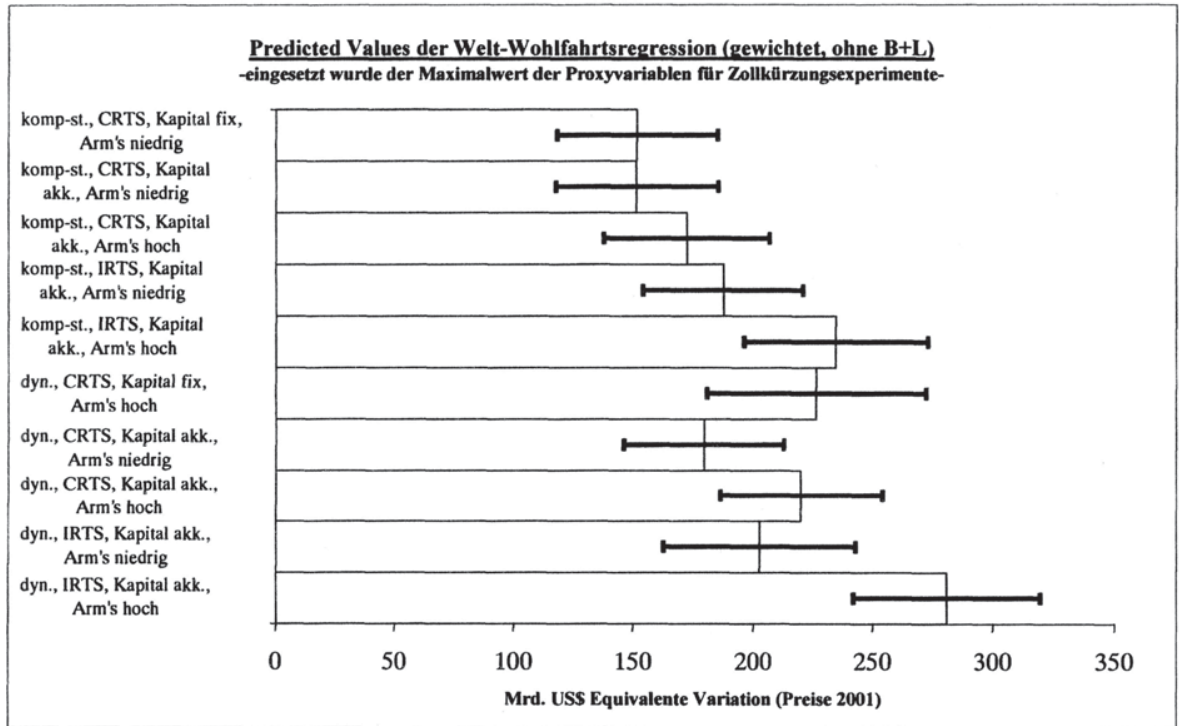

Abbildung 7: Predicted Values der Weltwohlfahrtsregression Subsample „ohne B+L“, gewichtet. Quelle: Eigene Berechnung.

Unter Berücksichtigung dieser Ungenauigkeiten kann aus Abbildung 6 jedoch entnommen werden, dass die Größenordnungen simulierter Wohlfahrtseffekte (68611 Mio. US-Dollar) und geschätzter Werte der Meta-Regression für eine vergleichbare Modellspezifikation sehr ähnliche Größenordnungen aufweisen (predicted value $\hat{Y}=65770$ Mio. US-Dollar). Ferner wird durch eine Verdreifachung der durchschnittlichen Armington-Elastizitäten ESUBD und ESUBM im Standard GTAP Modell bereits eine 
fahrtsänderung errechnet als mit Standardparametern. Diese simulierten Weltwohlfahrtsgewinne mit höheren Armington-Elastizitäten liegen ca. halb so hoch wie der maximale Wohlfahrtsgewinn des „ohne B+L“ Literatursamples (vgl. Tabelle 2), in welchem sehr unterschiedliche Modellansätze, Modellkonzepte und Liberalisierungsszenarien vertreten sind.

Abbildung 7 zeigt predicted values für die gleiche 10×10 Aggregation der GTAP 6 Datenbank (Anhang C), welche auch Abbildung 6 zu Grunde liegt.

Anstelle des dort simulierten Zollkürzungsexperiments wurde jedoch der Maximalwert der Variablen „Änderungen von Zöllen, Exportsubventionen und ,Amber'-Box Maßnahmen“, welcher im „ohne B+L“ Sample (Tabelle 2) ausgewiesen ist, eingesetzt. Abbildung 7 kann also verstanden werden als eine Ausweisung von predicted values unterschiedlicher Modellansätze für das ambitionierteste Liberalisierungsexperiment, welches im Literatursample ausgewiesen ist.

Eine weltweite Abschaffung aller Zölle, Exportsubventionen und AmberBox Maßnahmen würde demzufolge je nach unterstellter Modellspezifikation einen durchschnittlich simulierten Weltwohlfahrtsgewinn zwischen 130000 Mio. US-Dollar und 330000 Mio. US-Dollar hervorbringen. 
Tabelle 2: Deskriptive Statistik der verwendeten Variablen in den Regression für aggregierte Weltwohlfahrtsgewinne.

\begin{tabular}{|c|c|c|c|c|c|c|c|c|c|c|}
\hline \multirow{2}{*}{ Kat } & \multirow{2}{*}{ Variable } & \multirow{2}{*}{ Einheit } & \multicolumn{4}{|c|}{$\begin{array}{l}\text { Stichprobe mit } B+L^{2} \\
\text { (110 Studien, } n=481 \text { ) }\end{array}$} & \multicolumn{4}{|c|}{$\begin{array}{l}\text { Stichprobe ohne } B+L^{2} \\
(107 \text { Studien, } n=459)\end{array}$} \\
\hline & & & Min. & Max. & Mittel & Std. Abw. & Min. & Max. & Mittel & $\begin{array}{r}\text { Std. } \\
\text { Abw. }\end{array}$ \\
\hline $\mathbf{Y}$ & Simulierte Wohlfahrtsänderung & Mio. US\$ & -87370 & 2587000 & 60740 & 188807 & -87370 & 539800 & 40810 & 71617 \\
\hline M & Abhängige Variable: prozentuale Änderung des GDP & Dummy & 0 & 1 & 0,089 & - & 0 & 1 & 0,094 & - \\
\hline $\mathbf{M}$ & Abhängige Variable: absolute Änderung des GDP & Dummy & 0 & 1 & 0,116 & - & 0 & 1 & 0,122 & - \\
\hline $\mathbf{M}$ & Abhängige Variable: PE Wohlfahrt (Dummy für $P E=1$ ) & Dummy & $\mathbf{0}$ & 1 & 0,091 & $\cdot$ & 0 & 1 & 0,096 & - \\
\hline $\mathbf{M}$ & Partielles Gleichgewichtsmodell (Dummy für $\mathrm{PE}=1$ ) & Dummy & $\mathbf{0}$ & 1 & 0,116 & - & 0 & 1 & 0,122 & - \\
\hline $\mathbf{M}$ & Modell mit Johansen-Macro-Closure (Dummy für ja=1) & Dummy & $\mathbf{0}$ & 1 & 0,064 & - & 0 & 1 & 0,024 & - \\
\hline $\mathbf{M}$ & Dynam., CRTS, perf. Wettb., fixer Kapitalst., hohe Arm.-El. & Dummy & 0 & 1 & 0,006 & - & 0 & 1 & 0,007 & - \\
\hline $\mathbf{M}$ & Dynam., CRTS, perf. Wettb., fixer Kapitalst., niedrige Arm.-El. & Dummy & 0 & 1 & 0,150 & - & 0 & 1 & 0,157 & - \\
\hline M & Dynam., CRTS, perf. Wettb., steig. Kapitalst., hohe Arm.-El. & Dummy & $\mathbf{0}$ & 1 & 0,046 & - & 0 & 1 & 0,048 & - \\
\hline $\mathbf{M}$ & Dynam., IRTS, imperf. Wettb., steig. Kapitalst., niedr. Arm.-El. & Dummy & 0 & 1 & 0,021 & - & 0 & 1 & 0,022 & - \\
\hline M & Dynam., IRTS, imperf. Wettb., steig. Kapitalst., hohe Arm.-El. & Dummy & 0 & 1 & 0,002 & - & 0 & 1 & 0,002 & - \\
\hline $\mathbf{M}$ & Komp. stat., CRTS, perf. Wettb., steig. Kapitalst., niedr. Arm.-El. & Dummy & 0 & 1 & 0,181 & - & 0 & 1 & 0,190 & - \\
\hline M & Komp. stat., CRTS, perf. Wettb., steig. Kapitalst., hohe Arm.-El. & Dummy & 0 & 1 & 0,035 & - & 0 & 1 & 0,037 & - \\
\hline M & Komp. stat., IRTS, imperf. Wettb., fixer Kapitalst., hohe Arm.-El. & Dummy & 0 & 1 & 0,042 & - & 0 & - & - & - \\
\hline M & Komp. stat., IRTS, imperf. Wettb., steig. Kap., niedrige Arm.-El. & Dummy & 0 & 1 & 0,075 & - & 0 & 1 & 0,078 & - \\
\hline$M$ & Komp. stat., IRTS, imperf. Wettb., steig. Kapitalst., hohe Arm.-E. & Dummy & 0 & 1 & 0,019 & - & 0 & 1 & 0,015 & - \\
\hline 章: & Zusätzliche Beschränkungen von Faktormobilität/-angebot & Dummy & 0 & 1 & 0,173 & - & 0 & 1 & 0,181 & - \\
\hline $\mathbf{M}$ & Pre-simulative Datenprojektion: Zeitraum in Jahren & Jahre & $\mathbf{0}$ & 28 & 5,435 & 3,35 & 0 & 28 & 5,294 & 3,320 \\
\hline M & Dynamische Modelle: Lảnge der Simulationsdauer in & Jahre & 0 & 14 & 1,462 & 7,03 & 0 & 14 & 1,471 & 7,140 \\
\hline No & Handelsbilanz eines oder mehrerer Länder ist fix & Dummy & $\mathbf{0}$ & 1 & 0,179 & - & 0 & 1 & 0,155 & \\
\hline $\mathbf{M}$ & Anzahl der Regionen & Anzahl & 1 & 21,54 & 161,0 & 31,79 & 1 & 161 & 21,59 & 32,54 \\
\hline M & Anzahl der Sektoren & Anzahl & 1 & 18,30 & 78,00 & 11,88 & 1 & 78 & 18,02 & 12,16 \\
\hline$M^{0}$ & Anzahl der aggregierten Agrarprodukte & Anzahl & 3 & 158 & 9,116 & 12,91 & 1 & 158 & 9,553 & 13,06 \\
\hline M & Autor erwähnt ad hoc Modifikationen verwendeter Elastizitäten & Dummy & 0 & 1 & 0,021 & - & 0 & 1 & 0,022 & 年 \\
\hline Mu & Autor benutzt Elastizităten aus eigenen ökonometr. Schätzungen & Dummy & 0 & 1 & 0,023 & - & 0 & 1 & 0,024 & - \\
\hline $\overrightarrow{P_{1}}$ & Änderung von Zöllen, Exp.-Subv. und Amber-Box Maßnahmen & Mio. US\$ & -40160000 & 936100 & -766600 & 2641900 & -5969000 & 202200 & -586700 & 1010210 \\
\hline P & Änderungen von Non-Tariff-Barriers & Mio. US\$ & -9252000 & 0 & -188500 & 1037876 & -9252000 & 0 & -180100 & 1030532 \\
\hline $\mathrm{PO}$ & Änderungen von Blue/Green-Box Maßnah & Mio. US\$ & -14090000 & 766700 & -612600 & 1836024 & -14090000 & 766700 & -593500 & 1805052 \\
\hline $\overrightarrow{\mathbf{R}^{1}}$ & Simulierte Änderung von technischem Fortschritt o.Ä. & Mio. US\$ & -618500 & 15450 & -2125 & 32413 & -618500 & 15450 & -2227 & 33179 \\
\hline D & Datenbasis GTAP-4 & Dummy & 0 & 1 & 0,140 & - & 1 & 0 & 0,129 & 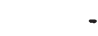 \\
\hline D & Datenbasis GTAP-5 & Dummy & 0 & 1 & 0,330 & - & 1 & 0 & 0,331 & - \\
\hline D & Datenbasis GTAP-6 & Dummy & 0 & 1 & 0,160 & - & 1 & 0 & 0,166 & - \\
\hline D & Nicht-GTAP Datenbasis & Dummy & 0 & 1 & 0,210 & - & 1 & 0 & 0,227 & \\
\hline
\end{tabular}


Tabelle 3: OLS-Regression über simulierte Weltwohlfahrtsänderungen (ungewichtet, robuste Standardfehler).

\begin{tabular}{|c|c|c|c|c|c|c|c|c|c|c|}
\hline \multirow{2}{*}{$\begin{array}{r}\text { Gewichtungsschema } \rightarrow \\
\text { Stichprobe } \rightarrow \\
\end{array}$} & \multicolumn{10}{|c|}{ Ungewichtet } \\
\hline & \multicolumn{5}{|c|}{ mit $B+L^{2}$} & \multicolumn{5}{|c|}{ ohne $B+L^{2}$} \\
\hline IVariable & Koeff. & Std. Fehl. & t-Wert & $\operatorname{Pr}(>t)$ & & Koeff. & Std. Fehl. & t-Wert & $\operatorname{Pr}(>t \mid)$ & \\
\hline Achsenabschnitt & 9572,7 & 10411,2 & 0,9195 & 0,3584 & & 18595,4 & 6926,0 & 2,6849 & 0,0075 & \#** \\
\hline Abhängige Variable: prozentuale Änderung des GDP & 19732,0 & 12588,5 & 1,5675 & 0,1177 & & 16463,7 & 10072,0 & 1,6346 & 0,1029 & \\
\hline Abhängige Variable: absolute Änderung des GDP in Mio. US\$ & 23216,1 & 11981,2 & 1,9377 & 0,0533 & - & 23190,0 & 11117,7 & 2,0859 & 0,0376 & $* *$ \\
\hline Abhängige Variable: PE Wohlfahrtseffekte (Summe partieller Renten) & 10681,5 & 14368,2 & 0,7434 & 0,4576 & & 6119,1 & 9927,2 & 0,6164 & 0,5380 & \\
\hline Modell: Partielles vs. allgemeines Gleichgewicht $(\mathrm{PE}=1)$ & $-9871,4$ & 25838,9 & $-0,3820$ & 0,7026 & & 9360,6 & 10687,0 & 0,8759 & 0,3816 & \\
\hline Modell verwendet Johansen-Macro-Closure (Dummy für ja=1) & 78283,1 & 41141,8 & 1,9028 & 0,0577 & * & 49787,6 & 23585,1 & 2,1110 & 0,0354 & $* *$ \\
\hline Dyn., CRTS, vollk. Wettb., fixer Kapitalst., hohe Arm.-El. & 139013,7 & 63454,9 & 2,1908 & 0,0290 & ** & 128182,4 & 50637,2 & 2,5314 & 0,0117 & $* *$ \\
\hline Dyn., CRTS, vollk. Wettb., steig. Kapitalst., niedrige Arm.-El. & 19590,7 & 14830,5 & 1,3210 & 0,1872 & & 25740,9 & 9908,0 & 2,5980 & 0,0097 & *** \\
\hline Dyn., CRTS, vollk. Wettb., steig. Kapitalst., hohe Arm.-El. & 76457,5 & 17183,9 & 4,4494 & 0,0000 & *** & 79112,8 & 12161,6 & 6,5052 & 0,0000 & *** \\
\hline Dyn., IRTS, unvollk. Wettb., steig. Kapitalst., niedrige Arm.-El. & 67965,7 & 23492,0 & 2,8931 & 0,0040 & $* * *$ & 63741,8 & 17367,6 & 3,6702 & 0,0003 & *** \\
\hline Byn., IRTS, unvollk. Wettb, steig. Kapitalst., hohe Arm.-El. & 73224,7 & 71442,2 & 1,0250 & 0,3059 & & 142658,9 & 24056,2 & 5,9302 & 0,0000 & $* *$ \\
\hline Komp. stat., CRTS, vollk. Wettb., steig. Kapitalst., niedrige Arm.-El. & 6572,0 & 10704,9 & 0,6139 & 0,5396 & & 694,7 & 6427,2 & 0,1081 & 0,9140 & \\
\hline Komp. stat., CRTS, vollk. Wettb., steig. Kapitalst., hohe Arm.-El. & 33633,7 & 18197,2 & 1,8483 & 0,0652 & * & 36908,3 & 12261,1 & 3,0102 & 0,0028 & $* *$ \\
\hline ISomp. stat., IRTS, unvollk. Wettb., fixer Kapitalst., hohe Arm.-El. & 290089,2 & 147131,9 & 1,9716 & 0,0493 & ** & & & - & & \\
\hline Komp. stat., IRTS, unvollk. Wettb., steig. Kapitalst., niedrige Arm.-El. & 8016,3 & 13136,3 & 0,6102 & 0,5420 & & 15527,6 & 11151,4 & 1,3924 & 0,1645 & \\
\hline Komp. stat., IRTS, unvollk. Wettb., steig. Kapitalst., hohe Arm.-EI. & 29596,5 & 53880,0 & 0,5493 & 0,5831 & & 78761,5 & 40691,9 & 1,9356 & 0,0536 & * \\
\hline Zusätzliche Beschränkungen von Faktormobilität/-angebot & $-5617,8$ & 13705,4 & $-0,4099$ & 0,6821 & & 1570,1 & 7795,8 & 0,2014 & 0,8405 & \\
\hline Pre-simulative Datenprojektion: Zeitraum in Jahren & $-3235,2$ & 1268,7 & $-2,5500$ & 0,0111 & $*$ & $-3313,5$ & 824,4 & $-4,0194$ & 0,0001 & **** \\
\hline Dynamische Modelle: Länge der Simulationsdauer in Jahren & $-4247,7$ & 1398,6 & $-3,0371$ & 0,0025 & $* * *$ & $-3896,4$ & 990,2 & $-3,9348$ & 0,0001 & *** \\
\hline [Pypamische Modelle: Länge der Simulationsdauer in Jahren]^2 & 194,4 & 70,4 & 2,7599 & 0,0060 & *** & 141,4 & 44,6 & 3,1700 & 0,0016 & *** \\
\hline Bandelsbilanz eines oder mehrerer Länder ist fix & $-35787,9$ & 42809,1 & $-0,8360$ & 0,4036 & & 1886,0 & 6406,3 & 0,2944 & 0,7686 & \\
\hline Anzahl der Regionen & $-4,9$ & 99,5 & $-0,0489$ & 0,9610 & & 72,1 & 59,9 & 1,2029 & 0,2297 & \\
\hline Anzăl der Sektoren & 24,6 & 329,9 & 0,0747 & 0,9405 & & 29,2 & 227,6 & 0,1283 & 0,8980 & \\
\hline Anzanil der aggregierten Agrarprodukte & $-538,1$ & 244,0 & $-2,2057$ & 0,0279 & $* * *$ & $-597,3$ & 201,1 & $-2,9698$ & 0,0031 & *** \\
\hline Regiọnen "Sektoren & $-3,7$ & 18,4 & $-0,1988$ & 0,8425 & & $-6,4$ & 11,6 & $-0,5489$ & 0,5834 & \\
\hline Aùtóf erwähnt ad hoc Modifikationen verwendeter Elastizitāten & $-33492,6$ & 29153,3 & $-1,1488$ & 0,2512 & & $-40265,4$ & 13508,0 & $-2,9809$ & 0,0030 & *** \\
\hline Autoó benutzt Elastizitäten aus eigenen ökonometr. Schätzungen & 15510,7 & 9639,7 & 1,6090 & 0,1083 & & 11764,1 & 6631,1 & 1,7741 & 0,0768 & - \\
\hline Anderung von Zöllen, Exp.-Subv. und Amber-Box MaBnahmen & $-0,0696$ & 0,0252 & $-2,7633$ & 0,0060 & $* * *$ & $-0,0327$ & 0,0113 & $-2,8920$ & 0,0040 & $* * *$ \\
\hline [Anderung von Zöllen, Exp.-Subv. und Amber-Box Maßnahmen]^ 2 & 0,0000 & 0,0000 & $-1,5710$ & 0,1169 & & 0,0000 & 0,0000 & 0,5669 & 0,5711 & \\
\hline Ânderzungen von Non-Tariff-Barriers & $-0,0112$ & 0,0085 & $-1,3130$ & 0,1899 & & $-0,0170$ & 0,0059 & $-2,8805$ & 0,0042 & *** \\
\hline ÂdGrungen von Blue/Green-Box MaBnahmen & 0,0116 & 0,0070 & 1,6434 & 0,1010 & & 0,0029 & 0,0018 & 1,6120 & 0,1077 & \\
\hline Simullierte Änderung von technischem Fortschritt o.Ä. & 0,1709 & 0,0258 & 6,6226 & 0,0000 & $* * *$ & 0,1902 & 0,0198 & 9,5988 & 0,0000 & *** \\
\hline Dateribasis GTAP-4 & 16529,9 & 27954,0 & 0,5913 & 0,5546 & & 20230,7 & 13032,8 & 1,5523 & 0,1213 & \\
\hline Batenibasis GTAP-5 & 5362,8 & 14859,2 & 0,3609 & 0,7183 & & 5092,3 & 7440,2 & 0,6844 & 0,4941 & \\
\hline E)tentbasis GTAP-6 & $-28911,8$ & 17334,5 & $-1,6679$ & 0,0960 & * & $-13450,9$ & 9683,3 & $-1,3891$ & 0,1655 & \\
\hline Nicht-GTAP Datenbasis & 13060,2 & 36421,6 & 0,3586 & 0,7201 & & $-18344,3$ & 12944,7 & $-1,4171$ & 0,1572 & \\
\hline Residual Standard Error / Freil & & 14620 & $445 / 0,40$ & & & & 44500 & $424 / 0,6$ & & \\
\hline
\end{tabular}


Tabelle 4: OLS Regression über simulierte Weltwohlfahrtsänderungen (gewichtet, robuste Standardfehler).

\begin{tabular}{|c|c|c|c|c|c|c|c|c|c|c|}
\hline Gewichtungsschema $\rightarrow$ & \multicolumn{10}{|c|}{ Gewichtet mit [1/(Beobachtungen in der Studie)] } \\
\hline Stichprobe $\rightarrow$ & \multicolumn{5}{|c|}{ mit $B+L^{a}$} & \multicolumn{5}{|c|}{ ohne $B+L^{a}$} \\
\hline IVariable & Koeff. & Std. Fehl. & $t$-Wert & $\operatorname{Pr}(>\mid t)$ & & Koeff. & Std. Fehl. & t-Wert & $\operatorname{Pr}(>|t|)$ & \\
\hline Achsenabschnitt & 19827,7 & 1274,9 & 15,5520 & 0,0000 & *** & 22202,5 & 1803,2 & 12,3131 & 0,0000 & *** \\
\hline Abhängige Variable: prozentuale Änderung des GDP & 23440,7 & 1815,5 & 12,9114 & 0,0000 & $* * *$ & 25044,4 & 2998,5 & 8,3524 & 0,0000 & *** \\
\hline Abhängige Variable: absolute Änderung des GDP in Mio. US\$ & 10955,8 & 3064,3 & 3,5753 & 0,0004 & *** & 13948,4 & 6270,1 & 2,2246 & 0,0266 & ** \\
\hline Abhängige Variable: PE Wohlfahrtseffekte (Summe partieller Renten) & 7246,9 & 3634,3 & 1,9940 & 0,0468 & ** & 6135,7 & 3565,9 & 1,7207 & 0,0860 & * \\
\hline Modell: Partielles vs. allgemeines Gleichgewicht $(\mathrm{PE}=1)$ & 740,6 & 2388,9 & 0,3100 & 0,7567 & & 6978,5 & 3565,1 & 1,9575 & 0,0509 & * \\
\hline Modell verwendet Johansen-Macro-Closure (1=ja) & 25960,3 & 3455,3 & 7,5132 & 0,0000 & *** & 22820,4 & 5621,8 & 4,0593 & 0,0001 & *** \\
\hline Dyn., CRTS, vollk. Wettb., fixer Kapitalst, hohe Arm.-El. & 53244,4 & 34841,8 & 1,5282 & 0,1272 & & 76756,4 & 73139,2 & 1,0495 & 0,2946 & \\
\hline Dyt, CRTS, vollk. Wettb., steig. Kapitalst., niedrige Arm.-El. & 26068,5 & 3694,6 & 7,0558 & 0,0000 & *** & 29905,8 & 5361,2 & 5,5781 & 0,0000 & *** \\
\hline Dys్, CRTS, vollk. Wettb., steig. Kapitalst., hohe Arm.-El. & 70984,5 & 7117,3 & 9,9735 & 0,0000 & **** & 70385,1 & 8636,8 & 8,1495 & 0,0000 & *** \\
\hline DyE, IRTS, unvollk. Wettb., steig. Kapitalst., niedrige Arm.-El. & 33156,9 & 25071,4 & 1,3225 & 0,1867 & & 52994,0 & 41179,8 & 1,2869 & 0,1988 & \\
\hline Dy: IRTS, unvollk. Wettb, steig. Kapitalst., hohe Arm.-El. & 72694,8 & 28355,7 & 2,5637 & 0,0107 & ** & 131132,9 & 20687,3 & 6,3388 & 0,0000 & *** \\
\hline Komp. stat., CRTS, vollk. Wettb., steig. Kapitalst., niedrige Arm.-El. & 2028,6 & 3232,5 & 0,6276 & 0,5306 & & $-183,0$ & 2055,5 & $-0,0890$ & 0,9291 & \\
\hline Komp. stat., CRTS, vollk. Wettb., steig. Kapitalst., hohe Arm.-El. & 6867,6 & 7081,9 & 0,9697 & 0,3327 & & 20745,0 & 13547,7 & 1,5313 & 0,1265 & \\
\hline Kō̄p. stat., IRTS, unvollk. Wettb., fixer Kapitalst., hohe Arm.-El. & 336262,4 & 163339,7 & 2,0587 & 0,0401 & ** & & - & - & & \\
\hline Komp. stat., IRTS, unvollk. Wettb., steig. Kapitalst., niedrige Arm.-El. & 31779,0 & 7287,1 & 4,3610 & 0,0000 & *** & 36020,5 & 7283,5 & 4,9455 & 0,0000 & $* * *$ \\
\hline Komp. stat., IRTS, unvollk. Wettb., steig. Kapitalst., hohe Arm.-El. & 68251,6 & 25434,8 & 2,6834 & 0,0076 & $* * *$ & 83018,6 & 50128,0 & 1,6561 & 0,0984 & * \\
\hline Zusitzfaçche Beschränkungen von Faktormobilität/-angebot & $-562,8$ & 2275,9 & $-0,2473$ & 0,8048 & & $-479,7$ & 2797,0 & $-0,1715$ & 0,8639 & \\
\hline Pre-Singulative Datenprojektion: Zeitraum in Jahren & $-4110,3$ & 652,0 & $-6,3038$ & 0,0000 & *** & $-4136,7$ & 1018,6 & $-4,0611$ & 0,0001 & *** \\
\hline Dyạamische Modelle: Länge der Simulationsdauer in Jahren & $-1453,5$ & 495,0 & $-2,9362$ & 0,0035 & $* * *$ & $-1917,6$ & 530,9 & $-3,6116$ & 0,0003 & $* * *$ \\
\hline [Dquamische Modelle: Länge der Simulationsdauer in Jahren]^2 & 59,5 & 16,2 & 3,6670 & 0,0003 & *** & 67,0 & 19,6 & 3,4131 & 0,0007 & *** \\
\hline Handelşbilanz eines oder mehrerer Länder ist fix & $-12374,6$ & 4779,3 & $-2,5892$ & 0,0099 & $* * *$ & $-9103,8$ & 2024,2 & $-4,4974$ & 0,0000 & *** \\
\hline Anzahidder Regionen & 3,7 & 12,8 & 0,2870 & 0,7743 & & 29,6 & 23,5 & 1,2577 & 0,2092 & \\
\hline Anzahíger Sektoren & $-98,7$ & 70,3 & $-1,4032$ & 0,1613 & & $-133,4$ & 80,9 & $-1,6491$ & 0,0999 & * \\
\hline Anzahl ider aggregierten Agrarprodukte & $-185,8$ & 112,3 & $-1,6544$ & 0,0988 & * & $-312,7$ & 114,5 & $-2,7308$ & 0,0066 & $* * *$ \\
\hline Regponen *Sektoren & $-13,1$ & 10,6 & $-1,2326$ & 0,2184 & & 0,4 & 9,5 & 0,0451 & 0,9641 & \\
\hline Autor ęouahnt ad hoc Modi & $-16767,5$ & 9453,4 & $-1,7737$ & 0,0768 & * & $-16130,2$ & 8131,5 & $-1,9837$ & 0,0479 & *** \\
\hline Auter kènutzt Elastizitäten aus eigenen ökonometr. Schätzungen. & 9809,0 & 3592,0 & 2,7308 & 0,0066 & ** & 7510,1 & 3314,7 & 2,2657 & 0,0240 & ** \\
\hline Anderugig von Zöllen, Exp.-Subv. und Amber-Box MaBnahmen & $-0,0707$ & 0,0091 & $-7,7967$ & 0,0000 & $* * *$ & $-0,0270$ & 0,0098 & $-2,7590$ & 0,0060 & $* * *$ \\
\hline ФÄnderüng von Zöllen, Exp.-Subv. und Amber-Box MaBnahmen]^2 & 0,0000 & 0,0000 & $-2,5481$ & 0,0112 & ** & 0,0000 & 0,0000 & 3,0941 & 0,0021 & $* * *$ \\
\hline Anderungen von Non-Tariff-Barriers & $-0,0106$ & 0,0044 & $-2,3921$ & 0,0172 & ** & $-0,0125$ & 0,0068 & $-1,8341$ & 0,0673 & $*$ \\
\hline Anderumgen von Blue/Green-Box MaBnahmen & 0,0054 & 0,0032 & 1,6906 & 0,0916 & * & 0,0028 & 0,0023 & 1,2345 & 0,2177 & \\
\hline Simaliente Änderung von technischem Fortschritt o.Ä. & 0,1721 & 0,0101 & 17,0125 & 0,0000 & $* *$ & 0,1862 & 0,0154 & 12,1244 & 0,0000 & $* * *$ \\
\hline Datenbasis GTAP-4 & $-9695,3$ & 7582,2 & $-1,2787$ & 0,2017 & & $-10655,7$ & 5952,3 & $-1,7902$ & 0,0741 & $*$ \\
\hline Datenbasis GTAP-5 & $-7404,7$ & 4439,0 & $-1,6681$ & 0,0960 & * & $-2511,9$ & 3455,1 & $-0,7270$ & 0,4676 & \\
\hline Datenbasis GTAP-6 & $-27654,1$ & 4158,5 & $-6,6501$ & 0,0000 & $* * *$ & $-20884,4$ & 5600,5 & $-3,7290$ & 0,0002 & $* * *$ \\
\hline Nicht-GTAP Datenbasis & $-22371,4$ & 3356,3 & $-6,6656$ & 0,0000 & $* * *$ & $-24056,1$ & 3672,1 & $-6,5510$ & 0,0000 & $* * *$ \\
\hline Residual Standard Error / Freiheitsgrade / Korrigiertes R2 & \multicolumn{5}{|c|}{$13600 / 445 / 0,5$} & \multicolumn{5}{|c|}{$6673 / 424 / 0,8038$} \\
\hline
\end{tabular}
$13600 / 445 / 0,582$ 


\subsection{Meta-Regression simulierter regionaler Wohlfahrts- änderungen}

Die Tabellen 6, 7 und 9 präsentieren geschätzte Koeffzienten der Meta-Regression für simulierte regionale Wohlfahrtsänderungen. Tabelle 6 enthält dabei die Variable „Handelsvolumen eines Landes als Summe der Sektoren im Modell“". Die Regressionsschätzungen in Tabelle 7 und 8 enthalten statt dieser Variablen für jede aggregierte geographische Region einen Fixed Effect („DummyVariable"). Koeffizienten, Standardfehler und t-Werte für diese geographischen Fixed Effects sind in Anhang B (dort Tabelle 14 und Tabelle 15) für diese ungewichteten und gewichteten Re-gressionen in Tabelle 7 und Tabelle 8 ausgewiesen.

Im Falle regionaler Wohlfahrtsänderungen bezieht sich eine einzelne Observation „Wohlfahrtsänderung in Region $r$ “ nicht, wie im Falle der Weltwohlfahrtsregression im vorangegangenen Kapitel, auf eine vergleichbare Ausgangsgröße (,Wohlfahrtsänderung der Weltvolkswirtschaft in Mio. US-Dollar"). Vielmehr kann erwartet werden, dass größere Sektoren in größeren Ländern ceteris paribus auch größere Wohlfahrtseffekte ausweisen als kleinere Länder. Um für diese Unterschiede $\mathrm{zu}$ kontrollieren, wird in Tabelle 6 das Handelsvolumen eines Landes verwendet; die Regressionen in Tabelle 7 und 8 benötigen hierfür mehrere hundert Dummy-Variablen (siehe Anhang B mit den dort aufgefuhrten Tabellen 14 und 15).

Wie man sehen kann, reduzieren die 440 ,mit B+L“ Observationen den Determinationskoeffizienten $\left(R^{2}\right)$ der Regressionen in Tabelle 6 sowie Tabelle 7 und Tabelle 8 beträchtlich. Dieser Effekt fällt in der gewichteten Regression durchweg geringer aus, da die Gewichtung der ,mit B+L“ Observation von 7,5 Prozent auf ca. 2,7 Prozent reduziert wird, was deutlich macht dass die „B+L“ Observationen viele „Ausreißer“ beinhalten.

Unabhängig vom Gewichtungsschema und dem Ausschluss der „B+L Ausreißer" sind die Ergebnisse in den Tabellen 6, 7 und 8 robust. In den gewichteten Regressionen mit $\mathrm{B}+\mathrm{L}$ sowie ohne $\mathrm{B}+\mathrm{L}$ sind fast alle Koeffizienten signifikant und weisen die erwarteten Vorzeichen in plausiblen Größenordnung auf.

Die Vorzeichen, Signifikanzniveaus und Größenordnungen der Regressionskoeffizienten in den ungewichteten Regressionen stimmen weitgehend mit denjenigen in den gewichteten Regressionen überein, jedoch sind in der ungewichteten Regression weitaus weniger Koeffizienten signifikant oder die Signifikanzniveaus sind niedriger. Gleichzeitig erscheinen Ergebnisse „mit $\mathrm{B}+\mathrm{L}$ “ $\mathrm{Ob}$ servationen weniger homogen hinsichtlich Vorzeichen, Größenordnung und Signifikanz zu sein. Diese Vergleiche bestätigen, dass die B+L Observationen einen 
starken Einfluss auf die Schätzung von Gleichung 3.14 haben und dass die Gewichtungsschemata diesen Einfluss reduzieren.

\subsection{Interpretation der Regressionskoeffizienten}

Im Folgenden werden geschätzte Koeffizienten für individuelle erklärende Variablen diskutiert und dabei wird - sofern nicht anderes erwähnt - Bezug genommen auf die Koeffizienten der gewichteten Regression „ohne B+L“ in Tabelle 6. Hinter den Namen der Variablen ist außerdem in Klammern die jeweilige Kategorie (M, P oder D), welcher die betreffende Variable in Gleichung 3.14 zugeordnet ist, angegeben.

Simulierte Wohlfahrtsgewinne sind signifikant höher für Länder mit größeren Handelsvolumina (P), wobei pro 1 Million US-Dollar Handelsvolumen ca. 9000 US-Dollar höhere Wohlfahrtsgewinne zu verzeichnen sind. Im Falle der Regressionen in Tabellen 7 und 8 ist alternativ eine geographische Region aus Anhang B (bzw. den dort aufgeführten Tabellen 14 und 15) zu wählen und die Höhe des geschätzten Koeffizienten abzulesen. Im Falle der Weltwohlfahrtsregression in den Tabellen 3 und 4 entfällt die Variable für das Handelsvolumen, da sie für alle Beobachtungen näherungsweise gleich ist.

Ergebnisse für die Variable Änderung des Zollsatzes (P) zeigen, dass die Relation zwischen der simulierten Reduktion und dem Wohlfahrtsgewinn nicht linear ist. Kalkulationen mit den Koeffizienten in Tabelle 6 zeigen, dass die simulierten Wohlfahrtsänderungen sich innerhalb des relevanten Bereichs von Zollreduzierungen von 2,3 Milliarden US-Dollar simuliertem Wohlfahrtsverlust bis zu 200 Milliarden US-Dollar simuliertem Wohlfahrtsgewinn erstrecken. Innerhalb dieses Bereichs ändern sich Wohlfahrtsgewinne stärker als die simulierten Zollreduktionen, was durch die Tatsache erklärt wird, dass Wohlfahrtsverluste eine quadratische Funktion der angewandten Zollhöhe sind (von acht Regressionen in den Tabellen 6, 7 und 8 ist der quadratische Term der Zolländerungen in sechs Regressionen signifikant; in Tabelle 3 und Tabelle 4 gilt dies für zwei von vier Regressionen).

Die Ergebnisse der „B+L“ Observationen sind in diesem Zusammenhang nicht auf Anhieb verständlich. Wie oben erwähnt, zeigen die „B+L“ Observationen einen wesentlich größeren Bereich simulierter Zollreduktion an (bis zu 12,79 Milliarden US-Dollar). Innerhalb dieses Bereichs steigen die simulierten Wohlfahrtsgewinne zunächst an und fallen dann oberhalb eines Wertes von 7,5 Milliarden US-Dollar Produktionswert wieder ab. Für Zollreduktionen zwischen 0 und 1,5 Milliarden US-Dollar (gemessen anhand der zuvor beschrieben Proxyvariable), dem Bereich, in welchem die meisten Observationen in beiden 
Samples liegen, sind die Wohlfahrtsgewinne in einem ähnlichen Bereich angesiedelt.

Simulierte Reduktionen der NTBs (P) haben ebenfalls einen negativen Einfluss, aber diese sind niedriger bzw. weniger signifikant, was durch die sehr ungenaue Proxy-Variable erklärt werden kann, durch welche die NTBs quantifiziert wurden (siehe Kapitel 3).

Steigende Ausgaben für ,Blue 6 und ,Green' Box (P) Politiken lassen insgesamt einen moderaten Wohlfahrtsgewinn von 5000 US-Dollar pro 1 Million Dollar Ausgabenanstieg erkennen, welche in sechs der acht Spezifikationen in den Tabellen 6, 7 und 8 signifikant sind (Tabellen 3 und 4: positive Vorzeichen bei geringer Signifikanz). Kollinearität zwischen diesen Variablen und den Zolländerungs-Variablen implizieren, dass die Effekte dieser beiden Variablen evtl. einen gemeinsamen Ursprung haben.

Der Koeffizient für simulierte Änderung des technischen Fortschritts (P) oder der Produktivität haben insgesamt einen signifikant positiven Einfluss auf die Wohlfahrtsgewinne: Ein simulierter Schock, der die Produktionswerte um 1 Million US-Dollar steigen lässt, resultiert in einem korrespondierenden Wohlfahrtsgewinn von 86000 US-Dollar. Dieser Koeffizient stimmt nahezu exakt in allen Regressionen der Tabellen 6,7 und 8 überein und ist in allen drei Tabellen signifikant. In den Weltwohlfahrtsregressionen der Tabellen 3 und 4 beträgt dieser Koeffizient zwischen 170000 US-Dollar und 192000 US-Dollar je 1 Million technologiebedingtem Produktionswertanstieg weltweit über alle vier Regressionen bei signifikanten Koeffizienten.

Datenbasis (D): Erwartungsgemäß sind die Koeffizienten der BasisdatenDummy-Variablen negativ und die meisten sind statistisch signifikant. Das niedrigere Signifikanzniveau der ,anderen Datenbanken“ mag auf die Heterogenität dieser Variable zurückzuführen sein, welche alle Nicht-GTAP-Datenbanken erfasst. Die Verwendung der Datenbanken GTAP 4, GTAP 5 und GTAP 6 fuhrt zu einem entsprechend reduzierten simulierten Wohlfahrtsgewinn von 8,2 Mrd., 8,2 Mrd. und 10,7 Mrd. US-Dollar relativ zur GTAP 3 Datenbank. Dieses Muster ist bei leicht veränderten Koeffizienten konsistent für alle Spezifikationen in den Tabellen 3, 4, 6, 7 und 8.

Die Messung von Wohlfahrtsgewinnen ausgedrückt als Änderung des Bruttosozialprodukts (als Prozent oder absolut) (M) im Vergleich zu EV führt zu 7,2 Mrd. beziehungsweise 0,9 Milliarden US-Dollar höheren Wohlfahrtsgewinnen. Dabei ist der Effekt der prozentualen Änderungen signifikant und positiv, während der Effekt absoluter Bruttosozialproduktänderung statistisch nicht signifikant ist. Der Einfluss eines partiellen Wohlfahrtsmaßes (M) ist in den Tabellen 6, 7 und 8 negativ, in Tabelle 3 und Tabelle 4 jedoch positiv bei schwacher Signifikanz. Partielle Modelle (M) produzieren jedoch insgesamt größere Wohlfahrtsgewinne als allgemeine Gleichgewichtsmodelle, was 
insbesondere in den gewichteten Regressionen der Tabellen 6 und 8 signifikant ist. Eine genauere Trennung dieser Variablen, beispielsweise durch Einfürung studienspezifischer Dummies, könnte evtl. eine präzisere Messung des Koeffizienten für partielle Wohlfahrtsmaße ermöglichen.

Die Johansen-Macro-Closure (M) führt ebenfalls zu höheren simulierten Wohlfahrtsgewinnen, wobei der Effekt in drei von vier Spezifikationen in Tabelle 6 sowie in allen Spezifikationen der Tabellen 3 und 4 signifikant ist. Insbesondere in den gewichteten Regressionen mit Fixed Effects für unterschiedliche Länder (Tabelle 8) ist dieser Koeffizient hingegen nicht signifikant.

Die geschätzten Koeffizienten der 10 Interaktions-Dummies (M) weisen verschiedene Regelmäßigkeiten auf: Die geschätzten Koeffizienten für Modellspezifikationen mit hohen Armington-Elastizitäten sind wie erwartet positiv und signifikant, während nur zwei dieser Koeffizienten negativ und nicht signifikant sind. Die Kombination, welche nur bei den „mit B+L“ Observationen vorkommt, ist mit einem großen und signifikanten Koeffizienten assoziiert, welcher einen Teil der simulierten Wohlfahrtsgewinne aus diesen Experimenten absorbiert. Dies gilt auch für die Regressionen in den Tabellen 3, 4, 7 und 8.

Zusätzliche Restriktionen in der Angebotsseite (M) resultieren in signifikant niedrigeren Wohlfahrtsgewinnen (Tabelle 6: minus 2,3 Milliarden USDollar in Spalte 3; Tabelle 8: minus 6 Milliarden US-Dollar). Längere Projektionen der Datenbasis (M) vor der eigentlichen Simulation sind mit niedrigeren Wohlfahrtsgewinnen assoziiert, was sich insbesondere in den Weltwohlfahrtsregressionen (Tabellen 3 und 4), aber nicht in allen übrigen Spezifikationen als signifikant erweist. Die steigende Länge einer dynamischen Simulation (M) weist zunächst steigende und dann wieder fallende Wohlfahrtsgewinne auf. In der gewichteten Regression (Spalte 3 in Tabelle 6) ist letztgenannter Effekt dominierend für Simulationsergebnisse aus Experimenten, welche sich über einen Simulationszeitraum von mehr als 13 Jahren Simulationsdauer erstrecken. Dies trifft auch für beide gewichteten Regressionen in Tabelle 8 in gleicher Weise zu.

Fixierte Handelsbilanzen (M) sind wie erwartet (vgl. Kapitel 3.6.2) mit einem negativen Gewinn korreliert (Tabellen 3, 4, 6, 7 und 8) obwohl auch ein signifikantes, positives Vorzeichen in einer der ungewichteten Regressionen (Tabelle 6) auftaucht.

Ein Unterschied zwischen den Regressionen in Tabelle 6 einerseits sowie den Regressionen der Tabellen 3, 4, 7 und 8 andererseits besteht in der Abbildung der regionalen und sektoralen Aggregation (M): In Tabelle 6 ist nur ein Koeffizient für das Produkt aus der Zahl der aggregierten Regionen und der Zahl der aggregierten Sektoren ausgewiesen; in Tabelle 7 und Tabelle 8 sind diese Variablen, wie auch in Tabelle 3 und Tabelle 4, separat sowie als geschätzter Interaktionsterm ausgewiesen. Diese alternativen Spezifikationen wurden ge- 
wählt, um die Robustheit dieser evtl. von starker Multikollinearität betroffenen Koeffizienten durch unterschiedliche Datentransformationen überprüfen zu können.

Eine größere Disaggregation der Daten reduziert den simulierten Wohlfahrtsgewinn in Tabelle 6 (Spalte 3) signifikant. Dieser konsequent negative Einfluss einer stärkeren Disaggregation der Datenbasis auf die Wohlfahrtseffekte ist nicht deckungsgleich mit den zuvor formulierten Erwartungen, wonach eine verstärkte Aggregation die Wohlfahrtseffekte aufgrund des Durchschnitts- und Gewichtungsproblems unterschätzen sollte. In Tabelle 3 und Tabelle 4 sind die geschätzten Koeffizienten für die Zahl der Regionen meist positiv, jedoch ohne Signifikanz, aber der Koeffizient für die Zahl der Sektoren sowie der Interaktionseffekt beider Variablen weist ein negatives Vorzeichen auf und ist signifikant. In den durch Fixed Effects für jede geographische Region spezifizierten Regressionen in Tabelle 7 und Tabelle 8 sind jedoch alle Koeffizienten für die Zahl der Sektoren und die Zahl der Regionen positiv und bis auf wenige Ausnahmen signifikant. Der Interaktionsterm dieser beiden Variablen ist in den Tabellen 7 und 8 ebenfalls negativ und teilweise signifikant.

Insgesamt deuten die geschätzten Koeffizienten in den Tabellen 7 und 8 auf signifikanten Aggregations-Bias innerhalb des Literatursamples hin. Die marginalen Effekte dieses Aggregations-Bias sind jedoch im Vergleich zum allgemeinen Unterschied zwischen Datenbanken (durch Preise, Wechselkurse, etc.) gering, wobei sich durch größere Disaggregationen der Datenbasen dennoch beträchtliche absolute Unterschiede zwischen Simulationsexperimenten ergeben können. Die Bedeutung unterschiedlicher Basisdaten (GTAP 6 gegenüber allen früheren GTAP-Versionen oder Nicht-GTAP-Datensätzen) sowie das Problem des Aggregations-Bias im Bereich von Zöllen wird unter Modellierern jüngst wieder stark diskutiert (vgl. z.B. Anderson und Neary 2003, Martin, van der Mensbrugghe und Manole 2003, Anderson 2006, Bouët und Krasniqi 2006, Bchir, Jean, und Laborde 2006, Brockmeier und Pelikan 2006, Pelikan und Brockmeier 2007).

Die Zahl der aggregierten Agrarprodukte (M) innerhalb der Zahl der Sektoren ist in allen Spezifikationen der Tabellen 3, 4, 6, 7 und 8 bei einem vergleichsweise geringen marginalen Effekt signifikant bei negativem Vorzeichen. Dies ist insbesondere durch unterschiedliche Aggregationen anhand der GTAPDatenbank zu erklären, da einige Autoren die GTAP-Sektoren für primäre und verarbeitete Agrarprodukte zusammenfassen. Die resultierenden Sektoren bringen die hohen Zölle der primären Agrarprodukte mit den z.T. sehr großen Handelsmengen (in Mio. US-Dollar) der verarbeiteten Nahrungsmittel zusammen, wodurch hoch protektionierte, große, einzelne Sektoren entstehen. Eine stärkere Disaggregation verarbeiteter und primärer Agrarprodukte bewirkt daher c.p. innerhalb des Literatursamples niedrigere Wohlfahrtseffekte. 
Sofern Autoren eine ad hoc Modifikation gebräuchlicher Elastizitäten (M) erwähnen, ist dies mit signifikant größeren Wohlfahrtsgewinnen (Spalte 3, Tabelle 6), aber mit signifikant negativen Wohlfahrtsgewinnen in den Tabellen 3, 4 und 8 assoziiert. Verwenden Autoren hingegen Elastizitäten, welche auf eigenen ökonometrischen Schätzungen basieren, fallen die simulierten Wohlfahrtseffekte signifikant niedriger aus (höher in Tabellen 3, 4 und 8). Dies könnte erklärt werden, indem man annimmt, dass ad hoc Modifikationen tendenziell von denjenigen durchgeführt werden, die konventionell verwendete Werte und ökonomische Schätzungen für zu hoch halten, um die tatsächliche Größenordnung vermuteter Wohlfahrtsgewinne zu schätzen. Die Ergebnisse von Harrison, Rutherford und Tarr (1997), Gehlhar (1997) sowie Feenstra (2006) zeigen zudem, dass ökonometrische Schätzungen wichtiger Elastizitäten (z.B. Armington) eventuell zu höheren als den gemeinhin angenommene Werten führen. Diese Interpretation sollte ohne eingehende Prüfung der empirischen Literatur jedoch nicht verallgemeinert werden, da sich die zitierten Studien stets nur auf bestimmte geographische Regionen beziehen. 
Tabelle 5: Deskriptive Statistik der Variablen innerhalb der Meta-Regression für simulierte regionale Wohlfahrtsänderungen.

\begin{tabular}{|c|c|c|c|c|c|c|c|c|c|c|}
\hline \multirow[t]{2}{*}{ Kat. } & \multirow[t]{2}{*}{ Variable } & \multirow[t]{2}{*}{ Einheit } & \multicolumn{4}{|c|}{$\begin{array}{l}\text { Stichprobe ohne } B+L^{2} \\
\text { ( } 107 \text { Studien, } n=5395 \text { ) }\end{array}$} & \multicolumn{4}{|c|}{$\begin{array}{l}\text { Stichprobe mit } B+L^{\prime \prime} \\
\text { (110 Studien, } n=5835 \text { ) }\end{array}$} \\
\hline & & & Min. & Max. & Mittel & Std. Abw. & Min. & Max. & Mittel & Std. Abw. \\
\hline $\mathbf{Y}$ & Simulierte Wohlfahrtsănnderung & Mio. USS & -43930 & 203400 & 3472 & 10914 & -43930 & 859400 & 5007 & 25967 \\
\hline $\mathbf{M}$ & Abhängige Variable: prozentuale Änderung des GDP & Dummy & 0 & 1 & 0,045 & - & 0 & 1 & 0,040 & - \\
\hline $\mathbf{M}$ & Abhängige Variable: absolute Änderung des GDP & Dummy & 0 & 1 & 0,086 & -1 & 0 & 1 & 0,080 & - \\
\hline$M$ & Abhängige Variable: PE Wohlfahrt & Dummy & 0 & 1 & 0,122 & - & 0 & 1 & 0,110 & - \\
\hline $\mathbf{M}$ & Partielles Gleichgewichtsmodell (Dummy PE=1) & Dummy & 0 & 1 & 0,171 & - & 0 & 1 & 0,158 & - \\
\hline $\mathbf{M}$ & Modell mit Johansen-Macro-Closure (Dummy ja=1) & Dummy & 0 & 1 & 0,010 & - & 0 & 1 & 0,079 & - \\
\hline $\mathbf{M}$ & Dynam., CRTS, perf. Wettb., fixer Kapitalst., hohe Arm.-El. & Dummy & 0 & 1 & 0,014 & - & 0 & 1 & 0,013 & - \\
\hline $\mathbf{M}$ & Dynam., CRTS, perf. Wettb., fixer Kapitalst., niedrige Arm.-El. & Dummy & 0 & 1 & 0,080 & - & 0 & 1 & 0,057 & - \\
\hline $\mathbf{M}$ & Dynam., CRTS, perf. Wettb., steig. Kapitalst., hohe Arm.-El. & Dummy & 0 & 1 & 0,061 & - & 0 & 1 & 0,074 & - \\
\hline $\mathbf{M}$ & Dynam., IRTS, imperf. Wettb., steig. Kapitalst., niedrige Am.-El. & Dummy & 0 & 1 & 0,028 & - & 0 & 1 & 0,026 & - \\
\hline M & Dynam., IRTS, imperf. Wettb., steig. Kapitalst., hohe Arm.-El. & Dummy & 0 & 1 & 0,003 & - & 0 & 1 & 0,003 & - \\
\hline $\mathbf{M}$ & Komp. stat., CRTS, perf. Wettb., steig. Kapitalst., niedr. Arm.-El. & Dummy & 0 & 1 & 0,132 & - & 0 & 1 & 0,120 & - \\
\hline $\mathbf{M}$ & Komp. stat., CRTS, perf. Wettb., steig. Kapitalst., hohe Arm.-El. & Dummy & 0 & 1 & 0,106 & - & 0 & 1 & 0,098 & - \\
\hline $\mathbf{M}$ & Komp. stat., IRTS, imperf. Wettb., fixer Kapitalst., hohe Arm.-El. & Dummy & 0) & 1 & 0,000 & - & 0 & 1 & 0,070 & - \\
\hline$M$ & Komp. stat., IRTS, imperf. Wettb., steig. Kap., niedr. Arm.-El. & Dummy & 0 & 1 & 0,070 & - & 0 & 1 & 0,060 & - \\
\hline Mh & Komp. stat., IRTS, imperf. Wettb., steig. Kapitalst., hohe Arm.-E. & Dummy & 0 & 1 & 0,021 & - & 0 & 1 & 0,020 & - \\
\hline M & Zusătzliche Beschränkungen von Faktormobilităt/-angebot & Dummy & 0 & 1 & 0,240 & - & 0 & 1 & 0,220 & - \\
\hline$N_{3}$ & Pre-simulative Datenprojektion: Zeitraum in Jahren & Jahre & 0 & 14 & 1,890 & 3,86 & 0 & 14 & 1,820 & 3,85 \\
\hline$M_{0}$ & Dynamische Modelle: Länge der Simulationsdauer in Jahren & Jahre & 0 & 28 & 4,290 & 5,91 & 0 & 28 & 4,290 & 5,91 \\
\hline$M$ & Handelsgleichgewicht eines oder mehrerer Länder ist fix & Dummy & 0 & 1 & 0,050 & - & 0 & 1 & 0,100 & - \\
\hline$M$ & Anzahl der Regionen*Anzahl der Sektoren & Anzahl & 1 & 1665 & 354,1 & 246.4 & 1 & 1665 & 355,5 & 237,1 \\
\hline Nes & Anzahl der aggregierten Agrarprodukte & Anzahl & 1 & 158 & 17,4 & 31,9 & 1 & 158 & 16,1 & 31,0 \\
\hline $\mathbf{M}$ & Autor erwăhnt ad hoc Modifikationen verwendeter Elastizităten & Dummy & 0 & 1 & 0,015 & - & 0 & 1 & 0,010 & - \\
\hline M & Autor benutzt Elastizitäten aus eigenen okonometr. Schätzungen & Dummy & 0 & 1 & 0,020 & - & 0 & 1 & 0,020 & - \\
\hline $\mathbf{P}_{1}$ & Änderung von Zöllen, Exp.-Subv. und Amber-Box Maßnahmen & Mio. US\$ & -2506000 & 257000 & -49920 & 144463 & -12790000 & 372400 & -63200 & 321306 \\
\hline $\mathbf{P}_{1}^{c}$ & Änderungen von Non-Tariff-Barriers & Mio. USS & -3794000 & 0 & -15320 & 128064 & -3794000 & 0 & -15540 & 126644 \\
\hline$P \omega$ & Änderungen von Blue/Green-Box Maßnahmen & Mio. US\$ & -6898000 & 726700 & -50500 & 324142 & -6898000 & 726700 & -50500 & 317577 \\
\hline $\overrightarrow{P i}$ & Handelsvolumen eines Landes (Summe Sektoren in Modell) & Mio. USS & 0 & 2570000 & 181600 & 278604 & 0 & 2570000 & 183100 & 278797 \\
\hline $\mathrm{POr}$ & Simulierte Änderung von technischem Fortschritt o.Ä. & Mio. USS & -441000 & 11050 & $-189,4$ & 7153 & -441000 & 11050 & $-175,2$ & 6879 \\
\hline Do & Datenbasis GTAP-4 & Dummy & 1 & 0 & 0,112 & - & 1 & 0 & 0,145 & - \\
\hline $\mathrm{R}$ & Datenbasis GTAP-5 & Dummy & 1 & 0 & 0,353 & - & 1 & 0 & 0,355 & - \\
\hline D & Datenbasis GTAP-6 & Dummy & 1 & 0 & 0,275 & - & 1 & 0 & 0,260 & - \\
\hline D & Nicht-GTAP Datenbasis & Dummy & 1 & 0 & 0,182 & -1 & 1 & 0 & 0,169 & t \\
\hline
\end{tabular}


Tabelle 6: OLS-Regressionsergebnisse (robuste Standardfehler) der Meta-Regression (siehe Erläuterung im Text).

\begin{tabular}{|c|c|c|c|c|c|c|c|c|c|c|c|c|}
\hline \multirow{3}{*}{$\begin{array}{r}\text { Gewichtungsschema } \rightarrow \\
\text { Stichprobe } \rightarrow \\
\end{array}$} & \multicolumn{6}{|c|}{ Ungewichtet } & \multicolumn{6}{|c|}{ Gewichtet mit [1/(Beobachtungen je Studie)] } \\
\hline & \multicolumn{3}{|c|}{ ohne $B+L^{\circ}$} & \multicolumn{3}{|c|}{ mit $B+L^{3}$} & \multicolumn{3}{|c|}{ ohne $\mathrm{B}+\mathrm{L}^{*}$} & \multicolumn{3}{|c|}{ mit $\mathrm{B}+\mathrm{L}^{\mathrm{a}}$} \\
\hline & Koeff. & & St. Fehl. & Koeff. & & St. Fehl. & Koeff. & & St. Fehl. & Koeff. & & St. Fehl. \\
\hline Achsenabschnitt & 5223 & $+* *$ & 965 & 1566 & & 1450 & 9335 & *** & 1005 & 7158 & $* * *$ & 594 \\
\hline Abhängige Variable: prozentuale Änderung des GDP & 2205 & ** & 1068 & 2881 & ** & 1304 & 7236 & $* * *$ & 822 & 6226 & $* * *$ & 394 \\
\hline Abhängige Variable: absolute Änderung des GDP & 3502 & $* * *$ & 897 & 3192 & $* * *$ & 981 & 886 & & 1338 & -564 & & 571 \\
\hline Abhängige Variable: PE Wohlfahrt & -6112 & $* * *$ & 2353 & -4724 & * & 2811 & -2187 & *** & 601 & -2168 & $* *$ & 282 \\
\hline Partielles vs. allgemeines Gleichgewicht (Dummy PE=1) & 3761 & & 3616 & 887 & & 4392 & 2339 & *** & 803 & 266 & & 623 \\
\hline Modell mit Johansen-Macro-Closure (Dummy ja $=1$ ) & 2569 & & 2061 & 7938 & $* * *$ & 3039 & 2138 & $*$ & 1141 & 2633 & **** & 894 \\
\hline Dynam., CRTS, perf. Wettb., fixe & 4589 & $* * *$ & 1295 & 3578 & $* * *$ & 1363 & 9419 & $* * *$ & 2741 & 8124 & $* * *$ & 859 \\
\hline Dynam., CRTS, perf. Wettb., s & -1363 & & 836 & -2556 & ** & 1132 & 3357 & $* * *$ & 1028 & 2781 & $* * *$ & 511 \\
\hline Dynam., CRTS, perf. Wettb., st & 4950 & $* * *$ & 811 & 3982 & $* * *$ & 1088 & 17335 & $* * *$ & 1366 & 18628 & $* * *$ & 1260 \\
\hline Dynam., IRTS, imperf. Wettb., steig. Kapitalst, niedrige Arm.-El. & 1455 & * & 798 & -198 & & 1127 & 3970 & * & 2109 & 1126 & & 1229 \\
\hline Dynam., IRTS, imperf. Wettb, steig. Kapitalst., hohe Am.-El. & 8215 & & 5626 & 6823 & & 5744 & 9063 & $* * *$ & 2857 & 5675 & $* * *$ & 1616 \\
\hline Komp. stat., CRTS, perf. Wettb., steig. Kap., niedrige Arm.-El. & 434 & & 446 & 673 & & 615 & 1249 & $* * *$ & 446 & 1038 & **** & 276 \\
\hline Komp. stat., CRTS, perf. Wettb., steig. Kapitalst., hohe Arm.-El. & -709 & & 803 & -290 & & 1160 & 3185 & ** & 1609 & 2518 & $* *$ & 860 \\
\hline Komp. stat., IRTS, imperf. Wettb., fixer Kapitalst., hohe Arm.-El. & & & & 12330 & $* * *$ & 4012 & & & & 19568 & $* * *$ & 5398 \\
\hline niedrige Arm.-El. & 2249 & $* * *$ & 705 & 1999 & ** & 797 & 6224 & $* * *$ & 1257 & 4631 & $* * *$ & 560 \\
\hline alst., hohe Arm.-El. & 6662 & $* * *$ & 1951 & 6958 & * & 3670 & 8288 & ** & 3946 & 6219 & $*$ & 3383 \\
\hline ität/-angebot & 413 & & 478 & 247 & & 708 & -2336 & **** & 527 & -1940 & $* * *$ & 327 \\
\hline nulative Dat & -66 & * & 36 & -70 & & 58 & -15 & & 93 & -172 & ** & 71 \\
\hline Jahren & -633 & *** & 169 & -472 & ** & 198 & -388 & $* * *$ & 112 & .443 & $* * *$ & 71 \\
\hline Jahren $]^{\wedge} 2$ & 44 & $* * *$ & 12 & 38 & $* * *$ & 14 & 29 & $* * *$ & 7 & 36 & $* * *$ & 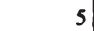 \\
\hline Länder ist fix & 1908 & ** & 816 & -3854 & & 3035 & -2674 & $* * *$ & 617 & -4265 & $* * *$ & 1174 \\
\hline Anzahl der Regior & -3.1 & **** & 0.6 & $-0,593$ & & 1,1 & $-6,0$ & $* * *$ & 0.9 & $-5,5$ & $* * *$ & 0.8 \\
\hline Anzahl der aggreg & -45.4 & ** & 18,4 & $-38,4$ & $*$ & 22,3 & $-73,2$ & $* * *$ & 12,7 & $-62,9$ & $* * *$ & 8,5 \\
\hline rwahnt ad hoc & -1799 & $* * *$ & 645 & -775 & & 1404 & 3136 & $* * *$ & 959 & 2198 & $* *$ & 869 \\
\hline ökonometr. Schătzungen & -462 & & 617 & 1327 & & 1113 & -2859 & $* * *$ & 403 & -537 & * & 292 \\
\hline bv. und Amber-Box MaBnahmen & -0.017 & ** & 0,007 & $-0,041$ & *** & 0,011 & $-0,016$ & **** & 0.004 & $-0,056$ & *** & 0.007 \\
\hline dênderung von Zöllen, Exp. & $8,0 \mathrm{E}-9$ & & 9,9E-9 & $-2,6 E-9$ & $* *$ & $7,6 \mathrm{E}-10$ & $2,5 \mathrm{E}-8$ & **** & $2,2 \mathrm{E}-9$ & $-3,6 \mathrm{E}-9$ & *** & $1,1 \mathrm{E}-9$ \\
\hline Äuderungen von Non-Tar & -0.015 & ** & 0,006 & $-0,011$ & ** & 0,006 & $-0,008$ & & 0,009 & $-0,004$ & & 0,004 \\
\hline Araderungen von Blue/Green-Bo: & 0.001 & * & 0,001 & 0,005 & ** & 0,002 & 0,005 & *** & 0,002 & 0,001 & & 0,002 \\
\hline Handelsvolumen eines Landes (Summe Sektoren in Modell) & 0.007 & *** & 0,001 & 0,015 & *** & 0,003 & 0,009 & $* * *$ & 0,001 & 0,014 & *** & 0.002 \\
\hline Simulierte Änderung von technischem Fortschritt o. $\vec{A}$. & 0.085 & $* * *$ & 0,019 & 0,086 & *** & 0.018 & 0,086 & $* * *$ & 0,020 & 0,086 & *** & 0.020 \\
\hline Gatenbasis GTAP-4 & -1250 & & 926 & -1987 & & 1325 & .8205 & $* * *$ & 1214 & -8443 & *** & 708 \\
\hline Ratenbasis GTAP-5 & -3959 & $* * *$ & 961 & -3411 & $* *$ & 1240 & -8171 & $* * *$ & 1059 & -8468 & $* * *$ & 745 \\
\hline Datenbasis GTAP-6 & -4682 & $* * *$ & 945 & -4584 & $* * *$ & 1137 & -10696 & $* * *$ & 1335 & -11199 & $* * *$ & 729 \\
\hline Nicht-GTAP Datenbasis & -690 & & 2221 & 3663 & & 2598 & .3104 & $* * *$ & 1010 & -1169 & $*$ & 549 \\
\hline $\begin{array}{l}\text { Freiheitsgrade } \\
\text { Korrigiertes } \mathbf{R}^{2}\end{array}$ & & $\begin{array}{l}5361 \\
0,38\end{array}$ & & & $\begin{array}{l}5800 \\
0,19\end{array}$ & & & $\begin{array}{l}5361 \\
0,67\end{array}$ & & & $\begin{array}{l}5800 \\
0,46\end{array}$ & \\
\hline
\end{tabular}


Tabelle 7: OLS-Regressionsergebnisse (robuste Standardfehler, ungewichtet) der Meta-Regression; Dummies für jede aggregierte Region.

\begin{tabular}{|c|c|c|c|c|c|c|c|c|c|c|}
\hline \multirow{3}{*}{$\begin{array}{rr}\text { Gewichtungsschema } \rightarrow \\
\text { Stichprobe } \rightarrow\end{array}$} & \multicolumn{10}{|c|}{ Ungewichtet } \\
\hline & \multicolumn{5}{|c|}{ mit $B+L^{3}$} & \multicolumn{5}{|c|}{ ohne $\mathrm{B}+\mathrm{L}^{2}$} \\
\hline & Koeff. & Std. Fehl. & $t$-Wert & $\operatorname{Pr}(>t)$ & & Koeff. & Std. Febl. & $t$-Wert & $\operatorname{Pr}(>|t|)$ & \\
\hline Achsenabschnitt & -1788 & 3192 & $-0,560$ & 0,575 & & -2594 & 2776 & $-0,934$ & 0,350 & \\
\hline Abhängige Variable: prozentuale Änderung des GDP & 5736 & 1762 & 3,255 & 0,001 & $* *$ & 3419 & 1474 & 2,320 & 0,020 & ** \\
\hline Abhlingige Variable: absolute Änderung des GDP in Mio. USS & 2656 & 969.4 & 2.740 & 0,006 & $* *$ & 2501 & 895.9 & 2.792 & 0,005 & $* * *$ \\
\hline Abhalngige Variable: PE Wohlfahrt & -9365 & 4274 & $-2,192$ & 0,028 & ** & -8008 & 3275 & -2.445 & 0,015 & $*$ \\
\hline Modell: Partielles vs. allgemeines Gleichgewicht ( $\mathrm{PE}=1$ ) & 4740 & 5239 & 0,905 & 0,366 & & 8014 & 3900 & 2,055 & 0,040 & ** \\
\hline Modell verwendet Johansen-Macro-Closure (I =्रja) & 9207 & 4221 & 2,181 & 0,029 & ** & 1544 & 2193 & 0,704 & 0,482 & \\
\hline Dyn., CRTS, vollk. Wettb., fixer Kapitalst., hohe Árm.-El. & 5658 & 1458 & 3,880 & 0,000 & *** & 3971 & 1186 & 3,349 & 0,001 & *** \\
\hline Dyn., CRTS, vollk. Wettb., steig. Kapitalst.. niedrige Arm.-El. & $-488,8$ & 1392 & $-0,351$ & 0,726 & & -1715 & 847.9 & -2.023 & 0,043 & $*$ \\
\hline Dyn., CRTS, vollk. Wettb., steig. Kapitalst., hohe Arm.-El. & 4835 & 1184 & 4,085 & 0,000 & *** & 3466 & 733,5 & 4,725 & 0,000 & *** \\
\hline Dyn., IRTS, unvollk. Wettb., steig. Kapitalst., niedrige Am.-El. & 990,1 & 1405 & 0,704 & 0,481 & & $-137,2$ & 998,3 & $-0,137$ & 0,891 & \\
\hline Dyn., IRTS, unvollk. Wettb. steig. Kapitalst., hohe Arm.-El. & 13024 & 5934 & 2,195 & 0,028 & ** & 11201 & 5805 & 1,929 & 0,054 & * \\
\hline Komp. stat., CRTS, vollk. Wettb., steig. Kapitalst., niedr. Arm.-El. & 651.7 & 610.6 & 1.067 & 0.286 & & 295.2 & 466,3 & 0.633 & 0,527 & \\
\hline Komp. stat., CRTS, vollk. Wettb., steig. Kapitalst., hohe Arm.-El. & 548,7 & 1286 & 0,427 & 0,670 & & -1261 & 755,7 & $-1,668$ & 0,095 & * \\
\hline Komp. stat., IRTS, unvollk. Wettb., fixer Kapitalst., hohe Arm.-El. & 14980 & 4278 & 3,502 & 0,000 & $* * *$ & & -1 & & & \\
\hline Komp. stat., IRTS, unvollk. Wettb., steig. Kapitalst., niedr. Arm.-El. & 2236 & 950,4 & 2,353 & 0,019 & ** & 2332 & 729,6 & 3,196 & 0,001 & $* *$ \\
\hline Komp. stat., IRTS, unvollk. Wettb., steig. Kapitalst., hohe Arm.-El. & 6324 & 3165 & 1,998 & 0,046 & ** & 5853 & 1580 & 3,705 & 0,000 & $* * *$ \\
\hline Zusäzliche Beschrankungen von Faktormobilität/-angebot & 600.9 & 768.7 & 0,782 & 0,434 & & 686.1 & 504.4 & 1,360 & 0,174 & \\
\hline Pre-simulative Datenprojektion: Zeitraum in Jahren & $-63,40$ & 67,95 & $-0,933$ & 0,351 & & $-49,35$ & 37,74 & $-1,308$ & 0,191 & \\
\hline Dypamische Modelle: Lănge der Simulationsdauer in Jahren & $-752,0$ & 240,5 & $-3,127$ & 0,002 & $\cdots$ & $-594,0$ & 190,2 & $-3,122$ & 0,002 & $+* *$ \\
\hline [Dynamische Modelle: Lange der Simulationsdauer in Jahren $]^{\wedge} 2$ & 53,81 & 15,45 & 3,482 & 0,001 & ** & 44,82 & 13,67 & 3,280 & 0,001 & *** \\
\hline Họndelsbilanz eines oder mehrerer Lander ist fix & -6991 & 3663 & $-1,909$ & 0,056 & $*$ & 1839 & 927.9 & 1,982 & 0.048 & $* *$ \\
\hline Afíahl der Regionen & 11.78 & 12.18 & 0,967 & 0,334 & & 6.987 & 9.202 & 0.759 & 0,448 & \\
\hline Apipahl der Sektoren & 113,1 & 61,44 & 1,841 & 0,066 & $*$ & 179,3 & 58,09 & 3,087 & 0,002 & *** \\
\hline Anzahl der aggregierten Agrarprodukte & $-73,19$ & 34.87 & -2.099 & 0.036 & ** & $-47,97$ & 25,60 & $-1,874$ & 0,061 & * \\
\hline Regionen*Sektoren & -1.985 & 2.059 & -0.964 & 0.335 & & -5.940 & 1.585 & -3.746 & 0,000 & *** \\
\hline Affifor erwhihnt ad hoc Modifikationen verwendeter Elastizităten & 1213 & 1414 & 0,857 & 0.391 & & -892.6 & 701.3 & -1.273 & 0,203 & \\
\hline Autor benutzt Elastizitaten aus eigenen okonometr. Schătzungen & 2280 & 1337 & 1,705 & 0,088 & * & 0,294 & 683,4 & 0,000 & 1,000 & \\
\hline Ärgerung von Zollen, Exp.-Subv. und Amber-Box MaBnahmen & $-0,033$ & 0,010 & $-3,450$ & 0,001 & $* * *$ & $-0,024$ & 0,004 & $-6,040$ & 0,000 & *** \\
\hline [ÄAouderung von Zollen, Exp.-Subv. und Amber-Box MaBnahmen]^2 & 0,000 & 0,000 & $-2,955$ & 0,003 & $* * *$ & 0,000 & 0,000 & $-1,864$ & 0,062 & * \\
\hline Anderungen von Non-Tariff-Barriers & -0.013 & 0.004 & $-3,091$ & 0,002 & $* *$ & -0.022 & 0.004 & -6.087 & 0,000 & *** \\
\hline Äderungen von Blue/Green-Box Maßnahmen & 0,004 & 0,002 & 2,324 & 0,020 & ** & 0,001 & 0,001 & 1,272 & 0,204 & \\
\hline Simulierte Ändenung von technischem Fortschritt o.A. & 0,091 & 0,026 & 3,449 & 0,001 & $* *$ & 0,088 & 0,025 & 3,576 & 0,000 & $* * *$ \\
\hline Datenbasis GTAP 4 & -2184 & 1386 & $-1,576$ & 0,115 & & -1961 & 963,2 & $-2,036$ & 0,042 & $* *$ \\
\hline Dagęenbasis GTAP-5 & -4126 & 1305 & $-3,162$ & 0,002 & $* * *$ & -4344 & 924.3 & -4.699 & 0,000 & $* * *$ \\
\hline Dáenbasis GTAP-6 & -5723 & 1204 & $-4,755$ & 0,000 & $* * *$ & -4652 & 894,5 & $-5,201$ & 0,000 & $* * *$ \\
\hline Nicht-GTAP Datenbasis & 3004 & 2647 & 1,135 & 0,257 & & -2338 & 1910 & $-1,224$ & 0,221 & \\
\hline $\begin{array}{l}\text { [335 Dummies fur regionale Aggregationen siche Anhang] } \\
\text { Residual Standard Error / Korrigiertes } \text { R }^{2}\end{array}$ & & & $0 / 0,221$ & & & & & $10,7>00$ & & \\
\hline
\end{tabular}


Tabelle 8: OLS-Regressionsergebnisse (robuste Standardfehler, gewichtet) der Meta-Regression; Dummies für jede aggregierte Region.

\begin{tabular}{|c|c|c|c|c|c|c|c|c|c|c|}
\hline \multirow{3}{*}{$\begin{array}{|lr|} & \text { Gewichtungsschema } \rightarrow \\
& \text { Stichprobe } \rightarrow \\
\text { IVariable } & \end{array}$} & \multicolumn{10}{|c|}{ Gewichtet mit [1/(Beobachtungen in der Studie)] } \\
\hline & \multicolumn{5}{|c|}{ mit $B+L^{3}$} & \multicolumn{5}{|c|}{ ohne $B+L^{2}$} \\
\hline & Koeff. & Std. Fehl. & t-Wert & $\operatorname{Pr}(>|t|)$ & & Koeff. & Std. Fehl. & t-Wert & $\operatorname{Pr}(>|t|)$ & \\
\hline Achsenabschnitt & -9101 & 750,3 & $-12,13$ & 0,000 & $* * *$ & -9799 & 1547 & $-6,334$ & 0,000 & *** \\
\hline Abhăngige Variable: prozentuale Änderung des GDP & 4703 & 442,3 & 10,63 & 0,000 & *** & 4556 & 977,4 & 4,662 & 0,000 & *** \\
\hline Abhängige Variable: absolute Änderung des GDP in Mio. USS & -1526 & 470,1 & $-3,247$ & 0,001 & *** & -1589 & 1123 & $-1,415$ & 0,157 & \\
\hline Abhängige Variable: PE Wohlfahrt & .9838 & 646.0 & $-15,23$ & 0.000 & *** & -9182 & 1165 & $-7,885$ & 0,000 & *** \\
\hline Modell: Partielles vs. allgemeines Gleichgewicht $(\mathrm{PE}=1)$ & 20726 & 718,7 & 28,84 & 0,000 & *** & 21351 & 1681 & 12,70 & 0,000 & *** \\
\hline Modell verwendet Johansen-Macro-Closure $(1=\mathrm{ja})$ & 1673 & 1530 & 1,093 & 0,274 & & $-787,1$ & 1641 & $-0,480$ & 0,631 & \\
\hline Dyn., CRTS, vollk. Wettb., fixer Kapitalst., hohe Arm.-El. & 12342 & 939.2 & 13,14 & 0,000 & *** & 11311 & 2098 & 5.393 & 0,000 & *** \\
\hline Dyn., CRTS, vollk. Wettb., steig. Kapitalst., niedrige Arm.-El. & 2106 & 723.1 & 2,913 & 0.004 & *** & 1280 & 1018 & 1.257 & 0,209 & \\
\hline Dyn., CRTS, vollk. Wettb., steig. Kapitalst., hohe Arm.-El. & 12290 & 706.9 & 17,39 & 0.000 & $* * *$ & 11441 & 988.4 & 11.58 & 0,000 & *** \\
\hline Dyn., IRTS, unvollk. Wettb., steig. Kapitalst., niedrige Am.-El. & 450,5 & 1102 & 0,409 & 0,683 & & $.156,3$ & 1989 & $-0,079$ & 0,937 & \\
\hline Dyn., IRTS, unvollk. Wettb, steig. Kapitalst., hohe Arm.-El. & 14483 & 1863 & 7,773 & 0,000 & *** & 13323 & 2684 & 4,965 & 0,000 & *** \\
\hline Komp. stat., CRTS, vollk. Wettb., steig. Kapitalst., niedr. Arm.-El. & 4518 & 278,4 & 16,23 & 0,000 & *** & 4314 & 531.0 & 8,124 & 0,000 & *** \\
\hline Komp. stat., CRTS, vollk. Wettb., steig. Kapitalst., hohe Arm.-El. & 4708 & 830.4 & 5,670 & 0,000 & *** & 3828 & 1310 & 2.921 & 0,004 & *\#* \\
\hline Komp. stat., IRTS, unvollk. Wettb., fixer Kapitalst., hohe Arm.-El. & 20746 & 4766 & 4,353 & 0,000 & $* * *$ & & & & & \\
\hline Komp. stat., IRTS, unvollk. Wettb., steig. Kapitalst., niedr. Arm.-El. & 5717 & 554,5 & 10,31 & 0,000 & *** & 5972 & 1173 & 5,090 & 0,000 & *** \\
\hline Komp. stat.. IRTS, unvollk. Wettb., steig. Kapitalst., hohe Arm.-El. & 6090 & 2586 & 2.355 & 0,019 & ** & 5716 & 2747 & 2.081 & 0,038 & ** \\
\hline Zusătzliche Beschränkungen von Faktormobilität/-angebot & -6104 & 382.0 & $-15,98$ & 0.000 & *** & -6087 & 538.9 & -11.30 & 0,000 & *** \\
\hline PAls-simulative Datenprojektion: Zeitraum in Jahren & 31,84 & 51,98 & 0,612 & 0,540 & & 24,81 & 67,89 & 0,365 & 0,715 & \\
\hline Dofrnamische Modelle: Länge der Simulationsdauer in Jahren & $-798,3$ & 97,00 & $-8,229$ & 0,000 & *** & $-625,2$ & 108,7 & $-5,752$ & 0,000 & *** \\
\hline [Dynamische Modelle: Luange der Simulationsdauer in Jahren]^2 & 58,31 & 4,899 & 11,90 & 0,000 & $* * *$ & 48,47 & 6,097 & 7,951 & 0,000 & $* * *$ \\
\hline Hĭndelsbilanz eines oder mehrerer Länder ist fix & -4553 & 1524 & $-2,987$ & 0,003 & *** & -1314 & 624,7 & $-2,103$ & 0,035 & ** \\
\hline Añzahl der Regionen & 35.24 & 5,915 & 5,958 & 0,000 & $* * *$ & 40,20 & 9,242 & 4,350 & 0,000 & $* * *$ \\
\hline Anzahl der Sektoren & 369,3 & 13,32 & 27,73 & 0,000 & *** & 388,7 & 27,30 & 14,24 & 0,000 & **** \\
\hline Alozahl der aggregierten Agrarprodukte & $-164,1$ & 9,540 & -17.20 & 0,000 & *** & $-152,6$ & 16,82 & $-9,073$ & 0,000 & $* * *$ \\
\hline Regionen*Sektoren & $-7,511$ & 0,874 & $-8,598$ & 0,000 & *** & $-9,557$ & 0,890 & $-10,73$ & 0,000 & *** \\
\hline Agtor erwahhnt ad hoc Modifikationen verwendeter Elastizitäten & -1938 & 913.1 & $-2,122$ & 0,034 & ** & -3596 & 1010 & $-3,560$ & 0,000 & *** \\
\hline Antor benutzt Elastizităten aus eigenen ökonometr. Schătzungen. & 1133 & 424,1 & 2,672 & 0,008 & **** & 657,9 & 682,1 & 0,965 & 0,335 & \\
\hline Änderung von Zöllen, Exp.-Subv. und Amber-Box MaBnahmen & $-0,031$ & 0,008 & $-3,598$ & 0,000 & *** & $-0,022$ & 0,006 & $-3,844$ & 0,000 & $* * *$ \\
\hline [Änderung von Zollen, Exp.-Subv. und Amber-Box MaBnahmen] ${ }^{\wedge} 2$ & 0,000 & 0,000 & $-2,704$ & 0,007 & *** & 0,000 & 0,000 & $-0,121$ & 0,904 & \\
\hline Änderungen von Non-Tariff-Barriers & -0.013 & 0.003 & $-3,617$ & 0.000 & $* * *$ & $-0,017$ & 0,005 & $-3,495$ & 0,000 & *** \\
\hline Änderungen von Blue/Green-Box Maßnahmen & 0,003 & 0.001 & 2,754 & 0.006 & $* * *$ & 0,002 & 0,001 & 2,182 & 0,029 & ** \\
\hline Sfgulierte Änderung von technischem Fortschritt o.Ä. & 0,085 & 0,009 & 9,827 & 0,000 & $* * *$ & 0,085 & 0,022 & 3,849 & 0,000 & $* *$ \\
\hline Drenbasis GTAP-4 & -2060 & 821,4 & $-2,509$ & 0,012 & ** & .1847 & 1261 & $-1,465$ & 0,143 & \\
\hline Datenbasis GTAP-5 & -5092 & 661.1 & $-7,703$ & 0.000 & *** & -4418 & 1208 & -3.656 & 0,000 & $* * *$ \\
\hline Datenbasis GTAP-6 & -6751 & 794.3 & $-8,499$ & 0.000 & $* * *$ & -5716 & 1359 & $-4,205$ & 0,000 & $* *$ \\
\hline Nicht-GTAP Datenbasis & -3684 & 963,7 & $-3,823$ & 0,000 & $* * *$ & -5552 & 1380 & $-4,023$ & 0,000 & **** \\
\hline $\begin{array}{l}\text { [335 Dummies für regionale Aggregationen siehe Anhang, } \\
\text { Residual Standard Error / Korrigiertes } \text { R }^{2}\end{array}$ & & & 0.5479 & & & & & 8407 & & \\
\hline
\end{tabular}


Anmerkung zu den Tabellen: Die Variablen sind in Tabelle 2 erläutert. *, ** und *** entsprechen einem Signifikanzniveau von 10,5 und $1 \%$. Die Standardfehler sind heteroskedastisch konsistent. (Die Homoskedastizitätshypothese war durch den BreuschPagan Test verworfen worden).

a $\mathrm{B}+\mathrm{L}$ bezieht sich auf 3 Studien (408 Observationen) basierend auf dem Michigan Model of Production and Trade (Brown, Deardorff und Stern 2002, Brown, Deardorff und Stern 2003, Brown, Kiyota und Stern 2005), sowie 32 Observationen aus Lodefalk und Kinnman (2006), welche wesentlich größere Wohlfahrtsgewinne als das übrige Sample simulieren (siehe Diskussion im Text).

b Nur Observationen in Brown, Deardorff und Stern (2002); Brown, Deardorff und Stern (2003); Brown, Kiyota und Stern (2005) weisen diese Kombination von Modellcharakteristika auf, sodass die korrespondierende Dummy-Variable nicht in die Schätzung von Gleichung 3.14 aufgenommen wurde, sofern das "ohne B $+\mathrm{L}$ "Sample verwendet wird.

Quelle für Tabelle 2, 3, 4, 5, 6, 7 und 8: Eigene Berechnung.

\subsection{Diskussion}

Die vorgestellten Ergebnisse zeigen, dass eine einfache Meta-Regression unter Verwendung von Variablen, welche das Liberalisierungsexperiment, die Charakteristika der verwendeten Modelle und die verwendeten Daten abbilden, einen beträchtlichen Anteil der Variationen innerhalb simulierter Wohlfahrtsänderungen in einem Literatursample von Studien im Rahmen der Doha-Runde erklären kann. Die Ergebnisse ermöglichen quantitative Schätzungen von partiellen Einflüssen auf die Simulationsergebnisse, welche bisher nur qualitativ und ohne gleichzeitig die simultane Variation einer ganzen Reihe von Faktoren zu kontrollieren betrachtet werden konnten. Die geschätzten Koeffizienten sind überwiegend statistisch signifikant, haben plausible Vorzeichen und Größenordnungen und sind robust gegenüber Änderungen in der Spezifikation der Meta-Regression.

Diese Ergebnisse tragen zu einem besseren Verständnis darüber bei, wie Modellcharakteristika, Spezifizierung der simulierten Politikexperimente und die Datengrundlage Handelssimulationen beeinflussen. Die Ergebnisse dieser Analyse können darüber hinaus den Eindruck einer willkürlichen Streuung von Simulationsergebnissen reduzieren. Dieser Eindruck entsteht leicht, wenn für die simultane Variation mehrerer Faktoren in Simulationsmodellen nicht kontrolliert wird. Die hier vorgestellten ökonometrischen Schätzungen partieller Einflussgrößen können sowohl Modellierern als auch Nachfragern nach Modellergebnissen helfen, Simulationsexperimente zu vergleichen, zu evaluieren und 
wenigstens teilweise die Unterschiede zwischen unterschiedlichen Modellergebnissen $\mathrm{zu}$ erklären. Dies kann insbesondere für die am wenigsten entwickelten Länder sinnvoll sein, da diese häufig nicht über genug eigene Modellierungskapazitäten verfügen und es nicht sinnvoll wäre, spezialisiertes Personal in einem langwierigen Vergleich unterschiedlicher und einander möglicherweise widersprechender Simulationsergebnisse zu binden.

Die Tatsache, dass so viele möglicherweise informative Studien aus dem Datensatz eliminiert werden mussten, unterstreicht die Bedeutung einer transparenten Kommunikation hinsichtlich des experimentellen Aufbaus, mit welchen Simulationsergebnisse erstellt werden. $\mathrm{Zu}$ viele Studien dokumentieren nicht einmal die zugegebenermaßen verkürzten und komprimierten Informationen, welche verwendet wurden um die Meta-Analyse durchzuführen. Es kann davon ausgegangen werden, dass im Falle einheitlicher und vollständiger Dokumentation ein bedeutend größerer Anteil der Variation innerhalb simulierter Wohlfahrtseffekte im verwendeten Literatursample erklärt werden könnte. Darüber hinaus würde durch eine bessere Dokumentation die Kommunikation zwischen Modellierern und Anwendern von Modellergebnissen deutlich verbessert.

Es muss betont werden, dass die hier vorgestellten Ergebnissen nichts darüber sagen, welches Modell "das Beste" ist. Während die hier vorgestellte MetaAnalyse darauf hinweist, dass die Verwendung der Johansen-Macro-Closure im Gegensatz zur neoklassischen Macro-Closure systematisch höhere simulierte Wohlfahrtseffekte ausweist, kann dies keinerlei Hinweis darüber geben, welche dieser beiden Closures überhaupt benutzt werden sollte.

Insgesamt kann die vorliegende Analyse als ein Versuch gesehen werden, ein Meta-Response-Surface zu schätzen, wobei die verwendeten Daten aus publizierten oder anderweitig veröffentlichten Studien extrahiert wurden. Geplante, systematische Sensitivitätsanalyse anhand eines Versuchsplans mit einer Reihe zur Verfügung stehender Modelle und über Modellspezifikationen hinweg könnte eingesetzt werden, um den bestehenden Datensatz zu vervollständigen und um präzisere und detailliertere Schätzungen dieses Meta-Response-Surfaces zu ermöglichen. Wenn diese resultierende Meta-Analyse hinreichend präzise und übersichtlich in ihrer Struktur wäre, könnten predicted values sogar verwendet werden, um in einigen Fällen kostengünstige Alternativen zur tatsächlichen Modellierung, zumindest als Vorabschätzung zu liefern. Nichtsdestotrotz würden Anwender einer solchen, ergänzten Meta-Regression noch immer viele Entscheidungen treffen müssen, beispielsweise welche Macro-Closure gewählt werden soll, oder auch ob konstante bzw. steigende Skalenerträge unterstellt werden. Mit anderen Worten: Durch eine Meta-Analyse kann nicht vermieden werden, dass sich der Betrachter von Simulationsergebnissen mit den zu Grunde liegenden Annahmen auseinandersetzen muss, aber Meta-Analyse kann in 
diesem Zusammenhang den Blickwinkel auf die wichtigsten Modellcharakteristika lenken.

Die hier vorgestellte Meta-Analyse beleuchtet Faktoren, welche Simulationsergebnisse beeinflussen, die unter Verwendung aktueller, gängiger Modellierungspraktiken erstellt wurden. Das oben angesprochene Meta-ResponseSurface würde diese Praktiken ebenso enthalten. Kritiker könnten argumentieren, dass Meta-Analyse auf Basis dieser Informationen unter dem „Junk in- junk out" Problem leiden würde. Das bedeutet, diese Analyse beleuchtet, wie gegenwärtig verwendete Modelle funktionieren, aber dies ist nur insofern interessant, als man davon überzeugt ist, dass diese Modelle auch angemessene Repräsentationen wichtiger ökonomischer Phänomene (beispielsweise spekulativer Kapitalflüsse, IIT, Marktmacht, Skalenerträge, usw.) darstellen. Zukünftige Fortschritte im Bereich der Handelstheorie und Fortschritte hinsichtlich der Inkorporierung dieser Theorie in angewandten Gleichgewichtsmodellen werden notwendig sein, um diesen Bedenken zu begegnen und um immer bessere Modelle zu formulieren. 
Sebastian Hess - 978-3-631-75694-2

Downloaded from PubFactory at 01/11/2019 02:52:17AM

via free access 


\section{Erweiterung: Meta-Analyse und Response-Surface Design}

Meta-Analyse ermöglicht einen quantitativen Vergleich von Simulationsstudien, aber fehlende Werte und sich unterscheidende Modellspezifikationen machen es bei der Erfassung erklärender Variablen mitunter notwendig, auf wenig präzise, allgemeine Kategorien zurückzugreifen. Eine genaue Vorhersage von Simulationsergebnissen wird dadurch evtl. erschwert. Genauere ökonometrische Schätzungen können hingegen erzielt werden, indem eine Meta-Analyse anhand von Ergebnissen durchgeführt wird, die eigens zu diesem Zweck generiert wurden. Dieser Ansatz ist vergleichbar mit einer ökonometrischen Sensitivitätsanalyse über Modellansätze hinweg. Im Bereich ingenieurtechnischer Simulationsmodelle und auch im Bereich von Multi-Agentenmodellen existiert hierzu der Ansatz der sog. Response-Surface-Erstellung (synonym zu Meta-Modellierung), wobei jedoch stets die Robustheit der Aussagen eines einzelnen Modells untersucht wird; eine Kombination von Modellansätzen durch die Verwendung von Response-Surface-Design ist bisher insbesondere im Bereich von Handelsmodellen unüblich ${ }^{2}$ und soll nachfolgend eingeführt werden.

\subsection{Response-Surface und Meta-Modellierung}

Ein Response-Surface beschreibt den Zusammenhang zwischen einer abhängigen und mehreren erklärenden Variablen in einer Weise, die die Identifikation lokaler oder globaler Optima ermöglicht (Box und Wilson 1951). Im Zusammenhang mit der exogenen Parametrisierung angewandter Gleichgewichtsmodelle bedeutet dies, dass aufgrund der vielfältigen Interaktionseffekte innerhalb eines Simulationsmodells die in einem spezifischen Simulationsexperiment ausgewiesenen Wohlfahrts-, Einkommens-, Preis- und Handelsbilanz-Effekte eventuell (lokale) Extrempunkte verfehlen. Dies ist zunächst gleichzusetzen mit einer Sensitivitätsanalyse, welche unterstellt, dass bestimmte exogene Daten und Parameter einer stochastischen Verteilung unterliegen, ohne dass a priori ein eindeutiger Wert identifiziert werden kann, der alleine fur eine exogene Parametrisierung in Frage kommen würde, sprich, von welchem bekannt ist, dass er „der Richtige“ ist.

2 Es kann nicht ausgeschlossen werden, dass solche Applikationen existieren, aber bis dato konnten keine entsprechenden Publikationen identifiziert werden 
Sensitivitätsanalyse und Response-Surfaces versuchen daher, die Reaktionsweise der endogenen Variablen im Fokus einer simulierten Politikänderung innerhalb bestimmter Intervalle der exogenen Variablen abzubilden, um Aussagen darüber zu ermöglichen, ob ein bestimmtes Szenario eventuell durch relativ kleine Änderungen exogener Variablen eine relativ große Änderung der abhängigen Variable zeigt, was im Falle angewandter Gleichgewichtsmodelle meist als geringe Robustheit der Ergebnisse interpretiert wird.

Während Sensitivitätsanalysen meist von simulierten Experimenten extrapolieren und Durchschnitte oder Konfidenzintervalle errechnen, wird im Falle einer Response-Surface-Erstellung meist durch parametrische oder nicht-parametrische Schätzverfahren die durchschnittliche Reaktionsweise des Modells im Hinblick auf den funktionalen Zusammenhang zwischen endogener Variable als Funktion exogener Variablen geschätzt und somit ein Meta-Modell geschaffen (Banks 1998), welches typischerweise Polynome ersten oder zweiten Grades schätzt. Response-Surface-Erstellung kann im Falle angewandter Gleichgewichtsmodelle daher als eine allgemeine Form der Sensitivitätsanalyse betrachtet werden.

Solche ökonometrischen Meta-Modelle als Resultat einer Response-Surface Erstellung haben zunächst nichts mit Meta-Analysen gemein, da sie sich gewöhnlich auf nur ein Modell beziehen. Verwendet man jedoch generierte Daten aus mehreren Modellen, ergibt sich ein Meta-Datensatz, welcher ebenfalls ökonometrisch ausgewertet werden kann und wodurch sich eine synthetische MetaAnalyse ergibt.

Die Stärken einzelner Gleichgewichtsmodelle bzw. einzelner Modellansätze sind auf diese Weise kombinierbar, sofern sie innerhalb eines solchen MetaResponse-Surface zusammengefasst werden können: Viele partielle Gleichgewichtsmodelle bieten beispielsweise die Möglichkeit, komplexe Politikszenarien wie etwa die CAP mit ihren Markteingriffen, (teilweise) entkoppelten Direktzahlungen und Quoten detailliert abzubilden, was in CGE Modellen vergleichsweise großen technischen Aufwand erfordert (ein Modul zur Modellierung der CAP in GTAP findet sich bei Brockmeier 2003). Andererseits ist es kaum möglich, die Ergebnisse eines partiellen Gleichgewichtsmodells um eventuell bedeutsame gesamtwirtschaftliche Rückkopplungseffekte zu korri-gieren. Meta-Modellierung kann diese Kluft möglicherweise überbrücken. Ferner kann eine solche Meta-Modellierung evtl. eine Ausweisung robuster Korrekturfaktoren ermöglichen, welche beispielsweise angeben, um welchen Betrag sich die Ergebnisse eines Simulationsexperiments unter alternativen Modellansätzen (z.B. partielles vs. allgemeines Modell) ändern würden. Gegenüber literaturbasierten Meta-Analysen haben solche exemplarischen Meta-Modelle evtl. den Nachteil eingeschränkter Repräsentativität, was aber wiederum durch einen 
Vergleich mit den Ergebnissen einer literaturbasierten Meta-Analyse ausgeglichen werden kann.

Meta-Modellierung als Erweiterung und Ergänzung einer Meta-Analyse angewandter Gleichgewichtsmodelle verfolgt also drei Ziele:

- Überprüung der Abhängigkeit von Simulationsergebnissen hinsichtlich bestimmter, in der Literatur wiederholt auftretender Modellspezifikationen,

- Zusammenführung der Stärken unterschiedlicher Modellansätze in gemeinsamen ökonometrischen Meta-Modellen,

- Ausweisung robuster Korrekturfaktoren zur näherungsweisen Beurteilung von Simulationsergebnissen eines Modells im Kontext alternativer Modellannahmen, d.h. es wird untersucht, um welchen Betrag das Ergebnis einer simulierten Politikfolgenabschätzung verändert wäre, wenn anstelle des Modellansatzes A (z.B. CRTS) der Modellansatz B (z.B. IRTS) eingesetzt würde. Solche Erkenntnisse könnten eventuell auch genutzt werden, um fehlende Werte innerhalb einer literaturbasierten Meta-Analyse durch Imputation zu schließen.

\subsection{Applikation 1: Ein partiell-allgemeines Meta-Response- Surface}

Die Umsetzung dieser Ziele stößt insbesondere auf praktische Schwierigkeiten, welche aber unter bestimmten Umständen gelöst werden können, wie im Folgenden anhand eines vergleichsweise einfachen partiellen (PE) und eines einfachen allgemeinen Gleichgewichtsmodells (CGE) nachvollzogen werden soll:

Die Ergebnisse der Meta-Analyse von Hess und von Cramon-Taubadel (2007b) haben fur die Literatur im Rahmen der Doha-Runde die Rolle wichtiger Modellannahmen für Simulationsergebnisse quantifiziert. Einige dieser Modellannahmen haben einen besonders starken Einfluss auf die Simulationsergebnisse. Hiervon sollen exemplarisch zwei wichtige Grundannahmen betrachtet werden: der Unterschied zwischen partiellen und allgemeinen Gleichgewichtsmodellen sowie die Bedeutung der Armington-Annahme, gemessen anhand der Höhe der verwendeten Armington-Elastizitäten.

Als abhängige Variable werden simulierte Wohlfahrtsgewinne gewählt, wobei ebenso wie in der Meta-Analyse von Hess und von Cramon-Taubadel (2007b) genau unterschieden werden muss, wie "Wohlfahrtsänderung" innerhalb eines bestimmten Modells gemessen wird. Andernfalls ist keine sinnvolle Interpretation der gemessenen Regressionskoeffizienten möglich.

Die beiden hier verwendeten Modelle PE und CGE wurden von Francois und Hall (1997) entwickelt (http://www.intereconomics.com/handbook/ Models/ Index.htm), sind frei zugänglich und können bereits innerhalb eines Tabellen- 
kalkulationsprogramms mit Solver-Funktion gelöst werden. Das Modell PE existiert mit homogener sowie heterogener (Armington-) Präferenzspezifikation hinsichtlich der gehandelten Güter; darüber hinaus steht eine Version als einfaches allgemeines Gleichgewichtsmodell (nur mit Armington-Annahme) zur Verfugung. Die Armington-Version weist zwei konkurrierende Importlieferanten mit zwei voneinander unterschiedlichen Zollsätzen innerhalb von Land A auf. Die CGE-Armington-Version bettet dieses partielle Modell in einen einfachen gesamtwirtschaftlichen Kreislauf ein, in dem neben dem betrachteten Sektor ein zusätzlicher Numéraire-Sektor existiert. Summiert mit der inländischen Produktion in Sektor 1 bildet dieser Numéraire-Sektor das Bruttosozialprodukt (BSP) des Landes A. Anhand einer Ausgabenfunktion wird innerhalb des Modells die Menge des Gutes 1 bei Preis $p_{A, D}$ konsumiert.

Gleichung (5.1) zeigt, wie der Preis $p_{A, D}^{\prime \prime}$ für das inländisch nachgefragte Gut 1 durch die Armington-Annahme determiniert wird:

$$
p_{A D}^{\prime \prime}=\left[\omega_{D}^{\sigma}\left(p_{D}^{1-\sigma}\right)+\omega_{1}^{\sigma}\left(p_{1}^{1-\sigma}\right)+\omega_{2}^{\sigma}\left(p_{2}^{1-\sigma}\right)\right]^{\frac{1}{(1-\sigma)}}
$$

Dabei ist $p_{A, D}=$ Preis des Armington-Gutes, inländisch; $\omega=$ CES-Faktor; $\sigma=$ Armington-Elastizität, $p=$ Preis für Gut 1 in Land A aus Region $i$; $i=1,2$; vgl. Francois und Hall (1997) sowie die Originalversion des Modells.

Die Superskripte deuten an, dass für Preise und alle anderen Variablen drei zeitliche Stadien unterschieden werden müssen: $p=$ Preise im Modell unter Freihandel, $p^{\prime}=$ Preise nach Einführung der Ausgangszölle, $p^{\prime \prime}=$ Preise nach Durchführung des zu simulierenden Politikszenarios. Die ausgewiesenen Wohlfahrtseffekte beziehen sich folglich, sofern nicht anders angegeben, auf den Unterschied zwischen den Stadien $p^{\prime}$ und $p^{\prime \prime}$.

Für die hier durchgeführten Simulationsexperimente wurden in beiden Modellversionen die gleichen Beträge als Basisdaten festgelegt, d.h. die Ausgangsgröße aller drei Sektoren sowie die Gesamtausgaben der Konsumenten in Land 1 fur Produkt 1 bleiben konstant und spielen für das Experiment keine Rolle. Wählt man für diese Basisdaten andere Größenordnungen, ändern sich die absoluten Wohlfahrtseffekte in Abbildung 8.

Das verwendete CGE Modell weist ursprünglich Äquivalente Variation (EV) als Wohlfahrtsmaß aus. Dieses Maß wird hier ignoriert, da es im PE Modell nicht vorkommt. Stattdessen wird auch im CGE Modell für Sektor 1 in Land 1 die partielle Wohlfahrtsänderung wie in Gleichung (5.4) und (5.5) als Resultat des Handels mit beiden Importkonkurrenten erhoben, was die ursprüngliche Modellspezifikation geringfügig verändert. 
Diese Betrachtung partieller Wohlfahrtseffekte innerhalb eines allgemeinen Gleichgewichtsmodells ist zulässig, da für den Ausgabenanteil des einzigen konsumierten Gutes kontrolliert wird (Mas-Colell, Whinston und Green 1995).

Nachdem auf diese Weise Basisdaten und Wohlfahrtsmessung in beiden Modellen aneinander angepasst wurden, verbleibt lediglich die Ausgestaltung der Ausgabenfunktion als Unterschied zwischen dem PE und dem CGE Modell:

$$
\begin{gathered}
E_{D}^{\prime \prime}=E^{\prime}\left(p_{A D}^{\prime \prime}\right)^{\left(-\eta_{D}+1\right)} \\
E_{D}^{\prime \prime}=\left\langle\left[Q_{N}^{\prime}+Q_{D}^{\prime}\left(p_{D}^{\prime \prime}{ }^{\varepsilon+1}\right)+M_{1}\left(\frac{p_{2}^{\prime \prime}}{1+t_{2}^{\prime \prime}}\right)+M_{2}\left(\frac{p_{3}^{\prime \prime}}{1+t_{3}^{\prime \prime}}\right)\right]\right) \theta_{D}
\end{gathered}
$$

Gleichung (5.2) repräsentiert die Ausgabenfunktion im PE Modell. $E_{D}$ sind dabei die Gesamtausgaben in Land A (D=Domestic), $Q_{M}$ und $Q_{D}$ sind Inlandsproduktion im Numeraire Sektor sowie im betrachteten Sektor 1; $M_{I}$ und $M_{2}$ sind die Importmengen in A aus Importland 1 (Land B) und Importland 2 (Land C). Durch $t$ werden die jeweiligen bilateralen Importzölle bezeichnet. Es ist unschwer zu erkennen, dass diese Funktion wesentlich von der Nachfrageelastizität $\eta^{\mathrm{D}}$ dominiert wird. Die Ausgabenfunktion im CGE Modell in Gleichung (5.3) hingegen weist keine Nachfrageelastizität auf und bestimmt die Ausgaben für Gut 1 vielmehr über den Ausgabenanteil $\theta_{D}$ dieses Gutes 1 in Land A.

\subsubsection{Experimentelles Design}

Mit Hilfe einer Visual Basic (VBA (B) Routine wurden die in Tabelle 9 genannten exogenen Modellparameter in den angegebenen Bereichen durch Generierung von Pseudo-Zufallszahlen variiert, um Zufallsszenarien zu erzeugen. Dazu wurde jeder generierte Wert von der VBA-Routine zunächst in eine Datenbank geschrieben und nach Lösung des Szenarios wurden diesen Ausgangswerten innerhalb der Datenbank die entsprechenden Simulationsergebnisse zugeordnet. Da Rechnerkapazität bei keinem der beiden Modelle einen begrenzenden Faktor darstellt, wurde für jede der beiden Modellvarianten die Zahl der zu simulierenden Szenarios auf 4000 festgelegt (ca. $3 \mathrm{~h}$ Simulationsdauer). Im Falle des PE Modells konnte der Solver für alle 4000 Szenarien eine Lösung finden; für das CGE war dies bei $23 / 4000=0.6 \%$ der Szenarien nicht der Fall.

Als Politikszenario wurde eine unilaterale Veränderung des Importzolls in Land A für Importe aus zwei konkurrierenden Anbieterländern $B$ und $C$ gewählt und es wird untersucht, wie sich die Variable „Wohlfahrtsänderung" als Funktion dieser Politikänderung verhält. Gleichzeitig werden weitere exogene Mo- 
dellparameter innerhalb bestimmter Intervalle für jedes Szenario zufällig gewählt (siehe Tabelle 9).

Der hier vorgestellte Ansatz hebt sich von vergleichbaren Ansätzen zur empirischen Validierung angewandter Gleichgewichtsmodelle in folgender Weise ab: Da exogene Parameter nur selten zweifelsfrei und mit der eigentlich notwendigen Präzision bekannt sind, wird hier unterstellt, dass Schlüsselparameter innerhalb eines plausiblen Intervalls jeden möglichen Wert annehmen können. Eine Alternative zu dieser Annahme bestünde in der Durchfuhrung einer Meta-Analyse publizierter Nachfrageelastizitäten, um die entsprechenden Werte möglichst präzise und über viele Studien hinweg zu ermitteln (z.B. Kremers, Nijkamp und Rietveld 2000), was bei der Vielzahl an Parametern innerhalb angewandter Gleichgewichtsmodelle insgesamt sehr zeitaufwendig sein würde.

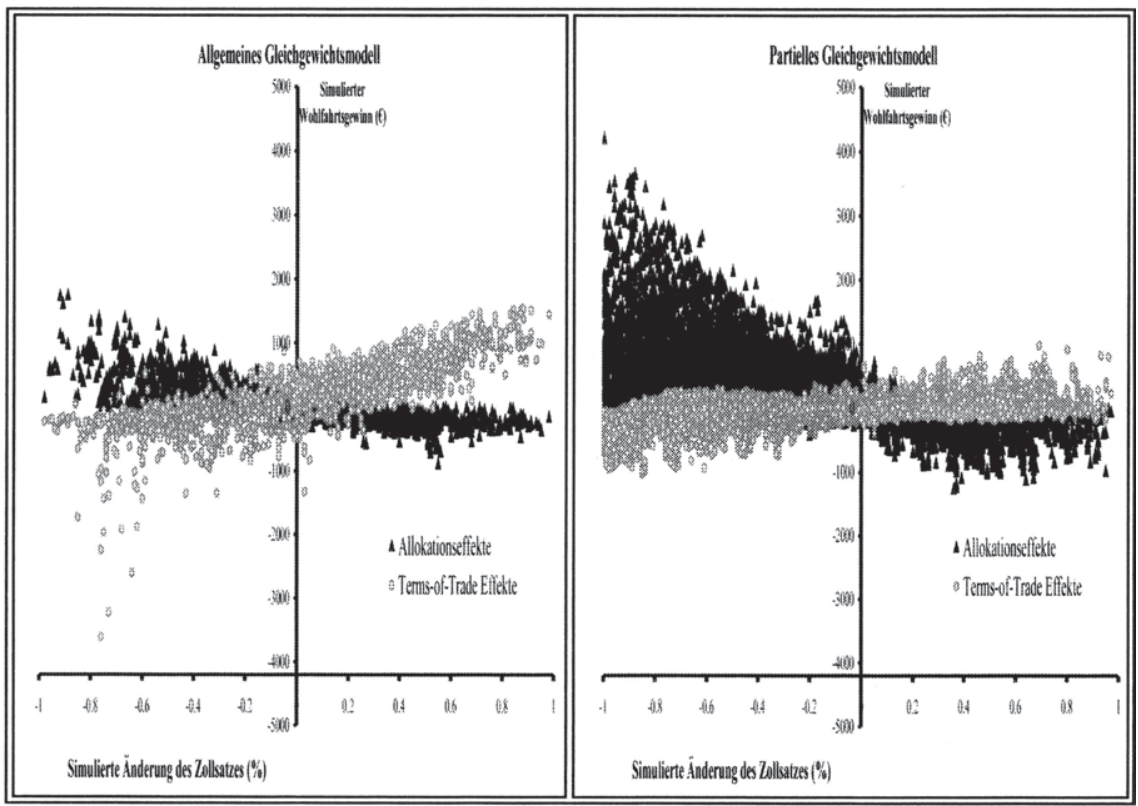

Abbildung 8: Simulierte Wohlfahrtseffekte für CGE (links) und PE (rechts).

Quelle: Eigene Berechnungen.

Abbildung 8 zeigt die simulierten Wohlfahrtsänderungen, aufgeteilt in allokative Effekte und ToT-Effekte gemäß der Gleichungen (5.4) und (5.5). In diesen Gleichungen bezeichnet $r=1,2$ die Zahl der beiden Handelspartner, aus welchen 
Land A Importe bezieht. Die übrigen Variablen folgen den Definitionen für Gleichung (5.1), Gleichung (5.2) und Gleichung (5.3).

$$
\begin{gathered}
\Delta \operatorname{Harbg}_{D}=M_{r}^{\prime}\left(1+\Delta M_{r}^{\prime}\right)-\left(\frac{\omega_{r}}{p_{r}^{\prime}}\right)^{\sigma}\left(E_{D}^{\prime}\left(p_{A D}^{\prime(\sigma-1)}\right)\right)\left[p_{r}^{\prime}-p_{r}^{\prime}\right] \frac{1}{2} \\
\Delta T_{o} T_{D}=\left[p_{r}^{\prime}-\left(\frac{p_{r}^{\prime}}{1+t_{r}^{\prime}}\right)\right]\left(1+\Delta M_{r}^{\prime}\right) Q_{r}
\end{gathered}
$$

Abbildung 8 bestätigt die Einschätzung, dass das hier verwendete CGE Modell die Ergebnisse c. p. „dämpft“ (vgl. z.B. Banse und Tangermann 1996) und steht evtl. im Widerspruch zu jüngeren Untersuchungen anhand des GTAP-Modells (Gohin und Moschinni 2005), welche keine deutlichen Unterschiede zwischen einer PE Closure im Vergleich zu einer General-Equilibrium-Closure innerhalb von GTAP finden, wobei die betrachtete Wohlfahrtsänderung - anders als im vorliegenden Experiment - Äquivalente Variation (EV) ist. Hieran wird deutlich, dass vermeintliche „Binsenweisheiten“ im Einzelfall eines jeden Modells der empirischen Überprüfung bedürfen.

\begin{tabular}{|c|c|c|c|}
\hline $\begin{array}{l}\text { Basisdaten der Modelle PE } \\
\text { und CGE, die in allen Sze- } \\
\text { narien konstant bleiben }\end{array}$ & $\begin{array}{l}\text { Wert } \\
\text { (willkürlich ge- } \\
\text { wăhlt, Geld- } \\
\text { einheiten) }\end{array}$ & $\begin{array}{l}\text { Exogene Variablen der Model- } \\
\text { le; Parametrisierung je Simula- } \\
\text { tion zufallig innerhalb der In- } \\
\text { tervalle: }\end{array}$ & $\begin{array}{l}\text { Spezifiziertes Intervall für } \\
\text { Modellparameter k }\end{array}$ \\
\hline $\begin{array}{l}\text { Produktion Land A, Sektor } \\
1\end{array}$ & 1000 & $\Delta$ Zoll Land B & $0.01<k<0.99$ \\
\hline $\begin{array}{l}\text { Importe aus Land B, Sek- } \\
\text { tor } 1\end{array}$ & 2000 & $\Delta$ Zoll Land C & $0.01<k<0.99$ \\
\hline $\begin{array}{l}\text { Importe aus Land C, Sek- } \\
\text { tor } 1\end{array}$ & $5000,-$ & $\sigma$ (Armington- El.) & $0.01<k<50$ \\
\hline $\begin{array}{l}\text { Gesamtausgaben fulr Gut } 1 \\
\text { aus Sektor } 1 \text { in Land A }\end{array}$ & $8000,-$ & $\varepsilon_{S}($ Land A $)$ & $0.01<k<10$ \\
\hline \multirow{4}{*}{\multicolumn{2}{|c|}{$\begin{array}{l}\text { Solver-Einstellungen: } \\
\text { Iterationen: } 1000 \\
\text { Konvergenz: } 10^{-9} \\
\text { Genauigkeit: } 10^{-9} \text {, Toleranz: } 10^{-6} \\
\text { automatisch skalieren, Newton }\end{array}$}} & $\varepsilon_{M}($ Land B $)$ & $0.01<k<10$ \\
\hline & & $\varepsilon_{M}($ Land $C)$ & $0.01<k<10$ \\
\hline & & GDP Land $A$ (nur CGE) & $8000<k<8000000$ \\
\hline & & $\eta_{D}$ in Land $A$ (nur PE) & $0.01<k<3$ \\
\hline
\end{tabular}

Tabelle 9: Versuchsaufbau für ein partiell-allgemeines Meta-Modell.

Quelle: Eigene Zusammenstellung.

Im hier dargestellten Fall kann der ,dämpfende“ Einfluss von CGE relativ zu PE eindeutig auf die unterschiedliche Spezifikation der Gleichungen (5.2) und (5.3) zurückgeführt werden. Im Falle komplexerer Modelle wie GTAP sind ggf. meh- 
rere Gleichungen am Zustandekommen des Unterschiedes zwischen PE und CGE beteiligt. Der letztendlich ausgewiesene Effekt der Änderung einer endogenen Variable muss folglich keineswegs immer die gleiche oder auch nur eine über Modelle hinweg ähnliche Größenordnung aufweisen (vgl. Gohin und Moschinni 2005).

Abbildung 8 lässt erkennen, dass die simulierten Wohlfahrtseffekte bei größeren Liberalisierungsschritten mit einer stetig zunehmenden Varianz streuen. Vergleicht man Abbildung 8 mit Abbildung 2, so lassen sich durchaus ähnliche Effekte konstatieren: Auch in Abbildung 2 scheint die Varianz innerhalb von publizierten Ergebnissen mit größeren Liberalisierungsschritten zuzunehmen (vgl. in Abbildung 2 „Harbinson- Vorschlag“ im Vergleich zu „Abschaffung aller Zölle und Protektion weltweit“).

\subsubsection{Parametrische und Nicht-Parametrische Meta-Modellierung}

Für die $n=7$ gleichzeitig variierten Parameter ergeben sich bei Berücksichtigung aller Interaktionsterme als Summe aller $k=1,2, \ldots, 7$ Gruppierungsmöglichkeiten insgesamt

$$
\sum_{k=1}^{7} \frac{n !}{n !-k !}=127
$$

Addiert man zu diesen 127 Koeffizienten noch den Achsenabschnitt, so ergeben sich $128 \mathrm{zu}$ schätzende Koeffizienten (die alternativ auch als $2^{7}=128$ berechnet werden können), was deutlich macht, dass selbst bei einem vergleichsweise einfachen Response-Surface wie in diesem Fall ein Kompromiss zwischen Vereinfachung des ökonometrischen Meta-Modells und Qualität der Schätzung erstrebenswert ist (Kleijnen et al. 2005). Schätzt man jedoch alle Interaktionseffekte, lassen sich bei gleichzeitiger Berücksichtigung quadratischer Terme nahezu 100 Prozent der Varianz in der abhängigen Variable „Harberger Dreiecke“ erklären; für ToT-Effekte fällt dieser Wert mit ca. 90 Prozent geringfügig niedriger aus, d.h. es müssten evtl. andere Funktionsverläufe zusätzlich zu den Interaktionstermen berücksichtigt werden, sofern ein höheres $R^{2}$ angestrebt wird.

Im vorliegenden Experiment wurden zunächst nicht-parametrische Regression Splines (Maindonald und Brown 2003) angewendet, um für jeden exogenen Parameter den Funktionsverlauf $\mathrm{zu}$ determinieren. Durch lineare Regressionsmodelle können jedoch im Gegensatz zu nicht-parametrischen Schätzern marginale Effekte ausgewiesen werden, was eine Interpretation des Meta-Modells aufgrund der Ausweisung marginaler Effekte wesentlich erleichtert. Im vorliegenden Fall wurde bei der Schätzung des Meta-Modells eine besonders komprimierte Darstellung gewählt; unabhängig von den tatsächlichen, nicht-parametrisch geschätzten Funktionsverläufen wurde ledig- 
lich für die simulierten Zolländerungen ein quadratischer Term zugelassen, um zu berücksichtigen, dass allokative Wohlfahrtsverluste proportional mit dem Quadrat des Zollsatzes steigen (Nicholson 1998).

Allokative Effekte und ToT-Effekte können entweder als Summe innerhalb einer Regression oder separat betrachtet werden. Es muss aber berücksichtigt werden, dass die ToT-Komponente im Fall der Annahme eines „kleinen“ Landes oft ignoriert wird, weshalb eine getrennte Betrachtung von ToT- und allokativen Effekten im Sinne einer Meta-Modellierung sinnvoll ist. Die Verwendung einer Dummy-Variable hingegen erscheint nicht sinnvoll, da Abbildung 8 bereits graphisch veranschaulicht, dass allokative Effekte und ToT-Effekte weitgehend gegenläufige Vorzeichen aufweisen. Mit anderen Worten: Die getrennte Meta-Modellierung von allokativen- und ToT-Effekten beinhaltet wahrscheinlich aufschlussreiche Informationen über die Zusammensetzung und Entstehung der gesamten simulierten Wohlfahrtswirkung. Nichtsdestotrotz kann die Verwendung einer Dummy-Variablen innerhalb einer Pooled Regression hinsichtlich ihrer Erklärungskraft gegenüber zwei getrennten Regressionen überprüft werden.

Tabelle 9 verdeutlicht noch einmal, was schon in Gleichung (5.2) und (5.3) offenkundig wurde und ein typisches Problem bei Vergleich und Kombination von angewandten Gleichgewichtsmodellen darstellt: PE weist keine explizite Repräsentation von BSP und damit keine Aussagen über den Einkommensanteil $\theta_{D}$ von Sektor 1 in Land A auf, während CGE ohne explizite Repräsentation der Nachfrageelastizität auskommt. Tabelle 10 präsentiert daher zwei Seemingly Unrelated (SUR) Modelle (Zellner 1962). SUR 1 schätzt jeweils für CGE und für PE die durchschnittlich simulierten Allokationseffekte („Harberger“); SUR 2 führt die gleiche Schätzung mit ToT als abhängiger Variable durch. Die Ergebnisse von SUR-Modellen sind identisch mit separaten OLS-Schätzungen, sofern die Fehlerterme der simultan geschätzten Gleichungen statistisch unabhängig voneinander sind, was im Fall von zwei einander sehr ähnlichen Simulationsmodellen keineswegs zutreffen muss.

Ein SUR-Modell ermöglicht die simultane Schätzung beider unterschiedlicher Regressionsgleichungen, nämlich der für PE und CGE. Besteht das Ziel jedoch nicht in der getrennten Ausweisung der Effekte fü PE und CGE, sondern in einer Kombination beider Modelle mit dem Ziel der Ausweisung durchschnittlich zu erwartender Simulationsergebnisse, müssen entweder beide Variablen, BSP und $\eta_{D}$, aus der Regression entfernt werden, was evtl. verzerrte Schätzergebnisse zur Folge hätte oder die jeweils fehlenden Werte müssen durch Imputation ersetzt werden.

Ein einfach anzuwendendes und weit verbreitetes Imputationsverfahren ist die Ersetzung fehlender Werte durch den Mittelwert der jeweiligen Variable; eine aus algebraischer Sicht identische Variante dieser Methode besteht in der 
Ersetzung fehlender Werte durch 0 und Einführung einer zusätzlichen DummyVariable mit dem Wert „1“ im Falle eines fehlenden Wertes (Greene 2003).

Tabelle 10: Zwei Meta-SUR für Wohlfahrtsänderung und ToT. Ein vertikaler Vergleich der Koeffizienten innerhalb der Tabelle zeigt Unterschiede zwischen PE und CGE; ein horizontaler Vergleich zeigt die Unterschiede zwischen ,Harberger “ Dreiecken und ToTEffekten des jeweiligen Modells.

\begin{tabular}{|c|c|c|c|c|c|c|c|c|c|c|}
\hline & \multicolumn{5}{|c|}{$\begin{array}{l}\text { SUR 1: Allokativer Wohlfahrtseffekt } \\
\text { ("Harberger Dreieck") }\end{array}$} & \multicolumn{5}{|c|}{ SUR 2: Terms-of-Trade Effekt } \\
\hline & \multicolumn{5}{|c|}{ Allgemeines Gleichgewichtsmodell (CGE) } & \multicolumn{5}{|c|}{ Allgemeines Gleichgewichtsmodell (CGE) } \\
\hline Variable & \multirow{2}{*}{$\begin{array}{r}\text { Koeff. } \\
-511,08\end{array}$} & Std. Fehl. & \multicolumn{2}{|c|}{ t-Wert } & $\operatorname{Pr}(>|t|)$ & Koeff. & Std. Fehl. & \multicolumn{2}{|c|}{ t-Wert } & \multirow{2}{*}{$\begin{array}{r}\operatorname{Pr}(>|t|) \\
0,00\end{array}$} \\
\hline Achsenabschn. & & \multirow{2}{*}{$\begin{array}{l}16,85 \\
41,27\end{array}$} & \multicolumn{2}{|c|}{$-30,33$} & 0,00 & \multirow{2}{*}{$\begin{array}{l}636,96 \\
327,02\end{array}$} & 34,62 & \multicolumn{2}{|r|}{18,40} & \\
\hline$\Delta$ Zoll Land B & $-517,01$ & & \multirow{2}{*}{\multicolumn{2}{|c|}{$\begin{array}{l}-12,53 \\
-29,15\end{array}$}} & 0,00 & & 84,81 & \multicolumn{2}{|r|}{3,86} & 0,00 \\
\hline$\Delta$ Zoll Land C & $-1207,90$ & 41,44 & & & 0,00 & 1018,71 & 85,16 & \multicolumn{2}{|r|}{11,96} & 0,00 \\
\hline$(\Delta \text { Zoll } B)^{2}$ & $-36,46$ & 39,91 & \multicolumn{2}{|c|}{$-0,91$} & 0,36 & 193,91 & 82,02 & & 2,36 & 0,02 \\
\hline$(\triangle \mathrm{Zoll} \mathrm{C})^{2}$ & $-51,5$ & 6,35 & & 3,12 & 0,00 & -9 & 13,05 & & $-0,70$ & 0,48 \\
\hline$\sigma($ Arm-El.) & 3,4 & 0,20 & & 7,11 & 0,00 & & 0,42 & & 0,41 & 0,68 \\
\hline$\varepsilon_{S}($ Land A) & 8,9 & 1,02 & & 3,79 & 0,00 & -3 & 2,10 & & $-1,75$ & 0,08 \\
\hline$\varepsilon_{M}($ Land $B)$ & 31,3 & 1,04 & &, 05 & 0,00 & & 2,14 & & 0,60 & 0,55 \\
\hline$\varepsilon_{M}($ Land $C)$ & 55,6 & 1,02 & & 1,46 & 0,00 & 11 , & 2,10 & & 5,32 & 0,00 \\
\hline GDP/1000 & 0,1 & 0,01 & & 3,93 & 0,00 & -0, & 0,03 & & $-1,51$ & 0,13 \\
\hline & Koef & \begin{tabular}{l|l} 
if. & Std. Fehl.
\end{tabular} & $t-V$ & Vert & $\operatorname{Pr}(>|t|)$ & Koe & \begin{tabular}{l|l} 
eff. & Std. Fehl.
\end{tabular} & & Wert & $\operatorname{Pr}(>|t|)$ \\
\hline Achs & $-1945,5$ & 44,28 & & 394 & 0,00 & $-391, \mathrm{C}$ & 26,93 & & $-14,52$ & 0,00 \\
\hline $\mathrm{d} \mathrm{B}$ & $-677,0$ & 107,99 & & 6,27 & 0,00 & -741, & 65,69 & & $-11,29$ & 0,00 \\
\hline$\triangle$ Zoll L & $-1807,6$ & 109,34 & & 5,53 & 0,00 & -907, & 66,51 & & $-13,64$ & 0,00 \\
\hline$(\Delta \text { Zoll B })^{2}$ & 47,1 & 105,07 & &, 45 & 0,65 & $-994,8$ & 63,91 & & $-15,57$ & 0,00 \\
\hline$(\triangle \text { Zoll C })^{2}$ & $-64,7$ & 17,06 & &, 79 & 0,00 & -220 & 10,37 & & $-21,26$ & 0,00 \\
\hline$\sigma($ Arm. -El.) & 4,1 & 0,53 & & 7,83 & 0,00 & -2, & 0,32 & & $-6,38$ & 0,00 \\
\hline$\varepsilon_{S}($ Land $A)$ & 5,2 & 2,65 & &, 98 & 0,05 & -4, & 1,61 & & $-3,07$ & 0,00 \\
\hline$\varepsilon_{M}($ Land $B)$ & 61,3 & 2,62 & &, 38 & 0,00 & & 1,59 & & 2,38 & 0,02 \\
\hline$\varepsilon_{M}($ Land $C)$ & 115,6 & 2,64 & &, 74 & 0,00 & 29, & 1,61 & & 18,12 & 0,00 \\
\hline$\eta_{D}$ & 662,5 & 8,74 & & 8,80 & 0,00 & -34 & 5,32 & & $-6,41$ & 0,00 \\
\hline Gleichung & Df & MSE & $\begin{array}{l}\text { Res.- } \\
\text { MSE }\end{array}$ & $\mathbf{R} 2$ & $\begin{array}{r}\text { R2 } \\
\text { Adj } \\
\end{array}$ & Df & MSE & $\begin{array}{l}\text { Res.- } \\
\text { MSE }\end{array}$ & $\mathbf{R} 2$ & R2 Adj \\
\hline CGE & 3965 & 34062,4 & 184,6 & 0,779 & \begin{tabular}{|l|l|}
9 & 0,779 \\
\end{tabular} & 3965 & 571192594 & 379,5 & \begin{tabular}{|l|l|}
5 & 0,406 \\
\end{tabular} & 0,404 \\
\hline PE & 3965 & 902651302 & 477,1 & 0,746 & \begin{tabular}{l|l|}
6 & 0,745 \\
\end{tabular} & 3965 & 84352,4 & 290,4 & \begin{tabular}{|l|l|}
4 & 0,356 \\
\end{tabular} & 0,354 \\
\hline System & & & & 0,751 & & & & & 0,388 & \\
\hline
\end{tabular}

Quelle: Eigene Berechnung.

Little (1992) diskutiert und vergleicht weitere Imputationsverfahren empirisch miteinander. Im Falle des hier vorgestellten CGE-PE-Meta-Modells wurde aus den in Little (1992) vorgestellten Verfahren als mögliche Alternative zur Mittelwertmethode auch der auf dem Maximum-Likelihood-Schätzer beruhende EMAlgorithmus (Dempster, Laird und Rubin 1977) gewählt, was im Falle des ökonometrischen Meta- Modells in Tabelle 11 jedoch zu nahezu identischen Schätzergebnissen fuhrte. 
Hieraus kann geschlossen werden, dass die Verwendung des ExpectationMaximization (EM) Algorithmus im Falle des vorliegenden Meta-Modells keinen Vorteil gegenüber Imputation durch Mittelwerte bedeutet. In Tabelle 11 sind daher nur die Ergebnisse der Mittelwertimputation ausgewiesen.

Tabelle 11 präsentiert alternativ zu Tabelle 10 ein partiell-allgemeines MetaModell (OLS Schätzer mit robusten Standardfehlern). Die Hypothese der Homoskedastizität wurde durch den Breusch-Pagan Test abgelehnt. Besonders für die Allokationseffekte zeigt sich, dass durch das Regressionsmodell eine hinreichend genaue Approximation von PE und CGE innerhalb der in Tabelle 9 definierten Grenzen möglich ist. Das partiell-allgemeine Meta-Modell kann als eigenständiges Modell interpretiert werden, welches Vorhersagen über simulierte Wohlfahrtsänderungen als Folge von Zollsatzänderungen erlaubt, nachdem Annahmen über $\mathrm{zu}$ erwartende Bandbreiten wichtiger exogener Parameter getroffen wurden.

Tabelle 11: PE-CGE-OLS-Meta-Modell mit robusten Standardfehlern. Fehlende Werte in $G D P$ und $\eta D$ wurden durch Mittelwert dieser Variablen ersetzt.

\begin{tabular}{|c|c|c|c|c|c|c|c|c|}
\hline & \multicolumn{4}{|c|}{$\begin{array}{c}\text { Allokativer Wohlfahrtseffekt } \\
\text { ("Harberger Dreieck") }\end{array}$} & \multicolumn{4}{|c|}{ Terms-of-Trade Effekte } \\
\hline Variable & Koeff. & Std. Fehl. & t-Wert & $\operatorname{Pr}(>|t|)$ & Koeff. & Std. Fehl. & t-Wert & $\operatorname{Pr}(>|t|)$ \\
\hline Achsenabschn. & $-1666,097$ & 29,689 & $-56,118$ & 0,000 & 325,637 & 30,545 & 10,661 & 0,000 \\
\hline$\Delta$ Zoll Land B & $-588,933$ & 63,339 & $-9,298$ & 0,000 & $-184,678$ & 61,573 & $-2,999$ & 0,003 \\
\hline$\triangle$ Zoll Land C & $-1525,993$ & 65,101 & $-23,440$ & 0,000 & 49,138 & 69,456 & 0,708 & 0,479 \\
\hline$(\triangle \mathrm{Zoll} \mathrm{B})^{2}$ & 12,173 & 61,886 & 0,197 & 0,844 & $-376,047$ & 62,266 & $-6,039$ & 0,000 \\
\hline$(\Delta \text { Zoll C })^{2}$ & $-61,798$ & 10,170 & $-6,077$ & 0,000 & $-725,564$ & 68,117 & $-10,652$ & 0,000 \\
\hline$\sigma($ Arm.-El.) & 3,805 & 0,297 & 12,818 & 0,000 & $-0,881$ & 0,276 & $-3,193$ & 0,001 \\
\hline$\varepsilon_{S}($ Land $A)$ & 7,104 & 1,459 & 4,869 & 0,000 & $-4,721$ & 1,425 & $-3,314$ & 0,001 \\
\hline$\varepsilon_{M}($ Land $B)$ & 46,404 & 1,642 & 28,2 & 0,000 & 2,321 & 1,519 & 1,529 & 0,126 \\
\hline$\varepsilon_{M}($ Land $C)$ & 85,127 & 1,783 & 47,742 & 0,000 & 19,841 & 1,763 & 11,252 & 0,000 \\
\hline GDP/1000 & 0,129 & 0,017 & 7,767 & 0,000 & $-0,035$ & 0,029 & $-1,224$ & 0,221 \\
\hline$\eta_{D}$ & 662,507 & 11,275 & 58,757 & 0,000 & $-35,031$ & 5,829 & $-6,009$ & 0,000 \\
\hline \multirow[t]{3}{*}{ PE dummy } & 87,909 & 7,043 & 12,482 & 0,000 & $-342,620$ & 8,215 & $-41,706$ & 0,000 \\
\hline & \multicolumn{4}{|c|}{$\begin{array}{l}\text { Standardfehler der Residuen: } \\
382,9 \text { bei } 7938 \text { Freiheitsgraden }\end{array}$} & \multicolumn{4}{|c|}{$\begin{array}{l}\text { Standardfehler der Residuen: } \\
355,9 \text { bei } 7938 \text { Freiheitsgraden }\end{array}$} \\
\hline & \multicolumn{2}{|c|}{ Adjusted R- Squared: } & \multicolumn{2}{|c|}{0,737} & Adjusted $\mathrm{R}$ & -Squared: & \multicolumn{2}{|c|}{0,417} \\
\hline
\end{tabular}

Quelle: Eigene Berechnung.

Aufgrund der durch Abbildung 8 auch intuitiv verdeutlichten großen Abhängigkeit spezifischer Simulationsergebnisse von diesen Annahmen kann aber auch argumentiert werden, dass durch die in Tabelle 11 vorhergesagten Werte im Sinne einer modellübergreifenden Sensitivitätsanalyse eine transparente und robuste Möglichkeit zur Betrachtung von Simulationsergebnissen beider Modelle entstanden ist, da die numerischen Effekte eines jeden Einzelszenarios unter der Annahme nicht exakt spezifizierbarer Schlüsselparameter in den Kontext ihres stochastischen Erwartungswerts eingeordnet werden. 
Insgesamt zeigt sich anhand von Response-Surface Applikation 1, dass bereits zwei sehr einfache Modelle, CGE und PE, auf bestimmte Schwierigkeiten verweisen, welche bei der Erstellung von Response-Surfaces entstehen. Diese Schwierigkeiten liegen insbesondere in der eingeschränkten theoretischen Vergleichbarkeit unterschiedlicher Modellkomponenten, wodurch die Schätzung innerhalb einer Regression erschwert wird.

Im vorliegenden Fall war jedoch eine Imputation der jeweils fehlenden Werte möglich, sodass ein besonders übersichtliches lineares Meta-Modell geschätzt werden konnte, welches PE und CGE vereint. Für komplexere ResponseSurface Applikationen wäre es wahrscheinlich entsprechend schwieriger, fehlende Werte stets durch Imputationsverfahren generieren zu können. Ferner könnte ein lineares Meta-Modell grundsätzlich ungeeignet sein, um komplexe Modelloberflächen abzubilden.

\subsection{Applikation 2: Meta-Modellierung bilateraler Zoll- präferenzen im Welt-Zuckermarkt}

Im Rahmen des DFG-Forschungsprojektes „Evaluation angewandter Gleichgewichtsmodelle des Agrarsektors: Quantifikation systematischer Zusammenhänge zwischen Modellspezifikationen und Prognoseergebnissen" wurde neben der vorliegenden Dissertationsschrift auch eine Bachelorarbeit (Fahlbusch 2007) angefertigt. Ziel der Vergabe dieser Arbeit war es, einen Studenten durch die Entwicklung eines zweifaktoriellen Response-Surfaces an die Anwendung von Handelsmodellen heranzuführen. Dabei wurde die starke Abhängigkeit der Simulationsergebnisse von getroffenen Modellannahmen bereits in die Aufgabenstellung einbezogen. Ein partielles, grafisches 1-Sektor-2-Länder-Modell mit Kalkulation der partiellen Wohlfahrtseffekte sowie Berücksichtigung der ToT wurde als Referenz gewählt und das $\mathrm{zu}$ 1-Sektor-12-Regionen aggregierte partielle Gleichgewichtsmodell für den Welt-Zuckermarkt GSIM (mit Armington-Annahme) sollte durch Variation der präferentiellen Importzölle als einzigem Politikparameter sowie durch simultane Variation der ArmingtonElastizitäten in einer linearen Regression als einfaches Meta-Modell abgebildet werden. Dabei sollte das grafische Modell als theoretische Referenz dienen, um die anhand des GSIM-Modells errechneten Ergebnisse nachzuvollziehen und Abweichungen von diesem Referenzmodell identifizieren zu können. Das GSIM-Modell wurde von Francois und Hall (2003) entwickelt, ist frei zugänglich und wird gelegentlich zur Politikfolgenabschätzung eingesetzt. Während viele globale Doha-Szenarien auf MFN- Zollreduktionen beschränkt sind, bietet das GSIM-Modell eine detaillierte Abbildung bilateraler Handels-ströme und 
präferentieller bilateraler Zollsätze. Die Ergebnisse dieser Arbeit von Fahlbusch (2007) lassen sich wie folgt zusammenfassen:

Das GSIM-Modell bildet insbesondere für jedes Land die präferentiellen tarifären Handelsbarrieren detailliert ab, sodass die Verwendung eines MFNZolles im Rahmen einer Meta-Modellierung nicht aussagekräftig wäre, denn eine simultane Variation aller bilateralen Importzölle enthält $12^{2}=144$ bilaterale Zölle, weshalb theoretisch 144 Koeffizienten innerhalb eines Meta-Modells geschätzt werden müssten. Die Arbeit von Fahlbusch (2007) versucht daher, die Ergebnisse von Anderson und van Wincoop (2001) empirisch innerhalb des GSIM-Modells zu reproduzieren, indem insbesondere die Rolle großer Importeure versus großer Exporteure für Zucker unter Einfluss der Armington-Annahme beleuchtet wird. Es wird die Hypothese getestet, ob die relative Höhe der Handelsbarrieren zwischen zwei Ländern relativ zur Höhe der durchschnittlichen weltweiten Handelsbarrieren im Zuckersektor die Höhe der aus Liberalisierung resultierenden Wohlfahrtseffekte entscheidend determinieren. Die Untersuchungen anhand eines einfachen Response-Surface-Designs bestätigen dies empirisch für die Rolle eines großen Exporteurs, aber sind für die Rolle eines großen Importeurs nicht signifikant. Die Höhe der Armington-Elastizität verhält sich dabei umgekehrt proportional zur Höhe der Marktmacht eines Importeurs, wobei jedoch der große Importeur auch indirekt von der geänderten Wettbewerbsposition (d.h. niedrigerer Marktmacht) des großen Exporteurs profitieren kann (Fahlbusch 2007). 
Sebastian Hess - 978-3-631-75694-2

Downloaded from PubFactory at 01/11/2019 02:52:17AM

via free access 


\section{Weiterer Forschungsbedarf}

Die anonymen Gutachter des Journals „The World Economy“ haben Möglichkeiten zur Weiterentwicklung der Meta-Analyse vorgeschlagen:

\subsection{Mögliche Weiterentwicklungen im Bereich der Erhebung und Standardisierung der Meta-Daten:}

Die Abbildung dynamischer Szenarien kann verbessert werden, indem die innerhalb dynamischer Szenarien verwendeten Basis-Projektionen in der Referenzdatenbank präziser nachempfunden werden. Hierzu könnte an Stelle eines allgemeinen durchschnittlichen Wirtschaftswachstums von 2 Prozent jährlich auf länderspezifische Projektionen zurückgegriffen werden. Solche Projektionen werden insbesondere von der Weltbank erstellt und fließen in Simulationsexperimente zur Baseline-Projektion ein. Eine weitgehende unverzerrte Abbildung sollte daher in den Meta-Daten möglich sein. Die Zusammenfassung von modellierten Primärfaktoren innerhalb angewandter Gleichgewichtsmodelle, welche über die Aggregation von „Boden“, „Arbeit" und „Kapital“" hinausgehen, sollte verfeinert werden, um Unterschiede zwischen verschiedenen Graden der Arbeitsspezialisierung (skilled/unskilled) sowie weiteren, insbesondere in partiellen Modellen vorkommenden Beschränkungen (z.B. Quoten) besser als gegenwärtig durch eine einzelne Dummy-Variable abzubilden. Die Erfassung von Ansätzen innerhalb der Literatur zur Abbildung von NTB-Protektion kann eventuell durch Bildung von Kategorien oder das Einführen von Dummys für jede Studie innerhalb der Regression besser approximiert werden, als dies gegenwärtig über die Referenzdatenbank geschieht. Dies wird zumindest teilweise einen quantitativen Vergleich der gegenwärtig für Doha Szenarien existierenden Modellierungsansätze von NTB Protektion ermöglichen.

Die abhängige Variable sollte eine genauere Standardisierung erfahren, als dies in der Regression (Tabelle 6) erfolgt ist. Die Auswirkungen von Handelsliberalisierung auf ein Land sind eine Folge seiner Ausgangsprotektion, der Protektion seiner wichtigsten Handelspartner sowie seiner Nettohandelsposition. Die Abbildung dieser Unterschiede erfolgte in der Regression in Tabelle 6 ausschließlich durch die Proxy-Variable „Handelsvolumen eines Landes“. Alternativ kann diese Variable, wie durch einen Gutachter vorgeschlagenen, durch Fixed Effects abgebildet werden, sofern die Referenzdatenbank keine genauere Approximation dieser länderspezifischen Unterschiede zulässt. 


\subsection{Weiterentwicklung des ökonometrischen Meta-Modells:}

Im Rahmen der gewählten Abbildung des Meta-Datensatzes durch ein lineares Regressionsmodell muss berücksichtigt werden, dass aus jeder Publikation unterschiedlich viele Observationen in den Datensatz eingehen und diese auf verschiedene Weise miteinander korreliert sind. Diese Korrelation findet sich einerseits auf der Ebene jeder Publikation (durch gleiche Aggregation der Regionen und Sektoren sowie wichtiger Modellspezifikationen). Andererseits besteht diese Korrelation auf Ebene eines jeden Simulationsexperiments (durch experimentspezifische Closures und Politikänderungen). Diese verschiedenen Korrelationen sollten umfassender als nur durch das gegenwärtige Gewichtungsschema [1/Zahl der Observationen je Studie] abgebildet werden. Möglichkeiten hierzu bestehen durch Einsatz verschiedener Gewichtungsschemata sowie evtl. durch Transformation der abhängigen und der erklärenden Variablen, beispielsweise anhand einer Box-Cox-Transformation. Box-Cox-Transformationen dienen zur Identifikation angemessener Transformationsparameter $(\lambda)$, durch welche evtl. eine nicht vorliegende Normalverteilung der Fehlerterme erreicht, unterschiedliche Fehlertermvarianzen homogenisiert und evtl. Nicht-Linearitäten innerhalb der Daten linearisiert werden können (Kutner et al. 2005, S. 134).

Bisher wurden für die Regressionen in Tabelle 6 Box-Cox-Transformationen eingesetzt und ergaben für den „mit $B+L$ “ Datensatz ein $\lambda$ von 0,13 , was (nahe 0 ) auf eine logarithmische Transformation der abhängigen Variable hindeutet. Für den „ohne B $+\mathrm{L}$ “ Datensatz ergab sich ein $\lambda$ von 0,35 , was relativ nahe an 0,5 liegt und darauf hindeutet, dass in diesem Datensatz anhand der Quadratwurzel transformiert werden müsste (vgl. Kutner et al. 2005). Problematisch bei Box-Cox sowie logarithmischen Transformationen ist die Tatsache, dass viele Observationen in der abhängigen Variable negative Werte aufweisen und zunächst beispielsweise durch Addition des niedrigsten Wertes transformiert werden müssen. Die Interpretation von auf diese Weise geschätzten Koeffizienten wird hierdurch keineswegs erleichtert. Der Einsatz modifizierter BoxCox-Transformationen, welche auch negative Werte zulassen, stellt ein Ziel für die zukünftige Forschung dar.

\subsection{Forschungsbedarf im Bereich der Response-Surface- Modellierung:}

Die durchgeführten Response-Surface Applikationen wurden bisher nur anhand vergleichsweise einfacher Simulationsmodelle durchgeführt; dabei wurden Zoll- 
sätze und wichtige Parameter innerhalb plausibler Grenzen variiert. Jedoch wurde die Abhängigkeit simulierter Ergebnisse von weiteren in der Meta-Analyse als wichtig identifizierten Bestandteilen eines Simulationsexperiments nicht mit einbezogen. Dies betrifft insbesondere Closure Rules und Basisdaten.

Hinsichtlich unterschiedlicher Closure Rules sollte es ohne weiteres möglich sein, die vorgestellten Ansätze auf Gleichgewichtsmodelle mit komplexeren Closure-Spezifikationen zu übertragen; im Falle von Basisdaten, Zöllen, Handelsmengen, etc. wäre dies ebenfalls vielversprechend, wie jüngere Ansätze zur Evaluation verfügbarer Datengrundlagen (z.B. Brockmeier und Pelikan 2006) nahe legen. Technisch gestaltet sich die Konstruktion eines solchen BasisdatenResponse-Surface jedoch sehr aufwendig, da die ausbalancierte Struktur der Social-Account-Matrix (SAM), welche den meisten angewandten allgemeinen Gleichgewichtsmodellen zu Grunde liegt, beibehalten werden muss. Hierzu ist die Programmierung einer Routine notwendig, welche zufällige Werte für Basisdaten (Produktionswerte, Handelsmengen, etc.) so wählt, dass die Nebenbedingungen einer SAM eingehalten werden. Sollte dies gelingen, ohne dass der Zufallscharakter der auf diese Art erzeugten Basisdaten grundsätzlich verloren geht, könnte beispielsweise ein single country CGE Modell anhand dieser zufälligen Basisdaten in ein Response-Surface überführt werden, wobei die Interaktionseffekte zwischen Politikvariablen, (Armington-)Elastizitäten und Basisdaten ökonometrisch untersucht werden können. Predicted values dieses Modells könnten für jedes Land der Erde anhand weniger Koeffizienten abgelesen werden. Die Programmierarbeit $\mathrm{zu}$ einer solchen Response-Surface făhigen SAM wurde im Juni 2007 begonnen. Dazu wird das „GTAP in Excel Single Country CGE“ (van der Mensbrugghe; vgl. www.intereconomics.com/ handbook/Models/Index.htm) mit einer VBA Routine verwendet. Ergebnisse liegen allerdings bisher nicht vor.

Mit komplexeren Response-Surface Applikationen steigen die Anforderungen an eine sorgfältige Versuchsplanung, insbesondere weil die Rechnerkapazität schnell zu einem begrenzenden Faktor für die Zahl der innerhalb eines überschaubaren Zeitraumes durchführbaren Simulationen wird. Möglichkeiten für experimentelle Designs, die mit besonders wenigen Versuchsdurchführungen (Simulationen) auskommen, sind in Kutner et al. (2005) sowie Kleijnen et al. (2005) ausführlich beschrieben. 
Sebastian Hess - 978-3-631-75694-2

Downloaded from PubFactory at 01/11/2019 02:52:17AM

via free access 


\section{Fazit}

Das Forschungsprojekt „Meta-Analyse angewandter Gleichgewichtsmodelle des internationalen Agrarhandels" hat Antworten auf Fragen hinsichtlich der Rolle angewandter Gleichgewichtsmodelle für die quantitative Politikfolgenabschätzung gesucht. Diese Fragen wurden anhand eines repräsentativen Literatursamples der WTO Doha-Runde durch eine ökonometrische Meta-Analyse untersucht; auf hierbei gewonnene Erkenntnisse wurde aufgebaut, indem ResponseSurface Techniken mit Erkenntnissen der Meta-Analyse kombiniert wurden, woraus sich weitere und teilweise bisher ungelöste Forschungsfragen ergaben. Die ursprünglichen Forschungsfragen des Projektes können nach gegenwärtigem Erkenntnisstand folgendermaßen beantwortet werden:

- Transparenz: Kann das Zustandekommen von Simulationsergebnissen angewandter Gleichgewichtsmodelle zu Fragen der Wohlfahrtswirkungen multi-regionaler Handelsliberalisierung systematisch erklärt und über Studien und Modelle hinweg miteinander verglichen werden?

Die durchgeführte Meta-Analyse hat gezeigt, dass die in der Literatur verbreitete Antizipation nahezu willkürlich streuender Simulationsergebnisse das Resultat qualitativer Vergleiche ist, welche die Komplexität der meisten Simulationsexperimente unzulässig vereinfachen und noch dazu häufig anhand nicht repräsentativ ausgewählter Literaturlisten angestellt werden. Die multivariate Erfassung wichtiger Charakteristika eines Simulationsexperiments ist anhand solcher, gegenwärtig verbreiteter qualitativer Ergebnisvergleiche jedoch kaum sinnvoll möglich, wie die Meta-Analyse gezeigt hat.

Meta-Analyse hingegen kann solche Vergleiche ermöglichen, sofern insbesondere für den tatsächlichen Umfang der betrachteten Liberalisierungsexperimente kontrolliert wird. Wie die Ergebnisse für ein repräsentatives Literatursample gezeigt haben, kann Meta-Analyse auf die Frage „Welches sind die wichtigsten Charakteristika eines Simulationsexperiments, die das Zustandekommen bestimmter Simulationsergebnisse determinieren? " zumindest teilweise systematische Antworten geben.

In diesem Zusammenhang hat die durchgeführte Meta-Analyse den Einfluss innerhalb der Literatur häufig diskutierter Modellcharakteristika empirisch bestätigen und quantifizieren können; insbesondere die Rolle der ArmingtonElastizitäten, innerhalb der Modellierungsliteratur seit jeher kontrovers diskutiert, bestätigte sich empirisch als besonders bedeutend. In diesem Zusammenhang spielten Interaktionseffekte mit wichtigen Modellierungsansätzen, wie bei- 
spielsweise dem Unterschied zwischen der Annahme von konstanten oder steigenden Skalenerträgen, eine wichtige Rolle. Die Simulationsergebnisse innerhalb des Literatursamples unterliegen folglich dem systematischen Einfluss wichtiger Annahmen, welche innerhalb der Literatur verbreitet sind und - gemäß den Ergebnissen der Meta-Analyse - auch in sehr unterschiedlichen Modellkonzepten und Simulationsexperimenten eine ähnliche Wirkung auf die Ergebnisse entfalten.

- Empirie: Welche Aussagen können anhand bisher existierender Studien über durchschnittlich $\mathrm{zu}$ erwartende, simulierte Einkommens- (bzw. Wohlfahrts-)veränderungen in

a) der Welt als geschlossener Volkswirtschaft und

b) innerhalb einzelner Länder getroffen werden?

Predicted Values der Meta-Regression für Weltwohlfahrtsänderungen haben gezeigt, dass die im Mittel des Literatursamples ausgewiesenen numerischen Ergebnisse unterschiedlicher Modellansätze für eine vollständige Liberalisierung aller Zölle unter Verwendung der GTAP 6 Datenbank etwa im Bereich zwischen 130 Mrd. US-Dollar und 255 Mrd. US-Dollar (Preise 2001) schwanken, aber dass die Bandbreite dieser Ergebnisse auch anhand des komparativstatischen, CRTS (,Standard“-) GTAP Modells simuliert werden könnte, indem die Armington-Elastizitäten gegenüber den Standardwerten etwa vervierfacht würden.

Innerhalb des Literatursamples durchschnittlich zu erwartende Simulationsergebnisse für einzelne Länder und aggregierte geografische Regionen können errechnet werden, indem beispielsweise die Maximalwerte erklärender Variablen innerhalb des Samples für simulierte Politikänderungen angenommen und um die in Anhang 2 ausgewiesenen Fixed Effect Koeffizienten ergänzt werden; um präzisere Vergleiche bisher nicht aufgenommener Studien durchfuhren zu können, ist eine Approximation der Liberalisierungsszenarien anhand der zu diesem Zweck entwickelten Referenzdatenbank notwendig.

Die durchgeführten Response-Surface Studien haben ferner anhand einfach strukturierter Modelle gezeigt, dass simulierte Wohlfahrtseffekte selbst dann in großer Bandbreite schwanken, wenn nur wenige Parameter innerhalb plausibler Intervalle gleichzeitig variiert werden. Die Durchfuhrung ähnlicher Experimente mit komplexeren Simulationsmodellen steht jedoch noch aus. Als Gesamtergebnis des durchgeführten Projektes muss betont werden, dass Simulationsergebnisse angewandter Gleichgewichtsmodelle zwar empirische Ergebnisse zu simulierten Politikänderungen hervorbringen, aber diese Ergebnisse auf keinen Fall als ingenieurtechnische Messungen fehlinterpretiert werden sollten. 
Vielmehr scheint die besondere Stärke angewandter Gleichgewichtsmodelle in ihrer Möglichkeit zu liegen, komplexe Interaktionen wirtschaftspolitischer Zusammenhänge anhand realistischer Daten näherungsweise abbilden zu können. Die wenigsten Studien innerhalb des untersuchten Literatursamples stellen jedoch diese von Devarajan und Robinson (2002) und Francois (2004) besonders betonte Stärke in den Mittelpunkt von Politikanalysen zur Doha-Runde; vielmehr überwiegen im Grós der analysierten Studien ausführliche Diskussionen der ausgewiesenen numerischen Ergebnisse, beispielsweise im Vergleich zu inhaltlich ähnlichen Vorgängerstudien. Dies deutet darauf hin, dass möglicherweise überhöhte Erwartungen hinsichtlich der gegenwärtig erzielbaren Genauigkeit simulierter Politikfolgenabschätzungen weit verbreitet sind, und zwar sowohl unter Modellierern als auch unter Nicht-Modellierern.

Die durchgeführte Meta-Analyse bestätigt aufgrund der allgemein großen Abhängigkeit simulierter Ergebnisse von verwendeten Modellspezifikationen und Daten daher die Einschätzungen einiger Autoren (z.B. Devarajan und Robinson 2002, Sumner und Tangermann 2002, Harrison, Rutherford und Tarr 2003, Francois 2004), wonach eine sinnvolle Interpretation simulierter numerischer Ergebnisse nicht ohne ein Mindestverständnis des zu Grunde liegenden Simulationsexperiments vorgenommen werden kann und ein sinnvoller Vergleich simulierter Ergebnisse unterschiedlicher Studien nur möglich ist, nachdem fur die wichtigsten Unterschiede im jeweiligen Simulationsaufbau kontrolliert worden ist.

- Interpretation: Unter welchen Bedingungen werden simulierte Veränderungen in der Wirklichkeit eintreten?

Hinsichtlich der Zuverlässigkeit von Simulationsergebnissen zeigt der gegenwärtige Stand der Forschung deutlich, dass eine ex post Validierung anhand tatsächlich eingetretener Effekte zwar eine wünschenswerte Form der Modellvalidierung darstellt, die in diesem Zusammenhang auftretenden methodischen Schwierigkeiten jedoch beträchtlich sind. Simulationsergebnisse sind folglich grundsätzlich als hypothetische Szenarien zu betrachten.

Die Ergebnisse des Forschungsprojektes haben auch gezeigt, dass sich die Frage nach der allgemeinen Zuverlässigkeit von Simulationsergebnissen im Hinblick auf diejenigen Personen stellt, die aus einem Simulationsexperiment Erkenntnisse über die Auswirkungen von Politikänderungen gewinnen sollen: Diese Personen müssen in die Lage versetzt werden, den wichtigsten Kausalitäten im Zusammenhang mit der Entstehung von Simulationsergebnissen zu folgen, um eine Bewertung der Realität anhand dieser Ergebnisse vornehmen zu können. 
Aus diesem Grund führt die Öffnung der Black Box durch Meta-Analysen und Response-Surface Methoden allgemein zu einer Verbesserung des Verständnisses dieser Kausalität innerhalb angewandter Gleichgewichtsmodelle. Dies kann, verglichen mit gegenwärtig etablierten qualitativen Methoden, innerhalb des politischen Entscheidungsfindungsprozesses zu einer effizienteren Verwendungen der anhand von Simulationsexperimenten gewonnenen Erkenntnisse führen.

Die umfangreiche Literatur zur Rolle der Armington-Elastizitäten und der Rolle der Armington-Annahme innerhalb angewandter Gleichgewichtsmodelle macht aber auch deutlich, dass sich durch diese Modellspezifikation wahrscheinlich Interaktionen ergeben, welche auch bei profunder Kenntnis der theoretischen Zusammenhänge innerhalb eines Handelsmodells evtl. a priori nur schwer einschätzbar sind. Dies widerspricht der Auffassung, dass der Aufwand, mit welchem globale Simulationsmodelle entwickelt werden, nicht zu rechtfertigen sei, da sich "die Ergebnisse auch einfacher kalkulieren lassen“ (Taylor und von Arnim 2006, $\Delta$ Wohlfahrt $=1 / 2\left(\Delta \mathrm{P}^{*} \Delta \mathrm{Q}\right)$,"back-of-the-envelope“ Approximation anhand des folgenden Zusammenhangs: ; vgl. Nicholson 1998).

Die komplexen Interaktionseffekte, welche sich insbesondere durch die Armington-Annahme innerhalb angewandter Gleichgewichtsmodelle ergeben, verstärken die Bedeutung simulierter ToT-Effekte im Vergleich zur Bedeutung der Harberger-Effekte deutlich (verglichen mit einem Nicht-ArmingtonModell), was in der Literatur als qualitative Erkenntnis formuliert wird (Zhang 2006) und durch ein Response-Surface Experiment bestätigt wurde. Evtl. entstehende numerische Ähnlichkeiten zwischen „back-of-the-envelope“ Berechnungen und simulierten Effekten sind, wie viele numerische Effekte innerhalb des Meta-Datensatzes, ohne empirische Prüfung der zu ihrer Entstehung führenden Kausalität nicht von rein zufälliger Koinzidenz zu unterscheiden. Dies bedeutet jedoch nicht, dass angewandte Gleichgewichtsmodelle per se realitätsnähere Ergebnisse ausweisen, als dies anhand von „back-of-the-envelope“ Berechnungen möglich wäre.

Dieser Realitätsbezug simulierter Ergebnisse hängt vielmehr davon ab, in wie weit der Betrachter grundsätzlich die Armington-Annahme als modellhaftes Abbild der Wirklichkeit akzeptiert oder beispielsweise vollkommene Substituierbarkeit inländisch erzeugter durch ausländische Güter unterstellt. Die Errechnung von Wohlfahrtsänderungen anhand von "back-of-the-envelope" Approximationen, anhand eines Armington-Modells oder anhand eines Modells mit perfekter Gütersubstitution stellt drei die Entstehung der Ergebnisse sehr entscheidend beeinflussende Annahmen dar. Die Tatsache, dass numerische Ergebnisse dieser Modelle dennoch eventuell ähnliche Größenordnungen aufweisen, sagt nichts bezüglich der Aussagekraft jedes einzelnen dieser drei Modelle über die Wirklichkeit. Simulierte Ergebnisse, gleichgültig auf Basis wel- 
ches Modells, sind daher so realistisch wie die Annahmen, welche zu ihrer Errechnung getroffen wurden.

- Methodische Weiterentwicklung: Durch welche Voraussetzungen kann die allgemeine Akzeptanz angewandter Gleichgewichtsmodelle als wissenschaftliche Methode zur quantitativen Politikfolgenabschätzung verbessert werden?

Vor dem Hintergrund vieler lückenhaft dokumentierter Simulationsexperimente innerhalb des Literatursamples stellt sich die Frage, ob im Bereich der modellgestützten Politikberatung insgesamt die Dokumentationspflicht für empirische Ergebnisse weiterhin relativ unverbindlich gehandhabt werden sollte. Zumindest erscheint es fragwürdig, ob der empirische Aspekt modellgestützter Politikfolgenabschätzung seine Glaubwürdigkeit dauerhaft aufrecht erhalten kann, ohne verbindliche Dokumentationsstandards einzuführen und ohne eine Reproduzierbarkeit der Simulationsexperimente allgemein zu ermöglichen. Das GTAP-Netzwerk hat jüngst bereits eine verbesserte Möglichkeit zur Hinterlegung von Simulations-experimenten auf seiner Internetplattform mit dem Hinweis eingerichtet, dass verbesserte Dokumentation die allgemeine Glaubwürdigkeit von Simulations-experimenten erhöhen wird: ,...Increasingly, however, Journal editors are insisting that authors make their applications publicly available. Here at the Center we also feel that applications should be made publicly available as it helps to increase the credibility of CGE work in general..." (vgl. GTAP-L Emailliste, Email vom 22. 3. 2007).

Das Forschungsprojekt hat ferner gezeigt, dass die vollständige Erfassung aller Details eines Simulationsexperiments einem trade-off hinsichtlich der benötigten Kapazitäten zur Informationsverarbeitung unterliegt. Dies bedeutet, dass lückenlose Dokumentation von Simulationsergebnissen und experimentellem Aufbau insbesondere die Nachfrageseite nach Simulationen noch immer vor das Problem hoher Spezialisierungskosten stellt, welche zunächst getragen werden müssen, bevor die dokumentierten Informationen für eine Ergebnisevaluation genutzt werden können.

Die durchgefuhrte Meta-Analyse hat somit nicht nur ein wesentliches Stück der Black-Box von Doha-Simulationen geöffnet, sondern kann auch als vergleichsweise einfach zu handhabendes Werkzeug gebraucht werden, um die Ergebnisse künftiger Studien mit einem repräsentativen Sample der Modellierungsliteratur unter der Doha-Runde approximativ zu vergleichen. Die durchgefuhrte Meta-Analyse kann bei geringem zusätzlichem Aufwand fortgefürt und um neue Modellcharakteristika (z.B. die GTAP 7 Datenbank) erweitert werden, da das System der Referenzdatenbanken sowie der Fragebogen zur einheitlichen Erfassung von Simulationsexperimenten über Studien hinweg inzwischen etab- 
liert ist. Andererseits dürfte sich der marginale Erkenntnisgewinn aus einer Erweiterung des Literatursamples kaum noch verbessern, sofern nicht die durchschnittlich aus einer Studie gewonnenen und in der Meta-Analyse verarbeiteten Informationen ausgeweitet werden können.

Der Einsatz von Meta-Analysen und Response-Surface Erstellung ermöglicht keine eigenen Politikfolgenabschätzungen. Eine sorgfältige Anwendung dieser Methoden, unterstützt durch verbesserte Dokumentationsstandards, kann jedoch multivariate Vergleiche einer heterogenen und bis dato für ihre Intransparenz berüchtigten Literatur ermöglichen. Durch die Identifikation von „Ausreißern“ kann ferner systematisch hinterfragt werden, welche Rolle selten verwendete Modellansätze für die Größenordnung simulierter Ergebnisse spielen. Da Simulationsergebnisse per Definition hypothetischer Natur sind, kann auch die Meta-Analyse keine Aussage darüber treffen, welche Effekte wahr-scheinlicher als andere eintreten werden, aber Meta-Analyse und MetaResponse-Modellierung können zeigen, durch welche Annahmen bestimmte numerische Ergebnisse besonders stark determiniert werden, sodass Diskussionen im Rahmen des politischen Entscheidungsfindungsprozesses auf diese Annahmen konzentriert werden können.

Sollten sich diese meta-analytischen Methoden im Zusammenhang mit der Verwendung angewandter Gleichgewichtsmodelle als eine solche Evaluationsmethode etablieren, würden möglicherweise überhöhte Erwartungen an die Aussagekraft simulierter Ergebnisse gedämpft und fundamentale Skepsis beseitigt. Die Verwendung angewandter Gleichgewichtsmodelle ökonomische Methode zur quantitativen Politikfolgenabschätzung würde somit effizienter - mit allen zu erwartenden positiven Auswirkungen auf die politischen Prozesse selbst.

Es wird sich jedoch erst herausstellen müssen, ob dieser Ansatz insbesondere das Umfeld modelltechnisch wenig erfahrener Entscheidungsträger der Handelsadministrationen erreichen kann, und ferner, ob diese Methoden zur Transparenzschaffung weiterentwickelt werden und Verbreitung finden oder ob angewandte Gleichgewichtsmodelle selbst ihre gegenwärtige Rolle in der Politikberatung teilweise verlieren werden; evtl. zu Gunsten einer Renaissance qualitativer, theoretischer Analysen?

Meta-Analyse, trotz aller praktischen Probleme bei ihrer Umsetzung, macht insbesondere die starke Abhängigkeit simulierter Ergebnisse von einigen ganz bestimmten Modellcharakteristika deutlich und unterstreicht somit, dass selbst die komplexesten Simulationsmodelle dem der Gesellschaft verantwortlichen Ökonomen (Public Economist, vgl. Giersch 1990) sowie dem an rationalen Entscheidungen interessierten Politiker den Einsatz des eigenen Verstandes zur Analyse von Politik und zum Treffen von Entscheidungen nicht abnehmen können. 


\section{Literaturverzeichnis}

$A B A R E$ (2000): The Impact of Agricultural Trade Liberalisation on Developing Countries. Australian Bureau of Agricultural and Resource Economics, Canberra. Autoren: Freeman, F., Melanie, J., Roberts, I., Vanzetti, D., Tielu, A.und Beutre, B.

Ackerman, F. (2005): The Shrinking Gains from Trade: A Critical Assessment of Doha Round Projections. Working Paper No. 05-01. Global Development and Environment Institute, Tufts University.

Akerlof, G. (1970): The Market for 'Lemons': Quality Uncertainty and the Market Mechanism. Quarterly Journal of Economics 84: 488-500.

Alston, J. M., Chang-Kang, C., Marra, M. C., Pardey P. G. und Wyatt, T. (2000): A Meta-Analysis of Rates of Return to Agricultural R\&D - Ex Pede Herculem? International Food and Policy Research Institute, Washington D.C.

Alston, J. M., und Hurd, B. (1990): Some Neglected Social Costs of Government Spending in Farm Programs. American Journal of Agricultural Economics 72(1): 149-160.

Anderson, J. E. (2006): Consistent Policy Aggregation. Working Paper, Boston College and NBER, Cambridge.

Anderson, J. E., und Neary, J. P. (2003): The Mercantilist Index of Trade Policy. International Economic Review 44(2): 627-649.

Anderson, J. E. und van Wincoop, E. (2001): Borders, Trade and Welfare. NBER Working Paper Series 8515, Cambridge.

Anderson, K., Francois, J., Hertel, T., Hoekman, B. und Martin W. (2000): Potential Gains from Trade Reform in the New Millennium. Paper presented at the Third Annual Conference on Global Economic Analysis, Monash University, Australien, 27. - 30. Juni.

Anderson, K., Hoekman, B. und Strutt, A. (1999): Agriculture and the WTO: The Next Steps. Paper prepared for the Second Annual Conference on Global Economic Analysis, Avernaes Conference Centre, Helnaes, Dänemark, 20. - 22. Juni.

Ardelean, A. (2007): How Strong is the Love of Variety? Presented at the 10th Annual Conference on Global Economic Analysis, Purdue University, USA. https://www.gtap.agecon.purdue.edu/resources/res_display.asp? RecordID $=2447$. 
Armington, P. S. (1969): A Theory of Demand for Products Distinguished by Place of Production. IMF Staff Papers 16: 159-177.

Arndt, C. (1996): An Introduction to Systematic Sensitivity Analysis via Gaussian Quadrature. GTAP Technical Paper No. 2. https://www.gtap. agecon.purdue.edu/resources/res_display.asp?RecordID $=305$.

Backhaus, K., Erichson, B., Plinke, W. und Weiber, R. (2005): Multivariate Analysemethoden. Eine anwendungsorientierte Einführung. Springer, 11. Auflage, Berlin.

Banks, J. (1998): Handbook of Simulation - Principles, Methodology, Advances, Applications, and Practice. John Wiley \& Sons, New York.

Banse, M. und Tangermann, S. (1996): Agricultural Implication of Hungary's Accession to the EU - Partial versus General Equilibrium Effects. Working Paper No.1/1, Institut für Agrarökonomie, Universität Göttingen.

Bateman, I. und Jones, A. (2003): Contrasting Conventional with Multi-Level Modelling Approaches to Meta-Analysis: Expectation Consistency in UK Woodland Recreation Values. Land economics: a quarterly journal of planning, housing \& public utilities 79: 235-258.

Bchir, M. H., Jean, S. und Laborde, D. (2006): Binding Overhang and TariffCutting Formulas. Review of World Economics 142(2): 207-232.

Bernhardt, I. und Jung, B. S. (1979): The Interpretation of Least Squares Regression With Interaction or Polynomial Terms. The Review of Economics and Statistics 61(3): 481-483.

Bertelsmeier, M., Kleinhanss, W. und Offermann, F. (2003): Aufbau und Anwendung des FAL-Modellverbunds für die Politikberatung. Agrarwirtschaft 4(8): 175-184.

Bouët, A. (2006): What can the Poor Expect from Trade Liberalization? Opening the 'Black Box' of Trade Modelling. MTID Discussion Paper No. 93, IFPRI (International Food Policy Research Institute), Washington D.C. Bouët, A. und Krasniqi, V. B. (2006): Is Trade Pessimism Justified? Opening the "Black Box" of Trade Modeling. Paper prepared for the Joint International Institute for Sustainable Development (IISD) and the World Trade Organization (WTO) Seminar on Modeling the Gains from Trade Liberalization, Genf.

Box, G. und Wilson, $K$. (1951): On the Experimental Attainment of Optimum Conditions (with discussion). Journal of the Royal Statistical Society Series B 13(1): 1-45.

Breiman, L., Friedman, J. H., Olshen, R. A. und Stone, C. J. (1984): Classification and Regression Trees. Wadsworth, Belmont, USA.

Brockmeier, M. (2003): Ökonomische Auswirkungen der EU-Osterweiterung auf den Agrar- und Ernährungssektor- Simulationen auf Basis eines 
Allgemeinen Gleichgewichtsmodells. Habilitationsschrift, Institut für Agrarpolitik und Marktforschung. Fachberich Agrarwissenschaften, Ökotrophologie und Umweltmanagement, Justus-Liebig-Universität Gießen.

Brockmeier, M. und Pelikan, J. (2006): Agricultural Market Access: A Moving Target in the WTO Negotiations? Contributed paper presented at the 26th IAAE (International Association of Agricultural Economics) Conference, Gold Coast, Australien, 12. - 18. August.

Brown, D. K. (1987): Tariffs, the terms of trade, and national product differentiations. Journal of Policy Modeling 9(3): 503-526.

Brown, D. K., Deardorff, A. V. und Stern, R. M. (2003): Developing Countries' Stake in the Doha Round. Discussion Paper No. 495, Gerald R. Ford School of Public Policy, Research Seminar in International Economics, University of Michigan.

Brown, D. K., Deardorff, A. V. und Stern, R. M. (2002): Computational Analysis of Multilateral Trade Liberalization in the Uruguay Round and Doha Development Round. Discussion Paper No. 489, Gerald R. Ford School of Public Policy, Research Seminar in International Economics, University of Michigan.

Brown, D. K., Deardorff, A. V. und Stern, R. M. (2001): CGE Modeling and Analysis of Multilateral and Regional Negotiating Options. Discussion Paper No. 468, Gerald R. Ford School of Public Policy, Research Seminar in International Economics, University of Michigan.

Brown, D. K., Kiyota, K. und Stern, R. M. (2005): Computational Analysis of the U.S. Bilateral Free Trade Agreement with Central America, Australia, and Marocco. Discussion Paper No. 527, Ford School of Public Policy, Research Seminar in International Economics, University of Michigan.

Bureau, $J$. (2006): On models for trade policy debate (and on possible networks to improve them). http://www.enarpri.org/Publications/PPTNo18.ppt

Button, K. J. und Jongma, S. M. (1995): Meta-Analysis Methodologies and Microeconomics. Discussion Paper, Tinbergen Institute, Amsterdam.

Chalmers, I. und Altman, D. G. (Hrsg.) (1995): Systematic Reviews. BMJ Publishing Group, London.

Charlton, A. H. und Stiglitz, J. E. (2005): A Development-friendly Priorisation of Doha Round Proposals. The World Economy 28(3): 293-312.

Cipollina, M. Und Salvatici, L. (2006): Reciprocal Trade Agreements in Gravity Models: A Meta-Analysis". TradeAG Working Paper No. 06/12 Available at SSRN: http://ssrn.com/abstract $=950929$

Copenhagen consensus (2007): Subsidy and Trade Barrier Removal. Results of the Copenhagen Consensus 2004. www.copenhagenconsensus.com/ Default.aspx?ID=227. 
Cooper, H. (1998): Synthesizing Research - A Guide for Literature Reviews. SAGE Publications, 3. Auflage, Thousand Oaks, USA.

Corden, W. M. (1974): Trade Policy and Economic Welfare. Oxford University Press.

Decaluwé, B., Martens, A. und Monette, M. (1988): Macroclosures in Open Economy CGE Models: A Numerical Reappraisal. International Journal of Development Planning Literature 3(2): 69-90.

Dempster, A., Laird, N. und Rubin, D. (1977): Maximum-Likelihood from Incomplete Data via the EM Algorithm. Journal of the Royal Statistical Society Series B 39(1): 1-38.

Devarajan, S. und Robinson, S. (2002): The Influence of Computable General Equilibrium Models on Policy. TMD Discussion Paper No. 98, IFPRI (International Food Policy Research Institute), Washington D.C.

Dewatripont, M. und Michel, G. (1987): On Closure Rules, Homogeneity and Dynamics in Applied General Equilibrium Models. Journal of Development Economics 26: 65-76.

Diao, X., Somwaru, A. und Roe, T. (2001): A Global Analysis of Agricultural Reform in WTO Member Countries. In: Burfisher, M. (Hrsg.): Agricultural Policy Reform in the WTO - The Road Ahead. Agricultural Economic Report No. 802, USDA-ERS (United States Department of Agriculture Economic Research Service), Washington D.C.

Dorman, $P$. (2001): The Free Trade Magic Act- In dubious study, first you see the benefits, then you don't. Economic Policy Institute, Washington, D.C.

Enneking, U. (1997): Effektive Suche nach agrarökonomischer Literatur - Ein Überblick mit technischen Hinweisen. Diskussionsbeitrag 9701, Institut für Agrarökonomie, Universität Göttingen.

Eysenck, H. J. (1995): Problems with Meta-Analysis. In: Chalmers, I. und Altman, D. G.: Systematic Reviews. BMJ Publishing Group.

Fahlbusch, M. (2007): Entwicklung eines Handelsmodell-Response-Surface in Excel VBA am Beispiel des GSIM Modells. Bachelorarbeit im wissenschaftlichen Studiengang Agrarwissenschaften an der Georg-AugustUniversität Göttingen.

Feenstra, R. C. (2006): New Evidence on the Gains from Trade. Review of World Economics 142 (4): 617-641.

Feenstra, $R$. C. (2003): Advanced International Trade: Theory and Evidence. Princton University Press.

Florax, R. J. C. M. (2002): Accounting for Dependence among Study Results in Meta-Analysis: Methodology and Applications to the Valuation and 
Use of Natural Resources. Research Memorandum 2002-5, Vrije Universiteit, Amsterdam.

Fox, J. (2002): Linear Mixed Models. http://cran.r-project.org/doc/contrib/FoxCompanion/appendix-mixed-models.pdf.

Francois, J. (2004): General Equilibrium Studies of Multilateral Trade Negotiations: Do They Really Help? In: Nelson, D. (Hrsg.): The Political Economy of Policy Reform. Elsevier, Amsterdam: 75-102.

Francois, J. (2001): The Economic Impact of New Multilateral Trade Negotiations. Final Report, Prepared for DG-II of the European Commission, Tinbergen Institute and Erasmus University, Rotterdam.

Francois, J. und Hall, K. (2003): Global Simulation analysis of Industry-Level Trade Policy, Tinbergen institute, CEPR, US commerce Department.

Francois, J. und Hall, K. (1997): Partial Equilibrium Modelling. In: Francois, J. und Reinert, K. A. (Hrsg.): Applied Methods for Trade Policy Analysis - A Handbook. Cambridge University Press, Cambridge: Kapitel 5.

Francois, J. und Reinert, K. A. (Hrsg.) (1997): Applied Methods for Trade Policy Analysis - A Handbook. Cambridge University Press, Cambridge.

Francois, J., van Meijl, $H$. und van Tongeren, F. (2005): Trade Liberalisation in the Doha Development Round, Technical Annex: An Overview of the Computational Model. Economic Policy 20(42): 349-391.

Frohberg, K. und Winter, E. (2004): Impacts of Croatia's Bi- and Multilateral Trade Agreements: Experiments with Different Trade Model Specifications. In: Dabbert, S., Grosskopf, W., Heidhues, F. und Zeddies, J. (Hrsg.): Perspektiven in der Landnutzung: Regionen, Landschaften, Betriebe; Entscheidungsträger und Instrumente. Landwirtschaftsverlag, Münster-Hiltrup: 637-647.

Gehlhar, M. (1997): Historical Analysis of Growth and Trade Patterns in the Pacific Rim: An Evaluation of the GTAP Framework. In: Hertel, T. W. (Hrsg.): Global Trade Analysis. Cambridge University Press, Cambridge: $349-363$.

Gersfelt, B., Lind, K., Frandsen, S., Jensen, H. und Yu, W. (2003): Policy Note on the Harbinson Draft. Briefing Paper for the COMESA Ministerial on WTO and EPAs, Nairobi.

Giersch, $H$. (1990): On Being a Public Economist. Lecture held at the prizeawarding ceremony of the Paolo Baffi International Prize for Economics 1989 with a Laudation by Edmond Malinvaud. Institut für Weltwirtschaft, Kiel.

Ginsburgh, V. und Keyzer, M. (1997): The Structure of Applied General Equilibrium Models. The MIT Press, Cambridge. 
Gohin, A. und Moschini, G. (2005): Evaluating the Market and Welfare Impacts of Agricultural Policies in Developed Countries: Comparison of Partial and General Equilibrium Measures. Review of Agricultural Economics 28(2): 195-211.

Goldberger, A. S. (1998): Introductory Econometrics. Harvard University Press, Cambridge.

Goldstein, H. (1995): Multilevel Statistical Models. Arnold, London.

Greene, W. (2003): Econometric Analysis. Prentice-Hall, 5. Auflage, Upper Saddle River, USA.

Gujarati, D. N. (2003): Basic Econometrics. McGraw-Hill, 4. Auflage, Boston, USA.

Harrison, G. W., Rutherford, T. F. und Tarr, D. G. (2003): Rules of Thumb for Evaluating Preferential Trading Arrangements: Evidence from Computable General Equilibrium Assessments. Cuadernos de Economia 40(121): 460-468.

Harrison, G. W., Rutherford, T. F. und Tarr, D. G. (1997): Quantifying the Uruguay Round. The Economic Journal 107(444): 1405-1430.

Heckelei, T., Witzke, H. und Henrichsmeyer, W. (Hrsg.) (2000): Agricultural Sector Modelling and Policy Information Systems. 65th European Seminar of the European Association of Agricultural Economists (EAAE), Bonn, 29. - 31. März.

Heckman, J. J. (2001): Micro Data, Heterogeneity, and the Evaluation of Public Policy: Nobel Lecture. Journal of Political Economy 109: 673-748.

Hedges, L. V. und Olkin, I. (1985): Statistical Methods for Meta-Analysis. AP Academic Press, London.

Hertel, T. W. (1999): Future Directions in Global Trade Analysis. Staff Paper \#99-8, Department of Agricultural Economics, Purdue University, West Lafayette, USA.

Hertel, T. W. (Hrsg.) (1997): Global Trade Analysis- Modelling and Applications. Cambridge University Press.

Hertel, T. W. (1989): Negotiating Reductions in Agricultural Support: Implications of Technology and Factor Mobility. American Journal of Agricultural Economics 71(3): 559-573.

Hertel, T. W., Hummels, D., Ivanic, M und Keeney, R. (2003): How Confident Can We Be in CGE-Based Assessments of Free Trade Agreements? GTAP Working Paper No. 26, Center for Global Trade Analysis, Purdue University, West Lafayette, USA.

Hertel, T. W., Keeney, R., Ivanic, M. und Winters, L. A. (2006): Distributional Effects of WTO Agricultural Reforms in Rich and Poor Countries. Policy Research Working Paper No. 4060, The World Bank, Washington D.C. 
Hess, S. (2006): Eine ökonometrische Analyse CGE- basierter Handelssimulationen im Rahmen der Doha- Runde. In: Bahrs, E., von Cramon-Taubadel, C., Spiller, A., Theuvsen, L. und Zeller, M. (Hrsg.): Unternehmen im Agrarbereich vor neuen Herausforderungen. Landwirtschaftsverlag, Münster-Hiltrup: 271-282.

Hess, S. (2005a): In the Jungle: Towards a Common Documentation Standard for CGE- based Experiments. 8th Annual Conference of Global Economic Analysis (GTAP), Lübeck, 9. - 11. Juni.

Hess, S. (2005b): An Econometric Model of CGE Simulations. XIth Conference of the European Association of Agricultural Economists (EAAE), Kopenhagen.

Hess, S. und von Cramon-Taubadel, S. (2007a): Meta-Analysis of General and Partial Equilibrium Simulations of Doha Round Outcomes. In: Otsuka, K. and Kalirajan, K. (Hrsg.): Contributions of Agricultural Economics to Critical Policy Issues (forthcoming). Blackwell, Malden, USA.

Hess, S. und von Cramon-Taubadel, S. (2007b): Doha Round Outcomes: A Meta-Analysis of General and Partial Equilibrium Simulations. Submitted to The World Economy.

Hess, S. und von Cramon-Taubadel, S. (2006): Meta-Analysis of General and Partial Equilibrium Simulations of Doha Round Outcomes. Invited Paper at IAAE Conference Gold Coast, Australien.

Heston, A., Summers, R. und Aten, B. (2006): Penn World Table Version 6.2. Center for International Comparisons of Production, Income and Prices, University of Pennsylvania, Philadelphia, USA.

Hsiao, C. (2003): Analysis of Panel Data. Cambridge University Press.

Kehoe, T. J. (2002): An Evaluation of the Performance of Applied General Equilibrium Models of the Impact of NAFTA. Paper Presented at the 5th Conference on Global Economic Analysis, Taipeh, 5. - 7. Juni. http://www.econ.umn.edu/ tkehoe.

Keynes, J. M. (1922): Introduction to Cambridge Economic Handbooks. Cambridge University Press.

Kleijnen, J. P. C., Sanchez, S. M., Lucas, T. W. und Cioppa, T. M. (2005): A User's Guide to the Brave New World of Designing Simulation Experiments. INFORMS Journal on Computing 17(3): 263-289.

Kremers, H., Nijkamp, P. und Rietveld, P. (2000): A Meta-Analysis of Price Elasticities of Transport Demand in a General Equilibrium Framework. Discussion Papers 00-060/3, Tinbergen Institute, Amsterdam.

Lamy, P. (2007): WTO News-Speeches, DG Pascal Lamy, G-8 summit Heiligendamm, Deutschland, 8. Juni. http://www.wto.org/english/news e/sppl_e/sppl63_e.htm. 
Little, R. (1992): Regression With Missing X's: A Review. Journal of the American Statistical Association 87(420): 1227-1237.

Lodefalk, M. und Kinnman, S. (2006): Economic Implications of the Doha Round. Kommerskollegium - Swedish National Board of Trade, Governmental Report, Stockholm.

Lucas, R. E. (1976): Econometric policy evaluation: a critique. Journal of Monetary Economics 12: 19-46.

Maindonald, J. und Braun, J. (2003): Data analysis and Graphics Using R - an

Example-based Approach. Cambridge Series in statistical and

Probabilistic Mathematics. Cambridge University Press.

Martin, W., van der Mensbrugghe, D. und Manole, V. (2003): Is the Devil in the

Details? Assessing the Welfare Implications of Agricultural Trade

Reforms. Contributed paper presented at the conference 'Agricultural policy reform and the WTO: where are we heading?', Capri, Italien, 23. - 26. Juni.

Martin, W. (1997): Measuring welfare changes with distortions. In: Francois, J. und Reinert, K. A. (Hrsg.): Applied Methods for Trade Policy Analysis - A Handbook. Cambridge University Press, Cambridge: Kapitel 5.

Mas-Colell, A., Whinston, M. D. und Green, J. R. (1995): Microeconomic Theory. Oxford University Press, New York.

McDonald, S. und van Tongeren, F. W. (2003): Alternative Closures in GTAP. Course material and lecture notes presented at the Twelfth Annual Short Course of Global Trade Analysis, Purdue University, Philadelphia, USA, 7. - 13. August.

McKitrick, R. R. (1998): The Econometric Critique of Computable General Equilibrium Modeling: The Role of Parameter Estimation. Economic Modeling 15: 543-573.

Moore, M. (2002): UN Financing for Development Conference summit-level opening session. A grand bargain: a new international deal. WTO News: Speeches-DG Mike Moore, Monterrey, 21. März 2002. http:// www.wto.org/english/news_e/spmm_e/spmm81_e.htm.

Nagarajan, N. (1999): The Millennium Round: An Economic Appraisal. Economic Paper Number 139, Europäische Kommission, Brüssel.

Nicholson, W. N. (1998): Microeconomic Theory- Basic Concepts and Extensions. The Drydon Press.

Nölle, F. und Banse, M. (2002): Wie gut lassen sich Agrarpolitische Reformen durch partielle Modelle abbilden? Eine ex-post Analyse der MacSharry Reform am Beispiel von ESIM. In: Brockmeier, M., Isermeyer, F. und von Cramon-Taubadel, S. (Hrsg.): Liberalisierung 
des Weltagrarhandels - Strategien und Konsequenzen. Landwirtschaftsverlag, Münster-Hiltrup: 509-511.

Ohlin, B. (1933): Interregional and International Trade. Harvard Econ. Studies 39. Harvard University Press.

Panagariya, A. und Duttagupta, R. (2001): The 'Gains' from Preferential Trade Liberalization in the CGE Models, where Do They Come from? In: Lahiri, S. (Hrsg.): Regionalism and Globalization Theory and Practice. Routledge, London: 39-60.

Pelikan, J. und Brockmeier, M. (2007): Methods to Aggregate Import Tariffs and their Impacts on Modeling Results. Presented at the 10th Annual Conference on Global Economic Analysis, Purdue University, West Lafayette, USA.

Piermartini, R. und Teh, R. (2005): Demystifying Modelling Methods for Trade Policy. Discussion Paper No. 10, WTO (World Trade Organization), Genf.

Pinheiro, J. C. und Bates, D. M. (2000): Mixed-Effects Models in S and S-Plus. Springer, New York.

Rattsø, J. (1982): Different Macroclosures of the Original Johansen Model and Their Impact on Policy Evaluation. Journal of Policy Modeling 4(1): 85-97.

Rawlings, O., Pantula, S. und Dickey, D. (1998): Applied Regression Analysis:

A Research Tool. Springer, 2. Auflage, New York.

Robinson, S. (1989): Multisectoral Models. In: Chenery, H. und Srinivasan, T.

N. (Hrsg.): Handbook of Development Economics. Elsevier, Band II, Amsterdam: 885-947.

Robinson, S. und Lofgren, H. (2005): Macro Models and Poverty Analysis: Theoretical Tensions and Empirical Practice. Development Policy Review 23(3): 267-283.

Ricardo, D. (1821): On the Principles of Political Economy and Taxation. John Murray, 3. Auflage, London. www.econlib.org/library/Ricardo/ricP. html

Sarker, R. und Surry, Y. (2006): Product Differentiation and Trade in Agri-Food Products: Taking Stock and Looking Forward. Journal of International Agricultural Trade and Development, 34(2): 39-78.

Schulz, M. (2003): Statistical Physics and Economics: Concepts, Tools, and Applications. Springer, New York.

Scollay, $R$ und Gilbert, J. (2001): An Integrated Approach to Agricultural Trade and Development Issues: Exploring the Welfare and Distribution Issues. Policy Issues in International Trade and Commodities Study Series No. 11, UNCTAD (United Nations Commission on Trade and Development), Genf. 
Scollay, R. und Gilbert, J. (2000): Measuring the Gains from APEC Trade Liberalization: An Overview of CGE Assessments. The World Economy 23(2): 175-197.

Shoven, J. B. und Whalley, J. (1984): Applied General-Equilibrium Models of Taxation and International Trade: An Introduction and Survey. Journal of Economic Literature 22: 1007-1051.

Stanley, T. D. (2005): Beyond Publication Bias. Journal of Economic Surveys 19(3): 309-345.

Stanley, T. D. (2001): Wheat from Chaff: Meta-Analysis As Quantitative Literature Review. Journal of Economic Perspectives 15(3): 131-150.

Stanley, T. D. und Jarrell, S. B. (1989): Meta-Regression Analysis: A Quantitative Method of Literature Surveys. Journal of Economic Surveys 3: 161-170.

Sumner, D. A. und Tangermann, S. (2002): International Trade Policy and Negotiations. In: Gardner, B. und Rausser, G. (Hrsg.): Handbook of Agricultural Economics. Elsevier, Amsterdam: 1999-2056.

Taylor, L. und von Arnim, R. (2006): Modelling the Impact of Trade Liberalisation - A Critique of Computable General Equilibrium Models. Oxfam International Research Report.

Thiam, A., Bravo-Ureta, B. und Rivas, T. (2001): Technical Efficiency in a

Developing Country Agriculture: A Meta-Analysis. Agricultural Economics 25: 235-243.

UNCTAD (2003): Back to Basics: Market Issues in the Doha Agenda. UNCTAD (United Nations Conference on Trade and Development), Genf. Autoren: Laird, S., Cernat, L. und Turrini, A.

van den Bergh, J., Button, K., Nijkamp, P. und Pepping, G. (1997): MetaAnalysis in Environmental Economics. Kluwer Academic Publishers, Dordrecht, Niederlande.

van Meijl, $H$. und van Tongeren, $F$. (2001): Multilateral Trade Liberalisation and Developing Countries: A North-South Perspective on Agriculture and Processing Sectors. Paper prepared for the Fourth Annual Conference on Global Economic Analysis, Purdue University, West Lafayette, USA, 27. - 29. Juni.

van Tongeren, F., van Meijl, H. und Surry, Y. (2001): Global Models Applied to Agricultural and Trade Policies: A Review and Assessment. Agricultural Economics 26: 149-172.

von Hayek, F. (1963): Wirtschaft, Wissenschaft und Politik. Freiburger Universitätsreden 34.

Wachter, $K$. W. (1988): Disturbed by Meta-Analysis. Science 241: 1407-1408. Wooldridge, J. M. (2002): Econometric Analysis of Cross Section and Panel Data. The MIT Press, Cambridge. 
WTO (2005): Doha Ministerial Declaration. http://www.wto.org.

$X u, Y$. (2002): A Comparison of Two Models of Intra-Industry Trade. Journal of International Trade \& Economic Development 11(4): 405-427.

Zellner, A. (1962): An Efficient Method of Estimating Seemingly Unrelated Regression Equations and Tests for Aggregation Bias. Journal of the American Statistical Association 57: 348-368.

Zhang, X. (2006): Armington Elasticities and Terms of Trade Effects in Global CGE Models, Productivity Commission Staff Working Paper, Melbourne, Australien. 
Sebastian Hess - 978-3-631-75694-2

Downloaded from PubFactory at 01/11/2019 02:52:17AM

via free access 


\section{Anhang A: Das Literatursample}

Es ist möglich, das nachfolgend aufgefuhrte Literatursample approximativ zu replizieren, indem auf alle Datenbanken in Tabelle 12 alle Suchworte aus Tabelle 13 angewendet werden und alle Treffer bis einschließlich Publikationsmonat Oktober 2006 abgespeichert werden. Die Treffer werden dann um Duplikate bereinigt und anhand der in Kapitel 3.3.2 beschriebenen Filterkriterien gefiltert.

Tabelle 12: Verwendete Literaturdatenbanken.

\begin{tabular}{|l|l|l|}
\hline AgEconSearch & OCLC Journal Articles ECO & KVK \\
\hline Econis & OCLC Papers & IBZ \\
\hline EconLit & OCLC Proceedings & JADE \\
\hline RePec Articles & OCLC World Cat & OLC \\
\hline RePec Working Papers & COPAC & SSCI \\
\hline JSTOR & WTO House Library & Google/ Google Scholar \\
\hline
\end{tabular}

Quelle: Eigene Zusammenstellung.

Tabelle 13: Matrix der verwendeten Suchworte.

Jedes der 16 Modelling-Keywords wird mit jedem der 10 Doha-Keywords kombiniert, wodurch 160 Suchwortkombinationen entstehen (beispielswiese applied model + doha agric* market ${ }^{*}$ ). Diese Kombinationen wurden durch automatisierte und teil-automatisierte Suchanfragen an alle ausgewählten Literaturdatenbanken gesendet; Erlăuterung siehe Kapitel 3.3.

\begin{tabular}{|l|l|l|l|}
\hline \multicolumn{5}{|c|}{ Modelling Keywords } \\
\hline applied model & CGE & comput* & dynamic \\
\hline economy wide & equilibrium & forward* model & general model \\
\hline global model & international model & mathemat* & multi commodity \\
\hline multi region* & multi sector* & partial model & static \\
\hline \multicolumn{4}{|c|}{ Doha Keywords } \\
\hline doha agric* market* & doha develop* & doha market* trad* & doha non trade \\
\hline doha domestic support & export subsid* & doha trade distort* & \\
\hline doha fair trad* & doha food security & doha market access & \\
\hline
\end{tabular}

Quelle: Eigene Zusammenstellung. 


\section{Studien des Literatursamples: Publikationsjahr, Autor(en). Titel, Er-} scheinungsort:

(230 Studien, wovon 110 Studien im Meta-Datensatz verwendet wurden. Diese Studien sind durch „*“" gekennzeichnet):

\section{6}

Abler, D., Beghin, J., Blandford, D., Elobeid, A., 2006. "U.S. Sugar Policy Options and Their Consequences under NAFTA and Doha."Center for Agricultural and Rural Development, lowa State University, Working Paper 06-WP 424.

* Anderson, K., Valenzuela, E., 2006. "The World Trade Organization's Doha Cotton Initiative: A Tale of Two Issues." World Bank Policy Research Working Paper 3918.

Anderson, K., Valenzuela, E., Jackson, L., 2006. "GM Cotton Adoption, Recent and Prospective: A Global CGE Analysis of Economic Impacts." Centre for Economic Policy Research: Discussion paper 5568.

* Decreux, Y., Fontagné, L., 2006. "A Quantitative Assessment of the Outcome of the Doha Development Agenda." CEPII, Working Paper No 2006-10.

* Diaz-Bonilla, E., Diao, X., Robinson, S., 2006. "Thinking Inside the Boxes: Protection in the Development and Food Security Boxes versus Investments in the Green Box." In: Diaz-Bonilla, E. et al., eds. CAB International 2006: WTO Negotiations and Agricultural Trade Liberalization: the Effects of Developed Countries'Policies on Developing Countries.

* Frandsen, S., Jensen, H., Lind, K., Melgaard, P., Yu, W., 2006. "Assessing the Harbinson Draft on Modalities in the WTO Agricultural Negotiations." In: Diaz-Bonilla, E. et al., eds. CAB International 2006: WTO Negotiations and Agricultural Trade Liberalization: the Effects of Developed Countries'Policies on Developing Countries.

* Hertel, T., Keeney, R., 2006. "What Is at Stake: The Relative Importance of Import Barriers, Export Subsidies, and Domestic Support." In: Anderson, K. and Martin, W.: Agricultural Policy Reform and the Doha Development Agenda.

Hertel, T., Keeney, R., Ivanic, M., Winters, L., 2006. "Distributional Effects of WTO Agricultural Reforms in Rich and Poor Countries." GTAP Working Paper No. 34.

* Jha, V., 2006. "India and the Doha Work Programme." UNCTAD - Ministry of Commerce, Government of India - DFID Project Strategies and Preparedness for Trade and Globalisation in India, MacMillan India LTD.

* Lodefalk, M., and Kinnman, S., 2006. "Economic Implications of the Doha Round." Kommerskollegium- Swedish National Board of Trade, Governmental Report, Stockholm.

* Matthews, A., Walsh, K., 2006. "The Doha Development Agenda: Mixed Prospects for Developing Countries." University of Dublin - Department of Economics IIIS Discussion Paper No. 157.

Vanzetti, D., Peters, R., 2006. "Another Look at the WTO Negotiations on Agriculture." presented at the 2005 IATRC Summer Meeting Seville, Spain, June 19-21.

*Yu,W., 2006.“ Improving Agricultural Market Access for African LDCs: Deepening, Widening, Broadening and Strengthening Trade Preferences." Contributed paper prepared for presentation at the International Association of Agricultural Economists Conference, Gold Coast, Australia, August 12-18, 2006.

* Yu,W., Jensen, T., 2006. "Is the Everything But Arms Initiative the Way to go for Leastdeveloped Countries in the WTO Negotiations?" In: Diaz-Bonilla, E. et al., eds. CAB 
International 2006: WTO Negotiations and Agricultural Trade Liberalization: the Effects of Developed Countries'Policies on Developing Countries.

\section{5}

*Anderson, K., Martin, W., 2005. "Agricultural Trade Reform and the Doha Development Agenda." World Bank Policy Research Working Paper 3607.

* Anderson, K., Martin, W., van der Mensbrugghe, D., 2005. "Distortions to World Trade: Impacts on Agricultural Markets and Farm Incomes." World Bank Policy Research Working Paper 3736.

* Anderson, K., Martin, W., van der Mensbrugghe, D., 2005. "Doha Merchandise Trade Reform: What's at Stake for Developing Countries?" CEPR Discussion Paper.

Anderson, K., Martin, W., van der Mensbrugghe, D., 2005. "Market and Welfare Implication of Doha Reform Scenarios." Paper presented at the 8th Annual Conference on Global Economic Analysis, Lübeck, Germany, June 9-11, 2005, http:// www.gtap.agecon.purdue.edu/events/Conferences/2005/default.asp.

* Anderson, K., Martin, W., van der Mensbrugghe, D., 2005. "Would Multilateral Trade Reform Benefit Sub-Saharan Africans?“ C.E.P.R. Discussion Papers: 5049.

* Annabi, N., Khondker, B., Raihan, S., Cockburn, J., Decaluwe, B., 2005. "Implications of WTO Agreements and Domestic Trade Policy Reforms for Poverty in Bangladesh: Short vs. Long Run.“ MPIA Working Paper 02.

* Antimiani, A., Conforti, P., Salvatici, L., 2005. "Alternative Market Access Scenarios in the Agricultural Trade Negotiations of the Doha Round.“ The Estey Centre Journal of International Law and Trade Policy 6(2): 274-298.

* Bchir, M., Fontagné, L., Jean, S., 2005. "From Bound Duties to Actual Protection: Industrial Liberalization in the Doha Round." CEPII, Working Paper No. 2004-22.

* Brown, D., Kiyota, K., Stern, R, 2005. "Computational Analysis of the U.S. FTAs with Central America, Australia, and Morocco." Research Seminar in International Economics, Gerald R. Ford School of Public Policy, The University of Michigan Ann Arbor, Discussion Paper No. 527.

Bureau, J., Jean, S., Matthews, A., 2005. "The Consequences of Agricultural Trade Liberalization for Developing Countries: Distinguishing Between Genuine Benefits and False Hopes." CEPII, Working Paper No 2005-13.

* Chiang, F., 2005. "A Study of the Impact of Direct Trade on Taiwan's Fishery Sector with Special Reference to the Effect of China's WTO Accession " Agricultural Economics 33, no. 1 (2005): 67-77.

* Daude, S., 2005. "Agricultural Trade Liberalization in the WTO and its Poverty Implications: A Study of Rural Households in Northern Vietnam." Frankfurt am Main [u.a.] : Lang, c2005.

* Emini, C., Cockburn, J., Decaluwe, B., 2005. "The Poverty Impacts of the Doha Round in Cameroon: The Role of Tax Policy." MPIA Working Paper 04.

* Francois, J., 2005. "Trade Liberalization in the Doha Development Round." Economic policy (2005): 351-391.

Francois, J., Martin, W., Manole, V., 2005. "Choosing Formulas for Market Access Negotations: Efficiency and Market Access Considerations.“ In: Jayasuriya, S., ed., Trade Policy Reforms and Development: Essays in Honour of Professor Peter Lloyd, Vol. 2, Edward Elgar.

* Giblin, T., Mathews, A., 2005. "Global and EU Agricultural Trade Reform: What is in it for Tanzania, Uganda and Sub-Saharan Africa?.“ IIIS Discussion Paper No. 74. 
Ianchovichina, E., 2005. "Duty Drawbacks, Competitiveness, and Growth: Are Duty Drawbacks Worth the Hassle?" World Bank: Policy Research Working Paper 3498.

Ianchovichina, E., Walmsley, T., 2005. "Impact of China's WTO Accession on East Asia." Contemporary Economic Policy 23 (2), 2005, 261-277.

Jensen, J., Rutherford, T., 2005. "Telecommunications Reform within Russia's Accession to the World Trade Organization."

* Kerkelä, L., Lehtonen, H., Niemi, J., 2005. "The Impacts of WTO Expcrt Subsidy Abolition on the Agri-food Industry in the EU: A Preliminary Assessment." VATT Discussion Papers 375.

Kuhn, A., Nivyevs'kiy, O., 2005. "Evaluating the Ukrainian Oilseed Export Tax." Institute for Economic Research and Policy Consulting, Working Paper No. 29.

Limão, N., Olarreaga, M., 2005. "Trade Preferences to Small Developing Countries and the Welfare Costs of Lost Multilateral Liberalization." World Bank and CEPR Discussion Paper.

Pan, S., Welch, M., Mohanty, S., Fadiga, M., Ethridge, D., 2005. "Cotton in a Free Trade World." Cotton Economics Research Institute, Department of Agricultural and Applied Economics, Texas Tech University, Briefing Paper, CER-BR05-02.

Rosegrant, M., Cline, S., Li, W., Sulser, T., Valmonte-Santos, R., 2005. "Looking Ahead: Long-Term Prospects for Africa's Agricultural Development and Food Security." IFPRI Discussion Paper No. 41.

Rutherford, T., Tarr, D., Shepotylo, O., 2005. "Poverty Effects of Russia's WTO Accession: Modeling "Real" Households and Endogenous Productivity Effects.“ World Bank Policy Research Working Paper 3473, January 2005.

* Tumbarello, P., 2005. "Regional Trade Integration and WTO Accession: Which is the Right Sequencing? An Application to the CIS." IMF Working Paper WP 05/94.

* Yu, W., Jensen, T., 2005. "Tariff Preferences, WTO Negotiations and the LDCs: The Case of the "Everything But Arms" Initiative." Danish Research Institute of Food Economics, Fødevareøkonomisk Institut, Working Paper.

\section{4}

* Achterbosch, T., 2004. "Trade Liberalisation under the Doha Development Agenda : Options and Consequences for Africa." The Hague: Agricultural Economics Research Inst. (LEI), 2004.

* Achterbosch, T., Hammouda, H., Osakwe, P., van Tongeren, F., 2004. "Consequences of the Doha Round Trade Reforms for Africa." Paper prepared for the 7th Annual GTAP conference on Global Economic Analysis scheduled for 17-19 June 2004 in Washington DC.

* Anderson, K, 2004. "Agricultural Trade Reform and Poverty Reduction in Developing Countries." World Bank Policy Research Working Paper 3396.

Anderson, K., Huang, J., Ianchovichina, E., 2004. "Will China's WTO Accession Worsen Farm Household Incomes?“" China Economic Review 15(4): 443-56.

* Anderson, K., Jackson, L., 2004. "Standards, Trade and Protection the Case of GMOs." CIES, School of Economics, Adelaide University.

Binfield, J., Donnellan, T., Hanrahan, K., Hart, C., Westhoff, P., 2004. "CAP Reform and the WTO: Potential Impacts on EU Agriculture." Selected Paper prepared for presentation at the American Agricultural Economics Association Annual Meeting, Denver, Colorado, July 1-4, 2004. 
* Bouët, A., Bureau, J., Decreux, Y., Jean, S., 2004. "Multilateral Agricultural Trade Liberalization: The Contrasting Fortunes of Developing Countries in the Doha Round.“ CEPII, Working Paper No 2004-18.

Breuss, F., 2004. "WTO Dispute Settlement: An Economic Analysis of four EU-US Mini Trade Wars." WIFO, Wien.

Burfisher, M., 2004. "U.S. Agriculture and the Free Trade Area of the Americas." Market and Trade Division, Economic Research Service, U.S. Department of Agriculture. Agricultural Economic Report No. 827.

Chang, H., de Gorter, H., 2004. "Tariff Rate Import Quotas, Domestic Market Structure and Agricultural Support Program: The Case of Taiwanese Rice Import.“ Selected Paper of the 2004 Annual Meeting, June 30-July 2, 2004, Honolulu, Hawaii.

Chiang, F., Sun, C., Lin, C., 2004. "The Impact of Taiwan's WTO Entry on Its Domestic Agriculture Sector." Review of Urban and Regional Development Studies 16(1): 1-13.

* Conforti, P., Salvatici, L., 2004. "Agricultural Trade Liberalization in the Doha Round: Alternative Scenarios and Strategic Interactions between Developed and Developing Countries." FAO Commodity and Trade Policy Research Working Paper No. 10.

* Conforti, P., Velazquez, B., 2004. "The Effects of Alternative Proposals for Agricultural Export Subsidies in the Current WTO Round." Estey Centre Journal of International Law and Trade Policy 5(1): 11-42.

Elobeid, A., Beghin, J., 2004. "Multilateral Trade and Agricultural Policy Reforms in Sugar Markets." Center for Agricultural and Rural Development, Iowa State University, Working Paper 04-WP 356.

* Fugazza, M., Vanzetti, D., 2004. "A South-South Survival Strategy: The Potential for Trade among Developing Countries." European Trade Study Group 6th Annual Conference, Nottingham, 9-11 September 2004.

Gohin, A., Moschini, G., 2004. "Evaluating the Market and Welfare Impacts of Agricultural Policies in Developed Countries: Comparison of Partial and General Equilibrium Measures." Paper prepared for the International Conference on Policy Modeling (EcoMod2004), Paris, June 30 - July 2, 2004.

Hoekman, B., Ng, F., Olarreaga, M., 2004. "Agricultural Tariffs or Subsidies: Which Are More Important for Developing Economies?" The World Bank Economic Review 18(2): 175-204.

Ianchovichina, E., 2004. "Trade Policy Analysis in the Presence of Duty Drawbacks." Journal of Policy Modeling 26(3): 353-71.

Jensen, J., Rutherford, T., 2004. "The Impact of Liberalizing Barriers to Foreign Direct Investment in Services the Case of Russian Accession to the World Trade Organization.“ World Bank, Washington, D.C., http://www.econ.worldbank.org/ view.php?type $=5 \&$ id $=38213$.

* Keeney, R., Hertel, T., 2004. "The WTO Doha Development Round and OECD Agricultural Policy." Selected Paper prepared for presentation at the Southern Agricultural Economics Association Annual Tulsa, Oklahoma, February 18, 2004.

Konan, D., Assche, A., 2004. "Assessing the Benefits of Partial Telecommunications Liberalization to Tunisia." University of Hawaii at Manoa, Department of Economics, Working Paper.

- Lips, M., Rieder, P., 2004. "Potential Impact of the WTO Doha Round on Swiss Agriculture." Swiss Journal of Economics and Statistics 140(4): 569-83.

MacLaren, D., Liu, X., 2004. "A Potential Preferential Trade Agreement Involving China, Japan and Korea and its Implications for Agricultural Trade." Contributed Paper 
presented at the Australian Agriculture and Ressource Society 48th Annual Conference Melbourne.

Mohanty, S., Pohit, S., Roy, S., 2004. "Towards Formation of Close Economic Cooperation among Asian Countries." Research and Information System for the Non-Aligned and Other Developing Countries RIS-DP 78.

Morley, S., Piñeiro, V., 2004. "The Effect of WTO and FTAA on Agriculture and the Rural Sector in Latin America." Development Strategy and Governance Division International Food Policy Research Institute, Washington, D.C., DSGD Discussion Paper No. 3.

* Peters, R., Vanzetti, D., 2004. "Shifting Sands: Searching for a Compromise in the WTO Negotiations on Agriculture." UNCTAD Discussion Paper. Policy Issues in International Trade and Commodities, Study Series No. 23.

Rees, L., Tyers, R., 2004. "Trade Reform in the Short Run: China's WTO Accession." Journal of Asian Economics 15(1): 1-31.

Tian, W., Zhou, Z., 2004. "China's Agricultural Trade Prospects and International Trade Reforms." Contributed Paper presented at the Australian Agriculture and Ressource Society 48th Annual Conference Melbourne.

* van der Mensbrugghe, D., Beghin, J., 2004. "Global Agricultural Liberalization: An InDepth Assessment of What is at Stake." CARD Working Paper 04-WP 370.

\section{3}

Acosta, L., Kagatsume, M., 2003. "ASEAN Rice Sector in the WTO: Analysis of Demand and Supply in a Liberalized Trade Environment.“. ASEAN-Economic-Bulletin 20(3): 233-243.

Alekseev, A., Tourdyeva, N., Yudaeva, K., 2003. "Estimation of the Russia's Trade Policy Options with the Help of the Computable General Equilibrium Model." Centre for Economic and Financial Research (CEFIR), Working Paper (Draft).

Anderson, K., 2003. "Trade Liberalization, Agriculture, and Poverty in Low-income Countries." CEPR, and School of Economics and Centre for International Economic Studies, University of Adelaide, Australia. Discussion Paper.

* Beghin, J., Diop, N., Matthey, H., Sewadeh, M., 2003. “Groundnut Trade Liberalization: A South-South Debate?" Center for Agricultural and Rural Development, Iowa State University, Working Paper 03-WP 347.

* Beghin, J., Diop, N., Matthey, H., Sewadeh, M., 2003. "The Impact of Groundnut Trade Liberalization: Implication for the Doha Round." Paper prepared for presentation at the American Agricultural Economics Association Annual Meeting, Montreal, Canada, July 27-30, 2003.

* Beghin, J., Matthey, H., 2003. "Modeling World Peanut Product Markets: A Tool for Agricultural Trade Policy Analysis." Center for Agricultural and Rural Development, Iowa State University, Working Paper 03-WP 332.

Binfield, J., Donnellan, T., Hanrahan, K., Westhoff, P., 2003. "The Luxembourg CAP Reform Agreement: Analysis of the Impact on EU and Irish Agriculture." FAPRI Ireland Partnership. Project Report.

* Bout, A., Bureau, J., Decreux, Y., Jean, S., 2003. "Is Northern Agricultural Liberalization Beneficial for Developing Countries." Discussion Paper CEPII, website download from http://www.ecomod.net/conferences/ecomod2003/ecomod2003/papers/Bouet Agricultural.pdf . 
* Bradford, S., 2003. "Non-Tariff Barriers in Rich Countries: Quantifying Them, Identifying Them, and Assessing Their Impacts." Brigham Young University, Discussion Paper.

* Brown, D., Deardorff, A., Stern, R., 2003. "Developing Countries' Stake in the Doha Round.“ The University of Michigan, Research Seminar in International Economics, Discussion Paper No. 495.

Brown, D., Deardorff, A., Stern, R., 2003. "Multilateral, Regional and Bilateral TradePolicy Options for the United States and Japan." World-Economy 26(6): 803-28.

* Cernat, L., Laird, S., Monge-Roffaraello, L., Turrini, A., 2003. "The EU's Everything But Arms Initiative and the Least-developed Countries." In Stern, R., ed., Issues and Options for U.S.-Japan Trade Policies. University of Michigan Press, Ann Arbor.

Diao, X., 2003. "China's WTO Accession: Impacts on Regional Agricultural Income: A Multi-Region, General Equilibrium Analysis." Journal of comparative economics 31(2): 332-351.

Diao, $X$., 2003. "Regional and National Perspectives of China's Integration into the WTO: A CGE Inquiry with Emphasis on the Agricultural Sector." Review of urban \& regional development studies 15(2): 84-105.

* Elbehri, A., Hertel, T., Martin, W., 2003. "Estimating the Impact of WTO and Domestic Reforms on the Indian Cotton and Textile Sectors: A General-Equilibrium Approach ." Review of Development Economics 7(3): 343-359.

European Commission, 2003. "Mid-Term Review Proposal Impact Analysis with the CAPRI Modelling System." Presented at the Policy Research Symposium.

Fang, C., Babcock, B., 2003. "China's Cotton Policy and the Impact of China's WTO Accession and BT Cotton Adoption on the Chinese and U.S. Cotton Sectors." CARD Working Paper 03-WP 322.

Felloni, F., 2003. "Trade Policy, Biotechnology and Grain Self-sufficiency in China." Agricultural Economics 28(3): 173-86.

* Flasbarth, A., Lips, M., 2003. "Effects of a Humanitarian WTO Social Clause on Welfare and North-South Trade Flows." Discussion paper no. 2003-03, University of St Gallen, Department of Economics.

* Fontagné, L., Guérin, J., Jean, S., 2003. "Market Access Liberalisation in the Doha Round: Scenarios and Assessment." Working Paper No 2003-12.

Francois, J., van Meijl, H., van Tongeren, F., 2003. "A Forward Looking Analysis of the Doha Round: Agriculture, Manufacturing and Services." Contributed paper presented at the International Conference Agricultural policy reform and the WTO: where are we heading? Capri (Italy), June 23-26, 2003.

* Francois, J., van Meijl, H., van Tongeren, F., 2003. "Economic Implications of Trade Liberalization under the Doha Round." CEPII research center, Working Paper.

* Francois, J., van Meijl, H., van Tongeren, F., 2003. "French Agriculture and Processed Foods in the Doha Round.“ Groupe d'Economie Mondiale de Sciences Po. Discussion Paper.

* Francois, J., van Meijl, H., van Tongeren, F., 2003. "Trade Liberalization and Developing Countries under the Doha Round." Tinbergen Institute Discussion Paper Tl 2003-060/2.

* Frandsen, S., Jensen, H., Lind, K., Melgaard, P., Yu, W., 2003. "Note on the Harbinson Draft on Modalities in the WTO Agriculture Negotiations." Agricultural Policy Research Division, Danish Research Institute of Food Economics, Discussion Paper. 
Ghosh, M., Perroni, C., Whalley, J., 2003. "Developing Country Benefits from MFN Relative to Regional/Bilateral Trade Agreements." Review of International Economics 11(4): 712-28.

* Gilbert, J., Wahl, T., 2003. "Labor Market Distortions and China's WTO Accession Package: An Applied General Equilibrium Assessment.“ Journal of Comparative Economics 31(4): 774-794.

* Harrison, G., Rutherford, T., Tarr, D., Gurgel, A., 2003. "Trade Policy and Poverty Reduction in Brazil." Central Bank of Chile Working Papers 276.

Hilaire, A., Yang, Y., 2003. "The United States and the New Regionalism/Bilateralism." IMF Working Paper, Policy Development and Review Department.

* Ianchovichina, E., Anderson, K., Huang, J., 2003. "Long-Run Impacts of China's WTO Accession on Farm-Nonfarm Income Inequality and Rural Poverty.“ The World Bank, Policy Research Working Paper Series: 3052.

Jansson, T., Wieck, C., Dominguez, I., Britz, W., 2003. "Auswirkungen der Mid-Term Review Beschlüsse auf die Landnutzung in europäischen Regionen und Betrieben eine quantitative Analyse mit dem CAPRI Modellsyste." Paper presented at the Gewisola-Tagung 2003, Hohenheim, September 29 - October 1, 2003.

Kruse, J., 2003. "Implications of the 2002 U.S. Farm Act for World Agriculture." Paper presented at the: Policy Disputes Information Consortium's Ninth Agricultural and Food Policy Information Workshop "Farm Policy Developments and Tensions with NAFTA", Montreal, Canada, April 23-26, 2003.

Laens, S., Terra, M., 2003. "Integration of the Americas: Welfare Effects and Options for the MERCOSUR." Departamento de Economía, Facultad de Ciencias Sociales, Project Report.

* Laird, S., Cernat, L., Turrini, A., 2003. "Back to Basics: Market Issues in the Doha Agenda." UNCTAD, United Nations Publications, UNCTAD/DITC/TAB/Misc.9.

* Laird, S., de Cordoba, S., Vanzetti, D., 2003. "Market Access Proposals for Nonagricultural Products." CREDIT Research Paper No. 03/08.

* Lippoldt, D., Kowalski, P., 2003. "The Doha Development Agenda: Welfare Gains From Further Multilateral Trade Liberalisation With Respect to Tariffs." OECD,TD/TC/WP(2003)10/FINAL, www.oecd.org/trade.

* Lips, M., 2003. "The CAP Mid Term Review and the WTO Doha Round; Analyses for the Netherlands, EU and Accession Countries." LEI Report.

* Lips, M., Tabeau, A., van Tongeren, F., 2003. "Multilateral and Regional Trade Agreements: Options for Bangladesh." 6th Conference on Global Economic Analysis, The Hague, The Netherlands, June 12-14, 2003.

* Lips, M., Tabeau, A., van Tongeren, F., Ahmen, N., Herok, C., 2003. "Textile and Wearing Apparel Sector Liberalization - Consequences for the Bangladesh Economy." 6th Conference on Global Economic Analysis, The Hague, The Netherlands, June 12 - 14, 2003.

* Mai, Y., 2003. "Effects of Reducting Tariffs and Endogenous Productivity Growth." Centre for Policy Studies, Monash University, General Working Paper No. G-139.

* Organisation for Economic Co-operation and Development, 2003. "Agricultural Trade and Poverty: Making Policy Analysis Count." Washington, D.C., 2003; 328.

Organisation for Economic Co-operation and Development, 2003. "Quantitative Assessment of the Benefits of Trade Facilitation." OECD, TD/TC/WP(2003) 31/FINAL, www.oecd.org/trade. 
* Poonyth, D., Sharma, R., 2003. "The Impact of the WTO Negotiating Modalities in the Areas of Domestic Support, Market Access and Export Competition on Developing Countries: Results from ATPSM." Contributed paper presented at the International Conference Agricultural policy reform and the WTO: where are we heading? Capri (Italy), June 23-26, 2003.

Pustovit, N., Schmitz, P., 2003. "Impact of Agricultural Protection in OECD-Countries on South African Agriculture." Contributed paper selected for presentation at the 25th International Conference of Agricultural Economists, August 16-22, 2003, Durban, South Africa.

* Rae, A., Strutt, A., 2003. "The Current Round of Agricultural Trade Negotiations: Should We Bother about Domestic Support?.“ Estey Centre Journal of International Law and Trade Policy 4(2): 98-122.

Ravallion, M., Chen, S., 2003. "Household Welfare Impacts of China's Accession to the World Trade Organization.“ The World Bank, Policy Research Working Paper Series: 3040.

* Redmond, W., 2003. "A Quantification of Policy Reform: An Application to the Uruguay Round Negotiations on Agriculture.“ Journal of Policy Modeling 25(9): 893-910.

* Rege, S., 2003. "Applied General Equilibrium Analysis of India's Tax and Trade Policy.“ Aldershot, U.K. and Burlington, Vt.: Ashgate, 2003; xiv, 238.

* Terra, M., 2003. "Trade Liberalization in Latin American Countries and the Agreement on Textiles and Clothing in the WTO.“ Economie-Internationale 2nd-3rd Trimester 2003; 0(94-95): 137-54.

*Vanzetti, D., Peters, R., 2003. "An Analysis of the Proposals by the World Trade Organization, the United States and the European Union on Agricultural Reform.“ Papers presented at the "High-level Regional Policy Dialogue on the WTO Negotiating Agenda in Preparation for Cancún" and the "Regional Seminar on Facilitating the Accession of ESCAP Members to WTO through Regional Cooperation" Bangkok, 10-13 June 2003.

*Vanzetti, D., Peters, R., 2003. "Making Sense of Agricultural Trade Policy Reform." IAAEConference Durban.

Wang, Z., 2003. "The Impact of China's WTO Accession on Patterns of World Trade." Journal of Policy Modeling 25(1): 1-41.

Wang, Z., 2003. "WTO Accession, the "Greater China" Free-trade Area, and Economic Integration Across the Taiwan Strait." China economic review 14(3): 316-349.

Weber, G., 2003. "Russia's and Kazakhstan's Agro-food Sectors under Liberalized Agricultural Trade: A Case for National Product Differentiation." Economic Systems 27(4): 391-413.

* Wieck, C., Dominguez, I., Britz, W., 2003. "New Challenges for the European Agriculture: Modelling Agricultural Reform Under the New WTO Proposals." Contributed paper presented at the International Conference Agricultural policy reform and the WTO: where are we heading? Capri (Italy), June 23-26, 2003.

Witzke, H., Kuhn, A., 2003. "Assessing Reform Options for the Sugar Common Market Organisation - Quantitative Analyses with Interlinked Models.“ Presented at 43. Jahrestagung der Gesellschaft für Wirtschafts- und Sozialwissenschaften des Landbaues e.V. vom 29. September bis I. Oktober 2003 in Stuttgart-Hohenheim.

* Zhai, F., Hertel, T., Wang, Z., 2003. "Labor Market Distortions, Rural-Urban Inequality and the Opening of China's Economy." GTAP Working Paper No. 27. 
2002

* Agbenyegah, C., 2002. "The Impacts of Trade Liberalisation on the World Rice Market." Master of Commerce and Management. Commerce Division Lincoln University Canterbury.

Anderson, K., 2002. "Agricultural Trade and Rural Poverty Reduction: Market Access." CEPR, and School of Economics and Centre for International Economic Studies, University of Adelaide.

* Beghin, J., Roland-Holst, D., van der Mensbrugghe, D., 2002. "Global Agricultural Trade and the Doha Round: What are the Stakes for North and South?." Paper presented at the OECD - World Bank Forum on Agricultural Trade Reform, Adjustment, and Poverty, in Paris, May 23-24, 2002 and the Fifth Conference on Global Economic Analysis, Taipei, June 5-7, 2002.

Bouamra-Mechemache, Z., 2002. "EU Dairy Policy Reform and Future WTO Negotiations: A Spatial Equilibrium Analysis." Journal of agricultural economics 53(2): 233-257.

* Brown, D., Deardorff, A., Stern, R., 2002. "Computational Analysis of Multilateral Trade Liberalization in the Uruguay Round and Doha Development Round." Research Seminar in International Economics, Gerald R. Ford School of Public Policy, The University of Michigan Ann Arbor, Discussion Paper No. 489.

Conforti, P., 2002. "Assessing the Effects of the Reform of the EU Rice Common Market Organisation." Istituto Nazionale di Economia Agraria, English abridged version of Working Paper n.6.

* Conforti, P., de Filippis, F., Salvatici, L., 2002. "The Mid-Term Review of the Common Agricultural Policy: Assessing the Effects of the Commission Proposals." INEA, The National Institute of Agricultural Economics, Working Paper n.18.

Diao, X., Diaz-Bonilla, E., Robinson, S., 2002. "Scenarios for Trade Integration in the Americas." Trade and Macroeconomics Division, International Food Policy Research Institute, Washington, D.C., U.S.A. Discussion Paper No. 90.

Diao, X., Fan, S., Zhang, X., 2002. How China's WTO Accession Affects Rural Economy in the Less-developed Regions: A Multi-Region, General Equilibrium Analysis. Washington, DC.

* Dimaranan, B., Hertel, T., Martin, W., 2002. "Potential Gains from Post-UR Trade Reform: Impacts on Developing Countries." presented at the summer symposium of the International Agricultural Trade Research Consortium on: "The Developing Countries, Agricultural Trade and the WTO," Whistler, British Columbia, Canada, June 16-17, 2002.

Doi, M., Tiwari, P., Kawakami, T., 2002. "Trade and Factor Market Reforms in China." Labour 16(3): 537-555.

Haddad, E., Domingues, E., Perobelli, F., 2002. "Short-run Regional Effects of Alternative Strategies for Economic Integration: The Case of Brazil.“" Paper prepared for the 41 st Congress of the European Regional Science Association.

* Hoekman, B., Ng, F., Olarreaga, M., 2002. "Reducing Agricultural Tariffs Versus Domestic Support: What's More Important for Developing Countries?.“ The World Bank, Policy Research Working Paper Series: 2918.

Ko, J., 2002. "Eastern Enlargement of the EU and Analysis of its Economic Impact Using a CGE Model." International Conference on "EU Eastern Enlargement Coming Soon. Strategy of Membership", University of Gdansk, Sopot, Poland, 19-20 April 2002.

Kuhn, A., Wehrheim, P., 2002. "Agricultural Trade Diversion Due to EU Eastern Enlargement a Quantitative Analysis Based on a Partial Equilibrium World Trade 
Model (WATSIM).“ Paper presented at 42. Jahrestagung der GeWiSoLa vom 30.9. 02.10 .2001 in Halle/Saale.

* Laird, S., Peters, R., Vanzetti, D., 2002. "Southern Discomfort: Agricultural Policies, Trade and Poverty." Centre for Research in Economic Development and International Trade, University of Nottingham, 04/02.

MAF International Policy, 2002. "Rewinding the Uruguay Round of Multilateral Trade Negotiations: How has New Zealand Agriculture Benefited?.“ New Zealand Ministry of Agriculture and Forestry, Report, http://www.maf.govt.nz/mafnet/rural$\mathrm{nz} /$ profitability-and-economics/trends/uruguay-trade-round-negotiations/httoc.htm.

Nielsen, C., 2002. "Vietnam's Rice Policy: Recent Reforms and Future Opportunities." Danish Research Institute of Food Economics, Fødevareøkonomisk Institut, Working Paper 8.

$O E C D, 2002$. "Agriculture and Trade Liberalization. Extending the Uruguay Round Agreement." OECD Paris. Monograph.

* Shakur, S., 2002. How Comprehensive will be the Doha Round?: Experiments with Agricultural and Non-agricultural Reforms. Palmerston North.

* Shantong, L., Zhai, F., 2002. "China's WTO Accession and Implications for Its Regional Economies." Economie-Internationale 4th Trimester 2002; 0(92): 67-102.

Stone, S., Matysek, A., Dolling, A., 2002. "Modelling Possible Impacts of GM Crops on Australian Trade." Productivity Commission Staff Research Paper, Melbourne, October.

Stout, J., Leetmaa, S., Normile, M., 2002. "Evaluating EU Agricultural Policy Reform Using the EU WTO Model." Economic Research Service, USDA, Washington, DC.

Tiwari, P., Kawakami, T., Doi, M., 2002. "Dual Labor Markets and Trade Reform in China." Journal of Policy Reform 5(2): 101-13.

* Witzke, H., 2002. "Impact Analysis of the European Commission's Proposal under the MidTerm Review of the CAP Using the CAPSIM Model." EuroCARE University of Bonn, MTR Impact assessment, Report.

Witzke, H., Heckelei, T., 2002. "EU Sugar Policy Reform: Quota Reduction and Devaluation." Selected Paper American Agricultural Economics Association, Long Beach, July $28-31,2002$.

Zhai, F., Wang, Z., 2002. "WTO Accession, Rural Labour Migration and Urban Unemployment in China." Urban Studies 39(12): 2199-2217.

\section{1}

Abler, D., Blandford, D., Bohman, M., Dixit, P., Stout, J., 2001. "Development of and Initial Results from the ERS/Penn State WTO Model." Paper prepared for presentation at the International Agricultural Trade Research Consortium meeting, Washington DC, May 18-20, 2001.

Anania, G., 2001. "Modeling the GATT "Agreement on agriculture". Assessing the Compatibility of EU "Agenda 2000" with GATT Commitments for Wheat." In Heckelei, T., Witzke, H., Henrichsmeyer, eds., W. Agricultural Sector Modelling and Policy Information Systems. Wissenschaftsverlag, Vauk Kiel, 299-308.

Beghin, J., Bureau, J., Park, S., 2001. "Food Security and Agricultural Protection in South Korea." Working Paper 01-WP 284.

Beghin, J., El Osta, B., Cherlow, J., Mohanty, S., 2001. "The Cost of the U.S. Sugar Program Revisited.“ Working Paper 01-WP 273, CARD, lowa State University. 
Bouamra-Mechemache, Z., Chavas, J., Cox, T., Requillart, V., 2001. "Price Discrimination and EU Dairy Policy: An Economic Evaluation of Policy Options." Cahier de Recherche, INRA, Working Paper 11.

Breuss, F., 2001. "WTO Dispute Settlement from an Economic Perspective: More Failure than Success?." Forschungsinst. für Europafragen, Wien.

Brown, D., Deardorff, A., Stern, R., 2001. "CGE Modeling and Analysis of Multilateral and Regional Negotiating Options." Research Seminar in International Economics, Gerald R. Ford School of Public Policy, The University of Michigan Ann Arbor, Discussion Paper No. 527.

Burfisher, M., 2001. "Agricultural Policy Reform in the WTO - The Road Ahead." Market and Trade Economics Division, Economic Research Service, U.S. Department of Agriculture. Agricultural Economic Report No. 802.

Cameron, R., Loukine, K., 2001. "Canada - European Union Trade and Investment Relations - The Impact of Tariff Elimination." Study prepared for the review of the interdepartmental steering committee on December 14, 2000.

* Chow, P., Tuan, F., Wang, Z., 2001. "The Impacts of WTO Membership on Economic/Trade Relations among Three Chinese Economies: China, Hong Kong and Taiwan." Pacific Economic Review 6(3): 419-44.

* Diao, X., Somwaru, A., Roe, T., 2001. "A Global Analysis of Agricultural Reform in WTO Member Countries." In Burfisher, M., ed., Agricultural Policy Reform-The Road Ahead. AER 802 Economic Research Service, USDA.

* Elbehri, A., Leetmaa, S., 2001. "How Significant are Export Subsidies to Agricultural Trade? Trade and Welfare Implications of Global Reforms." Paper presented at the Annual Meeting of the American Agricultural Economics Association.

* Fan, M., Zheng, Y., 2001. "The Impact of China's Trade Liberalization for WTO Accession - A Computable General Equilibrium Analysis.“ Paper presented at the Greater China and WTO International Conference, Hong Kong.

* Francois, J., 2001. "Modelling the Impact of WTO Negotiations on EU Agriculture: An Application of the GTAP Model.“ In van Tongeren, F., van Meijl, H., eds., European policy issues in a global trade analysis framework. Agricultural Economics Research Institute (LEI), The Hague, Report 6.01.07.

* Francois, J., 2001. "The Next WTO Round: North-South Stakes in New Market Access Negotiations." Centre for International Economic Studies , Adelaide. Tinbergen Institute, Amsterdam and Rotterdam. Discussion Paper.

Frandsen, S., Jensen, H., 2001. "Economic Impacts of the Enlargement of the European Union; Analysing the Importance of Direct Payments." In van Tongeren, F., van Meijl, H., eds., European policy issues in a global trade analysis framework. Agricultural Economics Research Institute (LEI), The Hague, Report 6.01.06.

Fuller, F., Beghin, J., de Cara, S., Fabiosa, J., Fang, C., 2001. "China's Accession to the WTO: What is at Stake for Agricultural Markets?.“ Working Paper 01-WP 276, CARD, lowa State University.

Hertel, T., Martin, W., 2001. "Second-Best Linkages and the Gains from Global Reform of Manufactures Trade." Review of International Economics 9(2): 215-32.

Hertel, T., Walmsley, T., Itakura, K., 2001. "Dynamic Effects of the "New Age" Free Trade Agreement between Japan and Singapore." GTAP working paper.

*Hosoe, N., 2001. "A General Equilibrium Analysis of Jordan's Trade Liberalization." Journal of Policy Modeling 23(6): S.595-600. 
Ianchovichina, E., Martin, W., Fukase, E., 2001. "Assessing the Implications of Merchandise Trade Liberalization in China's Accession to WTO.“ Paper presented at the Greater China and WTO International Conference, Hong Kong.

Leetmaa, S., 2001. "Effects of Eliminating EU Export Subsidies." In Burfisher, M., ed., Agricultural Policy Reform-The Road Ahead. AER 802 Economic Research Service, USDA.

* Li, X., Lejour, A., 2001. "The Sectoral Impact of China's Access to the WTO - A Dynamic CGE Analysis." Paper presented at the Greater China and WTO International Conference, Hong Kong.

Lloyd, P., Zhang, X., 2001. "Models of the Chinese Economy." Cheltenham, U.K. and Northampton, Mass.: Elgar; distributed by American International Distribution Corporation, Williston, Vt., 2001 ; xvii, 309.

Rae, A., 2001. "The Role of Grasslands in World Food Trade: Some Projected Impacts of Future Trade Policy Reforms." Presented at the XIX International Grassland Congress, Sao Pedro, Sao Paulo, Brazil, 11-21 February 2001.

Scollay, R., Gilbert, J., 2001. "An Integrated Approach to Agricultural Trade and Development Issues: Exploring the Welfare and Distribution Issues." Policy Issues in International Trade and Commodities, Study Series No. 11.

"Sohinger, J., 2001. "General Equilibrium Analysis of Croatia's Accession to the World Trade Organization." Croatian National Bank, Public Relations and Publishing Department, Working Paper.

Srinivasan, $P$., Jha, S., 2001. "Liberalized Trade and Domestic Price Stability: The Case of Rice and Wheat in India." Journal of Development Economics 65(2): 417-41.

* Sun, C., Chiang, F., Lin, C., Lin, K., 2001. "A Study of the Impacts of Tariff Reductions on Agriculture, Livestock, and Fishery Sectors in Taiwan - an Application of SingleCountry Multiple-Sector CGE Model.“ American Agricultural Economics Association.

* Tongzon, J., 2001. "China's Membership in the World Trade Organization (WTO) and the Exports of the Developing Economies of East Asia: A Computable General Equilibrium Approach." Applied Economics 33(15): 1943-1959.

* van Meijl, H., van Tongeren, F., 2001. "Mulilateral Trade Liberalisation and Developing Countries: A North-South Perspective on Agriculture and Processing Sectors." Paper prepared for the Fourth Annual Conference on Global Economic Analysis Purdue University, West Lafayette, IN June 27-29, 2001.

Verikios, G., Zhang, X., 2001. "Global Gains from Liberalising Trade in Telecommunications and Financial Services." Productivity Commission Staff, Research Paper, Ausinfo, Canberra.

*Wahl, O., 2001. "Effects of the Common Agricultural Market and Accession to the WTO on the Russian Agricultural Sector." Agricultural sector modelling and policy information systems (2001), pp.211-219.

Walmsley, T., Hertel, T., 2001. "Assessing the Impact of China's WTO Accession on Foreign Investment?.“ Paper presented at the Greater China and WTO International Conference, Hong Kong.

Wang, Z., 2001. "The Impact of China's WTO Accession on Trade and Economic Relations across the Taiwan Strait." The Economics of Transition 9(3): 743-785 .

Zhou, S., Liu, M., Banuri, T., 2001. "Country Report on Environmental Impacts of Trade Liberalization in the Chinese Cotton Sector." AERI of the Nanjing Agricultural University, Project Report (Draft). 
2000

* Anderson, K., 2000. "GMOs, Food Safety and the Environment: What Role for Trade Policy and the WTO?." CIES Policy Discussion Paper No. 0034, University of Adelaide.

Brown, D., Stern, R., 2000. "Measurement and Modeling of the Economic Effects of Trade and Investment Barriers in Services." School of Public Policy, The University of Michigan, Discussion Paper No. 453.

Chatti, R., 2000. "General Equilibrium Assessment of Trade Liberalization Effects under Cournot Oligopoly Market Structures: The Case of Tunisia." Economic Research Forum Working Paper Series, WP No. 2009.

* Dee, P., Hanslow, K., 2000. "Multilateral Liberalisation of Service Trade." Productivity Commission Staff Research Paper, Ausinfo, Canberra.

FAPRI, 2000. "FAPRI Analysis of CAP Reform in the Agenda 2000 Final Decisions." FAPRI discussion papers. www.fapri.iastate.edu.

* Felloni, F., Gilbert, J., Wahl, T., Wandschneider, P., 2000. "Self-Sufficiency and Productivity in Chinese Agriculture: Implications for China's WTO Accession." American Agricultural Economics Association.

* Freeman, F., Mélanie, J., Roberts, I., Vanzetti, D., Tielu, A., Beutre, B., 2000. "The Impact of Agricultural Trade Liberalization on Developing Countries." Study prepared for the review of the interdepartmental steering committee on December 14, 2000.

* Glismann, H., 2000. Handelsliberalisierung in der Millennium-Runde: Strategien und ihre Auswirkungen für Deutschland. Tübingen : Mohr.

Herok, C., Lotze, H., 2000. "Implications of an EU Eastern Enlargement under a New Common Agricultural Policy." Journal of Policy Modeling 22(6): 661-90.

* Hertel, T., Anderson, K., Francois, J., Martin, W., 2000. "Agriculture and Non-Agriculture Liberalization in the Millennium Round. ." CIES Policy Discussion Paper No. 276. University of Adelaide.

McDonald, S., Tsheko, O., 2000. "Scarcity and Abundance: A Study of Water and Diamonds in Botswana." Department of Economics, The University of Sheffield, Discussion Paper (Draft).

Nielsen, C., Anderson, K., 2000. "GMOs, Trade Policy, and Welfare in Rich and Poor Countries." Paper prepared for a World Bank Workshop on Standards, Regulation and Trade, to be held in Washington, D.C., 27 April 2000.

Unterschultz, J., Jeffrey, S., Quagrainie, K., 2000. "Value-adding 20 Billion by 2005: Impact at the Alberta Farm Gate." University of Alberta, Project Report 00-05.

*Walmsley, T., Hertel, T., 2000. "China's Accession to the WTO: Timing is Everything." GTAP working paper. Center for Global Trade Analyis.

Zhou, J., 2000. "Taiwan's Accession into the WTO and Trade in Services - A Computable General Equilibrium Analysis “ Cambridge, Mass. : National Bureau of Economic Reaseach.

\section{9}

Anderson, K., Hoekman, B., Strutt, A., 1999. "Agriculture and the WTO: The Next Steps." Paper prepared for the Second Annual Conference on Global Economic Analysis, Avernaes Conference Centre, Helnaes, Denmark, 20-22 June 1999.

Anderson, K., Strutt, A., 1999. "China's Economic Growth, Policy Reforms and WTO Accession: Implication for Agriculture in China and Elsewhere by 2005." Second 
Annual Conference on Global Economic Analysis, Avernaes Conference Centre, Helnaes, Denmark, 20-22 June 1999.

* Dessus, S., Fukasaku, K., Safadi, R., 1999. "Multilateral Tariff Liberalisation and the Developing Countries." OECD Development Centre Policy Brief No. 18.

Faehn, T., Grunfeld, L., 1999. "Recent Leaps towards Free Trade: The Impact on Norwegian Industry and Trade Patterns." Journal of Policy Modeling 21(6): 715-45.

* Francois, J., McDonald, B., 1999. "The Multilateral Trade Agenda: Uruguay Round Implementation and Beyond." C.E.P.R. Discussion Papers, CEPR Discussion Papers: 1533.

* Nagarajan, N., 1999. "The Millennium Round: An economic Appraisal.“ ECFIN/659/99Rev. EN Economic Papers Number 139.

Storm, S., 1999. "Foodgrain Price Stablisation in an Open Economy: A CGE Analysis of Variable Trade Levies for India." Journal of Development Studies 36(2): 136-59.

Thompson, W., Liapis, P., Sckokai, P., 1999. "Alternative Trade Mechanisms in World Dairy Markets." Presented at the Policy Research Symposium National \& Trade Dairy Policies: Implications for the Next WTO Negotiations.

Wang, Z., 1999. "The Impact of China's WTO Entry on the World Labour-intensive Export Market: A Recursive Dynamic CGE Analysis.“ The world economy 22(3): 379-405.

\section{8}

Anderson, K., Pangestu, M., 1998. "Structural Changes in a Reforming World Economy: Implications for Indonesia." Bulletin of Indonesian Economic Studies 34(3): 85-113.

* Ghosh, M., Perroni, C., Whalley, J., 1998. "The Value of MFN Treatment." National Bureau of Economic Research, Inc, NBER Working Papers: 6461.

* Mai, Y., 1998. "Trade Liberalisation within a Unilateral, Regional and Global Context.“ In China's reform and economic growth. 65-100.

Weyerbrock, S., 1998. "Reform of the European Union's Common Agricultural Policy: How to Reach GATT-Compatibility?." European economic review 42(2): 375-411.

Yang, Y., Zhong, C., 1998. "China's Textile and Clothing Exports in a Changing World Economy.“ Developing-Economies 36(1): 3-23.

\section{7}

Anderson, K., Francois, J., 1997. "Commercial Links Between Western Europe and East Asia: Retrospect and Prospects.“ C.E.P.R. Discussion Papers, CEPR Discussion Papers: 17601997.

Erwidodo, Feridhanusetyawan, T., 1997. Indonesia's Agriculture: Facing the APEC and WTO. Adelaide.

* Harrison, G., Rutherford, T., Tarr, D., 1997. "Quantifying the Uruguay Round." The Economic Journal 110(444): 1405-1430.

*Wang, Z., 1997. "China and Taiwan Access to the World Trade Organization: Implications for US Agriculture and Trade." Agricultural economics 17(2): 239-264.

\section{6}

Anderson, K., Dimaranan, B., Hertel, T., Martin, W., 1996. “Asia-Pacific Food Markets and Trade in 2005: A Global, Economy-wide Perspective." C.E.P.R. Discussion Papers, CEPR Discussion Papers: 14741996. 
Bach, C., Pearson, K., 1996. "Implementing Quotas in GTAP Using GEMPACK or How to Linearize an Inequality." GTAP Technical Paper No. 4, 1996.

Harrison, G., Rutherford, T., Tarr, D., 1996. "Economic Implications for Turkey of a Customs Union with the European Union." The World Bank, International Economics Department, International Trade Division. Policy Research Working Paper 1599.

* Pyo, H., Kim, K., Cheong, I., 1996. "Foreign Import Restrictions, WTO Commitments, and Welfare Effects: The Case of Republic of Korea." Asian Development Review 14(2): 21-43.

Taylor, J., 1996. "Development Strategy, Employment and Migration: Insights from Models.“ Organisation for economic Co-Operation and Development.

\section{5}

Clarete, R., 1995. "How Valuable Is MFN Treatment to the Philippines?.“ Philippine Review of Economics and Business 32(2): 171-95.

\section{4}

GATT, 1994. "The Results of the Uruguay Round of Multilateral Trade Negotiations Market Access for Goods and Services: Overview of the Results.“ GATT Secretary, Report, Geneva. 


\section{Anhang B: Fixed-Effects der aggregierten Regionen im Datensatz}

Die nachfolgenden Fixed-Effects sind Teil der Regressionen in Tabelle 7 und Tabelle 8. Die Bezeichnungen der Regionen sind den tatsächlichen Bezeichnungen innerhalb der Studien nachempfunden, da viele Regionen bei unterschiedlichen Bezeichnungen identisch aggregiert sind (Beispiel: Studie A „AusNZ“, Studie B „Australia\&NewZealand“. Der entsprechende Dummy für diese Region lautet ausnzl. Sofern der regionale Bezug aus einem Dummy nicht unmittelbar ersichtlich ist, handelt es sich in den meisten Fällen um Bezeichnungen, welche innerhalb der GTAP Datenbank üblich sind. Ferner werden häufig Regionen $\mathrm{zu}$ bestehenden Freihandelszonen aggregiert (Dummies „nafta“, „,efta“, etc).

Tabelle 14: Koeffizienten regionaler Aggregationen (ungewichtet).

\begin{tabular}{|c|c|c|c|c|c|c|c|c|c|c|}
\hline Stichprobe $\rightarrow$ & & Ungewi & htet mit & $B+L^{\circ}$ & & & gewich & t mit oh & e $B+L^{*}$ & \\
\hline Dummy & Koeff. & $\begin{array}{r}\text { Std. } \\
\text { Fehl. }\end{array}$ & $t-$ Wert & $\operatorname{Pr}(>\mid t))$ & & Koeff. & $\begin{array}{r}\text { Std. } \\
\text { Fehl. }\end{array}$ & $t$-Wert & $\operatorname{Pr}(>|t|)$ & \\
\hline afghanistan & 3743 & 2322 & 1,612 & 0,107 & & 5608 & 2028 & 2,765 & 0,006 & **** \\
\hline africa & 3742 & 2950 & 1,268 & 0,205 & & 3676 & 2277 & 1,615 & 0,106 & \\
\hline albania & 3770 & 2367 & 1,593 & 0,111 & & 5777 & 2063 & 2,800 & 0,005 & *** \\
\hline algeria & 3679 & 2367 & 1,554 & 0,120 & & 5686 & 2065 & 2,754 & 0,006 & *** \\
\hline andean & 5126 & 2466 & 2,078 & 0,038 & $* *$ & 5595 & 2298 & 2,435 & 0,015 & ** \\
\hline angola & 3780 & 2367 & 1,597 & 0,110 & & 5786 & 2063 & 2,805 & 0,005 & *** \\
\hline antigua & 3819 & 2442 & 1,564 & 0,118 & & 6037 & 2113 & $2,85 \pi$ & 0,004 & *** \\
\hline argentina & 4442 & 2338 & 1,900 & 0,057 & * & 5665 & 2092 & $2,70 \pi$ & 0,007 & *** \\
\hline armenia & 3737 & 2322 & 1,609 & 0,108 & & 5602 & 2028 & 2,762 & 0,006 & **** \\
\hline asean & 9848 & 2955 & 3,332 & 0,001 & $* * *$ & 10143 & 2507 & 4,045 & 0,000 & $* * *$ \\
\hline asiaeast & 20874 & 11954 & 1,746 & 0,081 & $*$ & 21382 & 11756 & 1,819 & 0,069 & 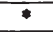 \\
\hline asiasoutheast & 23769 & 2696 & 8,816 & 0,000 & $* * *$ & 25692 & 2547 & 10,09 & 0,000 & *** \\
\hline assseasia & 14525 & 7500 & 1,937 & 0,053 & * & 16360 & 6623 & 2,470 & 0,014 & ** \\
\hline ausnzl & 4767 & 2379 & 2,003 & 0,045 & ** & 5702 & 2082 & 2,738 & 0,006 & $* * *$ \\
\hline ausnzlipn & 39558 & 3120 & 12,68 & 0,000 & $* * *$ & 40334 & 2699 & 14,94 & 0,000 & $* * *$ \\
\hline australia & 1781 & 2568 & 0,694 & 0,488 & & 5071 & 2099 & 2,417 & 0,016 & $* *$ \\
\hline austria & 7044 & 2439 & 2,888 & 0,004 & ****4 & 7693 & 1996 & 3,854 & 0,000 & *** \\
\hline azerbaijan & 3739 & 2322 & 1,610 & 0,107 & & 5602 & 2028 & 2,762 & 0,006 & $* * *$ \\
\hline bahamas & 3758 & 2322 & 1,618 & 0,106 & & 5617 & 2028 & 2,769 & 0,006 & $* * *$ \\
\hline bahrain & 3815 & 2442 & 1,562 & 0,118 & & 6033 & 2113 & 2,855 & 0.004 & $* * *$ \\
\hline bangladesh & 3402 & 2170 & 1,568 & 0,117 & & 3885 & 1965 & 1,977 & 0,048 & ** \\
\hline barbados & $377 \pi$ & 2367 & 1,596 & 0,111 & & 5780 & 2063 & 2,802 & 0,005 & **** \\
\hline belarus & -2401 & 4158 & $-0,578$ & 0,564 & & 72,32 & 3599 & 0,020 & 0,984 & \\
\hline belgium & 7497 & 2314 & 3,240 & 0,001 & *** & 7643 & 1951 & 3,917 & 0,000 & $* *$ \\
\hline belgiumlux & 3564 & 2995 & 1,190 & 0,234 & & 6876 & 2353 & 2,923 & 0,003 & $* * *$ \\
\hline belize & 3785 & 2367 & 1,599 & 0,110 & & 5785 & 2063 & 2,804 & 0,005 & $* * *$ \\
\hline
\end{tabular}




\begin{tabular}{|c|c|c|c|c|c|c|c|c|c|c|}
\hline Stichprobe $\rightarrow$ & & Ungewi & chtet mit & $\mathrm{B}+\mathrm{L}^{\mathrm{a}}$ & & & Igewich & et mit oh & ne $B+L^{a}$ & \\
\hline Dummy & Koeff. & $\begin{array}{r}\text { Std. } \\
\text { Fehl. }\end{array}$ & $t$-Wert & $\operatorname{Pr}(>|t|)$ & & Koeff. & $\begin{array}{r}\text { Std } \\
\text { Fehl. }\end{array}$ & $t$-Wert & $\operatorname{Pr}(>|t|)$ & \\
\hline benin & 3777 & 2367 & 1,596 & 0,111 & & 5783 & 2063 & 2,803 & 0,005 & **** \\
\hline bolivia & 3769 & 2367 & 1,592 & 0,111 & & 5775 & 2063 & 2,799 & 0,005 & **** \\
\hline botswana & 4509 & 2538 & 1,776 & 0,076 & * & 5778 & 2283 & 2,531 & 0,011 & $* *$ \\
\hline brazil & 4472 & 2441 & 1,833 & 0,067 & * & 5763 & 2071 & 2,783 & 0,005 & **** \\
\hline brunei & 3776 & 2367 & 1,595 & 0,111 & & 5782 & 2063 & 2,803 & 0,005 & **** \\
\hline bulgaria & 3605 & 2453 & 1,470 & 0,142 & & 5799 & 2105 & 2,755 & 0,006 & *** \\
\hline burkinafaso & 3776 & 2367 & 1,595 & 0,111 & & 5783 & 2063 & 2,803 & 0,005 & **** \\
\hline burundi & 3778 & 2367 & 1,596 & 0,110 & & 5786 & 2063 & 2,805 & 0,005 & **** \\
\hline c & -2992 & 4823 & $-0,620$ & 0,535 & & -2363 & 4799 & $-0,492$ & 0,622 & \\
\hline cairns & 4876 & 1365 & 3,571 & 0,000 & **** & $610 x$ & 1247 & 4,897 & 0,000 & **** \\
\hline cairnsdeveloped & $-893,6$ & 2897 & $-0,308$ & 0,758 & & 366,9 & 1808 & 0,203 & 0,839 & \\
\hline cairnsdeveloping & -4254 & 2426 & $-1,753$ & 0,080 & * & $-866,9$ & 1698 & $-0,511$ & 0,610 & \\
\hline cambodia & 3741 & 2322 & 1,611 & 0,107 & & 5607 & 2028 & 2,764 & 0,006 & **** \\
\hline cameroon & 1142 & 2951 & 0,387 & 0,699 & & 3328 & 2416 & 1,377 & 0,168 & \\
\hline canada & 1919 & 2453 & 0,782 & 0,434 & & 4650 & 2091 & 2,224 & 0,026 & ** \\
\hline capeverde & 3751 & 2322 & 1,615 & 0,106 & & 5617 & 2028 & 2,769 & 0,006 & **** \\
\hline carribean & 6268 & 2720 & 2,305 & 0,021 & ** & 7230 & 2501 & 2,891 & 0,004 & **** \\
\hline $\operatorname{ccs}$ & $-706,5$ & 13228 & $-0,053$ & 0,957 & & & & & & \\
\hline ceec & 8550 & 2617 & 3,268 & 0,001 & **** & 8504 & 2416 & 3,521 & 0,000 & **** \\
\hline centralafrrep & 3778 & 2367 & 1,596 & 0,110 & & 5785 & 2063 & 2,804 & 0,005 & **** \\
\hline centralamerica & 4658 & 2512 & 1,854 & 0,064 & * & 5887 & 2314 & 2,544 & 0,011 & ** \\
\hline cerntraleurope & -12890 & 4581 & $-2,814$ & 0,005 & **** & 7 & 7 & 7 & - & \\
\hline chad & 3780 & 2367 & 1,597 & 0,110 & & 5786 & 2063 & 2,805 & 0,005 & **** \\
\hline chile & -2189 & 3340 & $-0,655$ & 0,512 & & 5476 & 2356 & 2,325 & 0,020 & $* *$ \\
\hline china & 4061 & 2633 & 1,543 & 0,123 & & 6638 & 2176 & 3,051 & 0,002 & **** \\
\hline chinahk & -3813 & 4839 & $-0,788$ & 0,431 & & 2282 & 2705 & 0,844 & 0,399 & \\
\hline chinahks & 2404 & 2762 & 0,870 & 0,384 & & 4113 & 2463 & 1,670 & 0,095 & * \\
\hline colombia & 5052 & 2556 & 1,976 & 0,048 & ** & 6336 & 2275 & 2,785 & 0,005 & $* * *$ \\
\hline comoros & 3753 & 2322 & 1,616 & 0,106 & & 5618 & 2028 & 2,770 & 0,006 & **** \\
\hline congo & 3771 & 2367 & 1,593 & 0,111 & & 5778 & 2063 & 2,801 & 0,005 & **** \\
\hline congodemrep & 3894 & 2369 & 1,644 & 0,100 & & 5900 & 2064 & 2,859 & 0,004 & **** \\
\hline costarica & 3790 & 2367 & 1,601 & 0,109 & & 5793 & 2063 & 2,808 & 0,005 & **** \\
\hline cotedivore & 3755 & 2367 & 1,587 & 0,113 & & 5767 & 2062 & 2,796 & 0,005 & **** \\
\hline croatia & 2679 & 2462 & 1,088 & 0,276 & & 3721 & 2128 & 1,748 & 0,080 & * \\
\hline csamerica & -6931 & 5317 & $-1,304$ & 0,192 & & - & - & & & \\
\hline cuba & 3777 & 2367 & 1,596 & 0,111 & & 5783 & 2063 & 2,803 & 0,005 & $* * *$ \\
\hline cyprus & 3881 & 2367 & 1,640 & 0,101 & & 5888 & 2062 & 2,856 & 0,004 & **** \\
\hline cyprusmalta & 3698 & 2956 & 1,251 & 0,211 & & 6606 & 2362 & 2,797 & 0,005 & *** \\
\hline czech & 3675 & 2447 & 1,502 & 0,133 & & 5851 & 2106 & 2,779 & 0,005 & **** \\
\hline dcs & 83844 & 33894 & 2,474 & 0,013 & ** & 41490 & 13019 & 3,187 & 0,001 & **** \\
\hline denmark & 7299 & 2458 & 2,969 & 0,003 & *** & 7943 & 2003 & 3,965 & 0,000 & **** \\
\hline devasia & 18053 & 3412 & 5,291 & 0,000 & *** & 18005 & 3529 & 5,102 & 0,000 & **** \\
\hline developed & 13621 & 3014 & 4,519 & 0,000 & *** & 17332 & 2445 & 7,090 & 0,000 & **** \\
\hline developedasia & 2923 & 11216 & 0,261 & 0,794 & & $-64,30$ & 4786 & $-0,013$ & 0,989 & \\
\hline developing & 8334 & 3079 & 2,707 & 0,007 & *** & 10552 & 2786 & $3,78 \pi$ & 0,000 & $* * *$ \\
\hline djibouti & 3777 & 2367 & 1,596 & 0,111 & & 5783 & 2063 & 2,804 & 0,005 & **** \\
\hline dominica & 3782 & 2367 & 1,598 & 0,110 & & 5785 & 2062 & 2,806 & 0,005 & **** \\
\hline drit & -1007 & 5100 & $-0,198$ & 0,843 & & $-143,6$ & 5067 & $-0,028$ & 0,977 & \\
\hline eastasean & 16422 & 4228 & 3,884 & 0,000 & **** & 9530 & 2771 & 3,439 & 0,001 & **** \\
\hline eastasia & 4979 & 2415 & 2,062 & 0,039 & ** & 6130 & 2110 & 2,905 & 0,004 & **** \\
\hline easternasia & 5560 & 2271 & 2,448 & 0,014 & ** & 6626 & 2097 & 3,161 & 0,002 & **** \\
\hline eba & 6551 & 2256 & 2,904 & 0,004 & *** & 6401 & 1900 & 3,369 & 0,001 & **** \\
\hline ece & 1361 & 3452 & 0,394 & 0,693 & & 3405 & 2751 & 1,238 & 0,216 & \\
\hline ecuador & 3780 & 2367 & 1,597 & 0,110 & & 5787 & 2063 & 2,805 & 0,005 & **** \\
\hline
\end{tabular}




\begin{tabular}{|c|c|c|c|c|c|c|c|c|c|c|}
\hline \multirow{2}{*}{\begin{tabular}{|l|} 
Stichprobe $\rightarrow$ \\
Dummy
\end{tabular}} & \multicolumn{5}{|c|}{ Ungewichtet mit $B+L^{2}$} & \multicolumn{5}{|c|}{ Ungewichtet mit ohne $B+L^{2}$} \\
\hline & Koeff. & $\begin{array}{r}\text { Std } \\
\text { Fehl. }\end{array}$ & $\mathrm{t}$-Wert & $\operatorname{Pr}(>|t|)$ & & Koeff. & $\begin{array}{r}\text { Std } \\
\text { Fehl. }\end{array}$ & $\mathrm{t}$-Wert & $\operatorname{Pr}(>|t|)$ & \\
\hline ee & 4552 & 2326 & 1,957 & 0,050 & * & 5334 & 1996 & 2,673 & 0,008 & *** \\
\hline eec15 & 1748 & 2627 & 0,665 & 0,506 & & 4441 & 2133 & 2,082 & 0,037 & ** \\
\hline eeca & 3830 & 2401 & 1,595 & 0,111 & & 6219 & 2233 & 2,785 & 0,005 & *** \\
\hline eecas & 2356 & 1877 & 1,255 & 0,209 & & 3822 & 1721 & 2,221 & 0,026 & ** \\
\hline efta & 4425 & $240 \pi$ & 1,838 & 0,066 & + & 5560 & 2135 & 2,604 & 0,009 & **** \\
\hline egypt & 3752 & 2368 & 1,584 & 0,113 & & 5766 & 2062 & 2,797 & 0,005 & *** \\
\hline eit & 2527 & 2491 & 1,014 & 0,310 & & 4574 & 2116 & 2,162 & 0,031 & ** \\
\hline elsalvador & 3772 & 2367 & 1,594 & 0,111 & & 5773 & 2063 & 2,798 & 0,005 & **** \\
\hline emirates & 3809 & 2367 & 1,609 & 0,108 & & 5812 & 2063 & 2,818 & 0,005 & *** \\
\hline eritrea & 3749 & 2322 & 1,614 & 0,107 & & 5614 & 2028 & 2,768 & 0,006 & *** \\
\hline estonia & 3776 & 2442 & 1,546 & 0,122 & & 5930 & 2111 & 2,810 & 0,005 & **** \\
\hline ethiopia & 3735 & 2322 & 1,608 & 0,108 & & 5600 & 2028 & 2,761 & 0,006 & **** \\
\hline eu 10 & -1130 & 3176 & $-0,356$ & 0,722 & & 1504 & 2720 & 0,553 & 0,580 & \\
\hline eu 12 & 16896 & $517 \pi$ & 3,264 & 0,001 & **** & 21128 & 4887 & 4,323 & 0,000 & **** \\
\hline eul4 & -4583 & 6921 & $-0,662$ & 0,508 & & -1511 & 3292 & $-0,459$ & 0,646 & \\
\hline eu15 & 6402 & 3304 & 1,938 & 0,053 & * & 9264 & 2497 & 3,710 & 0,000 & *** \\
\hline eul 5 efta & 74659 & 33106 & 2,255 & 0,024 & $* *$ & 11568 & 3898 & 2,968 & 0,003 & *** \\
\hline eu25 & $1107 \pi$ & 4564 & $2,42 \pi$ & 0,015 & ** & 12378 & 4300 & 2,878 & 0,004 & **** \\
\hline eu25efta & 22278 & 4708 & 4,732 & 0,000 & $* * *$ & 24849 & 4141 & 6,001 & 0,000 & $* * *$ \\
\hline eu26 & -35583 & 14341 & $-2,481$ & 0,013 & $* *$ & -10897 & 7115 & $-1,531$ & 0,126 & \\
\hline eure & 10078 & 2732 & 3,688 & 0,000 & *** & 12265 & 2453 & 5,001 & 0,000 & *** \\
\hline eurest & 7411 & 4990 & 1,485 & 0,138 & & 8787 & 4814 & 1,825 & 0,068 & * \\
\hline europerest & 4214 & 2386 & 1,766 & 0,077 & $*$ & 6184 & 2124 & 2,912 & 0,004 & ***** \\
\hline fiji & 3752 & 2367 & 1,585 & 0,113 & & 5758 & 2064 & 2,790 & 0,005 & **** \\
\hline finland & 6946 & 2451 & 2,834 & 0,005 & $* * *$ & 7586 & 2000 & 3,793 & 0,000 & $* * *$ \\
\hline france & 5184 & 2483 & 2,088 & 0,037 & $* *$ & 6145 & 2108 & 2,915 & 0,004 & $* * *$ \\
\hline frenchpoly & 3749 & 2322 & 1,614 & 0,107 & & 5614 & 2028 & 2,768 & 0,006 & *** \\
\hline fsu & 4980 & 2528 & 1,970 & 0,049 & ** & 5297 & 2030 & 2,609 & 0,009 & **** \\
\hline gabon & 3776 & 2367 & 1,595 & 0,111 & & 5782 & 2063 & 2,803 & 0,005 & $* * *$ \\
\hline gambia & 3963 & 2529 & 1,567 & 0,117 & & 6000 & 2162 & 2,775 & 0,006 & **** \\
\hline georgia & 3733 & 2322 & 1,608 & 0,108 & & 5599 & 2028 & 2,760 & 0,006 & **** \\
\hline germany & 4114 & 2517 & 1,634 & 0,102 & & 5265 & 2075 & 2,537 & 0,011 & ** \\
\hline ghana & 3773 & 2367 & 1,594 & 0,111 & & 5780 & 2063 & 2,802 & 0,005 & **** \\
\hline greatbritain & 8074 & 2358 & 3,424 & 0,001 & *** & 8612 & 2034 & 4,235 & 0,000 & *** \\
\hline greece & 6812 & 2489 & 2,737 & 0,006 & *** & 7454 & 2015 & 3,699 & 0,000 & $* * *$ \\
\hline grenada & 3782 & 2367 & 1,598 & 0,110 & & 5785 & 2063 & 2,804 & 0,005 & $* * *$ \\
\hline grouptwenty & -6393 & 4200 & $-1,522$ & 0,128 & & $-663,8$ & 2217 & $-0,299$ & 0,765 & \\
\hline guatemala & 3791 & 2367 & 1,602 & 0,109 & & 5791 & 2063 & 2,807 & 0,005 & $* * *$ \\
\hline guinea & 3778 & 2367 & 1,596 & 0,110 & & 5785 & 2063 & 2,804 & 0,005 & $* * *$ \\
\hline guineabissau & 3779 & 2367 & 1,597 & 0,110 & & 5785 & 2063 & 2,804 & 0,005 & *** \\
\hline guyana & 3766 & 2367 & 1,591 & 0,112 & & 5773 & 2063 & 2,798 & 0,005 & $* * *$ \\
\hline haiti & 3775 & 2367 & 1,595 & 0,111 & & 5779 & 2063 & 2,801 & 0,005 & **** \\
\hline hiasia & 12611 & 2905 & 4,341 & 0,000 & **** & 13224 & 2564 & 5,159 & 0,000 & $* * *$ \\
\hline highincome & 143133 & 16841 & 8,499 & 0,000 & $* * *$ & 201215 & 22970 & 8,760 & 0,000 & $* * *$ \\
\hline hktaikorea & 14381 & 5383 & 2,672 & 0,008 & $* * *$ & 15621 & 4862 & 3,213 & 0,001 & **** \\
\hline hktaising & 7866 & 2640 & 2,980 & 0,003 & $* * *$ & 7810 & 2427 & 3,217 & 0,001 & $* * *$ \\
\hline honduras & 3773 & 2367 & 1,594 & 0,111 & & 5776 & 2063 & 2,800 & 0,005 & $* * *$ \\
\hline hongkong & -3347 & 3062 & $-1,093$ & 0,274 & & 2752 & 2184 & 1,260 & 0,208 & \\
\hline hongkongsing & 5055 & 2336 & 2,164 & 0,031 & $* *$ & 6354 & 2139 & 2,970 & 0,003 & $* * *$ \\
\hline hungary & 3651 & 2448 & 1,491 & 0,136 & & 5825 & 2105 & 2,767 & 0,006 & $* * *$ \\
\hline iceland & 3836 & 2367 & 1,620 & 0,105 & & 5838 & 2062 & 2,831 & 0,005 & $* * *$ \\
\hline india & 1428 & 2580 & 0,554 & 0,580 & & 3927 & 2111 & 1,861 & 0,063 & * \\
\hline indonesia & 1425 & 2648 & 0,538 & 0,590 & & 6104 & 2158 & 2,829 & 0,005 & $* * *$ \\
\hline iran & 3573 & 2370 & 1,508 & 0,132 & & 5603 & 2062 & 2,718 & $0,00 \pi$ & **** \\
\hline
\end{tabular}




\begin{tabular}{|c|c|c|c|c|c|c|c|c|c|c|}
\hline Stichprobe $\rightarrow$ & & Ungewi & htet mit & $\mathrm{B}+\mathrm{L}^{\mathrm{a}}$ & & & ngewich & et mit oh & $\mathrm{B} B+L^{\mathrm{a}}$ & \\
\hline Dummy & Koeff. & $\begin{array}{r}\text { Std } \\
\text { Fehl. }\end{array}$ & $t$-Wert & $\operatorname{Pr}(>|t|)$ & & Koeff. & $\begin{array}{r}\text { Std. } \\
\text { Fehl. }\end{array}$ & $t$-Wert & $\operatorname{Pr}(>|t|)$ & \\
\hline iraq & 3686 & 2322 & 1,587 & 0,113 & & 5545 & 2028 & 2,734 & 0,006 & **** \\
\hline ireland & 6769 & 2282 & 2,966 & 0,003 & **** & 7061 & 1934 & 3,651 & 0,000 & $* * *$ \\
\hline israel & 4076 & 2369 & 1,721 & 0,085 & * & 6079 & 2061 & 2,949 & 0,003 & **** \\
\hline italy & 6375 & 2538 & 2,512 & 0,012 & ** & 7401 & 2046 & 3,617 & 0,000 & **** \\
\hline jamaica & 3748 & $236 \pi$ & 1,584 & 0,113 & & 5754 & 2063 & 2,789 & 0,005 & $* * *$ \\
\hline japan & 11668 & 3968 & 2,941 & 0,003 & $* * *$ & 10813 & 2176 & 4,970 & 0,000 & $* * *$ \\
\hline japankorea & 14976 & 5248 & 2,853 & 0,004 & **** & 15570 & 5058 & 3,078 & 0,002 & **** \\
\hline jordan & 4380 & 2487 & 1,761 & 0,078 & * & 3580 & 2553 & 1,402 & 0,161 & \\
\hline kazakhstan & -5001 & 4217 & $-1,186$ & 0,236 & & -2272 & 3558 & $-0,638$ & 0,523 & \\
\hline kenya & 3744 & 2322 & 1,612 & 0,107 & & 5611 & 2028 & 2,766 & 0,006 & **** \\
\hline korea & 4070 & 3032 & 1,342 & 0,180 & & 8796 & 2261 & 3,891 & 0,000 & **** \\
\hline koreadpr & 3735 & 2322 & 1,608 & 0,108 & & 5600 & 2028 & 2,761 & 0,006 & *** \\
\hline koreataiwan & 12628 & 2932 & 4,307 & 0,000 & **** & 14023 & 2661 & 5,270 & 0,000 & **** \\
\hline kuwait & 3819 & 2367 & 1,614 & 0,107 & & 5822 & 2062 & 2,823 & 0,005 & **** \\
\hline kyrgyzstan & 3783 & 2367 & 1,598 & 0,110 & & 5789 & 2063 & 2,806 & 0,005 & **** \\
\hline laos & 3754 & 2322 & 1,616 & 0,106 & & 5619 & 2028 & 2,771 & 0,006 & **** \\
\hline latin & 6548 & 2570 & 2,548 & 0,011 & ** & 8055 & 2252 & 3,577 & 0,000 & *** \\
\hline latvia & 3758 & 2442 & 1,539 & 0,124 & & 5914 & 2110 & 2,803 & 0,005 & **** \\
\hline leastdeveloped & 13241 & 5119 & 2,587 & 0,010 & $* * *$ & 8982 & 2774 & 3,238 & 0,001 & **** \\
\hline lebanon & 3768 & 2322 & 1,623 & 0,105 & & 5629 & 2028 & 2,775 & 0,006 & ***** \\
\hline lesotho & 3767 & 2322 & 1,622 & 0,105 & & 5632 & 2028 & 2,777 & 0,006 & **** \\
\hline liaexp & -1369 & 2610 & $-0,525$ & 0,600 & & 1964 & 2204 & 0,891 & 0,373 & \\
\hline liberia & 3750 & 2322 & 1,615 & 0,106 & & 5616 & 2028 & 2,769 & 0,006 & *** \\
\hline libya & 3734 & 2322 & 1,608 & 0,108 & & 5600 & 2028 & 2,761 & 0,006 & *** \\
\hline liexp & -2023 & 3312 & $-0,611$ & 0,541 & & 2058 & 2549 & $0,80 \pi$ & 0,420 & \\
\hline $\operatorname{limp}$ & 1097 & 2664 & 0,412 & 0,681 & & 4362 & 2306 & 1,892 & 0,059 & * \\
\hline lithuania & $373 x$ & 2442 & 1,530 & 0,126 & & 5895 & 2108 & 2,796 & 0,005 & **** \\
\hline Imiimp & $-978,7$ & 2702 & $-0,362$ & 0,717 & & 2664 & 2222 & 1,199 & 0,231 & \\
\hline lowincom & 4084 & 8172 & 0,500 & 0,617 & & 19610 & 3130 & 6,265 & 0,000 & **** \\
\hline macao & 3777 & 2367 & 1,596 & 0,111 & & 5784 & 2063 & 2,804 & 0,005 & **** \\
\hline macedonia & 3749 & 2322 & 1,615 & 0,106 & & 5615 & 2028 & 2,769 & 0,006 & $* * *$ \\
\hline madagascar & 4699 & 2558 & 1,837 & 0,066 & * & 6578 & 2265 & 2,904 & 0,004 & $* * *$ \\
\hline maghreb & 11070 & 2652 & 4,174 & 0,000 & **** & 11031 & 2443 & 4,516 & 0,000 & **** \\
\hline malawi & 4463 & 2455 & 1,818 & 0,069 & $*$ & 6181 & 2170 & 2,849 & 0,004 & *** \\
\hline malaysia & -2389 & 3156 & $-0,757$ & 0,449 & & 5324 & 2195 & 2,426 & 0,015 & ** \\
\hline maledives & 3777 & 2367 & 1,596 & 0,111 & & 5783 & 2063 & 2,803 & 0,005 & **** \\
\hline mali & 3775 & 2367 & 1,595 & 0,111 & & 5783 & 2063 & 2,804 & 0,005 & $* * *$ \\
\hline malta & 3746 & 2322 & 1,613 & 0,107 & & 5612 & 2028 & 2,767 & 0,006 & *** \\
\hline marrocco & 170,8 & 3042 & 0,056 & 0,955 & & 6402 & 2296 & 2,788 & 0,005 & **** \\
\hline mauritania & 3774 & 2367 & 1,595 & 0,111 & & 5780 & 2063 & 2,802 & 0,005 & **** \\
\hline mauritius & 3730 & 2367 & 1,576 & 0,115 & & 5739 & 2064 & 2,781 & 0,005 & *** \\
\hline mediterranean & 5668 & 3074 & 1,844 & 0,065 & * & 6466 & 2624 & 2,465 & 0,014 & *** \\
\hline mena & 5855 & 2673 & 2,191 & 0,029 & *** & 7043 & 2428 & 2,901 & 0,004 & **** \\
\hline mer & 912,6 & 3482 & 0,262 & 0,793 & & 3122 & 2750 & 1,135 & 0,256 & \\
\hline mercosur & 6554 & 2325 & 2,819 & 0,005 & **** & 7676 & 2099 & 3,657 & 0,000 & *** \\
\hline mexico & 1066 & 2583 & 0,413 & 0,680 & & 4741 & 2136 & 2,219 & 0,026 & *** \\
\hline miaexp & -1782 & 2819 & $-0,632$ & 0,527 & & 2272 & 2232 & 1,018 & 0,309 & \\
\hline middleeast & 5946 & 2327 & 2,555 & 0,011 & ** & 7269 & 2176 & 3,340 & 0,001 & *** \\
\hline middleincome & 124887 & 14436 & 8,651 & 0,000 & **** & 163306 & 10630 & 15,36 & 0,000 & **** \\
\hline $\operatorname{miexp}$ & 3256 & 3009 & 1,082 & 0,279 & & 7251 & 2821 & 2,571 & 0,010 & $* *$ \\
\hline mifexp & 5629 & 4029 & 1,397 & 0,162 & & 9244 & 3984 & 2,320 & 0,020 & $* *$ \\
\hline mna & 2590 & 2476 & 1,046 & 0,296 & & 4471 & 2108 & 2,121 & 0,034 & ** \\
\hline moldova & 3756 & 2322 & 1,617 & 0,106 & & 5621 & 2028 & 2,772 & 0,006 & $* * *$ \\
\hline mongolia & 3818 & 2442 & 1,563 & 0,118 & & 6036 & 2113 & $2,85 x$ & 0,004 & **** \\
\hline
\end{tabular}




\begin{tabular}{|c|c|c|c|c|c|c|c|c|c|c|}
\hline Stichprobe $\rightarrow$ & & Ungewi & htet mit & $B+L^{2}$ & & & gewich & et mit oh & $\mathrm{ne} B+L^{2}$ & \\
\hline Dummy & Koeff. & $\begin{array}{r}\text { Std } \\
\text { Fehl }\end{array}$ & $t$-Wert & $\operatorname{Pr}(>|t|)$ & & Koeff. & $\begin{array}{r}\text { Std. } \\
\text { Fehl. }\end{array}$ & t-Wert & $\operatorname{Pr}(>|t|)$ & \\
\hline mozambique & 5258 & 2379 & 2,210 & $0,02 \pi$ & ** & 6388 & 2129 & 3,001 & 0,003 & *** \\
\hline myanmar & 3818 & 2442 & 1,563 & 0,118 & & 6036 & 2113 & 2,857 & 0,004 & $* * *$ \\
\hline nafta & 1955 & 5093 & 0,384 & 0,701 & & 5158 & 4317 & 1,195 & 0,232 & \\
\hline namibia & 3763 & 2322 & 1,620 & 0,105 & & 5628 & 2028 & 2,775 & 0,006 & $* * *$ \\
\hline nea & 9546 & 2961 & 3,223 & 0,001 & $* * *$ & 11039 & 2809 & 3,930 & 0,000 & $* * *$ \\
\hline nepal & 3748 & 2322 & 1,614 & 0,107 & & 5613 & 2028 & 2,768 & 0,006 & $* * *$ \\
\hline nethant & 3749 & 2322 & 1,615 & 0,106 & & 5615 & 2028 & 2,768 & 0,006 & $* * *$ \\
\hline netherland & 3991 & 2377 & 1,679 & 0,093 & $*$ & 4773 & 2030 & 2,351 & 0,019 & $*$ \\
\hline nic & -2679 & 3555 & $-0,754$ & 0,451 & & 540 & 2426 & 0,222 & 0,824 & \\
\hline nicaragua & 3786 & 2367 & 1,599 & 0,110 & & 5787 & 2063 & 2,805 & 0,005 & $* * *$ \\
\hline nicasia & 1828 & 3344 & 0,547 & 0,585 & & 3850 & 2832 & 1,359 & 0,174 & \\
\hline niger & 3776 & 2367 & 1,595 & 0,111 & & 5782 & 2063 & 2,803 & 0,005 & *** \\
\hline nigeria & 4607 & 2456 & 1,876 & 0,061 & $*$ & 6303 & 2081 & 3,029 & 0,002 & $* * *$ \\
\hline nis & 3177 & 3394 & 0,936 & 0,349 & & 4666 & 2761 & 1,690 & 0,091 & * \\
\hline $\mathrm{nmb}$ & -1472 & 4204 & $-0,350$ & 0,726 & & 5122 & 2775 & 1,846 & 0,065 & $*$ \\
\hline northafrica & 6191 & 2580 & 2,399 & 0,016 & ** & 4580 & 2181 & 2,099 & 0,036 & $*$ \\
\hline northamerica & 9892 & 3190 & 3,101 & 0,002 & $* * *$ & 9281 & 2999 & 3,095 & 0,002 & $* * *$ \\
\hline northeastasia & 21501 & 3796 & 5,664 & 0,000 & $* *$ & 22860 & 3725 & 6,137 & 0,000 & $* * *$ \\
\hline norway & 4205 & 2372 & 1,773 & 0,076 & $*$ & 6206 & 2063 & 3,009 & 0,003 & $* * *$ \\
\hline $\mathrm{nzl}$ & $-235,7$ & 2766 & $-0,085$ & 0,932 & & 4800 & 2192 & 2,190 & 0,029 & $*$ \\
\hline oasia & 4821 & 2721 & 1,772 & 0,076 & * & 7135 & 2412 & 2,959 & 0,003 & $* * *$ \\
\hline oasp & 11505 & 3150 & 3,653 & 0,000 & $* * *$ & 13843 & 2778 & 4,984 & 0,000 & $* * *$ \\
\hline oaspa & 2025 & 6142 & 0,330 & 0,742 & & 1782 & $574 \pi$ & 0,310 & $0,75 \pi$ & \\
\hline oaspac & 6035 & 2495 & 2,419 & 0,016 & ** & 6682 & $223 x$ & 2,987 & 0,003 & $* * *$ \\
\hline ocd & -1829 & 3742 & $-0,489$ & 0,625 & & 1319 & 2762 & 0,477 & 0,633 & \\
\hline oceania & 6102 & 2438 & 2,503 & 0,012 & ** & 7297 & 2301 & 3,171 & 0,002 & $* *$ \\
\hline oeasia & 6954 & 2507 & 2,773 & 0,006 & $* * *$ & 6721 & 2268 & 2,963 & 0,003 & $* * *$ \\
\hline oeefsu & 4101 & 2326 & 1,763 & 0,078 & $*$ & 5809 & 2129 & 2,728 & 0,006 & $* *$ \\
\hline oeurope & 7377 & 2773 & 2,660 & 0,008 & $* * *$ & 10138 & 2314 & 4,381 & 0,000 & $* * *$ \\
\hline olac & 3243 & 2563 & 1,265 & 0,206 & & 4959 & 2292 & 2,164 & 0,031 & ** \\
\hline olat & 5349 & 2378 & 2,249 & 0,025 & ** & 6007 & 2143 & 2,804 & 0,005 & $* * *$ \\
\hline oman & 3815 & 2442 & 1,562 & 0,118 & & 6033 & 2113 & 2,855 & 0,004 & $* * *$ \\
\hline onam & 6519 & 2605 & 2,503 & 0,012 & ** & 6479 & 2345 & 2,762 & 0,006 & $* * *$ \\
\hline osea & 5187 & 2608 & 1,989 & 0,047 & $* *$ & 6762 & 2317 & 2,918 & 0,004 & $* * *$ \\
\hline othapec & 5443 & 2877 & 1,892 & 0,059 & $*$ & 6074 & 2079 & 2,921 & 0,004 & $* * *$ \\
\hline othasia & 6638 & 2556 & 2,597 & 0,009 & $* * *$ & 7445 & $233 \pi$ & 3,185 & 0,001 & $* 4 *$ \\
\hline othdeveloped & 5128 & 2675 & 1,917 & 0,055 & * & 6123 & 2410 & 2,541 & 0,011 & ** \\
\hline otheca & 5803 & 2382 & 2,436 & 0,015 & ** & 6940 & 1980 & 3,505 & 0,000 & $* * *$ \\
\hline otherafrica & 5786 & 2382 & 2,429 & 0,015 & $* *$ & 6923 & 1980 & 3,497 & 0,000 & $* * *$ \\
\hline othhiasia & 4550 & 3610 & 1,261 & 0,208 & & 4951 & 2526 & 1,960 & 0,050 & $*$ \\
\hline othlatin & 5613 & 3195 & $1,75 \pi$ & 0,079 & * & 6812 & 2759 & 2,469 & 0,014 & ** \\
\hline othnic & $604 \pi$ & 2746 & 2,203 & 0,028 & $* *$ & 7545 & 2307 & 3,271 & 0,001 & $* * *$ \\
\hline othsea & 5691 & 2164 & 2,630 & 0,009 & *** & 7182 & 1888 & 3,804 & 0,000 & $* * *$ \\
\hline \begin{tabular}{|l} 
othseasia \\
\end{tabular} & 1614 & 3110 & 0,519 & 0,604 & & 3679 & 2646 & 1,390 & 0,164 & \\
\hline othsouthasia & -3750 & 3643 & $-1,029$ & 0,303 & & 4039 & 2716 & $1,48 \pi$ & 0,137 & \\
\hline owe & 3748 & 2324 & 1,613 & 0,107 & & 5590 & 2143 & 2,608 & 0,009 & $* * *$ \\
\hline pakistan & 3670 & 2370 & 1,549 & 0,121 & & 5696 & 2061 & 2,763 & 0,006 & $* * *$ \\
\hline panama & 3784 & 2367 & 1,599 & 0,110 & & 5784 & 2063 & 2,804 & 0,005 & $* * *$ \\
\hline papua & 3782 & $236 x$ & 1,598 & 0,110 & & 5789 & 2063 & 2,806 & 0,005 & $* * *$ \\
\hline paraguay & 3780 & 2367 & 1,597 & 0,110 & & 5786 & 2063 & 2,805 & 0,005 & $\$ 4$ \\
\hline peru & 5267 & 2546 & 2,069 & 0,039 & ** & 6176 & 2315 & 2,668 & 0,008 & *** \\
\hline philippines & -1622 & 2942 & $-0,551$ & 0,581 & & 4438 & 2217 & 2,002 & 0,045 & $* *$ \\
\hline poland & 3172 & 2508 & 1,265 & 0,206 & & 5435 & 2124 & 2,558 & 0,011 & $*$ \\
\hline portugal & 6763 & 2456 & 2,754 & 0,006 & **** & 7410 & 2001 & 3,703 & 0,000 & $* * *$ \\
\hline
\end{tabular}




\begin{tabular}{|c|c|c|c|c|c|c|c|c|c|c|}
\hline Stichprobe - & & Ungewi & chtet mit & $\mathrm{B}+\mathrm{L}^{\mathrm{a}}$ & & & ngewicht & et mit oh & $\mathrm{Be}+\mathrm{L}^{\mathrm{B}}$ & \\
\hline Dummy & Koeff. & $\begin{array}{r}\text { Std. } \\
\text { Fehl. }\end{array}$ & $\mathrm{t}$-Wert & $\operatorname{Pr}(>|t|)$ & & Koeff. & $\begin{array}{r}\text { Std. } \\
\text { Fehl. }\end{array}$ & $t$-Wert & $\operatorname{Pr}(>|t|) \mid$ & \\
\hline qatar & 3818 & 2442 & 1,563 & 0,118 & & 6036 & 2113 & $2,85 x$ & 0,004 & $* * *$ \\
\hline racp & 6728 & 2258 & 2,980 & 0,003 & **** & 6578 & 1902 & 3,458 & 0,001 & **** \\
\hline rafrica & 4939 & 2254 & 2,191 & 0,028 & *** & 5939 & 2067 & 2,873 & 0,004 & **** \\
\hline rasia & -1262 & 4025 & $-0,314$ & 0,754 & & 7454 & 2111 & 3,530 & 0,000 & **** \\
\hline rcaims & 3049 & 2916 & 1,046 & 0,296 & & 5256 & 2546 & 2,064 & 0,039 & $* *$ \\
\hline rcarribbean & 9110 & 1859 & 4,902 & 0,000 & **** & 10690 & 1743 & 6,134 & 0,000 & **** \\
\hline rdeveloped & 3272 & 2217 & 1,476 & 0,140 & & 4606 & 2077 & 2,217 & 0,027 & *** \\
\hline rea & 2508 & 2605 & 0,963 & 0,336 & & 3975 & 2251 & 1,766 & 0,078 & * \\
\hline reastasia & 23323 & 11656 & 2,001 & 0,045 & ** & 24027 & 11561 & 2,078 & 0,038 & ** \\
\hline reca & 1910 & 2591 & 0,737 & 0,461 & & 2782 & 2127 & 1,308 & 0,191 & \\
\hline refta & 6499 & 2244 & 2,896 & 0,004 & **** & 6443 & 1900 & 3,391 & 0,001 & **** \\
\hline restasia & 471,4 & 1821 & 0,259 & 0,796 & & 2051 & 1702 & 1,205 & 0,228 & \\
\hline reu & 2542 & 3385 & 0,751 & 0,453 & & 5253 & $244 \pi$ & $2,14 x$ & 0,032 & ** \\
\hline reurope & -7749 & $362 \pi$ & $-2,136$ & 0,033 & ** & -6401 & 2410 & $-2,656$ & 0,008 & **** \\
\hline rlatin & 4932 & 2584 & 1,909 & 0,056 & * & 5595 & 2209 & 2,532 & 0,011 & $* *$ \\
\hline rme & -3576 & 10307 & $-0,347$ & 0,729 & & -1 & 7 & 7 & I & \\
\hline rmiddlefast & 5840 & $273 \pi$ & 2,134 & 0,033 & ** & 7013 & 2554 & 2,746 & 0,006 & **** \\
\hline morthafrica & 4479 & 2831 & 1,582 & 0,114 & & 5878 & 2526 & 2,327 & 0,020 & *** \\
\hline roasia & 5654 & 2410 & 2,346 & 0,019 & ** & 7531 & 2255 & 3,340 & 0,001 & $* * *$ \\
\hline roecd & 6667 & 2484 & 2,685 & 0,007 & $* * *$ & 7562 & 2393 & 3,160 & 0,002 & *** \\
\hline romania & 4159 & 2451 & 1,697 & 0,090 & * & 6362 & 2117 & 3,006 & 0,003 & **** \\
\hline row & 31308 & 13559 & 2,309 & 0,021 & ** & 12593 & 5355 & 2,352 & 0,019 & ** \\
\hline rsa & -1345 & 2266 & $-0,593$ & 0,553 & & $-190,0$ & 2046 & $-0,093$ & 0,926 & \\
\hline rsacu & 3451 & 2983 & 1,157 & $0,24 \pi$ & & 6690 & 2353 & 2,843 & 0,004 & **** \\
\hline rsothasia & 5191 & 2405 & 2,158 & 0,031 & ** & 6306 & 2174 & 2,900 & 0,004 & **** \\
\hline rsouthafrica & 3586 & 2884 & 1,243 & 0,214 & & 6032 & 2387 & 2,527 & 0,012 & ** \\
\hline rsouthamerica & 6963 & 2647 & 2,630 & 0,009 & *** & 6630 & $245 \pi$ & 2,698 & $0,00 x$ & **** \\
\hline rsouthernafrica & 6015 & 2584 & 2,328 & 0,020 & ** & 6385 & 2379 & 2,684 & 0,007 & **** \\
\hline rssa & 3460 & 2325 & 1,488 & 0,137 & & 5326 & 2032 & 2,621 & 0,009 & $* * *$ \\
\hline rsubsaharan & 3656 & 2405 & 1,520 & 0,129 & & 4890 & 2102 & $2,32 \pi$ & 0,020 & ** \\
\hline russia & 2817 & 2403 & 1,172 & 0,241 & & 4167 & 2164 & 1,926 & 0,054 & * \\
\hline rwanda & 3779 & 2367 & 1,597 & 0,110 & & 5785 & 2063 & 2,804 & 0,005 & **** \\
\hline saarc & 4032 & 2319 & 1,739 & 0,082 & * & 5983 & 2093 & 2,858 & 0,004 & **** \\
\hline sacu & 2033 & 2679 & 0,759 & 0,448 & & 5959 & 2222 & 2,681 & 0,007 & *** \\
\hline saf & 4975 & 2285 & 2,178 & 0,029 & ** & 5147 & 2040 & 2,524 & 0,012 & ** \\
\hline salomon & 3780 & 2367 & 1,597 & 0,110 & & 5786 & 2063 & 2,805 & 0,005 & **** \\
\hline saotome & 3753 & 2322 & 1,616 & 0,106 & & 5619 & 2028 & 2,770 & 0,006 & $* * *$ \\
\hline saudiarabia & 3696 & 2367 & 1,561 & 0,118 & & 5699 & 2065 & 2,760 & 0,006 & $* * *$ \\
\hline sea & 8321 & 2336 & 3,562 & 0,000 & **** & 9423 & 2180 & 4,322 & 0,000 & **** \\
\hline selectedssa & 2176 & 2437 & 0,893 & 0,372 & & 3313 & 2214 & 1,496 & 0,135 & \\
\hline senegal & 3982 & 2529 & 1,574 & 0,116 & & 6019 & 2162 & 2,784 & 0,005 & $* * *$ \\
\hline seychelles & 3753 & 2322 & 1,616 & 0,106 & & 5618 & 2028 & 2,770 & 0,006 & **** \\
\hline sierraleone & 3778 & 2367 & 1,596 & 0,110 & & 5785 & 2063 & 2,804 & 0,005 & *** \\
\hline singapore & -4240 & 3270 & $-1,297$ & 0,195 & & 3485 & 2334 & 1,493 & 0,136 & \\
\hline slovakia & 3734 & 2443 & 1,528 & 0,126 & & 5894 & 2109 & 2,795 & 0,005 & **** \\
\hline slovenia & 3731 & 2443 & 1,527 & 0,127 & & 5894 & 2109 & 2,795 & 0,005 & **** \\
\hline somalia & 3753 & 2322 & 1,616 & 0,106 & & 5618 & 2028 & 2,770 & 0,006 & **** \\
\hline southamerica & 6713 & 3112 & 2,157 & 0,031 & ** & 6189 & 2290 & 2,703 & 0,007 & **** \\
\hline southasia & 8596 & 2983 & 2,881 & 0,004 & **** & 9748 & 2720 & 3,584 & 0,000 & $* * *$ \\
\hline southernafr & 5292 & 2400 & 2,205 & 0,027 & *** & 6085 & 2278 & 2,672 & 0,008 & *** \\
\hline southerncone & 3977 & 3606 & 1,103 & 0,270 & & 4378 & 2524 & 1,735 & 0,083 & * \\
\hline spain & 7334 & 2588 & 2,834 & 0,005 & **** & 8076 & 2049 & 3,941 & 0,000 & **** \\
\hline srilanka & -1015 & 3306 & $-0,307$ & 0,759 & & $624 \pi$ & 2303 & 2,713 & 0,007 & **** \\
\hline ssa & 3868 & 2263 & 1,709 & 0,087 & * & 4190 & 2025 & 2,069 & 0,039 & ** \\
\hline
\end{tabular}




\begin{tabular}{|c|c|c|c|c|c|c|c|c|c|c|}
\hline \multirow{2}{*}{\begin{tabular}{|l|} 
Stichprobe $\rightarrow$ \\
Dummy
\end{tabular}} & \multicolumn{5}{|c|}{ Ungewichtet mit $\mathrm{B}+\mathrm{L}^{\mathbf{a}}$} & \multicolumn{5}{|c|}{ Ungewichtet mit ohne $\mathrm{B}+\mathrm{L}^{\mathrm{a}}$} \\
\hline & Koeff. & $\begin{array}{r}\text { Std. } \\
\text { Fehl. }\end{array}$ & t-Wert & $\operatorname{Pr}(>|t|)$ & & Koeff. & $\begin{array}{r}\text { Std } \\
\text { Fehl. }\end{array}$ & t-Wert & $\operatorname{Pr}(>|t|)$ & \\
\hline ssasacu & 1855 & 2504 & 0,741 & 0,459 & & 4627 & 2279 & 2,031 & 0,042 & *** \\
\hline stkitts & 3829 & 2442 & 1,568 & 0,117 & & 6046 & 2113 & 2,862 & 0,004 & $* *$ \\
\hline stlucia & 3791 & 2367 & 1,602 & 0,109 & & 5793 & 2063 & 2,808 & 0,005 & *** \\
\hline stvincent & 3788 & 2367 & 1,600 & 0,110 & & 5790 & 2063 & 2,807 & 0,005 & ***** \\
\hline sudan & 3769 & 2367 & 1,592 & 0,111 & & 5776 & 2063 & 2,800 & 0,005 & $* * *$ \\
\hline suriname & 3780 & 2367 & 1,597 & 0,110 & & 5787 & 2063 & 2,805 & 0,005 & $* * *$ \\
\hline swaziland & 3764 & 2322 & 1,621 & 0,105 & & 5630 & 2028 & 2,776 & 0,006 & $* * *$ \\
\hline sweden & 2850 & 2832 & 1,006 & 0,314 & & 4144 & 2219 & 1,867 & 0,062 & + \\
\hline switzerland & 4128 & 2667 & 1,548 & 0,122 & & 5257 & 2142 & 2,455 & 0,014 & ** \\
\hline syria & 3754 & 2322 & 1,616 & 0,106 & & 5612 & 2028 & 2,767 & 0,006 & $* *$ \\
\hline$t$ & 14225 & 7613 & 1,869 & 0,062 & - & 14398 & 7146 & 2,015 & 0,044 & ** \\
\hline taiwan & 1376 & 2850 & 0,483 & 0,629 & & 4822 & 2124 & 2,271 & 0,023 & ** \\
\hline tajkistan & 3744 & 2322 & 1,612 & 0,107 & & 5610 & 2028 & 2,766 & 0,006 & $\% * *$ \\
\hline tanzania & 4654 & 2389 & 1,948 & 0,051 & $*$ & 6081 & 2166 & 2,807 & 0,005 & *** \\
\hline thaijapkorea & -11622 & 4431 & $-2,623$ & 0,009 & *\% & -8235 & 3397 & $-2,424$ & 0,015 & ** \\
\hline thailand & 363,1 & 2791 & 0,130 & 0,896 & & 5873 & 2157 & 2,722 & 0,007 & *** \\
\hline tigers & 7331 & 2705 & 2,711 & 0,007 & $* * *$ & 7156 & 2489 & 2,875 & 0,004 & *** \\
\hline togo & 3776 & 2367 & 1,595 & 0,111 & & 5783 & 2063 & 2,803 & 0,005 & $* * *$ \\
\hline trans & 4507 & 2457 & 1,835 & 0,067 & * & 5545 & 2322 & 2,388 & 0,017 & ** \\
\hline trinidad & 3778 & 2367 & 1,596 & 0,110 & & 5781 & 2063 & 2,802 & 0,005 & *** \\
\hline tuc & 351,2 & 3086 & 0,114 & 0,909 & & 954,5 & 2968 & 0,322 & 0,748 & \\
\hline tunesia & 4802 & 2574 & 1,865 & 0,062 & + & 6699 & 2284 & 2,933 & 0,003 & $* * *$ \\
\hline turkey & 2611 & 2520 & 1,036 & 0,300 & & 5461 & 2128 & 2,567 & 0,010 & ** \\
\hline turkmenistan & 3750 & 2322 & 1,615 & 0,106 & & 5614 & 2028 & 2,768 & 0,006 & $* * *$ \\
\hline uganda & 4946 & 2340 & 2,113 & 0,035 & $*$ & 6308 & 2085 & 3,025 & 0,002 & $* *$ \\
\hline ukraine & 3841 & 2323 & 1,654 & 0,098 & - & 5708 & 2028 & 2,814 & 0,005 & *** \\
\hline umiimp & 1317 & 2750 & 0,479 & 0,632 & & 4971 & 2394 & 2,077 & 0,038 & $*$ \\
\hline uruguay & 4898 & 2733 & 1,793 & 0,073 & $*$ & 6021 & 2397 & 2,512 & 0,012 & ** \\
\hline usa & 15785 & 4710 & 3,352 & 0,001 & *** & 7546 & 2063 & $3,65 x$ & 0,000 & **** \\
\hline usbekistan & 3737 & 2322 & 1,609 & 0,108 & & 5602 & 2028 & 2,762 & 0,006 & *** \\
\hline ussr & 4257 & 2434 & 1,749 & 0,080 & + & 6119 & 2074 & 2,951 & 0,003 & *** \\
\hline vanuatu & 3753 & 2322 & 1,616 & 0,106 & & 5619 & 2028 & 2,770 & 0,006 & $* * *$ \\
\hline venezuela & 5092 & 2542 & 2,004 & 0,045 & ** & 6009 & 2309 & 2,603 & 0,009 & $* * *$ \\
\hline vietnam & 3671 & 2408 & 1,525 & 0,127 & & 4858 & 2174 & 2,235 & 0,025 & ** \\
\hline westerneurope & 18522 & 4140 & 4,474 & 0,000 & $* * *$ & 21056 & 3637 & 5,790 & 0,000 & $* * *$ \\
\hline yemen & 3690 & 2322 & 1,589 & 0,112 & & 5553 & 2028 & 2,737 & 0,006 & $4 * 4$ \\
\hline yugoslavia & 3765 & 2323 & 1,621 & 0,105 & & 5633 & 2028 & 2,777 & 0,005 & *** \\
\hline zambia & 4778 & 2331 & 2,050 & 0,040 & $*$ & 6141 & 2067 & 2,971 & 0,003 & *** \\
\hline zimbabwe & 4251 & 2560 & 1,661 & 0,097 & * & 5970 & 2273 & 2,626 & 0,009 & $* * *$ \\
\hline
\end{tabular}

Quelle: Eigene Berechnung. 
Tabelle 15: Koeffizienten regionaler Aggregationen (gewichtet).

\begin{tabular}{|c|c|c|c|c|c|c|c|c|c|c|}
\hline \multirow{3}{*}{$\begin{aligned} \text { Gewichtung } \rightarrow \\
\text { Stichprobe } \rightarrow \\
\text { Dummy }\end{aligned}$} & \multicolumn{10}{|c|}{ Gewichtet mit [1/(Beobachtungen in der Studie)] } \\
\hline & \multicolumn{5}{|c|}{ mit $B+L^{\circ}$} & \multicolumn{5}{|c|}{ ohne B+L" } \\
\hline & Koeff. & Std. Fehl. & $t$-Wert & $\operatorname{Pr}(>|t|)$ & & Koeff. & Std. Fehl. & $t$-Wert & $\operatorname{Pr}(>|t|)$ & \\
\hline afghanistan & 9065 & 913,2 & 9,926 & 0,000 & **** & 9431 & 1645 & 5,732 & 0,000 & **** \\
\hline africa & 5657 & 1126 & 5,026 & 0,000 & *** & 5731 & 1866 & 3,072 & 0,002 & **** \\
\hline albania & 9542 & 1083 & 8,811 & 0,000 & **** & 10184 & 3078 & 3,308 & 0,001 & **** \\
\hline algeria & 9476 & 1155 & 8,208 & 0,000 & **** & 10118 & 3336 & 3,033 & 0,002 & **** \\
\hline andean & 11498 & 663,9 & 17,32 & 0,000 & *** & 11477 & 1609 & 7,132 & 0,000 & **** \\
\hline angola & 9549 & 1077 & 8,868 & 0,000 & **** & 10191 & 3054 & 3,337 & 0,001 & **** \\
\hline antigua & 9911 & 990,3 & 10,01 & 0,000 & **** & 10765 & 1876 & 5,738 & 0,000 & **** \\
\hline argentina & 10403 & 517,0 & 20,12 & 0,000 & $* * *$ & 10870 & 1081 & 10,05 & 0,000 & *** \\
\hline armenia & 9059 & 913,3 & 9,918 & 0,000 & **** & 9425 & 1646 & 5,727 & 0,000 & **** \\
\hline asean & 13576 & 1010 & 13,44 & 0,000 & **** & 13687 & 1926 & 7,108 & 0,000 & **** \\
\hline asiaeast & 26670 & 5008 & 5,325 & 0,000 & $* * *$ & 27519 & 13156 & 2,092 & 0,037 & ** \\
\hline asiasoutheast & 31795 & 1666 & 19,09 & 0,000 & **** & 32563 & 4242 & 7,676 & 0,000 & $* * *$ \\
\hline assseasia & 19242 & 2070 & 9,298 & 0,000 & **** & 20792 & 4796 & 4,335 & 0,000 & **** \\
\hline ausnzl & 10035 & 519,1 & 19,33 & 0,000 & **** & 10611 & 992,2 & 10,69 & 0,000 & *** \\
\hline ausnzljpn & 46580 & 736,6 & 63,24 & 0,000 & **** & 47227 & 1526 & 30,94 & 0,000 & **** \\
\hline australia & 4819 & 1031 & 4,677 & 0,000 & **** & 6588 & 1578 & 4,175 & 0,000 & **** \\
\hline austria & $1305 x$ & 615,9 & 21,20 & 0,000 & *** & 12885 & 876,5 & 14,70 & 0,000 & **** \\
\hline azerbaijan & 9061 & 913,3 & 9,921 & 0,000 & **** & 9426 & 1646 & 5,728 & 0,000 & **** \\
\hline bahamas & 9079 & 913,2 & 9,942 & 0,000 & *** & 9442 & 1645 & 5,739 & 0,000 & **** \\
\hline bahrain & 9908 & 990,3 & 10,00 & 0,000 & ***** & 10761 & 1876 & 5,736 & 0,000 & **** \\
\hline bangladesh & 7974 & 624,6 & $12,7 x$ & 0,000 & ***** & 7932 & 1314 & 6,036 & 0,000 & **** \\
\hline barbados & $954 \pi$ & 1080 & 8,839 & 0,000 & *** & 10188 & 3072 & 3,316 & 0,001 & *** \\
\hline belarus & $-2150 x$ & 3391 & $-6,342$ & 0,000 & **** & -18758 & 7197 & $-2,606$ & 0,009 & **** \\
\hline belgium & 13661 & 576,9 & 23,68 & 0,000 & **** & 12947 & 814,7 & 15,89 & 0,000 & **** \\
\hline belgiumlux & 10863 & 887,2 & 12,24 & 0,000 & **** & 12143 & 1103 & 11,01 & 0,000 & **** \\
\hline belize & 9555 & 1084 & 8,816 & 0,000 & *** & 10196 & 3089 & 3,301 & 0,001 & $* * *$ \\
\hline benin & $954 \pi$ & 1079 & 8,845 & 0,000 & $* * *$ & 10189 & 3065 & 3,325 & 0,001 & **** \\
\hline bolivia & 9537 & 1075 & 8,871 & 0,000 & $* * *$ & 10181 & 3053 & 3,334 & 0,001 & **** \\
\hline botswana & 13175 & 719,6 & 18,31 & 0,000 & ***** & 13763 & 1596 & 8,621 & 0,000 & **** \\
\hline brazil & 9598 & 647,2 & 14,83 & 0,000 & **** & 10117 & 1037 & 9,758 & 0,000 & **** \\
\hline brunei & 9545 & 1076 & 8,869 & 0,000 & ***** & 10187 & 3052 & 3,337 & 0,001 & **** \\
\hline bulgaria & 9802 & 810,5 & 12,09 & 0,000 & **** & 10805 & 1772 & 6,097 & 0,000 & ***** \\
\hline burkinafaso & 9544 & 1074 & 8,887 & 0,000 & **** & 10187 & 3046 & 3,344 & 0,001 & *** \\
\hline burundi & 9544 & 1067 & 8,948 & 0,000 & *** & 10188 & 3020 & 3,373 & 0,001 & **** \\
\hline c & 3210 & 1574 & 2,040 & 0,041 & *** & 3120 & 4094 & 0,762 & 0,446 & \\
\hline cairns & 8744 & 601,1 & 14,55 & 0,000 & **** & 9113 & 1075 & 8,480 & 0,000 & *** \\
\hline cairnsdeveloped & $-919,4$ & 828,5 & $-1,110$ & 0,267 & & -1154 & 1335 & $-0,865$ & 0,387 & \\
\hline cairnsdeveloping & -3771 & 651,5 & $-5,789$ & 0,000 & $* * *$ & -3059 & 954,7 & $-3,204$ & 0,001 & **** \\
\hline cambodia & 9063 & 913,3 & 9,924 & 0,000 & **** & 9429 & 1646 & 5,730 & 0,000 & **** \\
\hline cameroon & 12683 & 991,7 & 12,79 & 0,000 & $* * *$ & 13657 & 1913 & 7,138 & 0,000 & *** \\
\hline canada & 7812 & 657,7 & 11,88 & 0,000 & **** & 9159 & 914,0 & 10,02 & 0,000 & **** \\
\hline capeverde & 9073 & 913,2 & 9,936 & 0,000 & **** & 9439 & 1645 & 5,737 & 0,000 & **** \\
\hline carribean & 10359 & 1587 & 6,529 & 0,000 & $* * *$ & 10711 & 4234 & 2,530 & 0,011 & ** \\
\hline $\operatorname{ccs}$ & 5829 & 11952 & 0,488 & 0,626 & & & & & & \\
\hline ceec & 12342 & 1036 & 11,91 & 0,000 & $* * *$ & 12426 & 2619 & 4,745 & 0,000 & **** \\
\hline centralafrrep & 9548 & 1078 & 8,858 & 0,000 & **** & 10190 & 3059 & 3,332 & 0,001 & **** \\
\hline centralamerica & 12360 & 492,0 & 25,12 & 0,000 & **** & 12821 & 1131 & 11,34 & 0,000 & *** \\
\hline cerntraleurope & -9283 & 4878 & $-1,903$ & 0,057 & * & & & & & \\
\hline chad & 9549 & 1077 & 8,868 & 0,000 & $* * *$ & 10191 & 3054 & 3,337 & 0,001 & **** \\
\hline chile & 5518 & 1551 & 3,558 & 0,000 & **** & 9380 & 1520 & 6,171 & 0,000 & **** \\
\hline china & 13804 & 712,0 & 19,39 & 0,000 & **** & 14851 & 974,2 & 15,24 & 0,000 & **** \\
\hline chinahk & 6179 & 1969 & 3,138 & 0,002 & **** & 8338 & 2189 & 3,808 & 0,000 & **** \\
\hline
\end{tabular}




\begin{tabular}{|c|c|c|c|c|c|c|c|c|c|c|}
\hline \multirow{3}{*}{$\begin{aligned} \text { Gewichtung } \rightarrow \\
\text { Stichprobe } \rightarrow \\
\text { Dummy }\end{aligned}$} & \multicolumn{10}{|c|}{ Gewichtet mit [1/(Beobachtungen in der Studie)] } \\
\hline & \multicolumn{5}{|c|}{ mit $B+L^{*}$} & \multicolumn{5}{|c|}{ ohne $B+L^{\circ}$} \\
\hline & Koeff. & Std. Fehl. & t-Went & $\operatorname{Pr}(>|t|)$ & & Koeff. & Std. Fehl. & t-Went & $\operatorname{Pr}(>|t|)$ & \\
\hline chinahks & 11658 & 715,0 & 16,31 & 0,000 & $* * *$ & 12241 & 1128 & 10,85 & 0,000 & *** \\
\hline colombia & 12641 & 875,7 & 14,44 & 0,000 & **** & 12634 & 2152 & 5,870 & 0,000 & *** \\
\hline comoros & 9075 & 913,2 & 9,937 & 0,000 & $* * *$ & 9441 & 1645 & 5,738 & 0,000 & **\# \\
\hline congo & 9541 & 1077 & 8,858 & 0,000 & $* * *$ & 10184 & 3058 & 3,330 & 0,001 & *** \\
\hline congodemrep & 9631 & 1053 & 9,150 & 0,000 & $* * *$ & 10273 & 2874 & 3,575 & 0,000 & *** \\
\hline costarica & 9555 & 1067 & 8,956 & 0,000 & **** & 10199 & 3028 & 3,368 & 0,001 & \\
\hline cotedivore & 9520 & 1062 & 8,967 & 0,000 & **** & 10169 & 3010 & 3,378 & 0,001 & **** \\
\hline croatia & 1354 & 1203 & 1,125 & 0,260 & & 1672 & 1250 & 1,337 & 0,181 & \\
\hline csamerica & -3647 & 6145 & $-0,593$ & 0,553 & & & & & & \\
\hline cuba & 9547 & 1078 & 8,859 & 0,000 & **** & 10189 & 3058 & 3,332 & 0,001 & *** \\
\hline cyprus & 9621 & 1009 & 9,537 & 0,000 & $* * *$ & 10264 & 2778 & 3,694 & 0,000 & *** \\
\hline cyprusmalta & 10880 & 823,5 & 13,21 & 0,000 & **** & 11828 & 1105 & 10,70 & 0,000 & $* *$ \\
\hline czech & 9993 & 821,3 & 12,17 & 0,000 & **** & 10899 & 1853 & 5,883 & 0,000 & *** \\
\hline des & 91454 & 65600 & 1,394 & 0,163 & & 44757 & 50831 & 0,881 & 0,379 & \\
\hline denmark & 13252 & 641,0 & 20,67 & 0,000 & $* * *$ & 13075 & 927,3 & 14,10 & 0,000 & ***** \\
\hline devasia & 16522 & $100 \pi$ & 16,41 & 0,000 & $* * *$ & 16786 & 2211 & 7,591 & 0,000 & *** \\
\hline developed & 19338 & 1713 & 11,29 & 0,000 & $* * *$ & 21119 & 1758 & 12,01 & 0,000 & $* * *$ \\
\hline developedas & 1726 & 3903 & 0,442 & 0,658 & & 34,46 & 6376 & 0,005 & 0,996 & \\
\hline developing & 10146 & 965,3 & 10,51 & 0,000 & $* * *$ & 11252 & 1376 & 8,177 & 0,000 & ***** \\
\hline djibouti & $954 \pi$ & 1079 & 8,849 & 0,000 & **** & 10189 & 3063 & $3,32 \pi$ & 0,001 & *** \\
\hline dominica & 9550 & 982,7 & 9,718 & 0,000 & $* * *$ & 10192 & 2449 & 4,161 & 0,000 & $* * *$ \\
\hline drit & 5509 & 1655 & 3,328 & 0,001 & **** & 5540 & 4319 & 1,283 & 0,200 & \\
\hline eastasean & 13618 & 2000 & 6,808 & 0,000 & **** & 12027 & 3817 & 3,151 & 0,002 & **** \\
\hline eastasia & 9883 & 1054 & 9,380 & 0,000 & $* * *$ & 10721 & 1214 & 8,834 & 0,000 & ***** \\
\hline easternas & 11188 & 552,8 & 20,24 & 0,000 & **** & 11404 & 1218 & 9,367 & 0,000 & *** \\
\hline eba & 10148 & 851,3 & 11,92 & 0,000 & **** & 10281 & 1091 & 9,423 & 0,000 & *** \\
\hline ece & 4843 & 1234 & 3,924 & 0,000 & *** & 6182 & 1356 & 4,559 & 0,000 & *** \\
\hline ecuador & 9546 & 1068 & 8,940 & 0,000 & $* * *$ & 10190 & 3028 & 3,366 & 0,001 & *** \\
\hline ee & 6272 & 546,5 & 11,48 & 0,000 & $* * *$ & 6468 & 964,8 & 6,704 & 0,000 & *4* \\
\hline eec15 & 7985 & 1635 & 4,883 & 0,000 & $* * *$ & 9317 & 2711 & 3,437 & 0,001 & **** \\
\hline eeca & 12416 & 518,3 & 23,96 & 0,000 & $* * *$ & 13368 & 944,0 & 14,16 & 0,000 & *** \\
\hline eecas & 8328 & 426,3 & 19,54 & 0,000 & $* * *$ & 9122 & 758,4 & 12,03 & 0,000 & **** \\
\hline efta & 9487 & 594,6 & 15,96 & 0,000 & $* * *$ & 9770 & 1325 & 7,374 & 0,000 & **** \\
\hline egypt & 9498 & 1019 & 9,324 & 0,000 & **** & 10156 & 2856 & 3,556 & 0,000 & *** \\
\hline eit & 7067 & 1089 & 6,489 & 0,000 & $* * *$ & 8175 & 2220 & 3,682 & 0,000 & **** \\
\hline elsalvador & 9540 & $107 \pi$ & 8,859 & 0,000 & $* * *$ & 10182 & 3064 & 3,323 & 0,001 & *** \\
\hline emin & 9570 & 1058 & \begin{tabular}{|l|}
9,044 \\
\end{tabular} & 0,000 & **** & 10211 & 2985 & 3,421 & 0,001 & **** \\
\hline eritrea & 9071 & 913,2 & 9,933 & 0,000 & $* * *$ & 9437 & 1645 & 5,736 & 0,000 & **** \\
\hline estonia & 10434 & 939,7 & 11,10 & 0,000 & $* * *$ & 11264 & 2289 & 4,920 & 0,000 & **** \\
\hline ethiopia & 9057 & 913,4 & 9,916 & 0,000 & $* * *$ & 9423 & 1646 & 5,726 & 0,000 & **** \\
\hline eulo & 2404 & 913,0 & 2,633 & 0,008 & $* *$ & 2705 & 2161 & 1,252 & 0,211 & \\
\hline eul2 & 24884 & 3307 & 7,525 & 0,000 & $* * *$ & 27869 & 8104 & 3,439 & 0,001 & $* * *$ \\
\hline eul4 & -4760 & 4238 & $-1,123$ & 0,261 & & -2719 & 4439 & $-0,612$ & 0,540 & \\
\hline eul5 & 17362 & 1084 & 16,02 & 0,000 & $* * *$ & 18052 & 1221 & 14,79 & 0,000 & ***4 \\
\hline eul5efta & 75771 & 37254 & 2,034 & 0,042 & $* *$ & 16326 & 6355 & 2,569 & 0,010 & ** \\
\hline eu 25 & 16510 & 2431 & 6,791 & 0,000 & $* * *$ & 17467 & 5734 & 3,046 & 0,002 & $* * *$ \\
\hline eu25ef & 22787 & 4125 & 5,525 & 0,000 & **** & 25321 & 8318 & 3,044 & 0,002 & $* * *$ \\
\hline eu26 & -17178 & 11410 & $-1,506$ & 0,132 & & -8311 & 10832 & $-0,767$ & 0,443 & \\
\hline eure & 14257 & 1047 & 13,62 & 0,000 & $* * *$ & 14902 & 1911 & 7,799 & 0,000 & *** \\
\hline eurest & 15289 & 3147 & 4,858 & 0,000 & $* * *$ & 16528 & 8100 & 2,040 & 0,041 & $* *$ \\
\hline europere & 10729 & 751,7 & 14,27 & 0,000 & $* * *$ & 11410 & 1429 & 7,982 & 0,000 & +4 \\
\hline fiji & 9531 & 1104 & 8,637 & 0,000 & $* * *$ & 10173 & 3156 & \begin{tabular}{|l|}
3,223 \\
\end{tabular} & 0,001 & $* * 4$ \\
\hline finla & 12915 & 631,7 & 20,45 & 0,000 & $* * *$ & 12728 & 905,2 & 14,06 & 0,000 & **** \\
\hline france & 8816 & 1023 & 8,620 & 0,000 & $* * *$ & 9175 & 2279 & $4,02 \pi$ & 0,000 & *** \\
\hline
\end{tabular}




\begin{tabular}{|c|c|c|c|c|c|c|c|c|c|c|}
\hline \multirow{3}{*}{\begin{tabular}{|l|} 
Gewichtung $\rightarrow$ \\
Stichprobe $\rightarrow$ \\
Dummy
\end{tabular}} & \multicolumn{10}{|c|}{ Gewichtet mit [1/(Beobachtungen in der Studie)] } \\
\hline & \multicolumn{5}{|c|}{ mit $\mathbf{B}+\mathrm{L}^{\mathbf{a}}$} & \multicolumn{5}{|c|}{ ohne B+L" } \\
\hline & Koeff. & Std. Fehl. & $t$-Wert & $\operatorname{Pr}(>|t|) \mid$ & & Koeff. & Std. Fehl. & $\mathrm{t}$-Wert & $\operatorname{Pr}(>|t|)$ & \\
\hline frenchpoly & 9071 & 913,2 & 9,933 & 0,000 & ***** & 9437 & 1645 & 5,736 & 0,000 & *** \\
\hline fsu & 1298 & 903,2 & 1,437 & 0,151 & & 1796 & 2134 & 0,842 & 0,400 & \\
\hline gabon & 9546 & 1079 & 8,846 & 0,000 & *** & 10188 & 3063 & 3,326 & 0,001 & **** \\
\hline gambia & 9762 & 670,2 & $14,5 x$ & 0,000 & **** & 10588 & 869,5 & 12,18 & 0,000 & **** \\
\hline georgia & 9055 & 913,4 & 9,913 & 0,000 & *** & 9421 & 1646 & 5,723 & 0,000 & *** \\
\hline germany & 7768 & 948,2 & 8,193 & 0,000 & **** & 8461 & 1676 & 5,049 & 0,000 & **** \\
\hline ghana & 9544 & 1081 & 8,829 & 0,000 & **** & 10186 & 3070 & 3,318 & 0,001 & **** \\
\hline greatbritain & 11969 & 1101 & 10,87 & 0,000 & **** & 12478 & 1860 & 6,710 & 0,000 & **** \\
\hline greece & 12671 & 680,6 & 18,62 & 0,000 & **** & 12499 & 1007 & 12,41 & 0,000 & **** \\
\hline grenada & 9549 & 1074 & 8,891 & 0,000 & **** & 10190 & 3049 & 3,342 & 0,001 & \\
\hline grouptwenty & $-208,7$ & 3182 & $-0,066$ & 0,948 & & 2838 & 2081 & 1,364 & 0,173 & \\
\hline guatemala & 9556 & 1070 & 8,928 & 0,000 & **** & 10197 & 3035 & 3,360 & 0,001 & **** \\
\hline guinea & 9548 & 1078 & 8,860 & 0,000 & **** & 10190 & 3058 & 3,332 & 0,001 & **** \\
\hline guineabissau & 9548 & 1078 & 8,860 & 0,000 & ***** & 10190 & 3058 & 3,332 & 0,001 & **** \\
\hline guyana & 9541 & 1094 & 8,725 & 0,000 & **** & 10184 & 3117 & 3,267 & 0,001 & **** \\
\hline haiti & 9545 & 1081 & 8,834 & 0,000 & $* * *$ & 10187 & 3071 & 3,317 & 0,001 & **** \\
\hline hiasia & 17826 & 1518 & 11,74 & 0,000 & $* * *$ & 18776 & 2647 & 7,093 & 0,000 & **** \\
\hline highincome & 142896 & 16081 & 8,886 & 0,000 & $* * *$ & 155239 & 39808 & 3,900 & 0,000 & $* * *$ \\
\hline hktaikorea & 20947 & 1781 & 11,76 & 0,000 & $* * *$ & 21799 & 4417 & 4,936 & 0,000 & **** \\
\hline hktaising & 14620 & 644,3 & 22,69 & 0,000 & **** & 14457 & 1192 & 12,13 & 0,000 & **** \\
\hline honduras & 9538 & 1066 & 8,946 & 0,000 & **** & 10182 & 3028 & 3,363 & 0,001 & **** \\
\hline hongkong & 2969 & 1671 & 1,777 & 0,076 & * & 7121 & 1586 & 4,490 & 0,000 & **** \\
\hline hongkongsing & 9030 & 1176 & 7,677 & 0,000 & $* * *$ & 9585 & 2948 & 3,251 & 0,001 & *** \\
\hline hungary & 9885 & 798,5 & 12,38 & 0,000 & **** & 10778 & 1727 & 6,240 & 0,000 & **** \\
\hline iceland & 9588 & 1038 & 9,234 & 0,000 & **** & 10230 & 2901 & 3,526 & 0,000 & **** \\
\hline india & 7460 & 783,1 & 9,526 & 0,000 & **** & 8243 & 1084 & 7,603 & 0,000 & **** \\
\hline indonesia & 7843 & 1087 & 7,216 & 0,000 & **** & 10610 & 1682 & 6,308 & 0,000 & *** \\
\hline iran & 9326 & 1029 & 9,065 & 0,000 & **** & 10008 & 2945 & 3,398 & 0,001 & **** \\
\hline iraq & 9007 & 916,0 & 9,832 & 0,000 & **** & 9370 & 1657 & 5,656 & 0,000 & **** \\
\hline ireland & 11901 & 702,4 & 16,94 & 0,000 & **** & 11861 & 1268 & 9,356 & 0,000 & **** \\
\hline israel & 9761 & 917,3 & 10,64 & 0,000 & **** & 10403 & 2299 & 4,525 & 0,000 & **** \\
\hline italy & 9679 & 1229 & 7,874 & 0,000 & *** & 10285 & 1881 & 5,467 & 0,000 & **** \\
\hline jamaica & 9522 & 1088 & 8,752 & 0,000 & $* * *$ & 10166 & 3105 & 3,275 & 0,001 & **** \\
\hline japan & 15783 & 2283 & 6,912 & 0,000 & **** & 15301 & 1616 & 9,466 & 0,000 & **** \\
\hline japankorea & 22020 & 2565 & 8,584 & 0,000 & **** & 22364 & 6635 & 3,370 & 0,001 & **** \\
\hline jordan & 4947 & 1411 & 3,507 & 0,000 & **** & 2814 & 1705 & 1,650 & 0,099 & * \\
\hline kazakhstan & -22000 & 2533 & $-8,684$ & 0,000 & $* * *$ & -19213 & 4614 & $-4,164$ & 0,000 & *** \\
\hline kenya & 9066 & 913,6 & 9,924 & 0,000 & $* * *$ & 9434 & 1646 & 5,731 & 0,000 & **** \\
\hline korea & 11618 & 1348 & 8,619 & 0,000 & **** & 14530 & 1609 & 9,033 & 0,000 & **** \\
\hline koreadpr & 9057 & 913,3 & 9,916 & 0,000 & $* * *$ & 9422 & 1646 & 5,725 & 0,000 & **** \\
\hline koreataiwan & 20797 & 2180 & 9,538 & 0,000 & **** & 21766 & 5583 & 3,899 & 0,000 & **** \\
\hline kuwait & 9577 & 1050 & 9,122 & 0,000 & **** & 10218 & 2953 & 3,460 & 0,001 & **** \\
\hline kyrgyzstan & 9551 & 1075 & 8,887 & 0,000 & **** & 10193 & $304 x$ & 3,345 & 0,001 & **** \\
\hline laos & 9076 & 913,2 & 9,938 & 0,000 & **** & 9442 & 1645 & 5,739 & 0,000 & **** \\
\hline latin & 12689 & 914,3 & 13,88 & 0,000 & **** & 13517 & 1695 & 7,976 & 0,000 & **** \\
\hline latvia & 10390 & 928,9 & 11,18 & 0,000 & $* * *$ & 11222 & 2252 & 4,982 & 0,000 & **** \\
\hline leastdeveloped & 10145 & 1458 & 6,958 & 0,000 & *** & 10012 & 1930 & 5,186 & 0,000 & **** \\
\hline lebanon & 9089 & 913,2 & 9,953 & 0,000 & *** & 9453 & 1645 & 5,746 & 0,000 & **** \\
\hline lesotho & 9089 & 916,8 & 9,914 & 0,000 & **** & 9455 & 1659 & 5,699 & 0,000 & **** \\
\hline liaexp & 5981 & 494,8 & 12,09 & 0,000 & **** & 6324 & 1111 & 5,692 & 0,000 & **** \\
\hline liberia & 9072 & 913,2 & 9,935 & 0,000 & *** & 9438 & 1645 & 5,737 & 0,000 & **** \\
\hline libya & 9056 & 913,5 & 9,914 & 0,000 & $* * *$ & 9423 & 1646 & 5,724 & 0,000 & **** \\
\hline liexp & 5495 & 1346 & 4,081 & 0,000 & **** & 6289 & 2576 & 2,441 & 0,015 & ** \\
\hline $\operatorname{limp}$ & 8425 & 637,1 & 13,22 & 0,000 & *** & 8692 & 1619 & 5,370 & 0,000 & **** \\
\hline
\end{tabular}




\begin{tabular}{|c|c|c|c|c|c|c|c|c|c|c|}
\hline \multirow{3}{*}{$\begin{array}{c}\text { Gewichtung } \rightarrow \\
\text { Stichprobe } \rightarrow \\
\text { Dummy }\end{array}$} & \multicolumn{10}{|c|}{ Gewichtet mit [1/(Beobachtungen in der Studie)] } \\
\hline & \multicolumn{5}{|c|}{ mit $B+L^{*}$} & \multicolumn{5}{|c|}{ ohne B+L" } \\
\hline & Koeff. & Std. Fehl. & $t$-Wert & $\operatorname{Pr}(>|t|)$ & & Koeff. & Std. Fehl. & $t$-Wert & $\operatorname{Pr}(>|t|)$ & \\
\hline lithuania & 10268 & 887,6 & 11,57 & 0,000 & *** & 11106 & 2101 & 5,286 & 0,000 & *** \\
\hline Imiimp & 6452 & 637,1 & 10,13 & 0,000 & *** & 7028 & 1212 & 5,798 & 0,000 & *** \\
\hline lowincom & 3139 & 6773 & 0,463 & 0,643 & & 10678 & 3440 & 3,104 & 0,002 & *** \\
\hline macao & 9547 & 1079 & 8,849 & 0,000 & *** & 10189 & 3063 & $3,32 \pi$ & 0,001 & *** \\
\hline macedonia & 9072 & 913,2 & 9,934 & 0,000 & $* * *$ & 9438 & 1645 & 5,737 & 0,000 & ****4 \\
\hline madagascar & 14105 & 2974 & 4,743 & 0,000 & *** & 14795 & 7934 & 1,865 & 0,062 & * \\
\hline maghreb & 17832 & 747,8 & 23,85 & 0,000 & $* *$ & 17693 & 1561 & 11,33 & 0,000 & $* * *$ \\
\hline malawi & 11083 & 530,4 & 20,90 & 0,000 & $* * *$ & 11774 & 911,6 & 12,92 & 0,000 & $* * *$ \\
\hline malaysia & 4110 & 1664 & 2,470 & 0,014 & ** & 8745 & 1624 & 5,386 & 0,000 & *** \\
\hline maledives & 9546 & 1077 & 8,865 & 0,000 & $* * *$ & 10188 & 3055 & 3,335 & 0,001 & *** \\
\hline mali & 9541 & 1069 & 8,926 & 0,000 & **** & 10186 & 3029 & 3,362 & 0,001 & *** \\
\hline malta & 9068 & 913,2 & 9,930 & 0,000 & *** & 9434 & 1645 & 5,734 & 0,000 & *** \\
\hline marrocco & 9073 & 2198 & 4,128 & 0,000 & *** & 13126 & 1796 & 7,308 & 0,000 & $* * *$ \\
\hline mauritania & 9545 & 1081 & 8,830 & 0,000 & **** & 10187 & 3071 & 3,318 & 0,001 & \\
\hline mauritius & 9517 & 1121 & 8,488 & 0,000 & **** & 10160 & 3213 & 3,162 & 0,002 & *** \\
\hline mediterranean & 6592 & 1755 & 3,75 ) & 0,000 & **** & 6780 & 4437 & 1,528 & $0,12 \pi$ & \\
\hline mena & 9064 & 849,7 & 10,67 & 0,000 & *** & 9497 & 2119 & 4,482 & 0,000 & *** \\
\hline mer & 4439 & 1325 & 3,351 & 0,001 & **** & 5903 & 1373 & 4,301 & 0,000 & *** \\
\hline mercosur & 11083 & 813,5 & 13,62 & 0,000 & **** & 11492 & 1897 & 6,058 & 0,000 & *** \\
\hline mexico & 7125 & 874,0 & 8,153 & 0,000 & **** & 9208 & 1079 & 8,535 & 0,000 & **** \\
\hline miaexp & 5751 & 856,9 & 6,711 & 0,000 & **** & 6654 & 1224 & 5,437 & 0,000 & *** \\
\hline middleea & 11740 & 692,0 & 16,97 & 0,000 & **** & 12214 & 1599 & 7,641 & 0,000 & *** \\
\hline middleincome & 125352 & 13128 & 9,548 & 0,000 & *** & 137535 & 18238 & 7,541 & 0,000 & *** \\
\hline miexp & 10779 & 1194 & 9,031 & 0,000 & **** & 11701 & 3072 & 3,809 & 0,000 & *** \\
\hline mifexp & 13059 & 2115 & 6,175 & 0,000 & **** & 13673 & 5778 & 2,366 & 0,018 & ** \\
\hline $\mathrm{mna}$ & 5388 & 955,8 & 5,637 & 0,000 & **** & 6664 & 1702 & 3,915 & 0,000 & *** \\
\hline moldova & 9078 & 913,2 & 9,941 & 0,000 & $* * *$ & 9444 & 1645 & 5,740 & 0,000 & $* * *$ \\
\hline mongolia & 9910 & 990,3 & 10,01 & 0,000 & $* * *$ & 10764 & 1876 & $5,73 \pi$ & 0,000 & $* * *$ \\
\hline mozambiqu & 12155 & 514,2 & 23,64 & 0,000 & **** & 12553 & $107 x$ & 11,65 & 0,000 & *** \\
\hline myanmar & 9910 & 990,3 & 10,01 & 0,000 & $* * *$ & 10764 & 1876 & $5,73 \pi$ & 0,000 & $* * *$ \\
\hline nafta & 9759 & 2505 & 3,896 & 0,000 & $* * *$ & 11041 & 5874 & 1,880 & 0,060 & * \\
\hline namibia & 9085 & 913,2 & 9,948 & 0,000 & **** & 9451 & 1645 & 5,744 & 0,000 & $* * *$ \\
\hline nea & 14941 & 920,7 & 16,23 & 0,000 & $* * *$ & 15605 & 1716 & 9,095 & 0,000 & $* * *$ \\
\hline nepal & 9070 & 913,2 & 9,931 & 0,000 & $* * *$ & 9436 & 1645 & 5,735 & 0,000 & $* * *$ \\
\hline nethant & 9071 & 913,2 & 9,934 & 0,000 & **** & 9437 & 1645 & 5,736 & 0,000 & $* * *$ \\
\hline netherland & 6494 & 664,3 & 9,776 & 0,000 & $* * *$ & 6654 & 1412 & 4,714 & 0,000 & $* * *$ \\
\hline nic & 5657 & 967,5 & 5,846 & 0,000 & $* * *$ & 6683 & 1106 & 6,045 & 0,000 & ***4 \\
\hline nicaragua & 9551 & 1069 & 8,933 & 0,000 & $* * *$ & 10192 & 3033 & 3,360 & 0,001 & $* * *$ \\
\hline nicasia & 11830 & 982,9 & 12,04 & 0,000 & $* * *$ & 12603 & 1349 & 9,345 & 0,000 & 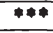 \\
\hline niger & 9546 & 1079 & 8,846 & 0,000 & $* * *$ & 10188 & 3064 & 3,326 & 0,001 & $* * *$ \\
\hline nigeria & 9735 & 638,4 & 15,25 & 0,000 & $* * *$ & 10577 & 845,6 & 12,51 & 0,000 & *** \\
\hline nis & 6502 & 988,6 & 6,577 & 0,000 & $* * *$ & 7396 & 1333 & 5,547 & 0,000 & $* * *$ \\
\hline $\mathrm{nmb}$ & 11144 & 1467 & 7,595 & 0,000 & 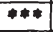 & 14123 & 1393 & 10,14 & 0,000 & $* * *$ \\
\hline northafrica & 10874 & 984,0 & 11,05 & 0,000 & $* * *$ & 11183 & 1481 & 7,551 & 0,000 & ***** \\
\hline northamerica & 14285 & 1196 & 11,94 & 0,000 & $* * *$ & 14389 & 2959 & 4,863 & 0,000 & 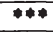 \\
\hline northeastasia & 27793 & 1606 & 17,31 & 0,000 & **** & 28214 & 4079 & 6,916 & 0,000 & **** \\
\hline norway & 9855 & 895,5 & 11,00 & 0,000 & ****1\% & 10496 & 2052 & 5,115 & 0,000 & **** \\
\hline $\mathrm{nzl}$ & 4556 & 1065 & 4,276 & 0,000 & $* * *$ & 6765 & 1640 & 4,124 & 0,000 & $* * *$ \\
\hline oasia & 12413 & 619,6 & 20,03 & 0,000 & **** & 13267 & 1503 & 8,825 & 0,000 & ***** \\
\hline oasp & 14952 & 880,2 & 16,99 & 0,000 & $* * *$ & 14986 & 2194 & 6,830 & 0,000 & $* * *$ \\
\hline oaspa & 6372 & 6148 & 1,037 & 0,300 & & 6180 & 15892 & 0,389 & 0,697 & \\
\hline oaspac & 8109 & 1083 & 7,486 & 0,000 & *** & 7951 & 2577 & 3,085 & 0,002 & $* * *$ \\
\hline ocd & 1933 & 1889 & 1,023 & 0,306 & & 4059 & 1506 & 2,695 & 0,007 & $* * *$ \\
\hline oceania & 14038 & 542,4 & 25,88 & 0,000 & *** & 14205 & 1174 & 12,10 & 0,000 & **** \\
\hline
\end{tabular}




\begin{tabular}{|c|c|c|c|c|c|c|c|c|c|c|}
\hline \multirow{3}{*}{\begin{tabular}{|c|} 
Gewichtung $\rightarrow$ \\
Stichprobe $\rightarrow$ \\
Dummy
\end{tabular}} & \multicolumn{10}{|c|}{ Gewichtet mit [1/(Beobachtungen in der Studie)] } \\
\hline & \multicolumn{5}{|c|}{ mit $B+L^{*}$} & \multicolumn{5}{|c|}{ ohne $B+L^{\circ}$} \\
\hline & Koeff. & Std. Fehl. & $\mathrm{t}$-Wert & $\operatorname{Pr}(>|t|) \mid$ & & Koeff. & Std. Fehl. & t-Wert & $\operatorname{Pr}(>|t|) \mid$ & \\
\hline oeasia & 13156 & 559,3 & 23,52 & 0,000 & **** & 12946 & 1074 & 12,06 & 0,000 & *\#* \\
\hline oeefsu & 10539 & 676,7 & 15,57 & 0,000 & **** & 10995 & 1438 & 7,647 & 0,000 & *** \\
\hline oeurope & 9716 & 1588 & 6,117 & 0,000 & **** & 11701 & 3269 & 3,580 & 0,000 & **** \\
\hline olac & 7533 & 815,8 & 9,233 & 0,000 & **** & 8368 & 1422 & 5,884 & 0,000 & **** \\
\hline olat & 8571 & 949,9 & 9,023 & 0,000 & **** & 8914 & 2241 & 3,978 & 0,000 & **** \\
\hline oman & 9907 & 990,3 & 10,00 & 0,000 & ***** & 10760 & 1876 & 5,735 & 0,000 & **** \\
\hline onam & 12777 & $602, \pi$ & 21,20 & 0,000 & **** & 12724 & 1312 & 9,702 & 0,000 & *** \\
\hline osea & 11756 & 728,9 & 16,13 & 0,000 & *** & 12436 & 1681 & 7,396 & 0,000 & *** \\
\hline othapec & 6519 & 1041 & 6,264 & 0,000 & $* * *$ & 7103 & 1233 & 5,763 & 0,000 & *** \\
\hline othasia & 10983 & 728,8 & 15,07 & 0,000 & *\#* & 11186 & 1768 & 6,327 & 0,000 & **** \\
\hline othdeveloped & 6850 & 742,5 & 9,226 & 0,000 & ***** & 7490 & 1516 & 4,941 & 0,000 & *** \\
\hline otheca & 9976 & 790,7 & 12,62 & 0,000 & **** & 10788 & 1109 & 9,729 & 0,000 & *** \\
\hline otherafrica & 9959 & 790,5 & 12,60 & 0,000 & $* * *$ & 10771 & 1108 & 9,722 & 0,000 & *** \\
\hline othhiasia & 8405 & 1237 & 6,793 & 0,000 & **** & 8956 & 1305 & 6,862 & 0,000 & **** \\
\hline othlatin & 11159 & 1199 & 9,306 & 0,000 & **** & 11909 & 2511 & 4,743 & 0,000 & **** \\
\hline othnic & 6344 & 1776 & 3,573 & 0,000 & **** & 7287 & 4250 & 1,714 & 0,087 & * \\
\hline othsea & 9406 & 551,7 & 17,05 & 0,000 & **** & 10108 & 1012 & 9,983 & 0,000 & **** \\
\hline othseasia & 1403 & 2561 & 0,548 & 0,584 & & 2425 & 6265 & 0,387 & 0,699 & \\
\hline othsouthasia & 1335 & 2209 & 0,604 & 0,546 & & 5168 & 4437 & 1,165 & 0,244 & \\
\hline owe & 11752 & 653,2 & 17,99 & 0,000 & **** & 12457 & 1159 & 10,75 & 0,000 & **** \\
\hline pakistan & 9420 & 1021 & 9,222 & 0,000 & **** & 10092 & 2876 & 3,509 & 0,000 & **** \\
\hline panama & 9552 & 1078 & 8,863 & 0,000 & **** & 10193 & 3064 & 3,327 & 0,001 & **** \\
\hline papua & 9551 & 1074 & 8,894 & 0,000 & **** & 10193 & 3044 & 3,349 & 0,001 & $* * *$ \\
\hline paraguay & 9549 & 1076 & 8,871 & 0,000 & **** & 10192 & 3053 & 3,338 & 0,001 & **** \\
\hline peru & 12871 & 789,0 & 16,31 & 0,000 & **** & 12693 & 1799 & 7,054 & 0,000 & $* * *$ \\
\hline philippines & 2911 & 1376 & 2,116 & 0,034 & $* *$ & 5882 & 1813 & 3,245 & 0,001 & **** \\
\hline poland & 8320 & 1018 & 8,173 & 0,000 & **** & 9472 & 1515 & 6,252 & 0,000 & **** \\
\hline portugal & 12718 & 637,5 & 19,95 & 0,000 & **** & 12543 & 912,7 & 13,74 & 0,000 & **** \\
\hline gatar & 9910 & 990,3 & 10,01 & 0,000 & **** & 10763 & 1876 & 5,737 & 0,000 & *** \\
\hline racp & 10325 & 857,4 & 12,04 & 0,000 & **** & 10458 & 1120 & 9,337 & 0,000 & **** \\
\hline rafrica & 11062 & 454,4 & 24,34 & 0,000 & **** & 11324 & 909,5 & 12,45 & 0,000 & **** \\
\hline rasia & 8648 & 2131 & 4,058 & 0,000 & $* * *$ & 13590 & 1290 & 10,54 & 0,000 & $* * *$ \\
\hline rcairns & 9270 & 1804 & 5,139 & 0,000 & **** & 11283 & 1874 & 6,020 & 0,000 & **** \\
\hline rcarribbean & 15095 & 422,5 & 35,73 & 0,000 & **** & 15829 & 751,3 & 21,07 & 0,000 & $* * *$ \\
\hline rdeveloped & 11222 & 541,4 & 20,73 & 0,000 & **** & 11642 & 1046 & 11,13 & 0,000 & $* * *$ \\
\hline rea & 7473 & 1336 & 5,594 & 0,000 & *** & 8016 & 3151 & 2,544 & 0,011 & ** \\
\hline reastasia & 31037 & 8295 & 3,742 & 0,000 & **** & 31816 & 21789 & 1,460 & 0,144 & \\
\hline reca & 2574 & 1359 & 1,895 & 0,058 & $*$ & 3424 & 2607 & 1,313 & 0,189 & \\
\hline refta & 10123 & 829,7 & 12,20 & 0,000 & **** & 10333 & 1132 & 9,125 & 0,000 & **** \\
\hline restasia & 6456 & 412,7 & 15,65 & 0,000 & **** & 7190 & 712,4 & 10,09 & 0,000 & ***** \\
\hline reu & 7339 & 1983 & 3,702 & 0,000 & **** & 8673 & 3088 & 2,809 & 0,005 & **** \\
\hline reurope & -4693 & 2298 & $-2,042$ & 0,041 & *** & -3991 & 2612 & $-1,528$ & 0,127 & \\
\hline rlatin & 6577 & 602,7 & 10,91 & 0,000 & **** & 6999 & 1091 & 6,414 & 0,000 & **** \\
\hline rme & 2511 & 9089 & 0,276 & 0,782 & & & & & & \\
\hline middlefast & 13319 & 761,6 & 17,49 & 0,000 & **** & 13191 & 1463 & 9,013 & 0,000 & **** \\
\hline rnorthafrica & 13185 & 956,6 & 13,78 & 0,000 & **** & 13353 & 2267 & 5,891 & 0,000 & **** \\
\hline roasia & 12026 & 722,7 & 16,64 & 0,000 & **** & 12822 & 1593 & 8,048 & 0,000 & $* * *$ \\
\hline roecd & 13192 & 475,7 & 27,73 & 0,000 & **** & 13246 & 1092 & 12,13 & 0,000 & **** \\
\hline romania & 10945 & 996,7 & 10,98 & 0,000 & **** & 11913 & 2530 & 4,709 & 0,000 & **** \\
\hline row & 17689 & 10160 & 1,741 & 0,082 & * & 10795 & 9255 & 1,166 & 0,243 & \\
\hline rsa & 1135 & 829,1 & 1,369 & 0,171 & & 1637 & 1741 & 0,940 & 0,347 & \\
\hline rsacu & 10729 & 863,8 & 12,42 & 0,000 & **** & 11945 & 1099 & 10,87 & 0,000 & **** \\
\hline rsothasia & 10148 & 668,9 & 15,17 & 0,000 & **** & 10591 & 1608 & 6,588 & 0,000 & **** \\
\hline rsouthafrica & 11059 & 708,5 & 15,61 & 0,000 & $* * *$ & 11610 & 1113 & 10,43 & 0,000 & *** \\
\hline
\end{tabular}




\begin{tabular}{|c|c|c|c|c|c|c|c|c|c|c|}
\hline \multirow{3}{*}{$\begin{array}{l}\text { Gewichtung } \rightarrow \\
\text { Stichprobe } \rightarrow \\
\text { Dummy }\end{array}$} & \multicolumn{10}{|c|}{ Gewichtet mit [1/(Beobachtungen in der Studie)] } \\
\hline & \multicolumn{5}{|c|}{ mit $B+L^{*}$} & \multicolumn{5}{|c|}{ ohne $B+L^{\circ}$} \\
\hline & Koeff. & Std. Fehl. & $t$-Wert & $\operatorname{Pr}(>|t|)$ & & Koeff. & Std. Fehl. & $t$-Wert & $\operatorname{Pr}(>|t|)$ & \\
\hline rsouthamerica & 13487 & 732,3 & 18,42 & 0,000 & *** & 13192 & 1771 & 7,449 & 0,000 & *** \\
\hline rsouthernafrica & 11729 & 689,8 & 17,00 & 0,000 & **** & 11791 & 1598 & 7,378 & 0,000 & **** \\
\hline rssa & 8783 & 913,4 & 9,616 & 0,000 & *** & 9148 & 1646 & 5,558 & 0,000 & **\# \\
\hline rsubsaharan & 7828 & 660,3 & 11,85 & 0,000 & *** & 8463 & 1429 & 5,921 & 0,000 & *** \\
\hline russia & $-18,75$ & 1017 & $-0,018$ & 0,985 & & 1474 & 1569 & 0,940 & 0,347 & \\
\hline rwanda & 9548 & $107 \pi$ & 8,863 & 0,000 & *** & 10190 & 3057 & 3,334 & 0,001 & *** \\
\hline saarc & 9220 & 1042 & 8,847 & 0,000 & $* * *$ & 9886 & 2111 & 4,682 & 0,000 & $* *$ \\
\hline sacu & 7746 & 1209 & 6,405 & 0,000 & $* * *$ & 9635 & 1919 & 5,022 & 0,000 & $* * *$ \\
\hline saf & 8636 & 498,7 & 17,32 & 0,000 & $* * *$ & 8723 & 1060 & 8,227 & 0,000 & *** \\
\hline salomon & 9549 & 1077 & 8,867 & 0,000 & $* * *$ & 10191 & 3055 & 3,336 & 0,001 & $* * *$ \\
\hline saotome & 9075 & 913,2 & 9,938 & 0,000 & **** & 9441 & 1645 & 5,739 & 0,000 & *** \\
\hline saudiarabia & 9487 & 1140 & 8,320 & 0,000 & **** & 10128 & 3292 & 3,076 & 0,002 & **** \\
\hline sea & 13609 & 571,6 & 23,81 & 0,000 & **** & 13973 & 1082 & 12,91 & 0,000 & **** \\
\hline selecteds & 2887 & 711,1 & 4,059 & 0,000 & **** & 3329 & 1456 & 2,286 & 0,022 & ** \\
\hline senegal & 9791 & 670,5 & 14,60 & 0,000 & *** & 10616 & 870,6 & 12,19 & 0,000 & $* *$ \\
\hline seychelles & 9075 & 913,2 & $9,93 x$ & 0,000 & **** & 9441 & 1645 & 5,738 & 0,000 & $* * *$ \\
\hline sierraleone & 9548 & 1078 & 8,859 & 0,000 & **** & 10190 & 3059 & 3,332 & 0,001 & *** \\
\hline singapore & -1280 & 1855 & $-0,690$ & 0,490 & & 2037 & 2535 & 0,803 & 0,422 & \\
\hline slovakia & 10282 & 895,8 & 11,48 & 0,000 & $* * *$ & 11139 & 2150 & 5,180 & 0,000 & *** \\
\hline slovenia & 10279 & 896,3 & 11,47 & 0,000 & *** & 11144 & 2159 & 5,162 & 0,000 & $* * *$ \\
\hline somalia & 9075 & 913,2 & 9,937 & 0,000 & *** & 9441 & 1645 & 5,738 & 0,000 & *** \\
\hline southamerica & 9695 & 1618 & 5,990 & 0,000 & $* * *$ & 9783 & 2079 & 4,705 & 0,000 & \\
\hline southasia & 13208 & 1279 & 10,33 & 0,000 & $* * *$ & 13863 & 3060 & 4,530 & 0,000 & *** \\
\hline southernafr & 11827 & 418,1 & 28,28 & 0,000 & **** & 11790 & 878,6 & 13,42 & 0,000 & *** \\
\hline southerncon & 7832 & 1235 & 6,344 & 0,000 & $* * *$ & 8384 & 1289 & 6,503 & 0,000 & **** \\
\hline spain & $1302 \pi$ & 808,4 & 16,11 & 0,000 & $* * *$ & 13010 & 1213 & 10,72 & 0,000 & $* * *$ \\
\hline srilanka & 7591 & 2468 & 3,075 & 0,002 & $* * *$ & 13606 & 1933 & 7,039 & 0,000 & **** \\
\hline ssa & 7908 & 492,8 & 16,05 & 0,000 & **** & 881 & 1032 & 7,833 & 0,000 & *** \\
\hline ssasacu & 10540 & 580,5 & 18,16 & 0,000 & **** & 11726 & 984,9 & 11,91 & 0,000 & *** \\
\hline stkitts & 9921 & 990,4 & 10,02 & 0,000 & $* * *$ & 10774 & 1877 & 5,742 & 0,000 & $* *$ \\
\hline stlucia & 9562 & 1084 & 8,823 & 0,000 & $* * *$ & 10203 & 3086 & 3,307 & 0,001 & **** \\
\hline stvincen & 9557 & 1079 & 8,854 & 0,000 & **** & 10198 & 3069 & 3,323 & 0,001 & **** \\
\hline sudan & 9537 & 1074 & 8,879 & 0,000 & **** & 81 & 3049 & 3,339 & 0,001 & **** \\
\hline suriname & 9551 & 1081 & 8,839 & 0,000 & *** & 10193 & 3069 & 3,321 & 0,001 & **** \\
\hline swaziland & 9086 & 913,3 & 9,948 & 0,000 & **** & 9452 & 1646 & 5,744 & 0,000 & $* * *$ \\
\hline sweden & 9566 & 1759 & 5,438 & 0,000 & $* * *$ & 10076 & 3062 & 3,291 & 0,001 & **** \\
\hline switzerland & 9224 & 405,0 & 22,77 & 0,000 & **** & 9117 & 793,7 & 11,49 & 0,000 & **** \\
\hline syria & 9074 & 913,2 & 9,937 & 0,000 & **** & $943 x$ & 1645 & 5,735 & 0,000 & $* * *$ \\
\hline$t$ & 19441 & 2342 & 8,301 & 0,000 & **** & 19217 & 5936 & 3,237 & 0,001 & $* *$ \\
\hline taiwan & -3258 & 1161 & $-2,80 x$ & 0,005 & $* * *$ & -1705 & 1360 & $-1,254$ & 0,210 & \\
\hline tajkistan & 9066 & 913,2 & 9,927 & 0,000 & **** & 9432 & 1645 & 5,732 & 0,000 & $* * *$ \\
\hline tanzania & 11028 & 575,7 & 19,16 & 0,000 & $* * *$ & 11448 & 1130 & 10,13 & 0,000 & $* * *$ \\
\hline thaijapkorea & -22483 & 1953 & $-11,51$ & 0,000 & $* * *$ & -19659 & 2295 & $-8,565$ & 0,000 & *** \\
\hline thailand & 7759 & 1311 & 5,917 & 0,000 & $* * *$ & 11405 & 1762 & 6,474 & 0,000 & *** \\
\hline tigers & 14054 & 1013 & 13,87 & 0,000 & $* * *$ & 13803 & 2288 & 6,033 & 0,000 & **** \\
\hline togo & 9545 & 1076 & 8,871 & 0,000 & **** & 10188 & 3053 & 3,337 & 0,001 & $* * *$ \\
\hline trans & 11930 & 475,2 & 25,11 & 0,000 & $* * *$ & 12139 & 932,0 & 13,03 & 0,000 & $* * *$ \\
\hline trinidad & $954 x$ & 1079 & 8,851 & 0,000 & $* * *$ & 10188 & 3066 & 3,323 & 0,001 & *** \\
\hline tuc & 6498 & 780,6 & 8,325 & 0,000 & $* * *$ & 6400 & 1925 & 3,324 & 0,001 & $* * 4$ \\
\hline tunesia & 14351 & 3128 & 4,588 & 0,000 & $* * *$ & 15075 & 8404 & 1,794 & 0,073 & * \\
\hline turkey & $735 \mathrm{~s}$ & 866,9 & 8,484 & 0,000 & $* * *$ & 8979 & 1530 & 5,869 & 0,000 & $* * *$ \\
\hline turkmeni & 9072 & 913,2 & 9,934 & 0,000 & $* * *$ & 9437 & 1645 & 5,736 & 0,000 & *** \\
\hline uganda & 10832 & 547,3 & 19,79 & 0,000 & $* * *$ & 11290 & 1067 & 10,58 & 0,000 & $* * 4$ \\
\hline ukraine & 9164 & 918,4 & 9,979 & 0,000 & $* * *$ & 9535 & 1663 & 5,735 & 0,000 & $* * *$ \\
\hline
\end{tabular}




\begin{tabular}{|c|c|c|c|c|c|c|c|c|c|c|}
\hline Gewichtung $\rightarrow$ & & & wichte & mit $[1 /(1$ & Beob & htungen & in der $\mathrm{St}$ & & & \\
\hline Stichprobe $\rightarrow$ & & mit & $3+L^{2}$ & & & & ohne & B+L & & \\
\hline Dummy & Koeff. & Std. Fehl. & $t$-Wert & $\operatorname{Pr}(>|t|)$ & & Koeff. & Std. Fehl. & $t$-Wert & $\operatorname{Pr}(>|t|)$ & \\
\hline umiimp & 8757 & 767,4 & 11,41 & 0,000 & *** & 9380 & 1918 & 4,892 & 0,000 & **** \\
\hline uruguay & 11236 & 766,9 & 14,65 & 0,000 & **** & 11482 & 1369 & 8,385 & 0,000 & **** \\
\hline usa & 16960 & 2182 & 7,773 & 0,000 & $* * *$ & 13556 & 969,6 & 13,98 & 0,000 & **** \\
\hline usbekistan & 9059 & 913,3 & 9,919 & 0,000 & **** & 9426 & 1645 & 5,730 & 0,000 & **** \\
\hline ussr & 7939 & 1050 & 7,564 & 0,000 & ***** & 9025 & 2073 & 4,353 & 0,000 & $* * *$ \\
\hline vanuatu & 9075 & 913,2 & 9,938 & 0,000 & $* * *$ & 9441 & 1645 & 5,739 & 0,000 & **** \\
\hline venezuela & 12682 & 760,2 & 16,68 & 0,000 & ***** & 12515 & 1716 & 7,291 & 0,000 & **** \\
\hline vietnam & 14068 & 621,9 & 22,62 & 0,000 & ***** & 13724 & 1340 & 10,24 & 0,000 & *** \\
\hline westernel & 20941 & 1757 & 11,92 & 0,000 & **** & 22023 & 3285 & 6,703 & 0,000 & **** \\
\hline yemen & 9011 & 915,1 & 9,848 & 0,000 & ***** & 9376 & 1654 & 5,669 & 0,000 & **** \\
\hline yugoslavia & 9088 & 913,6 & $9,94 \pi$ & 0,000 & ***** & 9457 & 1646 & 5,747 & 0,000 & $* * *$ \\
\hline zambia & 11398 & 523,9 & 21,75 & 0,000 & ***** & 11910 & 1049 & 11,35 & 0,000 & *** \\
\hline zimbabwe & 12784 & 702,6 & 18,20 & 0,000 & **** & 13389 & 1493 & 8,969 & 0,000 & **** \\
\hline
\end{tabular}




\section{Anhang C: Ein Simulationsexperiment mit GTAP}

\section{GTAP .cmf Datei zur Replikation des Simulationsexperiments in Kapitel 4.2.1}

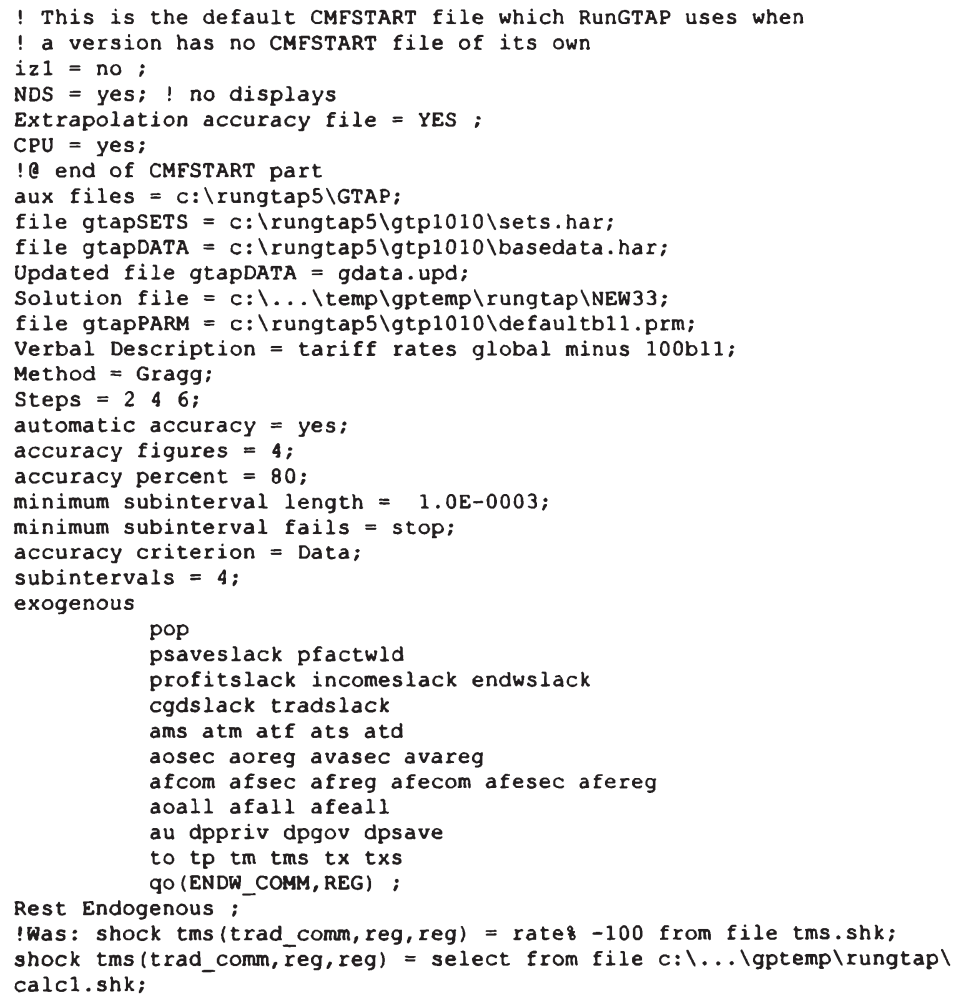




\section{Datei zur Aggregation der GTAP 6.01 Datenbasis für das Simulationsexpe- riment in Kapitel 4.2.1 (in zwei Spalten je Seite, Leertasten entfernt):}

Spalte 1:

! This file was created by program GtapAgg.

! If you edit it directly, please observe the following rules:

! The file has 6 sections:

! A section marker is a line that starts with "=".

! Each section begins AND ends with its own section markers.

! Hence the file must contain 12 section marker lines.

! Blank lines are ignored.

! Lines starting with "!" are ignored.

! Below, "sector" refers to "tradeable

commodity"

! and "factor" refers to "endowment

commodity".

!Section 1 lists new (aggregated) sectors.

!Each line consists of:

! (a) name of new sector (max 12 letters)

! (b) an ampersand "\&"

! optionally, (c) description of new

sector (max 30 letters)

!You may have to add or delete lines in

Section 1

!Section 2 lists original (disaggregated) sectors.

!Each line consists of:

! (a) name of old sector (max 12 letters) ! optionally, (b) space or comma followed by description of new

! sector ( $\max 30$ letters)

! (c) an ampersand "\&"

! (d) matching new sector (must be one of the short

! names mentioned in Section 1

!You are not expected to add or delete

lines in Section 2,

lor to alter the stuff BEFORE the "\&" on each line

! Sections 3 and 4 specify the regional aggregation.

! They follow the same pattern as

sections 1 and 2 .

! Sections 5 and 6 specify the factor

(endowment) aggregation.

! They follow a similar pattern to

Sections 1 and 2 .

! However see notes preceding section 5 .

\section{Spalte 2:}

! Section 1

! One line for each new sector

$=====$

pdr \& Paddy rice

pcr \& Processed rice

wheat \& Wheat

animal \& Animal products

meat \& Meat products

milkdairy \& Milk and dairy products

othcrop \& Other crops

othproc \& Other processed products

Mnfes \& Manufactures

Svces \& Services

$====$

! Section 2

! One line for each old sector

! Old sector at left, corresponding new sector on right

$=====$

pdr Paddy rice \& pdr

wht Wheat \& wheat

gro Cereal grains nec \& othcrop

v_f Vegetables, fruit, nuts \& othcrop

osd Oil seeds \& othcrop

c b Sugar cane, sugar beet \& othcrop

pfb Plant-based fibers \& othcrop

ocr Crops nec \& othcrop

ctl Cattle, sheep, goats, horses \& animal

oap Animal products nec \& animal

rmk Raw milk \& milkdairy

wol Wool, silk-worm cocoons \& animal

frs Forestry \& Mnfcs

fsh Fishing Mnfes

coa Coal Mnfcs

oil Oil \& Mnfes

gas Gas Mnfes

omn Minerals nec \& Mnfcs

cmt Meat: cattle, sheep, goats, horse \&

meat

omt Meat products nec \& meat

vol Vegetable oils and fats \& othproc

mil Dairy products \& milkdairy

pcr Processed rice \& pcr

sgr Sugar \& othproc

ofd Food products nec \& othproc

b t Beverages and tobacco products \& othproc

tex Textiles \& Mnfcs

wap Wearing apparel \& Mnfcs

lea Leather products \& Mnfcs

lum Wood products \& Mnfes

ppp Paper products, publishing \& Mnfcs

p_c Petroleum, coal products \& Mnfcs

crp Chemical, rubber,plastic prods \& 


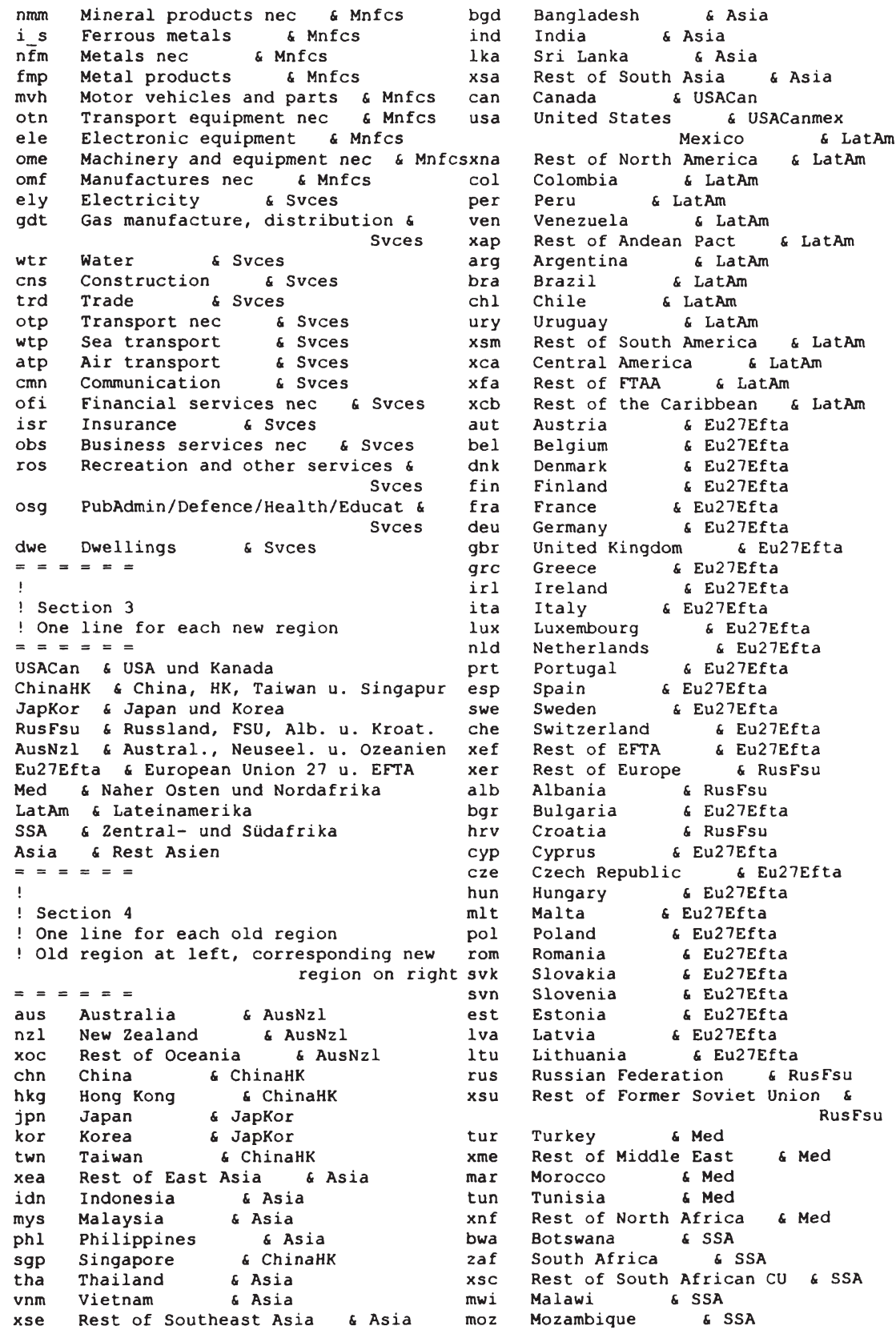




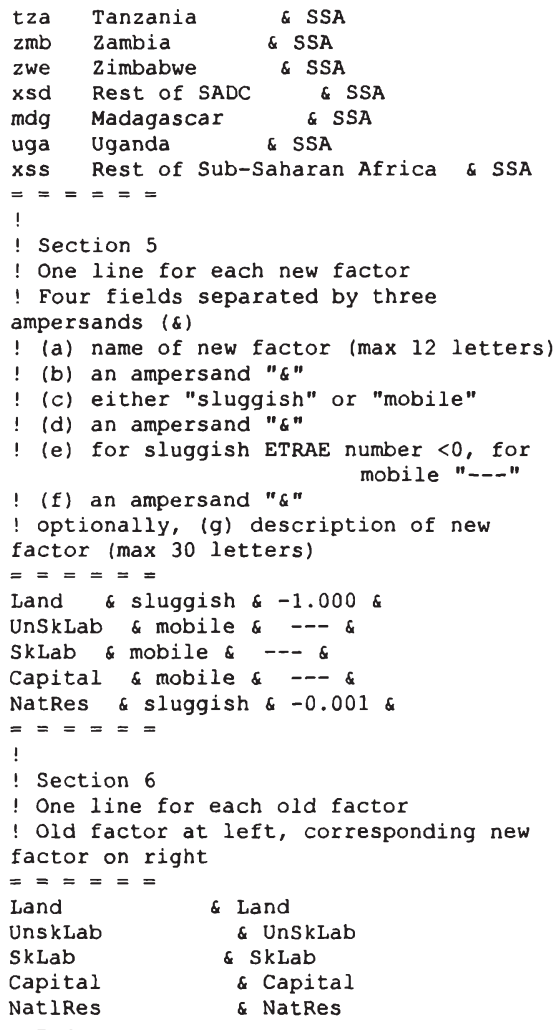


cege-Schriften

Das cege - Centrum für Europa-, Governance- und Entwicklungsforschung - wurde 1999 von der Wirtschaftswissenschaftlichen Fakultät der Georg-August-Universităt gegrūndet. Das cege dient als Forum zur internationalen und interdisziplinären Forschungszusammenarbeit in den Themenbereichen Europăische Integration, Govemance und Entwicklungsökonomik. In den cege-Schriften werden Forschungsergebnisse aus Dissertationen, Habilitationen oder anderen Forschungsprojekten des cege veróffentlicht.

Band 1 Axel Gerloff: Wechselkurspolitik in Mittel- und Osteuropa. Eine theoretische Analyse unter besonderer Berūcksichtigung der Erfahrungen der zehn Kandidaten für eine EU-Osterweiterung. 2001.

Band 2 Ingo Konrad: Zur Integration ausgewählter mittel- und osteuropäischer Lănder in die währungspolitische Ordnung Europas. 2002.

Band 3 Axel Hennighausen: Wegekostenfinanzierung und Lenkung im deregulierten europăischen Verkehrsmarkt. 2002.

Band 4 Wolfgang Münch: Effects of EU Enlargement to the Central European Countries on Agricultural Markets. 2002.

Band 5 Holger Niermann: Welchen Finanzausgleich braucht Europa? Ein konkreter Vorschlag zur Neugestaltung der europăischen Finanzbeziehungen mit Simulationsmodell. 2002.

Band 6 Jens Südekum: Agglomeration and Regional Unemployment Disparities. A Theoretical Analysis with Reference to the European Union. 2003.

Band 7 Christoph Schinke: Der Geldmarkt im Euro-Wāhrungsraum. Geldmarktgeschäfte, Zinsbildung und die Taylor Rule. 2004.

Band 8 Kai Stukenbrock: The Stability of Currency Boards. 2004.

Band 9 Harald Grethe: Effects of Including Agricultural Products in the Customs Union between Turkey and the EU. A Partial Equilibrium Analysis for Turkey. 2004.

Band 10 Götz Zeddies: Perspektiven der Gemeinschaftspolitiken in der erweiterten EU. 2005.

Band 11 Ingmar Kumpmann: Systemwettbewerb und Umverteilung. Gefährdet die Globalisierung den Sozialstaat? 2005.

Band 12 Katarzyna Haverkamp: Bestimmungsfaktoren der Vertragsforschung in Deutschland. Eine theoretische und okonometrische Analyse. 2007.

Band 13 Peter Schwarz: Kapitalsteuerwettbewerb zwischen Nationalstaaten unter besonderer Berūcksichtigung Deutschlands. Eine empirische Analyse. 2007.

Band 14 Andreas Poppe: Auswirkungen der Einführung einer konsolidierten Körperschaftsteuer-Bemessungsgrundlage in der Europäischen Union. Eine empirische Analyse der Konzernstrukturen und des Steueraufkommens. 2008.

Band 15 Sebastian Hess: Meta-Analyse angewandter Gleichgewichtsmodelle des internationalen Agrarhandels. 2008.

www.peterlang.de 
Sebastian Hess - 978-3-631-75694-2

Downloaded from PubFactory at 01/11/2019 02:52:17AM

via free access 


\section{Perspektiven der \\ Gemeinschaftspolitiken in der erweiterten EU}

Frankfurt am Main, Berlin, Bern, Bruxelles, New York, Oxford, Wien, 2005. 380 S., zahlr. Abb. und Tab.

CeGe-Schriften. Verantwortliche Herausgeberin: Renate Ohr. Bd. 10 ISBN 978-3-631-53399-4 • br. € 56.50*

In der Arbeit wird die Frage analysiert, welche Konsequenzen sich aus der Osterweiterung für die zukünftige Ausgestaltung der EU-Politiken ergeben können. Die mit der Erweiterung einhergehende zunehmende Heterogenität der ökonomischen Strukturen innerhalb der Europäischen Union lässt auf eine gleichermaßen zunehmende Pluralität der politischen Interessen der Mitgliedstaaten schließen, so dass in der erweiterten Union unter Umständen andere Interessen dominieren und andere einflussreiche Ländergruppen existieren können als dies in der alten EU der Fall war. Für ausgewählte Politikbereiche zeigt die empirische Analyse, dass sich in der erweiterten Europäischen Union verschiedene Ländergruppen mit relativ heterogenen politischen interessen gegenüberstehen, wodurch der politische Entscheidungsprozess auf europäischer Ebene durch die Erweiterung aller Voraussicht nach deutlich erschwert werden wird.

Aus dem Inhalt. Der europäische Entscheidungsprozess aus polit-ökonomischer Perspektive - Der europäische Entscheidungsprozess aus politischer und institutioneller Perspektive - Ökonomische Aspekte der Osterweiterung · Prognose der zukünftigen Entwicklung ausgewählter EU-Politiken

Frankfurt am Main - Berlin - Bern - Bruxelles - New York - Oxford - Wien

Auslieferung: Verlag Peter Lang AG

Moosstr. 1, CH-2542 Pieterlen

Telefax 0041 (0) $32 / 3761727$

*inklusive der in Deutschland gültigen Mehrwertsteuer

Preisänderungen vorbehalten

Homepage http://www.peterlang.de 
Sebastian Hess - 978-3-631-75694-2

Downloaded from PubFactory at 01/11/2019 02:52:17AM

via free access 